Development of Wastewater Pipe Performance Index and Performance Prediction Model

Thiti Angkasuwansiri

Dissertation submitted to the Faculty of the Virginia Polytechnic Institute and State University in partial fulfillment of the requirements for the degree of

Doctor of Philosophy

In

Civil Engineering

Sunil K. Sinha, Chair

Gerardo W. Flintsch

Russell A. Green

Michael R. Taaffe

April 26 ${ }^{\text {th }}, 2013$

Blacksburg, Virginia

Keywords: Wastewater Pipe Infrastructure, Standard Data Structure, Pipe Failure Mode and Mechanism, Performance Index, Performance Prediction 


\title{
Development of Wastewater Pipe Performance Index and Performance Prediction Model
}

\author{
Thiti Angkasuwansiri
}

\begin{abstract}
Water plays a critical role in every aspect of civilization: agriculture, industry, economy, environment, recreation, transportation, culture, and health. Much of America's drinking water and wastewater infrastructure; however, is old and deteriorating. A crisis looms as demands on these systems increase. The costs associated with renewal of these aging systems are staggering. There is a critical disconnect between the methodological remedies for infrastructure renewal problems and the current sequential or isolated manner of renewal analysis and execution. This points to the need for a holistic systems perspective to address the renewal problem. Therefore, new tools are needed to provide support for wastewater infrastructure decisions. Such decisions are necessary to sustain economic growth, environmental quality, and improved societal benefits. Accurate prediction of wastewater pipe structural and functional deterioration plays an essential role in asset management and capital improvement planning. The key to implementing an asset management strategy is a comprehensive understanding of asset condition, performance, and risk profile.
\end{abstract}

The primary objective of this research is therefore to develop protocols and methods for evaluating the wastewater pipe performance. This research presents the life cycle of wastewater pipeline identifying the causes of pipe failure in different phases including design, manufacture, construction, operation and maintenance, and repair/rehabilitation/replacement. Various modes and mechanisms of pipe failure in wastewater pipes were identified for different pipe material which completed with results from extensive literature reviews, and interviews with utilities and pipe associations. After reviewing all relevant reports and utility databases, a set of standard pipe parameter list (data structure) and a pipe data collection methodology were developed. These parameters includes physical/structural, operational/functional, environmental and other parameters, for not only the pipe, but also the entire pipe system. This research presents a development of a performance index for wastewater pipes. The performance index evaluates each 
parameter and combines them mathematically through a weighted summation and a fuzzy inference system that reflects the importance of the various factors. The performance index were evaluated based on artificial data and field data to ensure that the index could be implemented to real scenarios. Developing a performance index led to the development of a probabilistic performance prediction model for wastewater pipes. A framework would enable effective and systematic wastewater pipe performance evaluation and prediction in asset management programs. 


\section{Acknowledgement}

I would like to thank many people who have helped me through the completion of this dissertation. First of all, I would like to thank my advisor, Dr. Sunil Sinha for his guidance, encouragement, and patience throughout my Ph.D. study. His vision and motivation deeply inspired me. It was a great privilege to work and study under his guidance. Special thanks to my committee members, Dr. Gerardo Flintsch, Dr. Russell Green, and Dr. Michael Taaffe, for their helpful suggestions and critical comments on this research. I would like to take this opportunity to thank Water Environmental Research Foundation (WERF) that has funded my research project. I am always grateful for all of my friends at Virginia Tech, particularly Shaoqing Ge, Leon Gay for their help and suggestions. I would like to express my gratitude to my family, parents and brother, for their love and continuing support. I would not have completed this research work without them. Finally, I would like to thank my wife Arpajit Arnontavilas who is always be there for me throughout the years. Her love and caring mean everything to me. 


\section{Table of Contents}

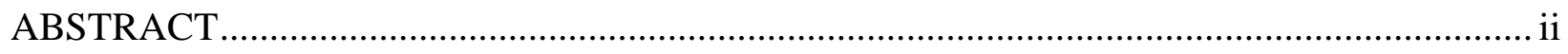

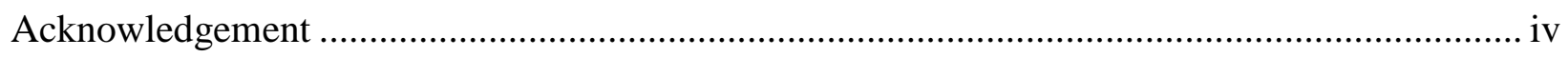

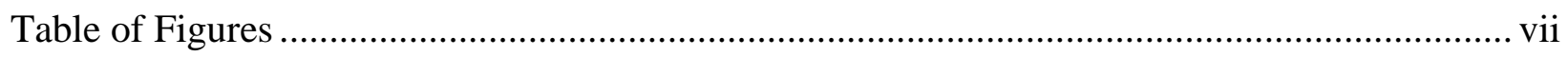

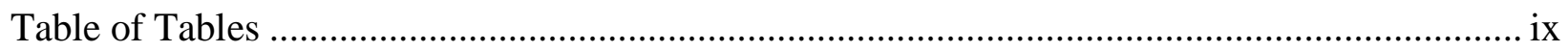

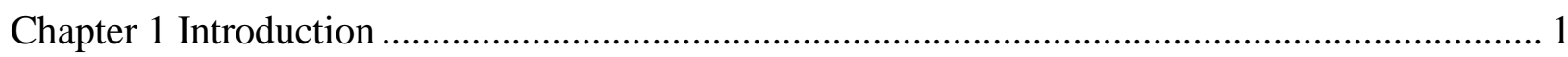

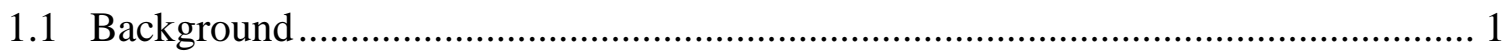

1.2 Motivation for the Research and Problem Statement ............................................ 2

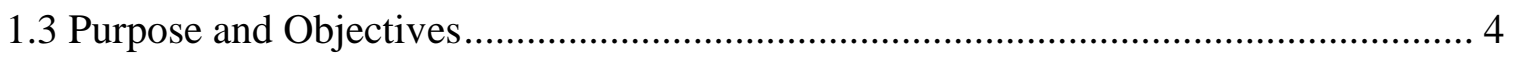

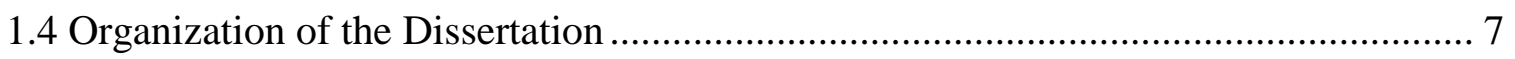

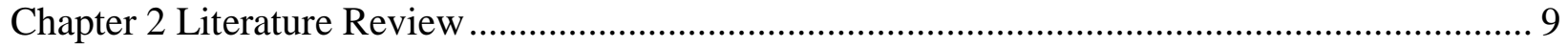

2.1 Wastewater Infrastructure Asset Management ................................................. 9

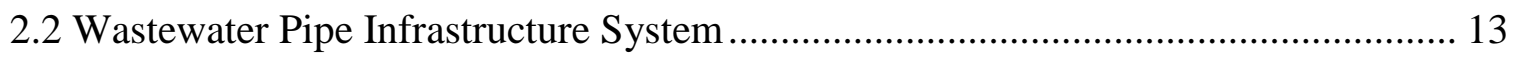

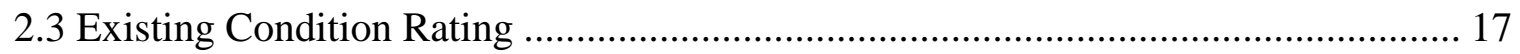

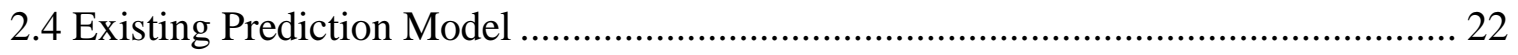

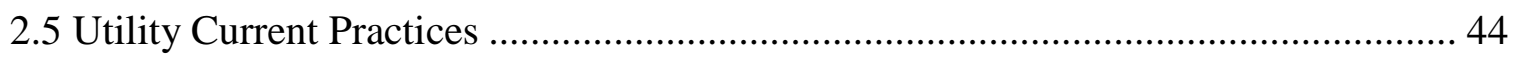

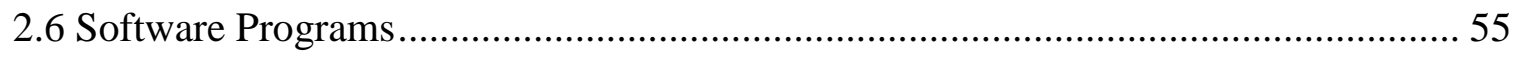

Chapter 3 Wastewater Pipe Failure Modes and Mechanisms ................................................ 57

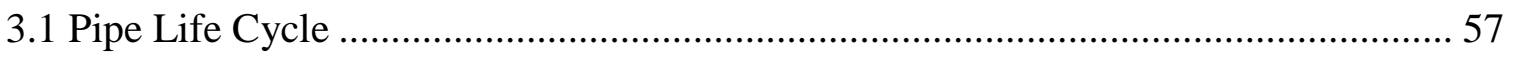

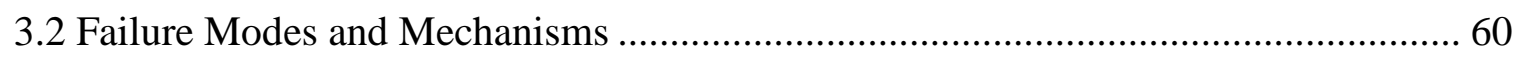

Chapter 4 Development of a Wastewater Pipe Performance Index........................................ 66

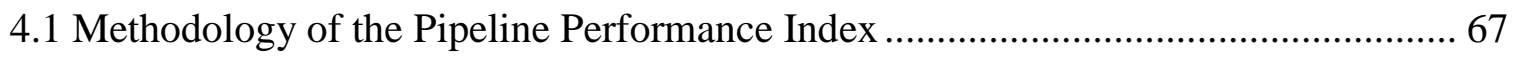

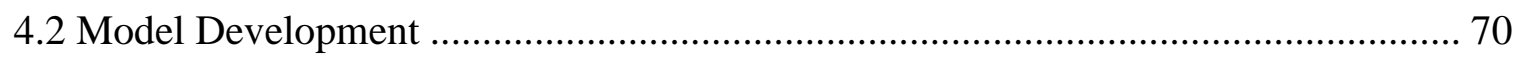

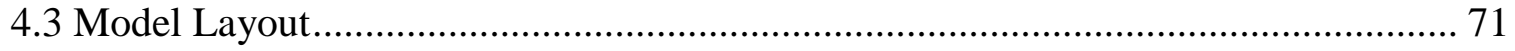

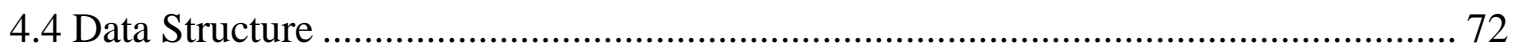

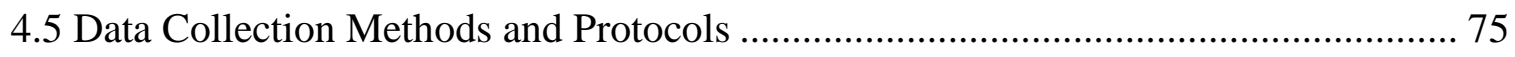

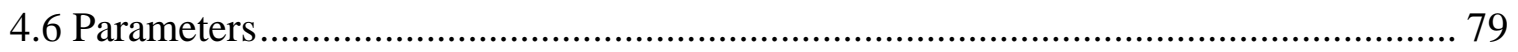




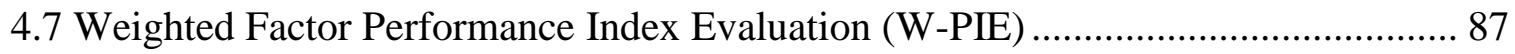

4.8 Fuzzy Inference Performance Index Evaluation Model (F-PIE) ............................ 95

Chapter 5 Wastewater Pipe Performance Index Evaluation ................................................ 102

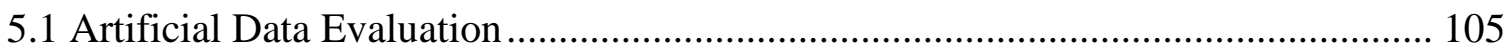

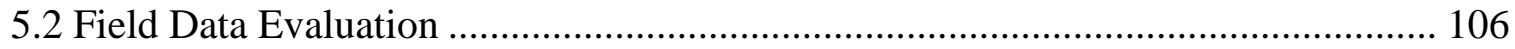

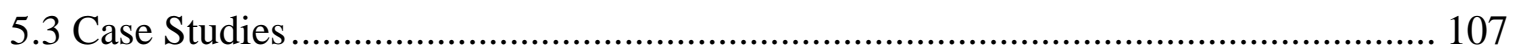

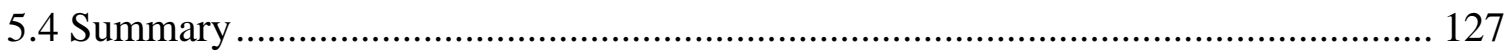

Chapter 6 Development of a Framework for Wastewater Pipe Performance Prediction ........... 128

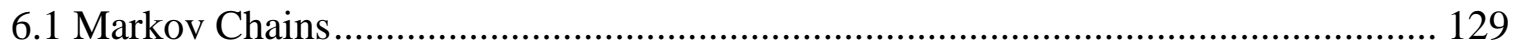

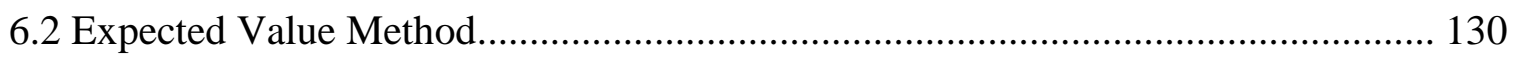

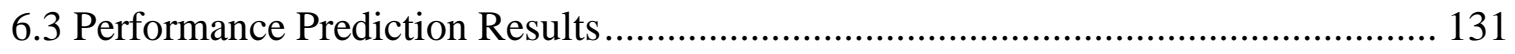

Chapter 7 Conclusions and Recommendations................................................................ 138

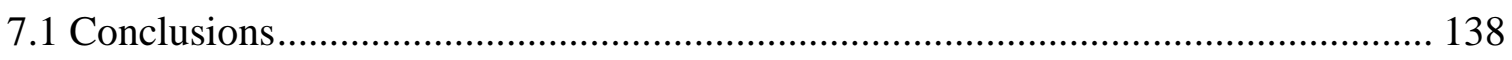

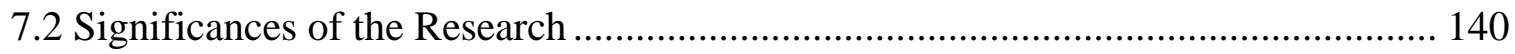

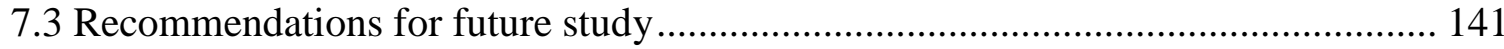

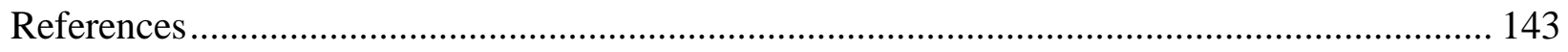

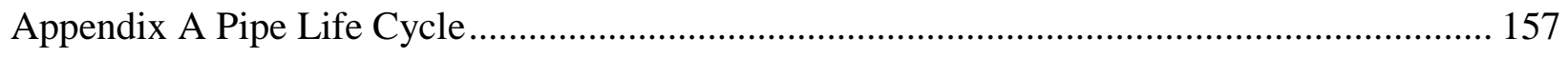

Appendix B Failure Mode and Mechanism .............................................................. 170

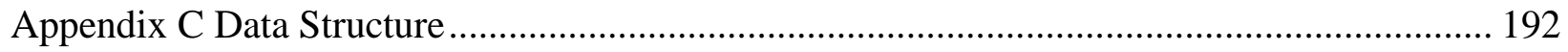

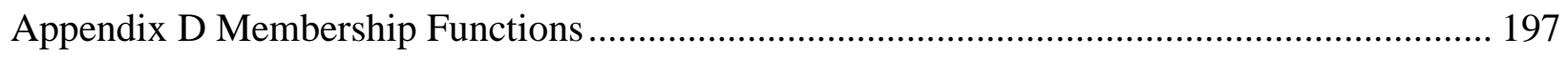

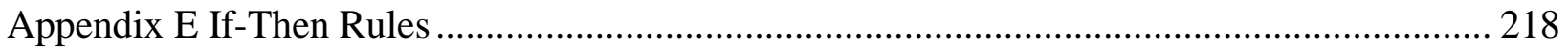

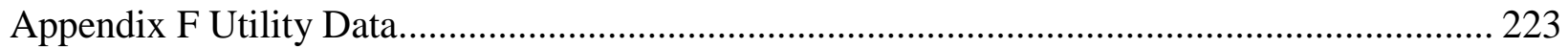

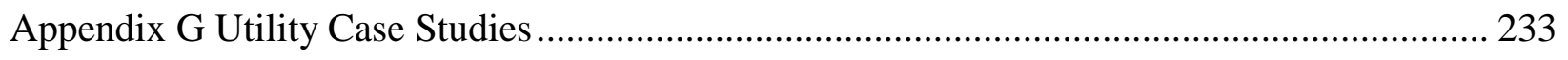

Appendix H Examples of Questionnaire Responses ....................................................... 279 


\section{Table of Figures}

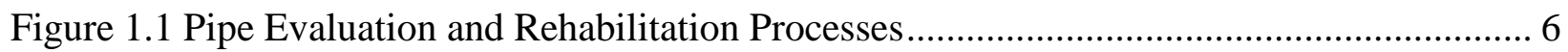

Figure 1.2 Research Approach.......................................................................................... 7

Figure 2.1 Water (Drinking Water and Wastewater) Infrastructure System ............................... 10

Figure 2.2 Factors Affecting the Condition and Performance of Buried Pipes ............................ 14

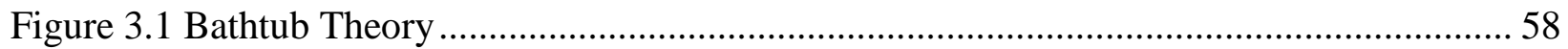

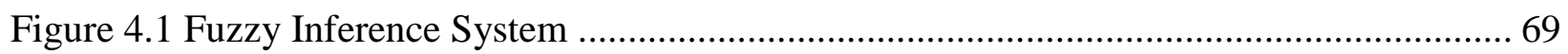

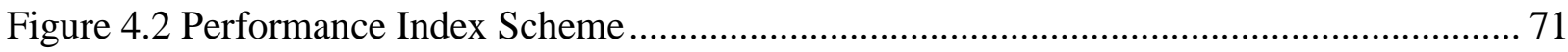

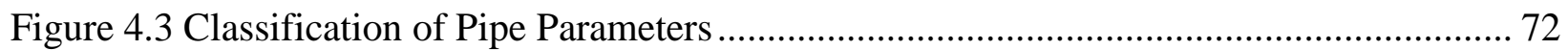

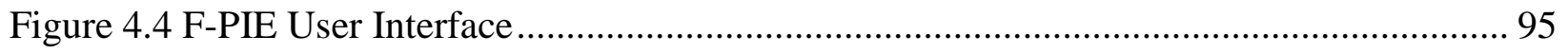

Figure 4.5 Example of Membership Functions......................................................................... 96

Figure 5.1 Evaluation Methodology Framework ………….................................................... 105

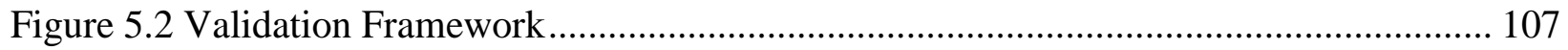

Figure 5.3 Target Utilities for Pilot Study of the Model............................................................ 108

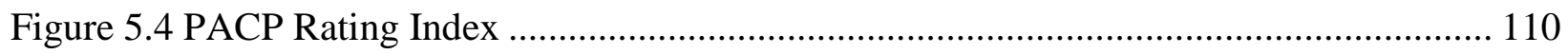

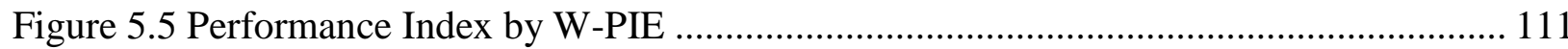

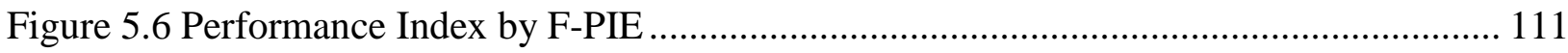

Figure 5.7 Performance Index by No PACP Data, PACP, F-PIE, and W-PIE............................ 112

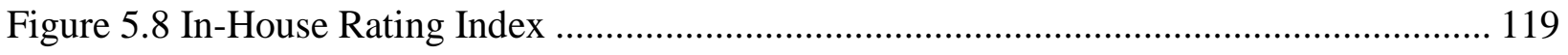

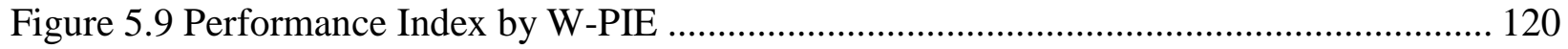

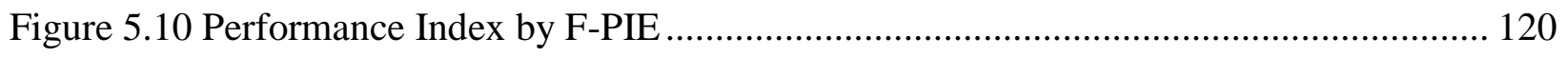

Figure 5.11 Performance Index by In-House Rating System, W-PIE and F-PIE....................... 121

Figure 5.12 WSSC's L2 Score vs. Fuzzy Inference Performance Index .................................. 125 
Figure 5.13 WSSC's Overall Condition Score vs. Fuzzy Inference Performance Index

Figure 5.14 WSSC's L1 Score vs. Fuzzy Inference Performance Index (without inspection data)

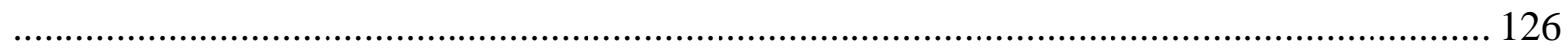

Figure 6.1 Performance Prediction for Concrete Pipe …………………………………........... 134

Figure 6.2 Performance Prediction for Vitrified Pipe................................................................. 136

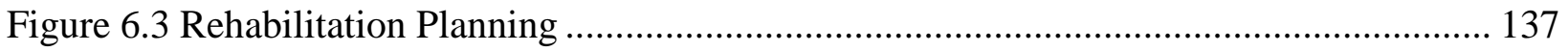




\section{Table of Tables}

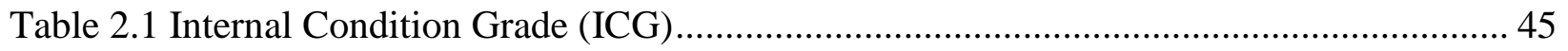

Table 2.2 Condition Assessments and Planning Technique ……………………...................... 56

Table 3.1 Concrete Wastewater Pipe Life Cycle ....................................................................... 59

Table 3.2 Failure Modes of Concrete Wastewater Pipe .............................................................. 61

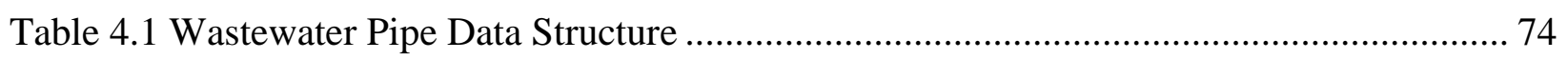

Table 4.1 Wastewater Pipe Data Structure cont ……………....................................................... 75

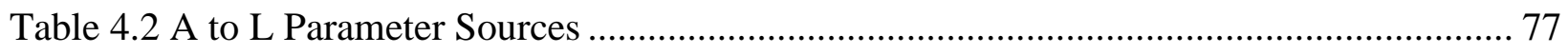

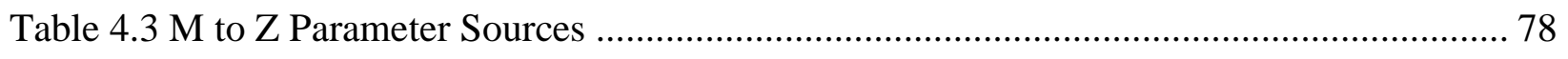

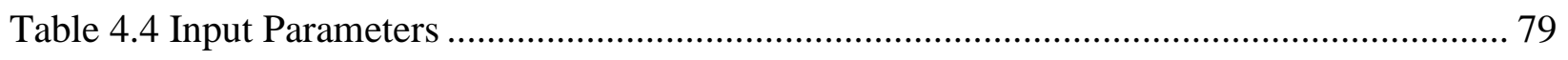

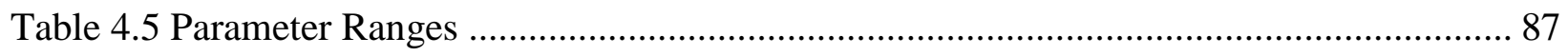

Table 4.6 Parameter Weight by Module (Structural Index) ……………………...................... 92

Table 4.7 Parameter Weight by Module (Functional Index) ........................................................ 94

Table 4.8 Fuzzy Inference Model Membership Ranges (Integrity) ............................................... 97

Table 4.9 Fuzzy Inference Model Membership Ranges (Internal Corrosion) .............................. 98

Table 4.10 Fuzzy Inference Model Membership Ranges (External Corrosion) ............................ 98

Table 4.11 Fuzzy Inference Model Membership Ranges (Surface Wear).................................... 99

Table 4.12 Fuzzy Inference Model Membership Ranges (Blockage) .......................................... 99

Table 4.13 Fuzzy Inference Model Membership Ranges (In-exfiltration) ................................. 100

Table 4.14 Fuzzy Inference Model Membership Ranges (Root Intrusion) ................................ 100

Table 4-15 Fuzzy Inference Model Membership Ranges (Capacity) ……………...................... 100

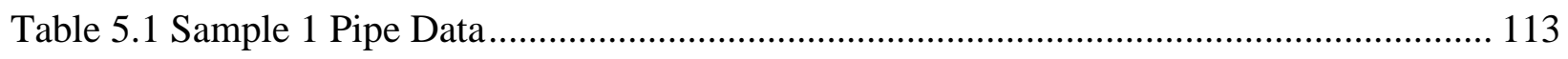

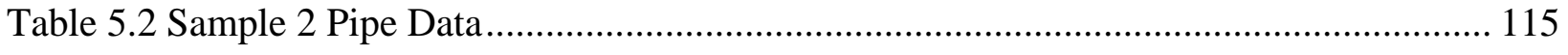




\section{CHAPTER 1}

\section{INTRODUCTION}

This chapter begins with a brief background presenting the introduction to wastewater pipe infrastructure. Afterwards, the motivation and problem statement are presented. Finally, the research purpose and objectives are introduced.

\subsection{Background}

The water infrastructure (drinking water and wastewater) has been built to provide the public with access to potable water and sanitation. The wastewater infrastructure system is a part of the water infrastructure. It is comprised of collection and transmission systems, wastewater treatment and sludge management systems, and reuse/ recycling systems. It collects, transmits, treats, and disposes of water supplies used by domestic, industrial, commercial, and public users (Grigg, 2002).

Most cities and towns started building collection systems over 100 years ago. Many of these systems have not received adequate upgrades, maintenance, repair, and rehabilitation over time (U.S.EPA, 2005). Today, municipal governments are facing an infrastructure crisis requiring costly renewal beyond their capacity. There has been a steady decline in the state of water infrastructure over the past two decades, and a growing concern is that these facilities may be 
inadequate both for current requirements and projected future growth (U.S.EPA, 2005). Funding for these needs is limited, and a deferred maintenance, out-of-sight, out-of-mind philosophy still prevails in many regions. Recently, the American Society of Civil Engineering (ASCE) in its 2013 assessment of the nation's infrastructure assigned the "D+" grade to the overall infrastructure and " $\mathrm{D}$ " to the wastewater infrastructure, and estimated the twenty-year investment needs to be in excess of \$298 billion for wastewater and storm water systems (ASCE, 2013). Although the federal government has spent more than $\$ 71$ billion on wastewater treatment programs since 1973, the nation's 19,000 wastewater systems still face enormous infrastructure funding needs in the next 20 years to replace pipes and other constructed facilities that have exceeded their design life (WIN, 2000). With billions of dollars being spent yearly for wastewater infrastructure, the systems face a shortfall of at least $\$ 21$ billion annually to replace aging facilities and comply with existing and future federal regulations (ASCE, 2000). Monetary investment alone will not resolve this dilemma; it must be met with a new approach to sustainable wastewater infrastructure engineering and management. "New solutions are needed to what amounts to nearly a trillion dollars in critical water and wastewater investments over the next two decades. Not meeting the investment needs of the next 20 years risks reversing the public health, environmental, and economic gains of the last three decades." (ASCE, 2000).

\subsection{Motivation for the Research and Problem Statement}

A critical disconnect exists between the methodological remedies for infrastructure renewal problems and the current sequential or isolated manner of renewal analysis and execution. The disconnect manifests in the need for a holistic systems perspective. New tools are needed to provide the intellectual support for utility assets decisions necessary to sustain economic growth, environmental quality, and improved societal health. Developing an efficient infrastructure management system can provide fast and reliable decision-making tools needed to handle the large volume of deteriorating buried pipeline infrastructure systems, particularly wastewater pipelines, which pose serious threats to the environment if they fail. A comprehensive understanding of asset condition and performance is the key to implementing infrastructure asset management strategies. Wastewater utilities need protocols and methods for predicting the performance of utility assets. Prediction modeling can be used to provide a time stream of benefits, costs, and level of service for both a single asset and a group of assets. 
Condition/Performance assessment and deterioration modeling are rapidly becoming an increasing part of life cycle asset management activities in the United States. In this research, a "total-systems" approach have been taken for development of protocols and methods for predicting the condition/performance deterioration of wastewater pipe infrastructure asset. The research focuses on collecting all relevant data, tools and methodologies to evaluate and predict the wastewater pipe performance in an overall utility asset management program. The research emphasizes on conducting analysis, assessment, and integration of data/information with existing methodologies for development of standard condition rating scale, developing robust probabilistic deterioration models for predicting the performance of wastewater pipe assets; it will evaluate this approach in a real-world pilot project. The research program is envisioned as a model system that can be implemented at municipalities and utilities around the country. The overall objective of this research is to provide utilities with tools and methodologies to achieve long-term asset management programs and integrate with an overall asset management program.

The performance evaluation and framework can be enhanced by addressing the issues outlined below:

- Lack of a clear understanding of pipe failure modes and mechanisms. Pipe failure mode and mechanism differ significantly by pipe material. Although, there are many researches on this topic, a comprehensive list of failure modes and mechanisms of wastewater pipes by pipe material does not exist. A complete understanding of all failure modes and mechanisms is needed in order to properly assess the current condition of the pipe.

- Lack of a standard data structure. Data is needed in assessing a pipe condition and performance. Some parameters are more significant than others so a welldefined data structure would enable the utility to appropriately collect data. A close examination and careful identification of performance parameters will help developing an accurate prediction model (Park, 2009)

- Lack of a performance evaluation tool which takes into consideration different factors such as pipe characteristics, internal and external environmental factors. Currently, many utilities assess the wastewater pipe condition and performance in their own manner based on their experience. This method may provide correct 
results; however, a more standard tool should be developed to ensure the condition assessment process (Mehta, 2006).

- Lack of a performance prediction tool. Prediction tool is a significant input for pipe renewal programs. Effective prediction model should be developed and calibrated based on a well-created condition/performance evaluation system and a complete pipe data required. One of the biggest challenges in the preparation of accurate prediction model is to develop a standardized condition rating system (Baik, 2006).

\subsection{Purpose and Objectives}

The main purpose of this research is to develop protocols and methods for predicting the condition/performance deterioration of wastewater pipe system. A total-system approach is taken to achieve the purpose of this research which 3 objectives needed to be fulfilled as follows;

1. Developing a data structure for wastewater pipe system to support the performance index and prediction model with the understanding of failure modes and mechanisms of wastewater pipe based on material type

2. Developing robust condition and performance indexes for wastewater pipe system

3. Developing prediction model for wastewater pipe system

\section{Objective 1 Data Structure}

There are many parameters affecting wastewater pipe system and their failure. Examples of these parameters are structural parameters - pipe diameter, age, and material, and environmental parameters - soil properties and external loading. In this research, parameters and their effects on wastewater pipe system will be investigated. To develop a robust performance index and performance prediction model, we need to understand pipe failure modes and mechanisms based on pipe life cycle and identify parameters that affect pipe failure process. Pipeline in municipal wastewater systems is comprised of different types of material depending on suitability of the pipe location and design purposes. Each pipe material undergoes failure in a different way. Studies have shown that the structural performance and behavior of the buried pipe depend on the type of backfill placed around the pipe, construction sequence, compaction control, surface loads, and the 
type of pipe material (flexible or rigid) (Makar, 2000; Rajani, 1996; Boot, 1998; Heger, 1985; Davies, 1999; Serpente, 1993).

Rigid pipe is designed to resist external loads by its inherent strength, whereas flexible pipe relies on the capacity of the surrounding soil to carry the load and provide stability. All types of pipe can perform well, but the conditions for satisfactory long-term performance vary. Furthermore, the performance criteria are different for the different types of pipe: the severity of cracking is the main performance criterion for rigid concrete pipe; whereas the degree of deflection is the main performance criterion for flexible pipe. For the purpose of this study, it is necessary to develop a complete understanding of the failure modes and mechanisms of wastewater pipe system.

\section{Objective 2 Performance Index}

At present, utilities rely mostly on CCTV (Closed-circuit television) inspection in evaluating pipes and making rehabilitation decisions. Images from CCTV inspection are rated using in-house or National Association of Sewer Service Companies (NASSCO)'s rating systems. However, the CCTV does not capture all data needed in predicting wastewater pipe failures. This research proposes the methods to evaluate the pipe condition and performance through other important data such as structural, environmental, operational, and other data in addition to CCTV data. With this combination of data, the better condition and performance index can be developed. The figure 1.1 shows the current practice that many utilities employ comparing to the methodology. 


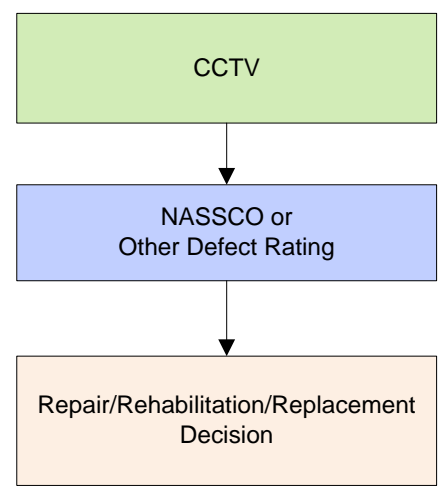

Current Practice

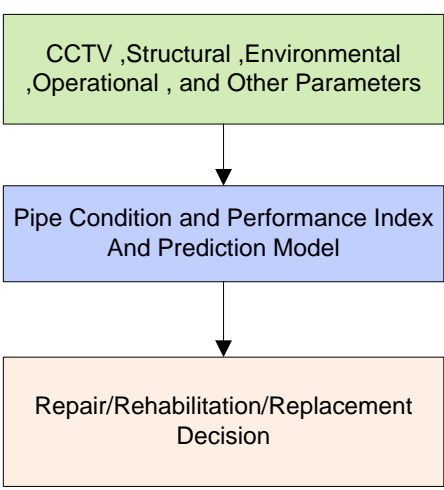

Proposed Methodology

\section{Figure 1.1 Pipe Evaluation and Rehabilitation Processes}

\section{Objective 3 Prediction Model}

A pipe prediction model (deterioration model) represents the condition of a pipe over time. This pipe prediction model helps the utility in estimating the condition or remaining service life of a particular pipe section, and identifying the high risk area where the repair, replacement, or rehabilitation is needed plus the model aids decision-making in capital improvement program (CIP). Deterioration models can be grouped into the following main categories;

Deterministic Model Approach: The deterministic models can predict the performance of buried pipes. Deterministic models can be classified into the following two classes:
a. Mechanistic models
b. Empirical models

Some of the existing models in the transportation industry have been developed through regression analysis and combined mechanistic-empirical analysis. Data collection and lab/field experimental tests are required to develop these models.

Statistical Model Approach: The statistical models can predict a distribution of value for dependent variables, such as the condition of buried pipes. Historical data-based models can be applied to forecast future failure rates of infrastructure. For example, an exponential function could represent the increase in water main failure rates with pipe age (Shamir, 1979). Deterioration models for 
pipe infrastructure were developed using the Markov chains approach and data from sewer pipe inspection records (Kathula, 2001; Wirahadikusumah, 2001; Abraham, 1998).

Heuristic Model Approach: The heuristic models commonly are applied for large and not wellunderstood infrastructure problems producing sub-optimal solutions. These models can be developed through subjective opinion from field engineers and experts. Artificial Neural Networks (ANN) and Fuzzy Logic approach have been used for infrastructure deterioration. Tran et al. (Tran, 2007) developed an ANN to predict an average failure rate in cast iron pipes. Kleiner et al. (Kleiner, 2005) used Fuzzy Logic to model deterioration and failure in PCCP and Cast/Ductile Iron pipes.

With the right parameter information and historical performance data, an analysis to predict and model deterioration of the pipe can be done. The overall research approach is illustrated in figure 1.2 .

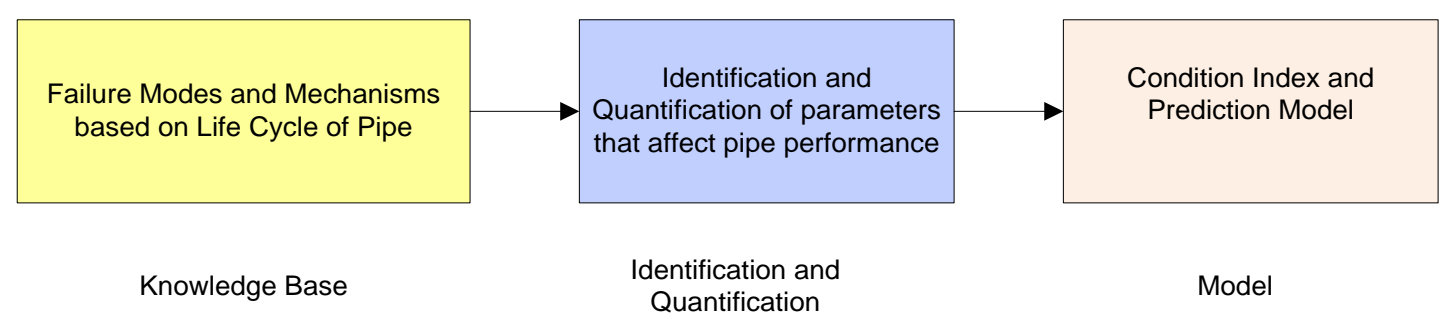

Figure 1.2 Research Approach

\subsection{Organization of the Dissertation}

The dissertation is organized in the following order;

Chapter 1: Introduction - This chapter begins with a brief background presenting the introduction to wastewater pipe infrastructure. Afterwards, the motivation and problem statement are presented. Finally, the research purpose and objectives are introduced. 
Chapter 2: Literature Review - This Chapter presents the relevant literature review for the topics of this study. The first section provides background information on wastewater infrastructure asset management and pipe infrastructure systems. This is followed by state of the art review on condition/performance index and prediction model. Lastly, the utility current practices are discussed.

Chapter 3: Failure Modes and Mechanisms - This chapter provides a complete list of life cycle and failure modes and mechanisms of wastewater pipe based on material along with the data collection method and protocol.

Chapter 4: Development of a Wastewater Pipe Performance Index - This chapter presents the methodology for developing a wastewater pipe performance index, the weighted factor and fuzzy inference models. This chapter presents a comprehensive list wastewater pipe data structure detailing all parameters that affect wastewater pipe performance.

Chapter 5: Wastewater Pipe Performance Index Evaluation - This chapter provides evaluation of the developed performance index could be implemented to a real world database.

\section{Chapter 6: Development of a Framework for Wastewater Pipe Performance Prediction -} This chapter presents a framework for developing a wastewater pipe performance prediction model.

Chapter 7: Conclusions and Recommendations - The chapter presents findings and conclusions of the work completed in this research, and provide future recommendations. 


\section{CHAPTER 2}

\section{LITERATURE REVIEW}

This Chapter presents the relevant literature review for the topics of this study. The first section provides background information on wastewater infrastructure asset management and pipe infrastructure systems. This is followed by state of the art review on condition/performance index and prediction model. Lastly, the utility current practices are discussed.

\subsection{Wastewater Infrastructure Asset Management}

Water from sources like surface water or ground water are sources of input for a water treatment plant. This water is purified to potable water and then distributed for use in residential, commercial and industrial places. Later, the wastewater from the users is collected and transported to the wastewater treatment plant. Finally, the treated water is sent back to the nearby water bodies. This Municipal water cycle is illustrated in Figure 2.1. 


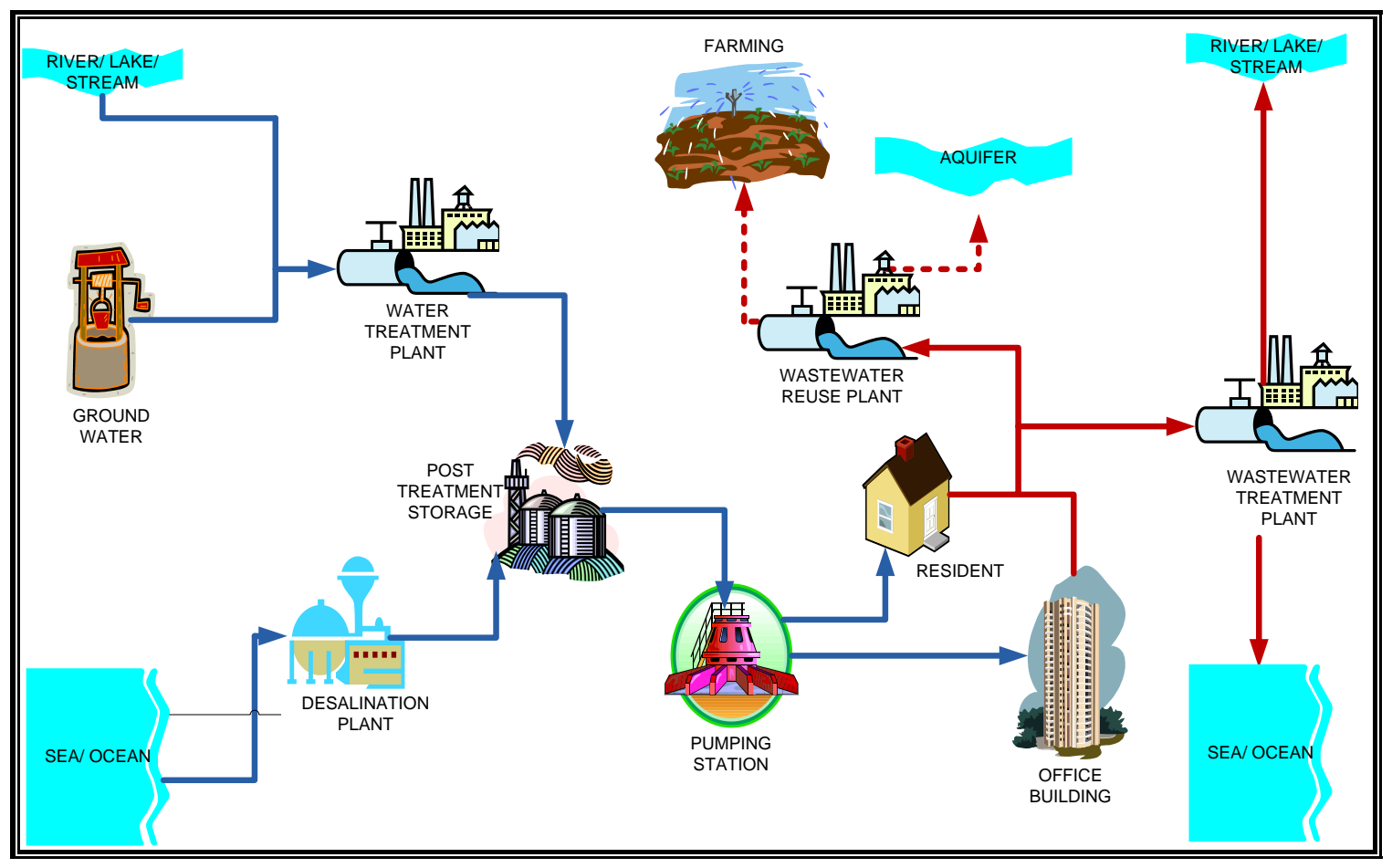

Figure 2.1 Water (Drinking Water and Wastewater) Infrastructure System

The water infrastructure (drinking water and wastewater) has been built to provide the public with access to potable water and sanitation.

The wastewater infrastructure system is a part of the water infrastructure. It is comprised of collection and transmission systems, wastewater treatment and sludge management systems, and reuse/ recycling systems. It collects, transmits, treats, and disposes of water supplies used by domestic, industrial, commercial, and public users (Grigg, 2002).

\section{Collection and Transmission Systems}

Wastewater collection and transmission systems are designed to collect and transport wastewater from domestic, commercial, and industrial sources to treatment plants for processing (Heastad, 2004). The process begins when service laterals or sewer pipes convey the wastewater away from the buildings where it connects to a main sewer pipe. The main sewer pipe then transports the wastewater to the treatment plant or a pump station where the wastewater flow is pumped into the wastewater treatment plant. Once treated, the water is discharged into a stream 
or a larger body of water. Collection systems in large communities may be comprised of pipes which convey the wastewater by means of pumps and gravity. The design of a gravity system is usually to have an operating velocity of at least $2 \mathrm{ft} / \mathrm{s}$ to preclude the deposition of solids. Some of the main considerations for the design of a wastewater pipe are the pipe-soil interaction, slope, materials resistance to scour, and effective joints which minimize or eliminate any infiltration of groundwater into the pipe or exfiltration of the wastewater to the surrounding soils and groundwater areas (Najafi, 2005).

Wastewater usually flows under gravity, however pressure flows do exist. Types of pressure flow are:

- Force Mains: sewage mainline is pumped where gravity flow is not possible

- Pressure Sewers: each household has a pump that discharges to the pressure main

- Vacuum Sewers: the vacuum pump pulls the flow through the system

The collection system of the wastewater pipelines consists of separated sewers and combined sewers, where combined systems collect storm water and sanitary sewer.

\section{Wastewater Treatment System}

Wastewater is treated in the treatment plant before being discharge to the environment so that the disposed water does not contaminate the environment and harm the public health. In general, the treatment system aims to remove floating material, biodegradable organics, and pathogenic organisms. Considering public health, toxic chemicals also have to be suspended. Wastewater treatment plants are classified as physical, chemical, or biological.

Typical operations and processes of each unit (U.S.EPA, 1998),

- Physical: screening, mixing, flocculation, sedimentation, flotation, filtration, gas transfer

- Chemical: precipitation, adsorption, disinfection

- Biological process: various biological processes include active sludge, trickling filter, stabilization pond 
Wastewater treatment removes the following major contaminants: suspended solids, biodegradable organics, volatile organics, pathogens, nutrients, refractory organics, heavy metals, and dissolved organic solids. Industrial wastes may contain additional elements such as acids, high temperatures, toxic chemicals, oils, and greases that must be removed in the pretreatment process before going into wastewater plant. Most wastewater treatment plants in the United States can treat less than 1 million gallons per day (mgd), and are considered to be small plants. Smaller wastewater systems, typically 0.01-0.25 mgd, contain package plants or on-site systems, mainly septic tanks. The most conventional types are extended aeration, contact stabilization, sequencing batch reactors, rotating biological contactor, and physical/chemical. On-site systems are also available for individual homes or cluster of homes (U.S.EPA, 1998).

\section{Sludge Management System}

Sludge is a semi-solid substance left from wastewater treatment process. It can be categorized into raw primary sludge (fecal material) and secondary sludge (living culture organisms that help remove contaminants from wastewater). Before going to a landfill, sludge needs additional processing as follows: pumping, grinding, degritting, thickening, stabilization, conditioning, disinfection, dewatering, drying, thermal reduction, and ultimate disposal (U.S.EPA, 1998).

\section{Reuse/Recycling System}

Water reuse, also called water recycling and water reclamation. Water is recycled and reused in the natural water cycle, though water recycling refers to projects that use technology to speed up the natural processes (U.S.EPA, 1992). Water reuse has almost become necessary for conserving and extending available water supplies. In addition, Water reuse offers communities with an alternate wastewater disposal method and provides pollution abatement by diverting effluent discharge away from sensitive surface waters (U.S.EPA, 1992). As long as it is sufficiently treated, the recycled water can satisfy most water demands. U.S. Environmental Protection Agency (USEPA) provides a suggested water recycled treatment and uses. Examples of the uses are crop irrigation, industrial cooling processes, toilet flushing, and car washing. However if the human exposure to recycled water is likely, further treatment may be required to prevent disease 
or other contaminants. (U.S.EPA, 1998). This research will focus only on wastewater pipe infrastructure system which is the main component of the collection and transmission systems. The wastewater pipe infrastructure system is described in the next subchapter.

\subsection{Wastewater Pipe Infrastructure System}

Pipelines are the main component in wastewater collection systems. Most buried pipeline networks have been installed using open trench construction methods (Schrock, 1991). This construction method typically consists of placing pipes on bedding material and backfilling the trench. Studies have shown that the structural performance and behavior of the buried pipe is dependent on the type of backfill placed around the pipe, construction sequence, compaction control, surface loads, and the type of pipe material (Makar, 2000; Rajani, 1996; Boot, 1998; Heger, 1985; Davies, 1999; Serpente, 1993). The pipelines used in municipal wastewater systems are made of different types of material depending on suitability of the pipe location and design purposes. Each pipe material undergoes failure in a different way. Rigid pipe is designed to resist external loads by its inherent strength, whereas flexible pipe relies on the capacity of the surrounding soil to carry the load and provide stability. All types of pipe can perform well, but the conditions for satisfactory long-term performance vary. Furthermore, the performance criteria are different for the different types of pipe: the severity of cracking is the main performance criterion for rigid concrete pipe, whereas the degree of deflection is the main performance criterion for flexible pipe. For the purpose of analysis or design or both, it is necessary to develop a complete understanding of the failure modes and mechanisms of buried pipe infrastructure systems.

There are many parameters affecting wastewater pipe infrastructure systems and their failure. Examples of these parameters are structural such as pipe diameter, age, and material, and environmental such as soil properties and external loading. Figure 2.2 shows examples of some of these parameters which affect the pipe deterioration in both short-term and long-term. Changes in one parameter will also affect the others in many ways. For example, in concrete pipe excessive loadings along with poor pipe bedding can cause pipe cracks and fractures. Cracks and fractures in the pipeline will cause infiltration and exfiltration to/from environment. 


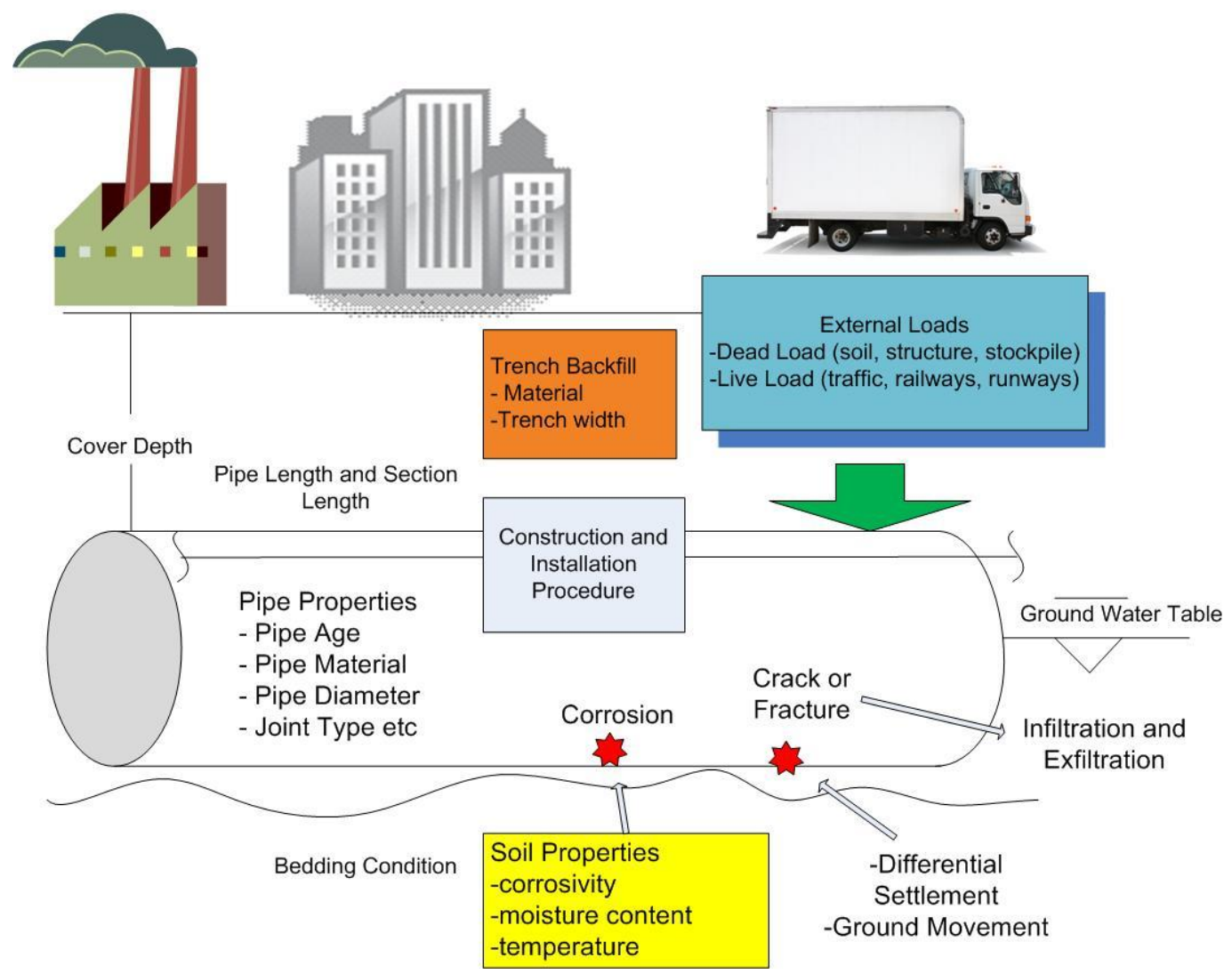

Figure 2.2 Factors Affecting the Condition and Performance of Buried Pipes

\section{Overview of U.S. Wastewater Collection and Transmission Systems}

The Clean Water Needs Survey done in 1998 (U.S.EPA, 2001) identified more than 19,500 municipal sewer collection systems nationwide, approximately 500,000 mi of municipally owned pipes in publicly owned systems and another 500,000 mi of privately owned pipes that transport wastewater into these collection systems.

The survey that was conducted on 42 wastewater utilities by American Society of Civil Engineers (ASCE) cooperating with U.S. Environmental Protection Agency (EPA) (Black \& Veatch, 1999) indicated the age of collection systems ranged from new to 117 years, with an average age of 33 years. About 18 percent of sewers were built in the last 10 years, 41 percent in the last 20 years, 82 percent in the last 50 years, and 98 percent in the last 100 years (Lai, 2008).

By evaluating population, urban density and public sewerage system data from the U.S. Census Bureau, the clean water and drinking water analysis (U.S.EPA, 2002) estimate that the average age of the sewer pipes is increase above 50 years by the year 2050. Believing that the 
installation of pipe has followed demographic increase in population and growth in metropolitan areas associated with suburbanization, the census expected an increase in population from 282 million in 2000 to more than 335 million by the year 2020. The system will need to keep up with the pace of population growth as well as mobilization of people.

Referring to a study performed by the Urban Institute (U.I., 1981), approximately 50 major main breaks and 500 stoppages occur per 1000 miles per year, amounting to an estimated 35,000 breaks and 350,000 stoppages annually (Tafuri, 2001). The problems caused by deterioration of jointing materials, pressure surges, disturbance by construction or direct tapping, and seismic activity conclude about 75 percent of the nation's piping systems functioning at 50 percent of capacity or less (Tafuri, 2001; ASCE, 1994).

\section{Wastewater Pipe Design and Installation}

Wastewater Pipes are made of various materials each of which has dissimilar advantages and disadvantages on certain aspects. Different pipe material has different design and installation standards. This subchapter gives an overview of design and installation procedures recommended by pipe associations and research studies.

\section{- Ductile Iron Pipe (DI) Design and Installation}

In 1975, ductile iron pipe was introduced to the pipeline construction industry. Ductile iron has high strength, durability, low maintenance and corrosion resistant (Reed, 2006). The installation of ductile iron pipe is not complicated. It can be installed in various size trench and bedding conditions. Also it can be simply cut in the field. The diameters of pipe vary from 3- to 64 inches. Cement mortar is normally used for lining finish. Detailed installation procedures can be referred to in the Installation Guide for Ductile Iron Pipe by DIPRA (DIPRA, 2001; DIPRA, 2003).

\section{- Polyvinyl Chloride Pipe (PVC) Design and Installation}

Polyvinyl Chloride (PVC) pipe was introduced and available to use for sanitary sewer and water distribution markets in 1950s. The advantages of PVC pipe are lightweight, easy for 
handling, corrosion resistance, chemical resistance, and flexible (Uni-Bell, 2001). PVC pipe can be separated into pressure pipe and non-pressure pipe. The installation procedures of PVC pressure pipe include;

- Trench Construction

- Appurtenances

- Locating, Leak Detection, and Thawing Procedures

- Inspection and Testing of Pipe System

For PVC non-pressure pipe, the installation processes are;

- Trench Construction

- Pipe Laying

- Appurtenances

- Inspection and Testing of Pipe System

Details of PVC pipe installation are also available in the Handbook of PVC Pipe Design and Construction by Uni-Bell PVC Pipe Association (Unil-Bell, 2001).

\section{- Clay Pipe Design and Installation}

Vitrified clay pipes have been present in the pipeline industry for a century. One of the first American clay sewer pipe plants was operated in 1849. Clay pipes are strong, chemical and corrosion resistant, and also heat resistant (U.S.EPA, 2000). An open trench is a typical installation of clay pipe, but microtunneling is also suitable for clay pipe installation. Microtunneling methods offer the least disruptive and the most cost effective method for clay pipe installation. Installation details of clay pipe can be referred to the Clay Pipe Handbook (NCPI, 2004).

\section{- Polyethylene Pipe (PE) Design and Installation}

Polyethylene (PE) pipes were first introduced in the late 1950s. PE pipe is suitable for rehabilitation, narrow trenching and trenchless applications. The advantages of using PE pipe are flexibility, lightweight, toughness, and corrosion resistant (Reed, 2006). The installation techniques for PE pipe vary by location and function of the pipe. 
Underground Installation of PE pipe can be done by trench excavation. Some special installation techniques include, plowing, planting, pulling pipe into a narrow trench, pipe bursting, insertion renewal, horizontal boring, and directional boring which require less excavating. Additional information of PE pipe installation is available in the Manual of Water Supply Practices M55, PE Pipe - Design and Installation by American Water Work Association (AWWA) (AWWA, 2005).

\section{- Reinforced Concrete Pipe (RCP) and Pre-Stressed Cylinder Pipe (PCCP) Design and Installation}

In 1942, pre-stressed concrete pipe was first developed in the U.S. Pre-stressed concrete cylinder pipes (PCCP) are used for force mains, whereas reinforced concrete pipes (RCP) are used in gravity lines. There are two general types of concrete pipe construction; embedded-cylinder (EC) and lined-cylinder construction (LC). The benefits of concrete pipe are high beam strength and rigidity, easy joining system, and corrosion resistant (Reed, 2006; U.S.EPA, 2000). There are three typical types of PCCP installation techniques which are trench, positive projecting embankment, and negative projecting embankment. In Addition, jacking or tunneling method is also applicable where deep excavation is required. Procedures of PCCP installation are available in Concrete Pipe Design Manual by American Concrete Pipe Association (ACPA) (Urquhart, 2006).

\subsection{Existing Condition Rating}

Condition rating, also referred to as condition grading is an evaluation of the infrastructure's current physical state compared to its newly constructed state. Prior to predicting the future condition and performance of an infrastructure asset, it is necessary to first assess its condition. Typically, condition ratings are numerically based and scales have either the highest or the lowest value, which indicates that the asset is in new condition. The lowest or highest rating on the scale also indicates that the assets has failed (Mehle, 2001). The challenge is to provide a combined or overall condition rating for the infrastructure. Most utilities rely on condition assessment based on visual inspections (closed circuit televising (CCTV) and man entry inspections) and expert opinion. Inspectors also use nondestructive testing, such as 
electromagnetic (radar), magnetic, acoustic (leak detection), sonar, smoke tests, and dye tests, when inspecting sewers. Currently, inspectors use three categories of inspection methods, subjective grading, distress-based evaluation, and nondestructive evaluation (Rahman, 2004).

Typical sewer grade is defined by a number from one to five, which is determined by a defect score calculation based on the summation of deduct values for different defects in the pipe segment, usually manhole to manhole. The deduct value is weighted according to condition assessment methodologies using the impact on service life and performance as criteria. Typical scores are calculated using the equations 1 to 3 .

$$
\begin{aligned}
& \text { Mean Score }=\frac{\sum(\text { Deduct Values })}{\text { Length of Pipe Segment }} \\
& \text { Peak Score }=\text { Maximum Deduct Value } \\
& \text { Total Score } \left.=\sum \text { (Deduct Value }\right)
\end{aligned}
$$

The mean score represents an average of deduct values over the pipe segments (manholemanhole), while the peak score represents the maximum deduct value and the total score represents the summation of the deduct values. The current 1 to 5 rating system has been considered not minute enough to represent a reasonable condition state. More minutely classified condition rating will considerably increase the accuracy of performance prediction model of underground infrastructure (Park, 2007).

\section{NASSCO's Pipeline Assessment and Certification Program (NASSCO, 2003)}

The National Association of Sewer Service Companies' (NASSCO) Pipeline Assessment and Certification Program (PACP) is a standardized system used to evaluate internal television inspections of wastewater collection assets. Many utilities in the U.S. have already adopted NASSCO's PACP. Using this system, visual defects are identified based on key structural and operation and maintenance (O\&M) parameters. Collection system assessments, through PACP, or other similar methods, can then be recorded and evaluated. The condition of the wastewater collection system is based on the rate of deterioration of the installed materials. Deterioration 
factors include, but are not limited to, soil conditions, groundwater, hydraulics, surface loadings, installation, and internal conditions (roots, grease, surcharging, etc.). Pipeline defects are also subject to become more severe over time, experiencing events such as deformation, subsidence, or hydrogen sulfide attack (NASSCO, 2003).

PACP distinguishes televised pipelines into the following four categories;

- Structural defects: crack, fracture, broken, hole, deformed pipe, collapse, joint defects, surface damage, lining defect, weld failure, point repair, and brickwork.

- Operational/maintenance defects: deposits, roots, infiltration, obstruction, and vermin.

- Construction features: tap, intruding sealing material, line, and access points.

- Other: miscellaneous observations.

\section{Water Research Center (WRc) Sewer Rehabilitation Manual (WRc, 2001)}

The Water Research Center Sewer Rehabilitation Manual, initiated in 1978, underwent several revisions until the WRC published the most recent version in 2001 consisting of two volumes. Volume 1 addresses the determination of the structural performance of sewers, survey techniques, and procedures for assessing deterioration and collapse mechanisms, analysis of hydraulic performance, and maintenance planning and Volume 2 addresses new renovation techniques and structural design methods for sewer renovation. Additionally, the 2001 version includes current best practices on environmental, operational, maintenance investigation, and computerized grading systems compatible with European defect coding systems and new design methods for renovation techniques.

The data collected and coded during CCTV inspection is used as an input for WRc's internal grading system. This system derives a grading of the sewer based on the observation during CCTV inspections. The WRc method assigns deduct values for both structural and operational defects ranging from 1 to 165 . Once the structural and operations defects rating is assigned, the condition grades are assigned a rating from 1 to 5 for sewer. The WRc recommends using the peak deduct values (worst defect) to determine condition grade. 


\section{Assessment and Evaluation of Storm and Wastewater Collection Systems (NRC-CNRC, CANADA)}

This NRC-CNRC best practice report presents a systematic and proactive approach for managing storm and wastewater collection systems. Within this report are recommended five tasks. First, a detailed inventory of storm and wastewater collection system attributes should be compliant and up to date. Additionally, the database and guidelines should include information on location, physical dimensions, related land use areas, operating conditions, and applicable operational data. Second, the system's operating conditions should be investigated for consistency and accessibility of accurate data. Third, the condition assessment of pipes within the system should be rated based on structural integrity, functional integrity, and hydraulic adequacy. Fourth, on a system-wide basis, the individual pipe should be evaluated for performance for all rating criteria. Fifth, a rehabilitation and replacement plan should be developed following the system's needs. In addition, monitoring, inspection, and detailed assessment of the infrastructure in critical sectors is recommended for sewer rehabilitation and replacement work for renewal programs

The NRC-CNRC report also suggests the condition rating systems developed by IRC (IRC, 2001) and the City of Edmonton, Alberta, Canada. As such, structural defects are categorized according to failure mode and severity and the weight, used to calculate structural condition ratings, is given to each defect type. Finally, various combinations of theoretical load factors, grade line factors, and upstream impacts determine the hydraulic condition rating (FCM and NRC, 2004).

\section{Performance Indicator for Wastewater Collection Systems (Tabesh, 2006)}

The performance of the pipe is defined as its ability to covey sewerage without hydraulic overload, create minimal environmental impact, and retain good structural integrity. To analyze the level of service of sewer collection system, hydraulic performance is the most important parameter. Additionally, hydraulic performance depends on the dimension of the pipe, as well as structural deterioration, such as blockage and roots. According to Tebesh (2006), the performance of a wastewater system is evaluated using two penalty curves, depth, and velocity of wastewater flow in each pipe section, as well as weighting function. 
Results show that indicators, based on flow depth, provide a better representation of hydraulic performance than pipe velocity because of the lower sensitivity of PI. These indices aid in decision- making of the management of wastewater collection system. Tebesh also suggested that in order to better understand the system, a set of hydraulic and mechanical parameters should be considered and the network performance index should be calculated using the weighted means of these parameters over a period of time. Based on Tebesh's findings, future investigations should consider other parameters, such as pipe roughness coefficients and variation of sewer inflow to the system.

Condition Assessment of Buried Pipes Using Hierarchical Evidential Reasoning Model (Bai, 2008)

Bai (2008) proposed an approach to evaluate the condition of a large diameter wastewater pipe based on hierarchical evidential reasoning. Bai implemented the Dempster-Shafer (D-S) rule of combination to combine different distress indicators at different hierarchical levels. Bai also introduced an application called the HER Framework. The HER framework describes the framework of the proposed hierarchical evidential reasoning model and provides evidence into two levels. The elements of basic evidence are referred to as factors, which are combined into attributes or categories for more general evidence. In pipe condition assessments, the aggregation of categories from evaluating distress indicators is used to obtain the overall condition rating. The distress indicators are signs of the aging process, which are dependent on pipe materials, the surrounding environment, and the cumulative effects of stresses.

One of the advantages of the HER model is that this model can handle incomplete or conflicting evidence. Assumptions about missing data are not required as with other probabilistic models. The HER model is built based on contributory factors or attributes that are assumed independent and it is the parallel aggregation of factors or attributes that are executed using the D$\mathrm{S}$ rule. 


\subsection{Existing Prediction Model}

The following literature review discusses actual references and a brief summary of the prediction model used within the paper in addition to the advantages and limitations of the approach or model.

\section{Deterministic Models}

There are five specific articles published on the use of deterministic models for wastewater pipe condition, performance assessment, and prediction modeling, which are discussed below (Ariaratnam, 2001; Chughtai, 2007; Farshad, 2004; Kaempfer, 1999; Whittle, 2005).

\section{- Assessment of infrastructure inspection needs using logistic models (Ariaratnam, 2001)}

Ariaratnam (2001) applied logistic regression modeling, which is a special case of linear regression, to predict the likelihood that a particular infrastructure system was in a deficient state. The difference between logistic regression modeling and linear regression modeling is that logistic regression modeling outcome variables are binary and assume a Bernoulli distribution. The purpose Ariartnam's study this research was to assist decision makers by providing a method to plan an inspection rather than to perform an inspection randomly. The following demonstrated a case study of Edmonton, Alberta, Canada sewer system.

The physical and condition data of the sewer were obtained from Edmonton, which were stored in a computerized database system called Drainage Asset Inventory System (DRAINS). The structural condition of the sewer was rated 1 (best) to 5 (worst) based on the defect type. Sewers with a condition rating of 4 or 5 were classified as deficient and were considered for rehabilitation, whereas those with condition ratings of 1 to 3 were classified as non-deficient. Ariaratnam analyzed all parameters using a two-step model selection. Specifically, Ariaratnam

first examined the significance of each parameter using a Wald Test, and then determined which parameter to include in the logistic regression model using a likelihood-ratio test. The results of these tests indicated the significant parameters that remained in the model, as well as non- 
significant parameters that the researcher removed from the model. Ariaratnam built the final model from three variables, age, diameter, and waste type.

In conclusion, the Ariaratnam's model provides information about the likelihood of system being in a deficient state. This knowledge can aid utility workers in prioritizing inspections. Additionally, well-timed inspections will better detect deficiencies before a failure occurs and will reduce the number of major rehabilitations. Ariaratnam noted that the method presented can be applied to any infrastructure system and the results of the model will only be as good as the quality of data collected.

\section{- Sewer pipeline operational condition prediction using multiple regression} (Chughtai, 2007)

Chughtai (2007) presented the development of a multiple regression model to predict existing operational condition ratings of sewers based on historical condition assessment data. Chughtai noted non-hydraulic and hydraulic as two categories of factors that could deteriorate the overall operational condition of sewer pipes. Non-hydraulic problems include structural condition, random blockage of flow, operational and maintenance strategies, and history. Hydraulic problems occur when sewer capacity cannot sustain the flow volume; a situation that may be influenced by a number of factors. Chughtai's study focuses on the factors that play a major role in operational condition prediction and assumes that the structural condition of the pipe is not known. However, structural conditions of a pipe depend on known factors such as age, size, depth, soil, and traffic conditions. Chughtai (2007) collected data from Pierrefonds municipality, Quebec, Canada. The pipe data was then converted from the Centre for Expertise and Research on Infrastructures in Urban Areas, Canada (CERIU) to WRc protocols, which specify a condition class of 1 to 5 for each pipe section. Chughtai further processed the data prior to applying the data for model development and validation.

The preliminary analysis indicated that the age of a pipe has significant effect on the operational condition of the pipe. Results further showed that the relationship was exponential and the operational condition deterioration rate of an older age pipe was higher than that of a new pipe. Overall, Chughtai's findings suggest a model for the overall operational condition of sewers. 
This model may be used in identifying critical pipe sections that are susceptible to overflow and flooding. In addition, this model is valuable in prioritizing inspection and rehabilitation of a sewer pipe.

\section{- Estimation of service life of concrete pipes in sewer networks (Kaempfer, 1999)}

Kaempfer (1999) presented an estimation of service life of concrete pipes based on theoretical corrosion rate and accelerated laboratory tests. According to Kaempher, high traffic load volumes, improper construction, and sewage engineering were main causes of sewer pipe damage. Exfiltrations into ground water are typically the result of damaged sewer pipes. Specifically, this occurs from the contamination of sulfate, chloride, and nitrogen compounds in the sewer system. This contamination produces the biogenous sulfuric acid, which results in corrosion in concrete and brickwork, also referred to as sulfide generation and corrosion process. In determining the rate of corrosion in concrete pipes, utility workers consider the strength and density of concrete, degree of acid penetration, acid value, and circulation of hydrogen sulfide in the atmosphere. Additionally, the geometry of the pipes and the filling capacity significantly affect the rate of corrosion.

Kaempfer predicted the attack level of biogenous sulfuric acid in sewers based on the calculation using the Pomeroy value. The example in Kaempfer's study showed that concrete pipes need to be protected against acid corrosion when exposed to extreme conditions. Kaempfer developed a test method to simulate resistance to a biogenous sulfuric acid attack. The purpose was to show how sulfuric acid attacks in dirty and combined wastewater systems. Kaempfer weighed waterlogged specimens stored in plastic containers and filled with diluted sulfuric acid ( $\mathrm{pH}$ value 1, 2 or 4.5). Kaempfer then removed and weighed the specimens after 6, 13, 20, 34 days, respectively, for a total $\mathrm{pH}$ bathing process of 35 days.

Result indicate that one of the corrosion types in sewage systems is biogenenous sulfuric acid, the production of which depends on pipe diameter, sewer length, pipe gradient, pipe ventilation, turbulence, and other aspects of construction. Today, sewers are increasing in size and flow distances and periods are longer than in previous years; therefore, utility workers should closely monitor the biogenous sulfuric acid problem affecting pipe service life. The study also notes that using both theoretical equations and time-accelerated laboratory tests have the potential 
to predict corrosion rate. Pomeroy's equation and the observed corrosion of a 22 year-old unreinforced concrete pipe, provided similar results. The time-accelerated test produced good results and showed that diluting sulfuric acid for 35 days with 5 cycles matched real-time stresses seen in 20 year-old combined sewers.

\section{- Two new criteria for the service life prediction of plastics pipes, (Farshad, 2004)}

Farshad (2004) proposed two new criteria for service life prediction of single-layer and multilayer plastic pipelines that are under pressure based on stress, strain, and energy regression analyses. The two methods proposed in this study were the ultimate strain extrapolation method (USEM) and the distortion energy extrapolation method (DEEM). Overall, three models were employed based on stress, strain, and energy-based regression analysis. Additionally, Farshad included sample data from a PVC-U pipe to verify the proposed methods.

The first method, USEM, was based on the use of the failure strain criteria, rather than the typical employed stress concept. The USEM procedure consisted of performing internal hydrostatic pressure tests and then performing creep tests to determine the creep modulus. Next, a calculation of the hoop stress, using the membrane theory or the three-dimensional elasticity theory, was applied. The strain was then calculated in relation to the hoop stress in which the creep modulus was obtained from tensile tests on material samples. Regression analyses were used to determine the long-term failure strain as a function of time.

The other method presented by Farshad, DEEM, utilized the ultimate strain, rather than the rupture stress. The DEEM procedure consisted of performing creep tensile tests as well as performing internal hydrostatic pressure tests. A calculation of the hoop stress, using the membrane theory or the three-dimensional elasticity theory, was then applied. The distortion energy density was calculated using the creep modulus and then the hoop strain was calculated. Finally, regression analyses were used to determine the long-term failure strain as a function of time.

Each model was compared to the standard extrapolation method (SEM). Results indicated that there was a good agreement between the independent model and the modified SEM analysis. 
Overall, the proposed USEM appeared to be suitable for brittle and fiber reinforced materials, while the DEEM was believed to be applicable for a broader range of material types.

\section{- Predicting the residual life of PVC sewer pipes (Whittle, 2005)}

Whittle (2005) described a method to predict the residual life of PVC sewer pipes. The Ipswich Water Department examined the service life of different pipe materials of gravity sewer pipes and discovered that PVC sewer pipes had no failure histories or deterioration as seen by the CCTV inspections during 25 years of service. Therefore, a deterministic approach was considered in order to predict the pipe life rather than using a statistical approach. Yield strength, bending modulus, wall thickness, joint performance and impact resistance were pipe characteristics chosen to determine the quality number. Two series of factors were selected because the outcome was influenced by the selection of the weighting factors.

These tests indicated that no deterioration in the material or the joints met current performance specifications. Additionally, these results were compared with multivariate statistical analyses, which confirmed the life of the PVC pipe to exceed 100 years.

\section{Conclusions}

Deterministic models predict the remaining life or related failure rates to asset attributes of buried pipes. This specific model is often used specifically where the relationship between components are certain. Additionally, there are two different approaches in deterministic models, the empirical approach, and mechanistic approach.

Researchers use the empirical approach in deterministic modeling to develop failure rates of buried pipelines. Models using this approach apply an equation to predict asset failures. These equations can be linear or non-linear and various parameters may be used to best associate failure with the pipe. Historical data combined with these parameters can be separated into different groups to apply the empirical approach for the model, resulting in predicted outcomes from the empirical deterministic models, which are then compared to actual observed events. Overall, the empirical approach in deterministic modeling does not improve actual knowledge of specific causes of 
degradation nor does this method account for deterioration of time, third party damage, or naturally occurring events (Marlow, 2008).

On the other hand, using deterministic modeling with the mechanistic approach, serves to predict service lifetimes of individual assets rather than failure rates of groups. In order to apply such models, the parameters and loading conditions of the water pipe must be known or estimated based on field observations. Overall, this method evaluates the degradation mechanisms of pipes, which affect the overall condition and lifetime of the pipe. This modeling technique is applied to individual assets and must be aggregated in order to analyze the system as a whole since physical models do not allow cohort estimates of pipe failure rates (Marlow, 2008).

Therefore, the summaries of the deterministic model approach are as follows:

-The majority of the existing deterministic-based models may be classified as structural or functional performance models and primary response models.

-The approach can predict an average single value of a dependent variable.

-This approach has two distinct categories: mechanistic and empirical. Which approach is used depends on the dependent and independent variables used in the models and how their relationships are established.

-The development of many existing prediction uses regression analysis combined with mechanistic-empirical analysis and the subjective opinions of experienced engineers.

-The problem of deterministic-oriented prediction models is the applicability of each individual model is restricted to a specific location.

-It is inadequate to apply deterministic models to all situations of pipe infrastructure management because (a) the uncertainties in pipe behavior under various environmental conditions and (b) the difficulties in quantifying the factors or parameters that affect the rate of pipe deterioration. 


\section{Statistical Models}

Six specific articles, published on the use of statistical or probabilistic models for wastewater pipe condition, performance assessment, and prediction modeling, stand out in the literature and are discussed in the following (Baik, 2006; Baur, 2001; Kleiner, 2001; Kleiner, 2004; Korving, 2008; Ruwanpura, 2004).

\section{- Prediction models for sewer infrastructure utilizing Rule-Base Simulation (Ruwanpura, 2004)}

Ruwanpura (2004) developed three models to aid in the planning of sewer maintenance expenditures using a rule-based simulation and probability analysis. This researcher based the proposed prediction model on the Markovian approach, in the City of Edmonton, Alberta, Canada. The proposed models included the following.

-Model 1: To predict the present Condition Rating (CR) of sewer pipes if the age, type of material, and length of pipe are known.

-Model 2: To predict the future CR of sewer pipes if the present condition of the same pipe, age, type of material, and length of the pipe are known.

-Model 3: To predict the present and future costs of renovating sewer pipes based on the outputs of models 1 and 2.

In summary, the models used a combination of rule-based simulation and probability analyses to assist in the planning of future expenditures for sewer maintenance, which could later serve as invaluable planning tools for the City of Edmonton. The applications of these simulations in real life projects are minimal because the construction industry has not yet verified the practical use of these models, despite the fact that this is a very powerful tool for decision-making purposes. This article describes one way of implementing the simulation in prediction when extensive historical data is not available. Ruwanpura also found that the measurement of the structural rating system might cause erroneous decisions. Ruwanpura also raised a number of questions with respect to the assessment of $\mathrm{CR}$, suggesting the development or revision of CR for the City of Edmonton. 


\section{- Selective inspection planning with aging forecast for sewer types (Baur, 2001)}

Baur (2001) used a cohort survival model to predict the required time to reach the critical condition category for a sewer pipe. Since Germany requires that sewer pipe networks are inspected every 10 years, costs for this regular inspection are relatively high due to the expected life time of the network. The current conditions of sewer pipes are a necessary component to forecasting sewer service life; therefore, observing sewer conditions assist decision makers in making timely decisions concerning this system. As such, Baur's investigation used a cohort survival model to predict time to reach the critical category of condition in sewer pipes.

Baur developed a software package (AQUA-WertMin) for sewers using special distributions called Herz distributions. The software package allows utility workers to explore strategies for asset management, especially in the examination and calculation of financial requirements for asset management based on condition thresholds.

In the case study, Baur modeled the Dresden sewers in order to find the aging speed of different sewer types as well as to determine this factor's application in inspection planning. The Dresden sewers have been categorized as a 5 in terms of condition classes (cc), with 5 meaning in very good condition and 1 meaning in very poor condition and being first priority for rehabilitation. Baur aimed to predict how long it would take a sewer to enter a predefined critical condition class. A determination of the aging speed from previous inspection data may be shown as either faster or slower than the average; however, without previous inspection data, as was the case in this study, the average aging behaviors of the sewer type were assumed. Baur calculated the parameters of the Herz transition function using the weighted least squares method. AQUAWertMin determined transition dates and residual lifetimes, ending with transition into cc1 and the aging speed of inspected sewer.

Baur's pilot study applied the cohort survival model to the Dresden sewer network and described the process of sewer deterioration using transition functions into successively worse condition classes. Sewers with specific characteristics deteriorate much faster than others do; therefore, they require shorter inspecting intervals. Baur's study also suggested determining the first inspection time using the median age of transition into this condition class. In doing do, utility 
worker could plan successive inspections based on previous inspections in order to find the aging speed of that particular sewer. This process would result in decreasing the risk, while reducing the costs by providing proficient-selective inspections.

\section{- Bayesian updating of a prediction model for sewer degradation (Korving, 2008)}

Korving (2008) implemented the Bayesian prediction model into SPIRIT, a model developed in the Netherlands for predicting the condition of sewers, specifically sewer degradation. This model was developed based on expert knowledge and inspections based on predicting sewer condition 5 and 10 years ahead of the given current conditions of the sewer. The Bayesian analysis was used to update the prediction model taking into account uncertainty due to a lack of data, with that caveat that distributions can be updated when observations are available. This study also applied the Bayesian approach to an example. The Dirichlet distribution was used to express subjective prior knowledge that was updated using inspection data. The example observed cracks and fractures based on EN13508-2, and used subjective data including expected probability of condition states and weight of prior information expressed as "virtual observations."

\section{- Estimating transition probabilities in Markov chain-based deterioration models for management of wastewater systems (Baik, 2006)}

Baik (2006) described a method to estimate the transition probabilities of different condition states in Markov chain-based deterioration models for wastewater systems, using an ordered probit model and incremental model (as proposed by Madanat, 1995). The changes in condition ratings during a transition period were calculated as discrete outcomes in the ordered probit model. After the transition probability matrix was obtained, the expected condition of the system in the future was easily determined.

This proposed model was applied to the sewer condition data from the City of San Diego's Metropolitan Wastewater Department (MWWD). The condition rating system used in Baik's study was based on MWWD's condition ratings for the inspection of these sewers, which consisted of 108 criteria, categorized into seven subgroups. Each subgroup contained rating criteria representing the characteristics and severity of the defects. Maintenance and structural points were 
assigned to evaluate the conditions of the sewers and the condition rating scores were calculated using the structural maintenance points shown in equation 4.

$$
\text { Score }=\frac{\sum S P \times S W+\sum M P \times M W}{L S}
$$

Where; $S P=$ structural points, $S W=$ structural weight, $M P=$ maintenance points, $M W=$ maintenance weight, and $L S=$ length of segment $(\mathrm{ft})$.

\section{- Scheduling inspection and renewal of large infrastructure assets (Kleiner, 2001)}

A decision framework was introduced by Kleiner (2001) to assist municipal engineers in optimizing the renewal of large infrastructure asset decisions. The proposed methodology used a semi-Markov approach to develop prediction models and expert opinions to determine model parameters. The proposed decision framework indicated the need for immediate intervention or, enabled the optimization of scheduling for future inspections and condition assessments. The semi-Markov process was used as the deterioration model and separated into condition states. The conditional probability allowed all transition probabilities $p_{i, i+1}$ to populate the transition probability matrix for the semi-Markov process.

Random waiting times were assumed as known probability distributions and calculation of cumulative waiting times were obtained from a Monte Carlo simulation. The waiting time $t_{i}$ of the process, in any state, $i$, can be modeled as a random variable with a two-parameter Weibull probability distribution. Based on historical observations and condition assessments of large buries assets, data are currently insufficient data to obtain parameters $\lambda_{i}$ and $\beta_{i}$. Because of these insufficiencies in municipal data, analyses require the development of information based on expert opinion. Therefore, these parameters, in Kleiner's study were derived from expert opinion. This research suggests that the expert must answer questions based on their beliefs about the probability of an asset remaining in a given state for a certain period of time. Once parameters $\lambda_{i}$ and $\beta_{i}$ were established for every $i=\{1,2, \ldots, n 21\}$, the transition probability matrix could be calculated by substituting (2) into (1). 
An update of these distributions, based on the observed deterioration data over time, was collected and the total expected discounted cost was used to determine the time to conduct the next inspection and condition assessment. When the discount cost was less than 2 to 3 years, the next inspection should have been planned immediately. Kleiner also identified some issues for further study concerning the development of a framework as well as the use of undetermined data into the full application tool. Finally, Kleiner presented a hypothetical example case, which showed a systematic procedure to obtain a transition probability matrix, which could be further used to predict the deterioration of the sewer.

\section{- Modeling failure risk in buried pipes using fuzzy Markov deterioration process,} (Kleiner, 2004)

Kleiner (2004) examined the possibility of modeling failure risk in buried pipes using a fuzzy Markov deterioration process. The possibility of failure was analyzed along the entire life of the pipe. Based on Kleiner's results, this model can be used to predict future deterioration rates of an asset. In this specific model, triangular fuzzy numbers were used represent the data or variables. Additionally, Kleiner defined these triangular fuzzy numbers using three points on a graph, representing the vertices of the triangle. Fuzzy rule-based modeling was also used to represent the means of fuzzy if-then rules. The rule set for this specific example utilized the command if the pipe age is " $\mathrm{A}$ " and the pipe condition state is " $\mathrm{C}$ " then the deterioration rate is "D" at any given time.

Overall, the deterioration was separated into two different steps. The first step is completed using the following equation.

$$
\mathrm{D}^{\prime} \mathrm{t}=\left(\mathrm{At} \mathrm{t}^{\wedge} \mathrm{Ct}\right) \text { o RD }
$$

Here, $A_{t}$ and $C_{t}$ are the age and condition state of the pipe at time $t$, which are inputs. $\mathrm{R}_{\mathrm{D}}$ is the fuzzy-rule set and $\mathrm{D}_{\mathrm{t}}{ }_{\mathrm{t}}$ is the output or deterioration at time $t$. The age at time $\mathrm{t}, \mathrm{A}_{\mathrm{t}}$, is the values, which are fuzzified. The resulting values of the deterioration, $\mathrm{D}_{\mathrm{t}}$, can then be defuzzified to a value representing $\mathrm{D}_{\mathrm{t}}$. The second step evolved the deterioration process is described in the next equation. 


$$
\mathrm{Ct}+1=\mathrm{Ct} \Theta \mathrm{Dt}
$$

Here, $\mathrm{C}_{\mathrm{t}}$ is the condition at year $t, \mathrm{D}_{\mathrm{t}}$ is the deterioration rate in the previous equation, and $\Theta$ is an operator. This equation yields membership values in which deterioration models can be created. Once the model is generated, it must be trained in order for the model to yield valuable results. As such, this model is trained to predict future deterioration using condition states of an asset immediately following installation, which are then verified to evaluate the prediction.

The membership values associated with the failed condition can also be viewed as the possibility of failure. This possibility of failure, within this example, was separated into nine different grades from extremely low to extremely high. Another fuzzy-rule based model was also generated to analyze the possibility of failure. These values could then be used to generate fuzzy risk levels over the life of a pipe from the fuzzy possibility of failure and the failure consequences. This fuzzy Markov model is one method that could be used to generate future deterioration rates with a lack of pipe data and vague information concerning pipe conditions.

\section{Conclusions}

Researchers typically use statistical modeling to predict future conditions and performance and will most often use statistics to create a model based on observed data in order to serve as a tool or mechanism to describe such data. Based on statistical techniques, researchers can construct a model to estimate parameters. Additionally, one can use statistical modeling to separate information into two main distributions; discrete random variables and continuous random variables.

Both discrete and continuous distributions can also be separated into more specific distributions including binomial, multinomial, hypergeometric, geometric, Pascal, negative binomial, and Poisson. Distributions, which are applications of continuous statistical information, can be further classified as normal, gamma, exponential, beta, uniform, log-normal, Rayleigh, Cauchy, Weibull, and extreme value. Additional information regarding different types of statistical distributions can be referenced in Hahn (Hahn 1994). 
Empirical modeling consists of five steps: collecting data, analysis of data, model selection, parameter estimation, and model validation. A challenging step in this process is selecting a suitable model, especially since several models may be applicable. Additionally, the model selected must be ample enough to model the data with ease as well as analyze the data with a sense of complexity. Analyzing graphical methods of the model selected is one way of checking the validity of the model (Murthy, 2004).

Probabilistic modeling involves the use of statistical analysis to analyze the probability or relative frequency of an event occurring. Each event results in a random variable, which describes an outcome of a random experiment (Creighton, 1994). The term random is generated since the result is previously unknown to the experimenter. A simple illustration of a probability of a certain event, such as “A," occurring can be denoted by P(A) (Mitrani, 1998).

Therefore, the following apply concerning the statistical model approach:

-This approach can predict a distribution and range of values for dependent variables, such as pipe condition state vectors.

-This approach is applied more often in pavement, bridge, and other infrastructure network management concerning repair, rehabilitation, and replacement priority programming.

-Using regression modeling, the dependent variable of pipe conditions or some other indicators are related to one or more independent variables such as soil, thickness, load, etc.

-Conditions of pipe rankings are required to predict future condition.

-The statistical model can be applied to homogenous groups of a pipe infrastructure system.

-One common feature among different types of statistical models is the large amount of long-term observed field data and the fact that they are processed through regression analysis.

-In many cases, the regression-based approach is not suitable for modeling actual deterioration processes of pipe infrastructure because the sampling data used in the 
regression analysis suffers from various limitations, such as pipe structure, loading, and other environmental variables.

-This model is established based on extensive data.

\section{Heuristic Models}

Three notable articles, published on the use of Heuristic models for wastewater pipe condition, performance assessment, and prediction modeling include those by Bengassem (2000), Najafi (2005), and Najjaran (2004).

\section{- Fuzzy expert systems for deterioration modeling of buried metallic pipes (Najjaran,} 2004)

Najjaran (2004) proposed expert systems for predicting deterioration rates of buried metallic pipes based on surrounding soil properties. An expert system uses information based on expert knowledge. In general, the combination of theoretical knowledge and extended observation forms expert knowledge. Providing additional information from the input-output data of a real system, field data, acquired during inspection, repair, and renewal, provides more precise and specific information of a system; however, to obtain field data for the entire pipe network is impractical. Therefore, fuzzy inference models, which can integrate information from human experts and field data is an appropriate approach in developing a pipe deterioration model.

Najjaran proposed a fuzzy logic expert system comprised of two modules. The first module, knowledge base, is a fuzzy inference model developed from fuzzy if-then rules. The second module, an inference mechanism, is formed using fuzzy reasoning methods to process the knowledge base and deduce an output for instantaneous input.

The knowledge base of this system comes from two sources, expert knowledge and field data, which consists of a subjective and objective model. The first part is the subjective model that provides a fuzzy relationship between soil properties and corrosive potential $(\mathrm{CoP})$. A quantitative relationship between the input and output variables is not required in the subjective model. Rather, the subjective model is generated using fuzzy inference modeling based on the literature and expert surveys. On the other hand, the objective model requires a measureable 
quantity output, for example breakage frequency and maximum pit depth. This model can also be formed using either fuzzy inference modeling or a regression analysis of field data.

The inference engine of expert systems consists of two fuzzy reasoning algorithms. Mamdani's reasoning uses minimum operators as their t-norm and logical reasoning uses product operators as their t-norm. With regard to the objective model developed previously, a reasoning method should be chosen based on the closest fit to the field data. The defuzzification of the proposed fuzzy logic expert system is completed using the height method in which elements at the center of the area are calculated based on elements that have a membership grade of $\alpha$. Where $\alpha$ $=\mu_{\max }(\mathrm{y})$; here $\mathrm{u}(\mathrm{y})$ represents the output membership function in the output universe of discourse.

Najjaran tested the proposed expert system to predict the corrosive potential for a given soil sample using soil properties, pipe age, and maximum pit depth. Najjaran then used a linear regression model to relate the corrosive potential to deterioration rate (DR) (see equation 7).

$$
D R=m \cdot C o P+d
$$

Where; $\mathrm{m}=$ the slope of the line,

$$
\mathrm{d}=\text { the intercept of the line }
$$

Equation 8 was calculated based on the available data using the least square method. The mean absolute error for the linear fit was 0.08 and the coefficient of determination $\left(\mathrm{R}^{2}\right)$ was approximately 0.34 .

$$
D R=0.041 C o P+0.026
$$

Based on these results, the corrosive potential was correlated with the deterioration rate; therefore, the corrosive potential can be considered as only one parameter affecting the deterioration of the pipes. Researcher may also use the corrosive potential in a cost benefit analysis to find the optimal level of corrosion protection required. 


\section{- Pipeline condition prediction using neural network models (Najafi, 2005)}

Najafi (2005) proposed a method for predicting the pipeline condition based on historical data using Artificial Neural Networks, intended for the Sewer System Evaluation Survey (SSES) data for the City of Atlanta. This proposed model will help utility workers prioritize the risk of failure in a pipeline when an immediate action is needed. Additionally, this information may assist in determining the feasible condition of a specific pipe and network, the weakest spot in the network, ranking procedures for the inspection project, potential budgeting concerns, and the best possible solution for underground asset management.

Deterioration models are necessary because the determination of cost-effective maintenance solutions require information on current conditions as well as the anticipation of future conditions (Najafi 2005). Furthermore, researchers can predict future failure using deterioration models based on present data collection methods. There are many factors that deteriorate the pipeline condition including, construction failure, local external factors, and other factors (e.g., age, characteristic, maintenance method). Najafi determined deterioration of pipeline conditions based on seven variables including pipe material; pipe age; pipe diameter; pipe section length; depth of cover, slope, and gradient; and sewer type.

Najafi conducted training and testing to develop the Neural Network Model. Specifically, the training replicated a sample set to the network, verified the response, and rectified the answer. The testing followed the same procedure as the training; however, during testing Najafi inputted the hidden sample into the neural network without the correction. Further, Najafi used BrainMaker and NetMaker to create the Neural Network model, with $85 \%$ training and $15 \%$ testing divided randomly using NetMaker preference to the dataset. Figure 4.18 illustrates the typical NetMaker window. When the data preparing process is completed, the modeling process may begin.

Results suggest that Neural Network models used to illustrate pipeline conditions may be able to predict the deterioration of a pipeline, although Najafi's results of this pilot experiment were inaccurate. As such, in order to improve a model, more information must be provided. When developed correctly, this tool can be offer an advantage to assist the decision maker in the evaluation of pipeline or network conditions. 


\section{- Fuzzy expert systems for sewer network diagnosis (Bengassem, 2000)}

Bengassem (2000) proposed a method to evaluate the structural and hydraulic conditions of a sewer system using a fuzzy inference system. The structural inspection and hydraulic simulation, included in the method, evaluated the condition of components in the sewer network. Additionally, Bengassem applied a fuzzy expert system for each pipe segment to combine all factors and determine a performance assessment of the sewer network. Three aspects of this structural performance evaluation included (a) intrinsic (pipe defect), (b) extrinsic (pipe characteristics and environment characteristics affecting a pipe, such as geotechnical factors, hydro-geological factors, and seismic activity), and (c) site vulnerability (nature of site, soil density and others). A score of 0 to 100 was given to each pipe segment based on these three aspects. Additionally, hydraulic performance was evaluated, focused on the conveyance capacity of the system, and assessed using Bennis' formula (Bennis, 1999) to find the surcharge responsibility factor of a sewer pipe.

Bengassem developed three fuzzy systems, including a structural system (FSS), a fuzzy hydraulic system (FHS), and a fuzzy global system (FGS), to evaluate the level of performance. Finally, Bengassem used these fuzzy systems to calculate the performance index (structural performance index and hydraulic performance index). The FGS sums of the different factors formed the global performance index (GPI) for each pipe in the sewer system (Ana, 2007).

\section{- An asset residual life prediction model based on expert judgments, (Wang, 2008)}

Wang (2008) examined the use of expert judgments to analyze residual lifetime predictions of a pump. This study used stochastic filtering theory to predict the remaining life based on experts who monitored condition parameters and treated these parameters as random variables that could be modeled using probability distributions. Since parameters affecting the pump may contain some noise, the proposed methodology of using expert judgments can also be used to reduce noise. There are some limitations when using expert judgments, including inconsistency of expert judgments, lack of experience in making judgments, and inability for the judgment to predict the residual life of a pump, even though a judgment can illustrate the possible state of a pump. 
Wang; therefore, based model expert judgments following assumptions:

-The asset is monitored regularly at specific points using measured monitoring parameters.

-Expert judgments are based on a set of integer numbers such as 0 to $n$ where 0 implies the item is in good condition while $n$ indicates the item has failed.

-When the item is above 0 , the residual life becomes shorter as the number score of the judgment increases.

-The expert judgment is based on the current measured monitoring parameters at the time of judgment.

To begin a model based on expert judgments, Wang formed a probability density function using random variables of residual life at time $t$, conditional on random variables of the expert judgments made at time $t$. The condition that no monitoring parameters were available was then classified using a Weibull distribution function and, assuming the condition that there are expert judgments, classical discrete Poisson and Binomial distributions may be used. In order to estimate the parameters, based on expert judgments and residual life information, Wang applied the maximum likelihood method.

Overall, Wang used three types of data in this study, an artificial data set, simulated data set, and real data set, in order to test the likelihood function. The artificial data was the first set of data used and consisted of the time of monitoring, expert judgments at the time of monitoring, and final failure times. Next, Wang used the simulated data, which estimates parameters to compare to the artificial data set. "At each monitoring point $t_{\mathrm{i}}$, if the expert judgment is more than 0 from the artificial data, generate a $Y_{i}$ based on $x_{i}$ and the estimated parameters according to the distribution of $\mathrm{P}\left(\mathrm{Y}_{\mathrm{i}} \mid \mathrm{x}_{\mathrm{i}}\right)$ since $\mathrm{x}_{\mathrm{i}}$ is known".

Results revealed that the estimated parameters from the simulated experiment did not correlate with the artificial data set. The reasoning for these results may include the fact that there was no correlation between $Y_{i}$ and $x_{i}$, which were the random variables of the expert judgments and residual life at time $t$ or, simply, the nature of the distributions. In conclusion, there was a correlation between $\mathrm{Y}_{\mathrm{i}}$ and $\mathrm{x}_{\mathrm{i}}$, affected by the distribution the artificial and simulated data. Wang 
also found that the variances of Poisson and Binomial distributions increased as residual life decreased. This case cannot be valid since the variance of expert judgments should be smaller because residual life decreased. Therefore, a normal distribution replaced the Poisson and Binomial distributions since the normal distribution did not increase when the residual life decreased. However, findings indicated a correlation between the simulated data and the artificial data set. Wang also conducted a goodness-of-fit test based on X2, which accepted the model at a $95 \%$ significance level.

Wang verified the expert judgments' predictions of residual life using real data from three raw water pumps in a large soft-drink company in England. These expert judgments were classified into four integer numbers as listed below 0-The pump is operating normally.

-1- The pump is operating and shows signs of deterioration. It is advisable to take some preventive action at the next planned maintenance.

-2-The pump is operating, but requires immediate attention.

-3-The pump has failed.

It should be noted that when maintenance occurred, the expert judgment most always went back to 0 from a previous judgment. To capture these results, Wang plotted the probability density function of residual life versus the residual life with and without expert judgments. As illustrated in the graph, as the pump generates expert judgments, the model resembles different patterns. The shape of the probability density function, with expert judgments, also illustrates a more gradual failure over time with smaller variance.

\section{Conclusions}

The heuristic models are more common for large and poorly understood infrastructure problems. Additionally, this type of modeling produces sub-optimal solutions. One can create heuristic models through subjective opinions from experienced field engineers and experts.

The most commonly used techniques in advanced mathematical modeling are artificial neural networks and fuzz logic. Artificial Neural Networks (ANNs) are used to model deterioration and failure of pipes. These neural networks are composed of highly interconnected 
processing elements called neurons that work together to find a solution and are organized in such as a way to perform a particular output. Each of these processing elements is rather simple in nature; however, can become complex if one interconnects several or many networks together. Overall, ANNs are inspired by the way information in a biological nervous system is processed due to its highly complex, nonlinear properties, and parallel computer processing.

Artificial Neural Networks offer many useful properties and capabilities including nonlinearity, input-output mapping, adaptivity, evidential response, contextual information, fault tolerance, very large scale integrated implementability, uniformity of analysis and design, and neurobiological analogy (Haykin, 1994). In summary, developers can train ANNs to be very effective tools in terms of modeling deterioration or failure of water pipes.

Fuzzy logic is based on the way the brain deals with inexact information, as such, neural networks are modeled after the physical architecture of the brain. Fuzzy systems and neural networks are numerical model-free estimators as well as dynamical systems that share the common ability to improve the intelligence of systems working in uncertain, imprecise, and noisy environments (Lin, 1996). Both fuzzy systems and neural networks have been shown model complex nonlinear processes to arbitrary degrees of accuracy. Although fuzzy systems and neural networks are formally similar, there are also significant differences between these two components. For example, fuzzy systems are structured numerical estimators. These systems begin from highly formalized insights about the structure of categories found in the real world and then articulate fuzzy if-then rules as a type of expert knowledge. Fuzzy systems also combine fuzzy sets with fuzzy rules to produce overall complex non-linear behaviors. On the other hand, neural networks are trainable dynamical systems whose learning, noise tolerance, and generalization abilities grow out of their connectionist structures, dynamics, and distributed data representations.

There are several limitations, which should be noted when constructing a fuzzy expert system. For example, an engineer must be knowledgeable in various computer languages and know the basics of constructing a fuzzy expert system. Once the fuzzy system is developed, there 
are the tasks of handling debugging, calibrating, and validating the expert system. Overall, the advanced mathematical model approach encompasses the following:

- The immense capabilities of the human brain in processing information and making instantaneous decisions, even under very complex circumstances and under uncertain environments, have inspired researchers in possibly mimicking the computational abilities called neural networks.

- Topology corresponds to ordering and organizing nodes from the input layer to the output layer of a network. In fact, the way nodes and corresponding interconnections are arranged within the layers of a given ANN determines its topology. The decision to use a given topology is dictated primarily by the type of problem being considered.

- High levels of skill and training are required to develop and train these complex networks. Without this level of skill, a black-box approach occurs.

- Well labeled data is required for supervised training and prediction of future conditions of a pipe.

- Research has found applications in a number of areas of infrastructure management, such as bridges, highways, oil and gas pipelines, and water pipe networks.

- Challenges exist in constructing fuzzy rule sets, selecting membership functions, and deciding defuzzification processes.

\section{Other Models}

\section{- Sustainable management of leakage from wastewater pipelines (DeSilva, 2005)}

DeSilva (2005) described the development of decision support tools to prioritized rehabilitation of wastewater pipe networks, which accounted for leakage of Australia and associated European partners. Leakage is seen as a function of pipe deterioration in modeling the decision support system. DeSilva also presented a deterioration model for wastewater pipelines that were utilized in a decision support system. Closed Caption Televising inspection data has become a major source of information on wastewater pipe quality; however, in order to extrapolate such information to assess pipe deterioration and estimate failure probabilities requires great effort. 
Finally, DeSilva discussed attempts to model this deterioration using CCTV, inspection data, and other environmental data. The deterioration model comprised of four separate models including the exfiltration model, infiltration model, blockage model, and structural model.

In model development, CCTV data that correlates with soil and terrain data were utilized to identify chemical and mechanical reactivity and water retention properties of the pipes surrounding environment. These parameters were used to rank the areas based on the likelihood of ground movement and pipe material deterioration. For the assets that CCTV information was not available, the generic data was populated by soil data and available CCTV data. Soil type and pipe characteristics (material, size, age, slope, and depth) assisted in quantifying the size of defects in joints and pipe fabric. These defect sizes were used in independent models to estimate infiltration and exfiltration based on flow volumes and ground water levels. The probability of blockage was also addressed in multicriterion tools and calculated based on pipe characteristics, root penetration data, and hydraulic modeling.

\section{- Computer aided rehabilitation of sewer networks: Decision support tools for sustainable water network management (CARE-S, 2002)}

The European Commission developed CARE-S with the goal to establish a framework for sewer network rehabilitation decision-making by producing a Decision Support System (DSS) that will enable municipal engineers to establish and maintain effective management of their sewer networks. CARE-S takes into account all aspects of rehabilitation decisions with a link to Performance Indicators (PI). These PI tool handle information relevant to wastewater and storm water network rehabilitation. The PIs were developed based on the information from 32 project partners and end-users. In total, there are 41 PIs with a large number of Utility Information (UI) and External Information (EI).

Analytical and statistical tools used to assess and forecast some of the PIs were developed as well. Additionally there are structural condition tools that are comprised of network level models and specific-pipe level models for corrosion, load-bearing capacity, blockage, in/exfiltration. Socio-economic and environmental risks of malfunctioning wastewater systems were also taken into account in the study. Finally, the researcher proposed a prototype software 
package that supports user decision making in choosing an appropriate rehabilitation technology planning strategy (CARE-S, 2002).

\subsection{Utility Current Practices}

The utility current practices presented in this section were summarized from questionnaires and interviews with the participating utilities. The size of the utilities ranges from major cities to smaller towns. They are located from the east to the west coast to represent the utility current practices in the US.

\section{Atlanta, Georgia}

There are approximately 40,000 manholes and 1,900 miles of sewer mains and laterals within the right-of-way and easements of Atlanta's wastewater collection and transmission systems. The combined sewers are estimated to be $85 \%$ of the system and the remaining are separate sanitary sewers. The cities of Hapeville, College Park, and East Point, and DeKalb, Clayton, and Fulton counties are six other entities that have a wastewater treatment contract with the city. Atlanta generates $55 \%$ of the sewage flow and wholesale agencies generate approximately $45 \%$. The total population of 1.6 million citizen benefits from this system (Hutchinson, 2007). In 1999, Atlanta entered into the First Amended Consent Decree (FACD) with the EPA. The FACD requires Atlanta to implement many of the programs associated with EPA's widely discussed wastewater collection system management initiative for capacity, management, operation, and maintenance, also known as CMOM.

\section{- Condition assessment and rehabilitation practices}

Atlanta will inspect every foot of the pipe system during the condition assessment program titles, Sewer System Evaluation Survey (SESS). The goal of the SSES is to build a complete inventory of the wastewater system, identify sources of Inflow \& Infiltration, and perform a condition assessment. Atlanta's sewers have been categorized into 6 groups and the criteria for the initial prioritization of the Sewer Groups has taken into account ten items that are related to (a) frequency of overflows, (b) severity of rainfall dependent Infiltration/Inflow (RDI/I), (c) risk to surface waters (creeks), (d) impact failures, (e) status of ongoing rehabilitation or renewal, (f) 
available capacity in sewers, (g) judgment of sewer operation and maintenance division, (h) relative impact of RDI/I from jurisdictions outside the city's control, (i) proposed development intensity, and (j) location of sewer within the combined system. The city will study six sewer groups over the FACD's 14-year compliance period and the findings from each SSES study will results in an appropriate prioritized list of identified and scheduled remedial actions for sewers, manholes, and laterals (Hutchinson, 2007).

\section{- Sewer pipe condition assessment practices.}

With this program, sewer inspections identify defects and sources of I\&I including, CCTV, smoke test, dye test, flow monitoring, rain monitoring, building service connection location and inspection, and flow isolation. Defects are rated based on Atlanta's in-house defects rating, called an internal condition grade (ICG), which assigns a rating of 1-5 for each pipe section (see Table 2.1).

\section{Table 2.1 Internal Condition Grade (ICG)}

\begin{tabular}{ll}
\hline ICG & Typical Defect Description \\
\hline $\mathbf{5}$ & Pipe or Brick Sewer already collapsed; or, \\
& Deformation exceeds $10 \%$ and pipe is broken; or, \\
& Extensive areas of material missing; or \\
& Deformation exceed $10 \%$ with fracture(s); or, \\
& Extreme loss of concrete/mortar \\
& Pipe or Brick Sewer is broken; or, \\
& Deformation <10\% with fracture(s); or, \\
& Mutiple fractures; or, \\
& Serious loss of gradient; or, \\
& Severe concrete corrosion; or, bricks displaced \\
& Pipe or Brick Sewer is fractured with deformation between $5 \%$ and 10\%; or, \\
& Longitudinal cracking or mutiple cracking; or, \\
& Severe joint defects; or, \\
& Badly made connections; or, \\
& Moderate concrete corrosion; or, \\
& Bricks displaced \\
& Light corrosion; or circumferential cracks; or moderate joint defects \\
& No corrosion or structural defects \\
&
\end{tabular}




\section{- Sewer pipe repair/rehabilitation/replacement practices.}

GIS, CCTV videos, smoke testing photos, manhole inspection reports, spill reports, hydraulic modeling, and rehabilitation costs are stored in the SSES Raw database, which is used on conjunction with defect ratings to support decision-making. The designer determines the main rehabilitation approach according to the guidelines, which are used to identify the approach to rehabilitation using the Rehabilitation Selection Tool (RST). The main rehabilitation is based on the presence and frequency of a group of essential structural defects such as breaks, holes, multiple fractures, deformations, and large joint displacements. Extensive service defects, such as multiple root masses and root taps, may also warrant rehabilitative actions.

With the RST, engineers categorize projects into Capacity Relief Projects; Rehabilitation; Capacity assessment, Maintenance, Operation \& Management (CMOM); Deferral; and No Rehabilitation. The Capacity Relief Project categories are specified for all trunks and large sewers (greater than 24-inch diameter). Rehabilitation is the main category and is specified for trenchless rehabilitation as well as traditional open cut replacement methods. This category also includes root removal, cleaning, and manhole rehabilitation and replacement. Additionally, CMOM and Deferral is used for sewers in which action is deferred and sewers that need to be monitored for a period of time before a final rehabilitation decision can be made. In cases where only minor defects or no defects are present, the sewers are placed in the No Rehabilitation category (Hutchinson, 2007).

\section{Pittsburgh, Pennsylvania}

The Pittsburgh Water and Sewer Authority (PWSA) was founded in 1984. In 1995, the City of Pittsburgh's Water Department became a part of The PWSA and took responsibility of operating and maintaining the entire City of Pittsburgh's sewer system in 1999. PWSA serves approximately 250,000 consumers throughout the City of Pittsburgh.

Recently, the PWSA proposed the CSO program, which aims to identify cost-effective CSO control alternatives that, when fully implemented, protect water quality. The development of this program requires the monitoring and sampling of plans that propose data collection and 
characterization activities to be included in CSO's Long-Term Control Plan (CSO-LTCP). The CSO-LTCP is the characterization of the combined sewer system operation and the assessment of CSO impacts on river and stream water quality during wet weather events. This Monitoring and Sampling Plan presents the proposed data collection and characterization activities to be undertaken.

\section{- Condition assessment and rehabilitation practices}

The PWSA team developed a CCTV Inspection Program that involves the scheduling of inspections for critical sewers. A review of the sewer system mapping has been completed to highlight the sewers that will be modeled, including the preliminary flow monitoring locations. Approximately 80 miles of sewer mains are designated for televising and individual sewer segments within each of the watersheds have been preliminarily identified for this CCTV investigation. The development of the sewer segment list will be a working document and as additional information is gathered from the sewer investigation field activities and through discussions with PWSA Staff, the sewer segment selections will be amended. If sections of the main truck line sewers are scheduled for rehabilitation or replacement, or if recent televising work has been completed, those sections may not need to be televised during this project. Video data tapes, digital video data on CD-ROM, and television logs will be created for each of the televised sewer segments (PWSA, 2003).

\section{- Sewer pipe condition assessment practices.}

CCTV still frame video images and video clips in a standard video image format will be transferred to the GIS system for use in the InfoNet Commercial Software Database. All staff responsible for rating and evaluating the sewer system conditions shall be NASSCO certified.

The selection of the CCTV segments is based on the following criteria:

-The field inspection program findings suggest that an operational or maintenance issue exists within the system that may affect the hydraulics.

-Record drawings or other information necessary to reconcile the understanding of those sewer networks that do not exist or where information cannot be obtained. 
-Inspection of the pipe is needed to satisfy the requirements of the forthcoming CSO consent order.

The CCTV condition assessment program targets areas identified as critical sewers. Additionally, these areas, as well as additional areas required to clarify system boundaries and connectivity, are included in model development.

\section{- Sewer pipe failure prediction.}

The primary objectives of the hydrologic/hydraulic model of the PWSA collection system are to:

-Characterize the hydraulic response of the sewer system during a variety of wet weather events and calibrate modeled versus metered flows.

-Facilitate the analysis of CSO (combined sewer overflow) control alternatives. -Predict pollutant loadings to the receiving waters.

Once this model is developed and calibrated, it is used as a predictive tool to support CSO abatement planning efforts.

\section{Seattle, Washington}

Seattle Public Utilities (SPU) owns and operates the wastewater collection system for the city of Seattle, which is comprised of 1,491 miles of combined sewer and sanitary pipelines. The wastewater from the city is treated at King County sewage treatment plants. The city's wastewater system services 570,000 people (U.S.EPA, 2005). Seattle Public Utilities' management system includes a 20-year comprehensive plan that provides a long-term direction setting, a 3-year strategic plan that sets the objectives and targets in-line with the comprehensive plan, and specific management systems that support the objectives and targets of the strategic plan (U.S.EPA, 2005). The system plan as set in 2006 and has focused on describing all of SPU's existing wastewater policies and identifying areas where additional policies need to be developed; identifying levels of customer service to minimize sewer backups, street flooding in combined sewer areas, control of combined sewer overflows, and emergency responses; presenting strategies and an 
implementation plan to meet the established customer service levels; and establishing a financial program to fund the programs and activities outlined in the plan (Brown and Caldwell, 2006).

\section{- Condition assessment and rehabilitation practices}

Seattle Public Utilities began the implementation of an Asset Management program approximately five years ago with the initial goal to inventory the existing pipe infrastructure and develop a modeling methodology that would provide the foundation for a successful risk-based replacement and rehabilitation strategy. The immediate goal was to minimize the risk that infrastructure failures were likely bringing to the utility (Martin, 2007). At present, SPU condition assessment decisions are based on the Sewer Pipe Risk Model, an in-house developed model. Fundamentally, the Sewer Pipe Risk Model calculates the risk costs of failure for each individual pipe by multiplying the estimated consequence of failure by the estimated likelihood of failure.

\section{- Sewer pipe condition assessment practices.}

SPU has used PACP coding on wastewater pipes since 2005. Prior to using PACP coding, a customized coding system, developed in-house, was used since the late 1960s. Seattle Public Utilities primarily implements conventional CCTV inspection methods using a video camera mounted on a remote-controlled tractor unit and considers using a zoom camera on pipes to calibrate various types of maintenance frequencies but not to determine structural conditions. The pipe inspections have been conducted and the GIS has been updated on a regular basis. Inspections have also been prioritized for the last five years using the Sewer Pipe Risk Model. The risk-based strategy identifies pipes that are critical and need to be inspected regularly.

\section{- Sewer pipe repair/rehabilitation/replacement practices.}

Rehabilitation is currently prioritized using an expert system, although SPU is very close to implementing an automated sewer pipe rehabilitation decision model developed by academics and consultants. Currently, CCTV tapes are PACP-coded and a manual decision is made as to whether the pipe should receive no action, a single point repair, a series of point repairs, or a reline. Full pipe replacement is almost never complete since SPU has found this method is not cost effective. 


\section{- Sewer pipe failure prediction.}

Seattle Public Utilities uses predictive failure curves to aid in calculating the risk cost of its sewer pipes. These time-based predictive failure curves use a normalized Weibull based distribution and apply a failure curve to each pipe in the system based on age and material type. Recently, an analysis was conducted to verify the accuracy of SPU's existing curves and to replace these with an expected point failure distribution more precisely based on Seattle's actual sewer pipe failure history.

\section{Orange County, California}

The Orange County Sanitation District (OCSD), formed in 1946, has collected, treated, disposed, and reclaimed the wastewater generated by 2.5 million people in central and northwestern Orange County since its inception. The OCSD includes nine former revenue areas joined to form a single service district, the third largest wastewater agency in the western United States (AWWA, 2005). Additionally, the OCSD operates two treatment plants (located in Fountain Valley and Huntington Beach) and maintains 580 miles of wastewater pipes and 16 pumping stations, of which 250 million gallons of wastewater flows through daily.

Ten million gallons per day of treated wastewater is reclaimed via microfiltration and reverse osmosis. The reclaimed water is used for landscape irrigation and injection into the groundwater sea-level intrusion barrier. Recently, in cooperation with the Orange County Water District, the Ground Water Replenishment System began. Using advanced water treatment facilities, water is purified through microfiltration, reverse osmosis, and ultraviolet disinfection to levels that far exceed drinking water standards (U.S.EPA 2005). In addition, the OCSD has

undertaken management system initiatives in two main areas: Optimized Asset Management and the National Biosolids Partnership Environmental Management System. The OCSD has also engaged in strategic planning activities and has created the Unifying Strategies.

\section{- Condition assessment and rehabilitation practices}

The OCSD adopted an Asset Management Strategic Plan and Framework Analysis in December 2002. This organization developed the first Asset Management Plan in 2005 and the 
second version in 2006, with the goal to create, maintain, and rehabilitate wastewater assets in the most cost effective (lowest life cycle cost) and sustainable manner at the required service level (OCSD, 2006). The basic functional processes of this Asset Management Plan, regarding condition assessment, are to determine current conditions and performance, the asset's likely failure modes, and the probable time of failure. These failure modes include conditions or structural failures, end of useful life, under capacity, not meeting an established level of service, and no longer economic to own and operate.

\section{- Sewer pipe condition assessment practices.}

Closed circuit televising (CCTV) and man entry into manholes are two techniques used to inspect wastewater pipes, in addition to NASSCO standards. Inspections are performed randomly and routinely and random inspections are based on a number of factors including age, $\mathrm{H}_{2} \mathrm{~S}$ levels, CCTV data, Business Risk Exposure, depth of main, land use areas above pipes, and flow rate. Small diameter pipes are monitored randomly through CCTV, based on a historical understanding of the area and, if spills occur, the line is reinspected. Routine inspections are performed for all manholes and large diameter pipes are monitored through CCTV every seven years. Small pipes, with historical root intrusion problems, undergo scheduled root cutting every 18 months.

Wallingford's software system, used for database management, is periodically updated. This system also contains a GIS software system, which is update as soon as information is available. Additionally, NASSCO codes are used with the GIS mapping to represent, visually, pipe conditions, resulting in two separate database systems maintained by the OCSD.

\section{- Sewer pipe repair/rehabilitation/replacement practices.}

Prioritization of repair and rehabilitation is based on structural integrity issues including

life cycle costs, NASSCO codes (high levels), and age of pipes. In addition, compromise of the structural integrity of pipes is based on the rate of corrosion of pipe materials. For example, if more than $25 \%$ of rebar is exposed, due to corrosion, then rebar replacement will occur for that section loss. Business Risk Exposures have also been developed for collection systems to enable the prioritization of asset condition assessments and cleaning. The outputs of this model are used 
to identify potential future Capital Improvement Program projects and may be used in conjunction with capacity modeling, currently being undertaken. These outputs are based on known $\mathrm{H}_{2} \mathrm{~S}$ concentration levels, age, depth, capacity, service area (Urban vs. Metropolitan), and NASSCO standards.

Appropriate management strategies are also allocated based on Business Risk Exposure. Examples of appropriate strategies include

-Schedule regular condition assessments or monitoring.

-CCTV - only clean those pipes that represent the highest risk for both failure modes.

-No CCTV - only assess those pipes in a line that represent significantly different ratings.

-Select a selection from each material type and age whose results can be applied to other assets of a similar material and age.

-Establish work orders for future inspections using the Computerized Maintenance Management System (CMMS).

-Status Quo (Continue Current Strategy).

-Do nothing.

-Renew (Repair/Rehabilitate/Replace).

-Change Maintenance/Operations Levels (e.g., Run to Fail).

-Upgrade (Modify operational functionality - change performance requirements). -Remove (Not used/unnecessary).

\section{- Sewer pipe failure prediction.}

The OCSD utilizes deterioration curves, which are based on age model and $\mathrm{H}_{2} \mathrm{~S}$ categories. The average age of a system is steadily increasing with time. Based on the predicted maximum potential lives of these assets, the maximum life that gravity pipes can reach is 130 years old. In addition, Pomeroy sulfide flux model calculations may be used to help determine corrosion rates and NASSCO standards may be referenced to help determine life expectancy. 


\section{Blacksburg, Virginia}

Blacksburg's Wastewater system is operated by Blacksburg VPI Sanitation Authority Inc. and the Town of Blacksburg. Blacksburg VPI Sanitary Authority was founded in 1962 as a nonprofit organization resulting from the collaborative efforts of the town of Blacksburg and Virginia Tech. The VPI Sanitary Authority owns and operates one treatment plant at Stroubles Creek, consisting of approximately 15 miles of sewer mains and 14 employees. All sanitary and industrial wastewater, treated by the plant, comes from three customers, the town of Blacksburg, Virginia Tech, and part of Montgomery County. Additionally, proportions of the wastewater collected from each customer are calculated based on their tap water usage, which on average consists of $22 \%$, $75 \%$, and $3 \%$ of wastewater traveling to the plant from Virginia Tech, the town of Blacksburg, and Montgomery County, respectively.

The Town of Blacksburg (TOB) owns and operates 21 pumping stations and approximately 145 miles of sanitary sewer, which is divided into 17 basins, called sewer sheds. Additionally, each sewer shed is further divided into sub-sewer sheds.

\section{- Condition assessment and rehabilitation practices}

The town of Blacksburg prioritizes repair, rehabilitation, and replacement of the wastewater collection system based on expert decisions considering the following criteria, (a) age of the pipe, (b) Inflow \& Infiltration problem, (c) surge problem, (d) immediate attention requirements. The TOB has experienced overflows and surcharges in its wastewater collection system primarily during wet weather events. Due to these overflows and surcharges, Blacksburg has violated the Clean Water Act and recently contacted local consultants to conduct a sanitary sewer study.

Objectives of this study included the following:

- Assess the capacity of the existing wastewater collection system to determine if, when, and where surcharging or overflows occur. 
- Assess the overall condition of the existing wastewater collection system to determine if any portion of the system needed repair, replacement, or rehabilitation.

- Assess the status of the wastewater collection system to determine if the system is in compliance with current and pending regulatory requirements (Wiley \& Wilson, 2006).

Three aspects of this study including capacity, condition and current and pending regulations as discussed below.

Capacity. The assessment of the existing wastewater collection system's capacity allowed the town to determine where capacity problems currently and potentially exist. The two capacity issues assessed included overflows and surcharging. Overflows may be determined by visual inspection, while surcharging can be detected by manhole inspections and flow meter measurements.

Condition. Cracked and broken pipes, leaking pipe joints, sags in the pipes, cracks and holes in the manhole walls, leakage in the manhole joints, and leakage through the frames to manhole joints were problems found in poor collection systems that caused inflow and infiltration and reduced the capacity of sewer lines. In addition, some of these condition defects reduced the capacity of the system from a structural standpoint. For example, solid build up in sags and partial flow blockages in broken pipes reduced the cross sectional areas of the pipes and; therefore, decreased the pipes' capacities.

Current and pending regulations. This study in Blacksburg involved research of the current and pending state and federal regulations applicable to wastewater collection systems. The historical data, including previous studies, was reviewed in order to identify the problem areas for rehabilitation decisions. 


\subsection{Software Programs}

Listed included many developed programs ranging in degree of sophistication. While many of these programs were developed as planning models, all are focused on evaluating the life of an asset. "Protocols for Assessing Condition and Performance of Water and Wastewater Assets Assessment" (Urquhart, 2000) offers an extensive list that summarizes the Condition and Network Assessment Programs for wastewater, which has been developed worldwide. Table 2.2 is based on this work. Note: This list is by no means comprehensive and there are a number of other programs and developments currently underway. 
Table 2.2 Condition Assessments and Planning Technique

\begin{tabular}{|c|c|c|c|c|c|c|}
\hline Technique & Focus & Data needs & Commercialized & Integration & $\begin{array}{l}\text { Skills } \\
\text { Required }\end{array}$ & $\begin{array}{l}\text { Degree of } \\
\text { sophistication }\end{array}$ \\
\hline $\begin{array}{l}\text { AQUA- } \\
\text { WertMin }\end{array}$ & $\begin{array}{l}\text { Planning of } \\
\text { CCTV } \\
\text { inspection, } \\
\text { rehabilitation, } \\
\text { and } \\
\text { construction } \\
\text { for sewer } \\
\text { networks }\end{array}$ & $\begin{array}{l}\text { Requires } \\
\text { CCTV data }\end{array}$ & $\begin{array}{l}\text { Yes Available } \\
\text { from Germany, } \\
\text { limited } \\
\text { application }\end{array}$ & No & $\begin{array}{l}\text { High- asset } \\
\text { manager or } \\
\text { engineer }\end{array}$ & Moderate \\
\hline CARE-S & $\begin{array}{l}\text { Service levels, } \\
\text { budget setting, } \\
\text { life cycle cost } \\
\text { and } \\
\text { rehabilitation } \\
\text { planning }\end{array}$ & $\begin{array}{l}\text { Dependent on } \\
\text { models applied }\end{array}$ & $\begin{array}{l}\text { No - research } \\
\text { applications only }\end{array}$ & $\begin{array}{l}\text { No- } \\
\text { standalone } \\
\text { tool }\end{array}$ & $\begin{array}{l}\text { High - } \\
\text { professional } \\
\text { engineering } \\
\text { skills }\end{array}$ & $\begin{array}{l}\text { Basic-generic } \\
\text { approach }\end{array}$ \\
\hline KureCAD & $\begin{array}{l}\text { Applies GIS } \\
\text { analysis for } \\
\text { prioritization } \\
\text { of sewer } \\
\text { rehabilitation }\end{array}$ & $\begin{array}{l}\text { High - good } \\
\text { quality asset } \\
\text { and failure } \\
\text { data }\end{array}$ & $\begin{array}{l}\text { Yes - used by a } \\
\text { number of } \\
\text { Australian } \\
\text { utilities }\end{array}$ & $\begin{array}{l}\text { Yes - } \\
\text { integrates } \\
\text { with } \\
\text { relational } \\
\text { databases }\end{array}$ & $\begin{array}{l}\text { High - asset } \\
\text { manager or } \\
\text { engineer }\end{array}$ & High \\
\hline AQUA-Selekt & $\begin{array}{l}\text { Sewer } \\
\text { condition } \\
\text { forecast }\end{array}$ & $\begin{array}{l}\text { High - CCTV } \\
\text { inspection data }\end{array}$ & $\begin{array}{l}\text { Yes - has had } \\
\text { limited } \\
\text { application in } \\
\text { Europe }\end{array}$ & None & $\begin{array}{l}\text { High- } \\
\text { professional } \\
\text { engineering } \\
\text { skills }\end{array}$ & High \\
\hline SCRAPS & $\begin{array}{l}\text { Expert systems } \\
\text { that prioritizes } \\
\text { sewer } \\
\text { inspections }\end{array}$ & $\begin{array}{l}\text { Information on } \\
\text { critical assets }\end{array}$ & $\begin{array}{l}\text { Yes - available } \\
\text { from WERF }\end{array}$ & No & $\begin{array}{l}\text { High - asset } \\
\text { manager }\end{array}$ & Moderate \\
\hline $\begin{array}{l}\text { WRc sewer } \\
\text { rehabilitation } \\
\text { manual }\end{array}$ & $\begin{array}{l}\text { Framework for } \\
\text { assessing the } \\
\text { condition and } \\
\text { performance of } \\
\text { sewerage } \\
\text { networks, risk } \\
\text { of failure }\end{array}$ & $\begin{array}{l}\text { High - but can } \\
\text { be customized } \\
\text { to be } \\
\text { affordable }\end{array}$ & $\begin{array}{l}\text { Framework } \\
\text { available as } \\
\text { manual }\end{array}$ & $\mathrm{NA}$ & $\begin{array}{l}\text { High- } \\
\text { professional } \\
\text { engineering } \\
\text { skills }\end{array}$ & $\begin{array}{l}\text { Basic-generic } \\
\text { approach }\end{array}$ \\
\hline
\end{tabular}




\section{CHAPTER 3}

\section{Wastewater Pipe FaIlure ModeS AND MeChanisms}

In this chapter, description of life cycle and of failure modes and mechanisms of wastewater pipe based on material are presented.

\subsection{Pipe Life Cycle}

The material system life cycle consists of the system's design, development, construction, operation and maintenance, and repair/rehabilitation/replacement. Technical systems and manmade products such as the infrastructure system and the pipes may be viewed as having a particular life cycle. These systems or products can be considered as some sort of living system of organisms since they come to being, grow and interact with their environment, age, and eventually die. All repairs, rehabilitations, and renewals can be considered external influences that affect the life cycle of the system and products. These living systems or organisms share a common behavior-an idea often referred to as the bathtub theory. The bathtub theory is a function of the probability of failure over time. The term "bathtub" comes from the line commonly produced by the probability curve (Farshad, 2006). A representation of the bathtub theory as related to the failure probability of piping systems can be seen in Figure 3.1. 


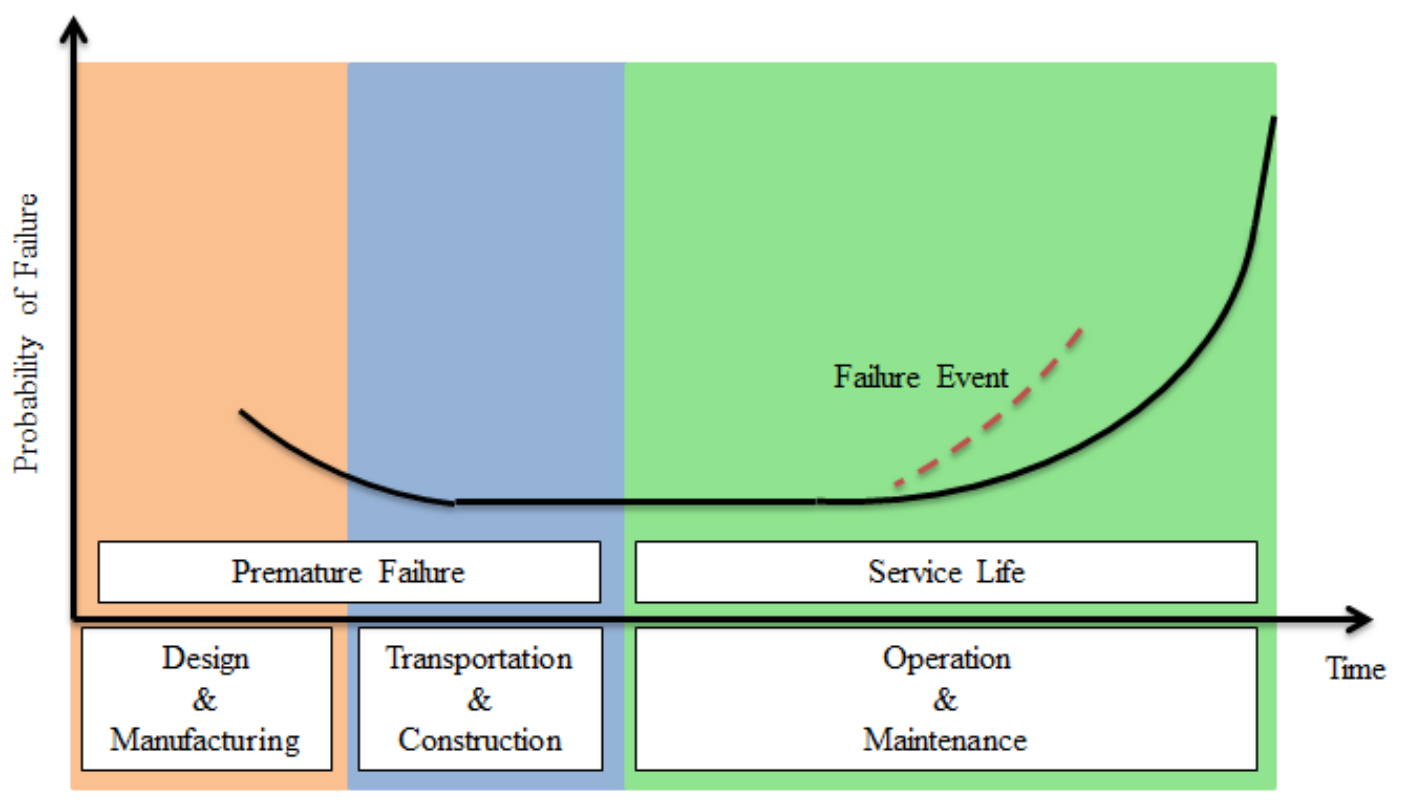

Figure 3.1 Bathtub Theory

According to the bathtub theory, wastewater pipe may not have $100 \%$ quality when installed in the ground. Some defects and damage may have occurred during the design and manufacturing processes, thereby lowering the quality of the overall product. Some causes of failure that can occur during the design and manufacturing stages are poor design, poor project planning, dimensioning, observation and quality control, manufacturing defects, and storage. The construction process may also have a permanent effect on pipe failure, including failures due to transit, human error, and poor workmanship. Careless or improper construction processes may also reduce the performance of pipe. Throughout years of service, operation and maintenance also affect the pipe performance through various failure causes such as mechanical, thermal, chemical, biological, external interferences, natural catastrophes, and inappropriate services and maintenance. We have identified failure causes based on pipe life cycle of all pipe material and Table 3-1 shows the life cycle of concrete pipe (Najafi, 2005; NASSCO, 2003; DIPRA, 2003; NRC-CNRC, 2003; Garcia, 2002; Davies, 1999; Serpente, 1993; Burn, 2005; Kellagher, 2002; Jason Consultants, 2007; Makar, 2000; Moser, 2001). Pipe life cycle for all pipe material can be found in Appendix A. 
Table 3.1 Concrete Wastewater Pipe Life Cycle

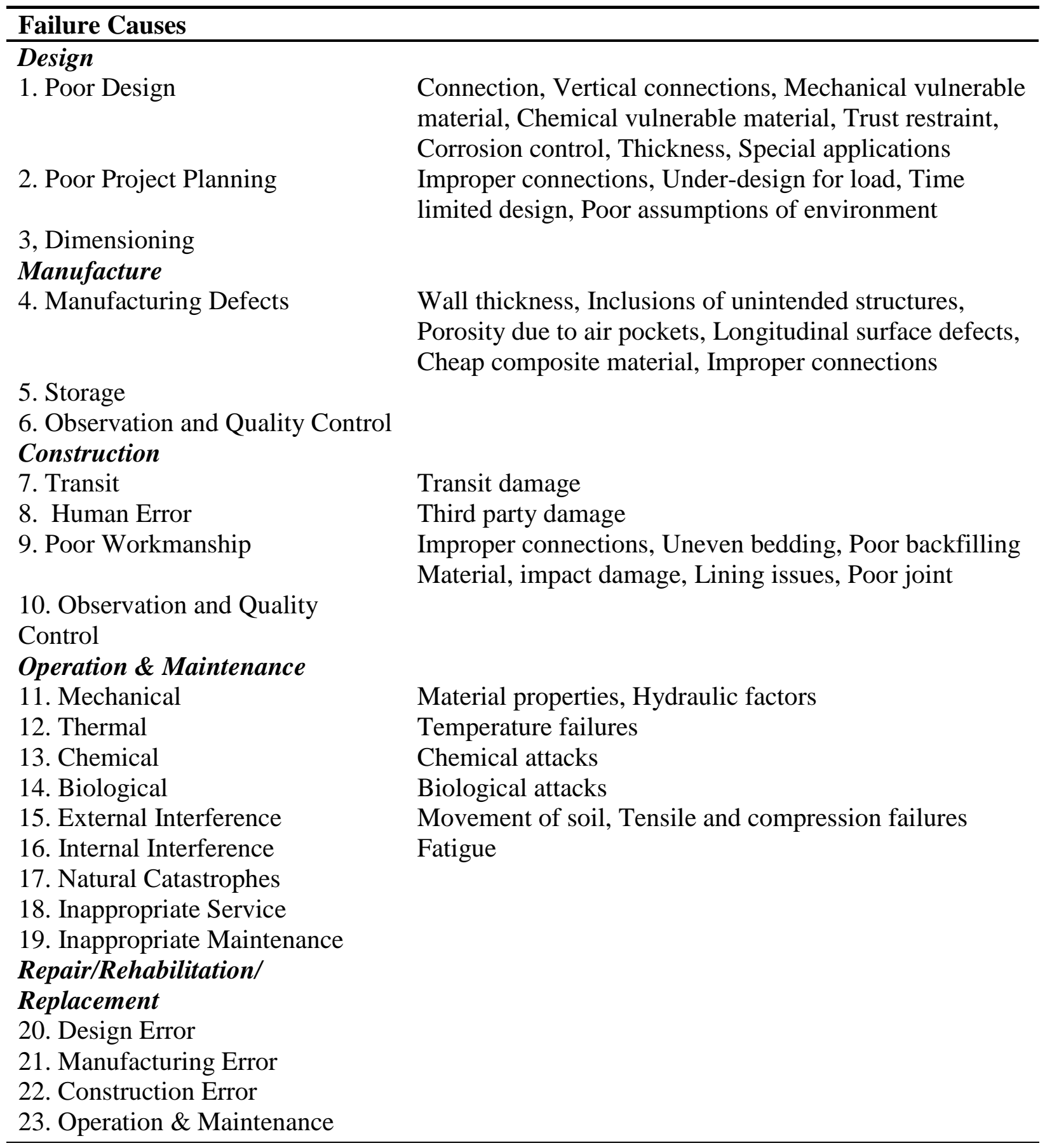




\subsection{Failure Modes and Mechanisms}

The failure processes in buried pipes are much more complex than expected. At the most basic level, pipe failures are caused by applied forces exceeding the residual strength of the pipe material. In general, the forces applied to buried pipe can be categorized into five groups: those produced by internal pressure, bending forces, crushing forces, soil movement-induced tensile forces, and temperature-induced expansive forces. The proper repairs of failures depend on knowing the causes and selecting the appropriate repair procedures that take these causes into account; otherwise, the repair may only be temporary. The proposed study in this phase examines the durability and performance of buried pipes to help those in the utilities field to understand the effects of various factors as well as determine the best pipe material for specific site conditions.

The failure mode of wastewater pipes can be defined as a type of failure that occurs to the pipe. Meanwhile, the failure mechanism is an event that causes the pipe to reach one of the combined strength and serviceability limit states (Farshad, 2006). Limit states can be defined as either an ultimate limit state or a serviceability limit state. The ultimate limit state defines a condition at which the strength of the pipe is reached. Examples of this state may be the loss of water tightness, bursts, and loss of stiffness. The serviceability limit state defines a condition at which a particular function of the pipe is no longer fulfilled. Examples of this state may be large deformations, change of color, buckling, clogging, abrasions, and local damages (Farshad, 2006).

We have prepared various modes and mechanisms of pipe failure by material type based on extensive literature reviews and pipe association interviews. Table 3.2 presents main line and joint failure modes of concrete pipe (Najafi, 2005; NASSCO, 2003; DIPRA, 2003; NRC-CNRC, 2003; Garcia, 2002, Davies, 1999; Serpente, 1993; Burn, 2005; Kellagher, 2002; Jason Consultants, 2007; Makar, 2000; Moser, 2001). The failure modes and mechanisms are classified into two groups: main pipe and joint. All failure modes are listed and separated into a more specific failure type. For example, the failure mode "crack" can be separated into longitudinal crack, circumferential crack, spiral crack, and multiple cracks based on the material type. A complete list of failure modes for all pipe matrial is provided in Appendix B. 
Table 3.2 Failure Modes of Concrete Wastewater Pipe

\begin{tabular}{|c|c|c|}
\hline \multicolumn{3}{|c|}{ Concrete Wastewater Pipes Failure Modes } \\
\hline \multicolumn{3}{|r|}{ A. Main Line } \\
\hline \multicolumn{3}{|c|}{ Typical Failure Mode } \\
\hline \multicolumn{3}{|c|}{ Structural Failure } \\
\hline 1 & Crack & Longitudinal crack, Circumferential crack, Multiple cracks \\
\hline 2 & Fracture & $\begin{array}{l}\text { Longitudinal fracture, Circumferential fracture, Multiple } \\
\text { fractures }\end{array}$ \\
\hline 3 & Broken & \\
\hline 4 & Hole & \\
\hline 5 & Deformed & Vertical deformed, Horizontal deformed \\
\hline 6 & Collapsed & \\
\hline 7 & Surface damage & $\begin{array}{l}\text { Roughness increased, Aggregate visible, Aggregate projecting, } \\
\text { Aggregate missing, Missing wall, Surface spalling }\end{array}$ \\
\hline 8 & Lining Failure & $\begin{array}{l}\text { Defective Weld, Detached, Defective end, Blistered, Service } \\
\text { cut shifted, Abandoned connection, Overcut service, Undercut } \\
\text { service, Buckled, Wrinkled }\end{array}$ \\
\hline \multicolumn{3}{|c|}{ Operational and Maintenance Failure } \\
\hline 9 & Deposits & Attached, Settled, Ingress \\
\hline 10 & Roots & Fine, Tap, Medium, Ball \\
\hline 11 & Infiltration & Weeper, Dripper, Runner, Gusher \\
\hline 12 & Exfiltration & \\
\hline 13 & Obstacles/Obstructions & $\begin{array}{l}\text { Pipe material in invert, Object intruding through wall, Object } \\
\text { wedged in the joint, Object through connection, External pipe } \\
\text { or cable, Construction debris, Rocks, Other obstacles }\end{array}$ \\
\hline \multicolumn{3}{|c|}{$\begin{array}{l}\text { Rare Failure Mode } \\
\text { Structural Failure }\end{array}$} \\
\hline 14 & Crack & Spiral crack \\
\hline 15 & Fracture & Spiral fracture \\
\hline & & B. Joint \\
\hline \multicolumn{3}{|c|}{ Typical Failure Mode } \\
\hline 1 & Lined Joint & Separated Joint \\
\hline 2 & Rubber Gasket Joint & $\begin{array}{l}\text { Corrosion degradation of reinforcing wires, Gasket degradation, } \\
\text { Concrete degradation, Mortar seal degradation, Seal } \\
\text { displacement }\end{array}$ \\
\hline 3 & Steel End Ring Joint & Weld failure, Corrosion of spigot, Ovality of pipe \\
\hline
\end{tabular}




\section{Concrete Wastewater Pipe}

Most of the time, the failure of concrete pipe occurs as a result of deflection and cracking related to excessive external loading and corrosion. Initial failure is possible and usually caused by design error or impacts during construction. Corrosion leads to structural failure. Sulfuric acid that corrodes the pipe near the crown is formed by the hydrogen sulfide gas $\left(\mathrm{H}_{2} \mathrm{~S}\right)$ in sewage and condensing fluid (Garcia, 2002). Pre-stressed concrete cylinder pipe (PCCP) is constructed by circumferentially winding a high strength steel wire, under tension, around a concrete core containing a sheet steel cylinder, which serves as a watertight membrane. Typically, the ability of the steel pre-stressing wire determines the service life of such pipe (Rothman, 1986). Concrete is a mineral-based material. Thus, softening of the pipes can occur through lime leaching when certain soil and water types are contacted. The process also involves corrosion in the reinforcing wire with a corresponding loss of the structural integrity as the physical strength of the wire degrades. The reinforcing/pre-stressing wire in RCP/PCCP pipe is susceptible to stray current corrosion if the cathodic protection or other corrosion-resistant components are not present. If the pre-stressing wire corrodes and swells, the mortar may crack, which can lead to the catastrophic failure of the pipe wall structure (Reed, 2004).

\section{Ductile Iron (DI) Wastewater Pipe}

In most cases, the factor that leads to failure in ductile iron wastewater pipe is corrosion, which occurs when a pipeline is exposed to corrosive substances. Corrosion can be either internal or external. Internal corrosion is caused by exposure to highly corrosive substances in the wastewater from industrial sources. Domestic corrosive wastewater by itself is rarely strong enough to cause corrosion. In gravity pipelines, slime build up can ultimately result in corrosion, particularly if the wastewater flow is very slow (velocity less than $2 \mathrm{ft} / \mathrm{s}$ ). Compared to water pipes, wastewater force main pipelines are less likely to have induced strain from operation pressure due to the lower pressure used in the system.

Meanwhile, external corrosion occurs when the pipeline is subjected to a corrosive environment. The corrosion rate can be determined by the characteristics of the surrounding soil. Resistivity, Redox potential, and $\mathrm{pH}$ level are believed to be the predominant factors. Such 
corrosion usually results in a localized attack and the formation of corrosion pits (Reed, 2004). These pitting holes are unlikely to cause pipe structural failure; however, they lead to leakage, infiltration, and exfiltration.

Designs for corrosion protection are very important in ductile iron pipe. Internal pipe is typically protected from corrosion by cement mortar. External pipe is commonly shielded by a factory zinc coating. Another issue in corrosion deterioration is stray currents, which are direct currents flowing through the earth that impact underground piping. When stray direct currents accumulate on a metallic pipeline, they can induce electrolytic corrosion of the metal or alloy (Bond, 1997). Thus, stray current protection is recommended if the pipeline is located close to the sources of stray current, which include cathodic protection systems, direct current power trains or street cars, arc-welding equipment, direct current transmission systems, and electrical grounding systems.

\section{Cast Iron (CI) Wastewater Pipe}

Cast iron is made by adding larger amounts of carbon to molten iron than the amount used to make steel. Cast irons typically have 2.5 to 4 percent of carbon (by weight) whereas steels have less than 1.2 percent by weight. Adding more carbon to molten iron makes it more fluid and easier to cast; however, when the metal solidifies, some or most of the carbon may form graphite flakes (Makar, 2000; NRC-CNRC, 2009). Cast irons can be manufactured using two methods. Older pipes were made of pit casting, which is a static process, whereas newer pipes are made of centrifugally spun casting. Cast iron is fundamentally brittle material that has less yield strength than ductile iron.

The main categories of failure that occur in cast iron pipes are cracks leading to fractures and collapses caused by internal or external loading. The predominant pipe stresses in smaller pipe (less than 12 inches in diameter) are due to externally induced bending loads that can cause failure in such pipe. In larger pipe, crushing failures predominate, leading to longitudinal factures (Reed, 2004). Losses in pipe wall thickness resulting from corrosion combined with the external 
loads can initiate failure as well. The two main forms of corrosion are general corrosion and localized pitting corrosion. The presence of pitting corrosion can form pitting holes.

\section{Polyvinyl Chloride (PVC) Wastewater Pipe}

Polyvinyl Chloride (PVC) pipes are made of viscous-elastic material. Creep and stress relaxation are considered in this material type. Generally, PVC pipes are stronger in circumferential direction than longitudinal direction due to the oriented structure of the pipe material. Although longitudinally oriented PVC pipe exists, it is rare. The axial over deflection induces bending stress along the pipe and eventually causes cracking. PVC pipe failure also can be caused by cyclic fatigues. For example, cyclic loading from turning pumps on and off can lead to premature failure in PVC pipe. During construction or transit, accidents may cut or damage the pipe surfaces both internally and externally. Poor installation and workmanship may also result in damage to the pipe. For example, over-insertion may crack the end of the pipe.

\section{Polyethylene (PE) and High Density Polyethylene (HDPE) Wastewater Pipes}

Polyethylene (PE) and High Density Polyethylene (HDPE) are viscous-elastic materials. Creep and stress relaxation should be considered in these material types. Various factors affecting PE pipe include but are not limited to chemical attack, loading, temperature, and installation practices. Three basic modes of PE pipe failure are recognized as ductile failure at relatively high stresses, brittle fracture at intermediate stress levels, and environmental stress cracking or stress corrosion cracking at low stress levels (Wienhold, 2006).

\section{Clay Wastewater Pipe}

Clay or vitrified clay pipes are strong and chemical resistant because of their ceramic material properties. Crushing failure rarely occurs in new clay pipe, although clay pipes are brittle. The properties of the clay pipe do not change over time, so age of the pipe does not affect the durability of the pipe. The typical structural failure mode of the clay pipe is cracks. 
Failure in clay pipes is caused by three factors: loading, bedding system, and foundation. Most failures resulting from construction, bedding, or foundation become evident in the first two years as soil consolidates completely. During construction, it is very important that the bell holes be dug properly or else the pipe bell may crack. Localized failure is possible as a result of inadequate longitudinal support, poor bedding, or over-compacting. Excessive point loading can also create a star break in clay pipe. Impact failure resulting from third-party or over-compacting damage is possible as well. Poor construction may lead to lateral shear, and differential settlement may cause shear between the manhole and the pipe. Joints are the key weakness in clay pipes. Parallel offsets, pulled joints, excessive angular deflection, and leakage are the main joint failure modes of clay pipes. These joint failures may lead to root intrusions and I\&I, which eventually affect the pipe's hydraulic capacity and functionality.

\section{Brick Sewer}

Failure modes of brick sewer consist of mortar loss due to abrasion, poor cleaning practice, chemical attack, cracking due to excessive external loading, and differential settlement. Mortar failure will eventually lead to infiltration and exfiltration to/from the environment, carrying soil particles into the sewer and leading to a loss of bedding support (Garcia, 2002). Other failures include root intrusion, sediment blocking the flow, and object intrusion.

Failure modes and mechanisms of various pipe material were discussed. Typically, structural failures of wastewater pipe occur due to deflection and cracking related to excessive external loading that resulting in cracks, fractures, or broken. Some failures are distinctive to certain materials as presented. Typical operational and maintenance failures are blockages, root intrusions, and inflow \& infiltration, for example. With the understanding of the failure modes and mechanisms, the performance index can be developed. 


\section{CHAPTER 4}

\section{Development of a Wastewater Pipe Performance INDEX}

Condition/Performance assessment and deterioration modeling are rapidly becoming an indispensable part of life cycle and asset management activities. Developing an efficient approach for evaluating the condition and performance of wastewater pipes can provide decision-making tools that are required to deal with large volumes of deteriorating, buried pipelines in a system.

Many utilities in the U.S. use the Water Research Center's (WRc's) and the National Association of Sewer Service Companies' (NASSCO's) defect rating systems, although some utilities use their own, in-house products; however, most of these rating systems are based on the closed circuit television system (CCTV) inspection of wastewater pipes. A robust performance index that considers both the inspection data and other important data, such as soil characteristics, loading, and location, is required to obtain better evaluations of the conditions and performance of wastewater pipes. Data are needed for use in assessing the pipes' conditions and performance. Some parameters are more significant than others, so a complete data structure would enable the utility to collect the appropriate and required data. Close examination and careful identification of performance parameters are required for the development of an accurate performance index and 
an accurate prediction model for wastewater pipes. In addition, no standard performanceevaluation tool is available that considers the roles of different factors, such as pipes' characteristics and internal and external environmental factors. Currently, many utilities assess the conditions and performance of wastewater pipes in their own manner based on their experience. This method may provide correct results, but a more standardized tool should be developed and used to ensure that the assessment process produces reliable and accurate information concerning the condition of the pipes.

We investigated the life cycle of wastewater pipes and identified the causes of failures in different phases, including design, manufacture, construction, operation maintenance, and repair/rehabilitation/replacement as discussed in the previous chapter. We assessed the various modes and mechanisms of the failures that occur in the pipes that make up wastewater infrastructure systems. Different pipe materials have different failure modes and mechanisms. This model was designed based on the available information related to the life cycle, failure modes, and mechanisms of wastewater pipes.

A performance index for wastewater pipes was developed using two mathematical methods, weighting factors, and a fuzzy inference system. The performance index considers conditions (defects) identified from inspections, e.g., cracks, holes, and corrosion, as well as other parameters that affect the conditions and performance of wastewater pipes. The performanceindex model consists of parameters from a wastewater pipe system, including the physical/structural, operational/functional, environmental, and other parameters of the pipes as well as the entire system. Structural parameters included the slope of the pipe, its age, and the material of construction, and environmental parameters included the properties of the soil and the external loading on the pipe.

\subsection{Methodology of the Pipeline Performance Index}

This section provides an overview of the weighted factor and fuzzy inference system theories used in the performance models. 


\section{Weighted factor}

Parameters (including pipe condition from inspection data) must be rated and assigned scores before putting into equation. Combining defects score to a condition scale can be challenging. Typical methods that utilities use currently can be summarized into three categories, subjective grading, distress-based evaluation, and non-destructive evaluation (Mehle et al, 2001).

A typical sewer defect score is defined by a number from 1 to 5 . It is determined by a defect score calculation that based on the summation of the deduct values for different defects in the pipe segment. Other parameters are graded by the level of performance. For example, if flow velocity is lower than a certain level, problems such as internal corrosion may occur. However, the weighted factor method assumes that each parameter is independent, which is not the case in the real situations. In addition, assigning weights to parameters proves to be very difficult and required extensive studies and research throughout the subject (Rahman et al, 2004).

The weighted factor methods calculates the index by assigning a weight and score to each factor. The weighted factor equation is presented in equation 4-1.

$$
Y_{\mathrm{i}}=\sum w_{i} \cdot X_{\mathrm{i}}
$$

Where;

$$
\begin{aligned}
& w_{\mathrm{i}}=: \text { weighted factor } \\
& X_{i}=: \text { parameters } \\
& Y_{i}=: \text { index }
\end{aligned}
$$

\section{Fuzzy Inference System}

Fuzzy logic algorithms were introduced by Zadeh in 1965 for complex systems and decision processes (Zadeh, 1965). Fuzzy logic was developed to assist the classical set (binary condition, zero or one). A fuzzy set is a set without a clearly defined boundary. Fuzzy inference is the process of formulating the mapping input to an output using fuzzy logic proposed by Mamdani in 1975. It has found application in a number of areas of infrastructure management, such as bridges, highways, oil and gas pipeline, and water pipe networks. Mamdani's fuzzy 
inference method is one of the most commonly used fuzzy methods and has widespread acceptance. The two main fuzzy inference methods are the Mamdani and Sugeno. The Mamdani's method was proposed in 1975, whereas the Sugeno's method was introduced in 1985 (Mamdani, 1975; Sugeno, 1985). The difference between the two methods is that, the output functions used in Mamdani method are fuzzy sets while, in Sugeno method are linear or constant (Sivanandam, et al, 2007). Example of Mamdani's fuzzy inference system is shown in Figure 4.1.

In this research, the Mamdani's method was selected since the output fuzzy sets can account for imprecision and uncertainty. Fuzzy systems are structured numerical estimators. They start from highly formalized insights about the structure of categories found in the real world and then articulate fuzzy IF-THEN rules as expert knowledge. Fuzzy systems combine fuzzy sets with fuzzy rules to produce an overall complex nonlinear behavior. Fuzzy sets are opposite of classic, or crisp sets. In terms, the set contains varying degrees of membership within a set where a classic set has a specific membership which is defined. Challenges exist in constructing fuzzy rule, selecting membership function, and deciding the defuzzification process.

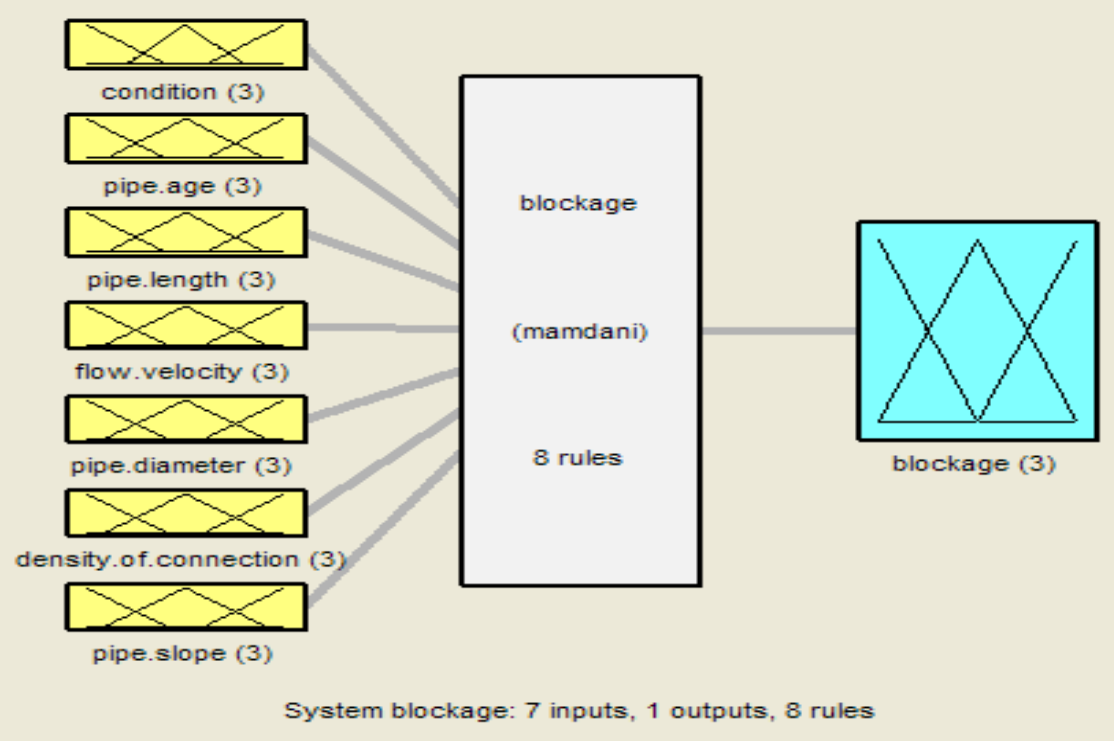

Figure 4.1 Fuzzy Inference System 
The fuzzy inference system is made up of series of rules and data. Such rules implied into the program may be written as "IF" and "THEN" statement. "IF" corresponds to the data implies these certain conditions "THEN" perform these actions. Two main concepts govern fuzzy inference modeling are fuzzification and defuzzification (Shamir, 1979). Fuzzification means separating a range of input variables into a continuum of scalar, or fuzzy, grades. For example, a range of temperatures can be classified into three grades; low, medium, or high. Defuzzification on the other hand takes the fuzzy conclusion and computes it into a single variable corresponding to the grades. The defuzzification process is much more complex than fuzzification. The process includes deciding how to modify the membership function since each value has a different grade of membership. Each membership function must then be transferred into a single membership function, and finally a single value is output. Some methods used for calculating this final defuzzified number are the average maximum method, the weighted average maxima method, and the centroid method which is most common.

There are several limitations which should be stated when constructing a fuzzy inference system. One limitation is that an engineer must be knowledgeable in various computer languages and know the basics of constructing a fuzzy inference system. Once the fuzzy system is written, there will also be the tasks of handling of debugging, calibrating, and validating the expert system. Maximum of parameters that can be used in each fuzzy inference system is limited for a system to be understandable and transparent.

\subsection{Model Development}

All parameters for a wastewater pipe system, including physical/structural, operational/functional, environmental, and other parameters, were included in developing a performance index for wastewater pipes. Some parameters that could have affected wastewater pipes may be missing, but we had summarized most of the essential parameters based on literature review, interviews with utility and pipe association representatives, and questionnaires. The parameters that were used in the model were grouped into pipe characteristics, pipe conditions (structure), internal environment, and external environment. The system was used to evaluate each parameter and to combine them mathematically through a weighted summation and a fuzzy 
interference system that reflected the relative importance of the various factors. The performance model was designed to analyze concrete, clay, and PVC pipes since they account for the majority of wastewater pipes installed in the U.S.

\subsection{Model Layout}

The model rates wastewater pipe performances in eight modules, including integrity, external corrosion, internal corrosion, surface wear, blockage, in/exfiltration, root intrusion, and capacity modules. Figure 4.2 illustrates the schematic diagram of the performance index model.

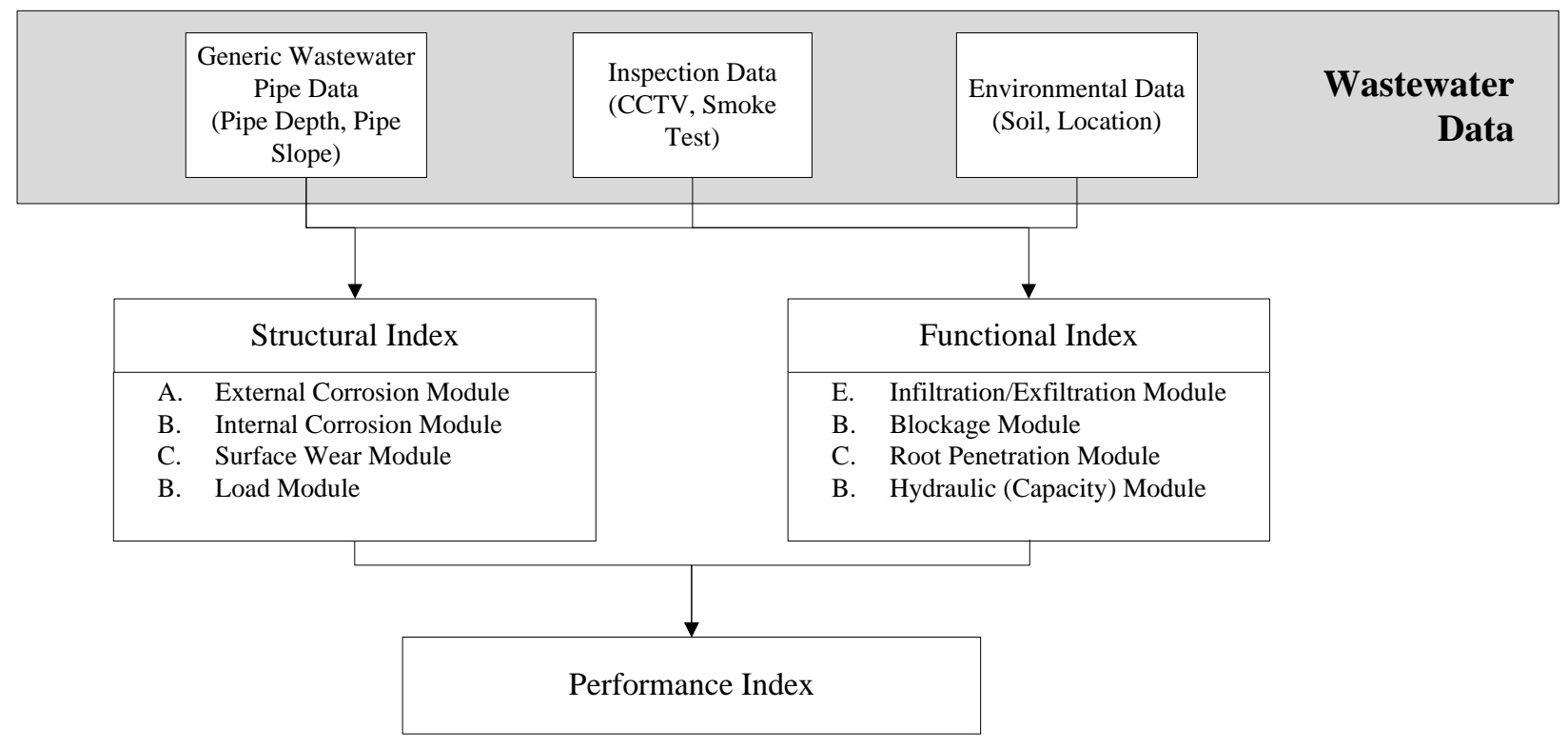

Figure 4.2 Performance Index Scheme

A maximum index of each group shows a potential failure path of a particular pipe and eventually becomes Structural /Functional index. Finally, the structural and functional index are combined into an overall performance index. In the model, each index rates wastewater pipes in scale from zero to five in which five indicates the best condition. Pipe defects from inspections are rated according to the PACP standard. 


\subsection{Data Structure}

We identified close to 100 possible parameters affecting wastewater pipe infrastructures. The lists of parameters were sent to participating utilities within and outside the United States in order to gather feedback for improving the data structures. Based on the feedback received, changes and updates to the list were made. The goal is to eventually create a standard data structure for wastewater pipe infrastructure. In addition to developing an inclusive list of all parameters that can ultimately affect the deterioration of the pipe infrastructure, a supplemental goal is to generate a detail methods and protocols for data collection.

The standard data structure was developed to aid the decision-making process in asset management program. In addition, the data structure can be used to develop a condition index, prediction model, prioritization for repair and rehabilitation, prioritizing inspection, operation and maintenance plans, a capital improvement program, and high-level decision-making processes. The parameters were divided into five classes based on their characteristics: Physical/Structural, Operational/Functional, Environmental, Financial, and Others (see Figure 4.3).

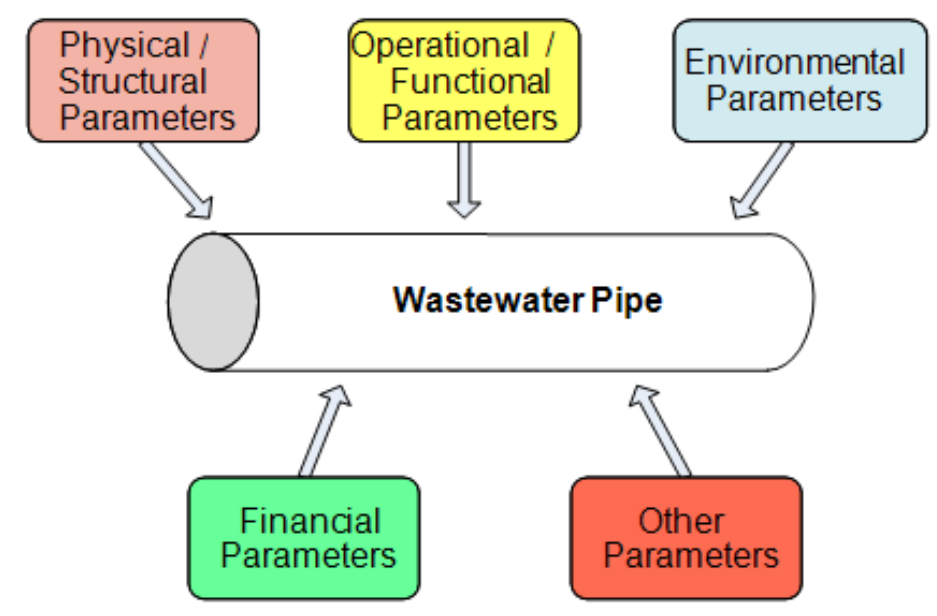

Figure 4.3 Classification of Pipe Parameters

In this study, we considered data from major cities as well as smaller towns within the United States. Due to utilities' lack of readily available data on the number of parameters, the data structure was broken into four separate standards: wood, bronze, silver, and gold. 
Wood standard $(\mathbf{W})$ is for utilities that are very small and do not have sufficient resources to collect a large amount of data or utilities establishing a wastewater pipe database. This standard represents the six essential and five preferable parameters for a wastewater pipe based on the knowledge of pipe infrastructure.

Bronze standard (B) is for utilities that are small and do not have a lot of man hours due to a small workforce. This standard represents the 11 essential and 12 preferable parameters for a wastewater pipe.

Silver standard (S) is for utilities that are larger than bronze utilities, but still may not have a special team designed to devote all their time to developing wastewater pipe data parameters within a system. This standard represents the 23 essential and 28 preferable parameters for a wastewater pipe.

Gold standard (G) is for utilities representing some of the largest cities within the United States that have a special team devoted to the continual updating of the wastewater database. This standard entails 51 essential and 47 preferable parameters affecting the wastewater pipe. Ultimately, utilities may not have resources and/or time to collect all these data; however, some of the data may already be available within city databases while other data can be acquired from other sources, such as soil data from the Soil Survey Geographic (SSURGO) Database. Table 4.1 shows standard wastewater pipe data structures, including wood, bronze, silver, and gold standards (Heastad, 2004; FCM and NRC, 2004; NCPI, 2004; Najafi, 2005; CARE-S, 2002; NASSCO, 2003; NRC-CNRC, 2003; Makar, 2000; Rajani, 2000; 2002c). A list of essential and preferable parameters of gold standard wastewater data structure with description is presented in Appendix C. 
Table 4.1 Wastewater Pipe Data Structure

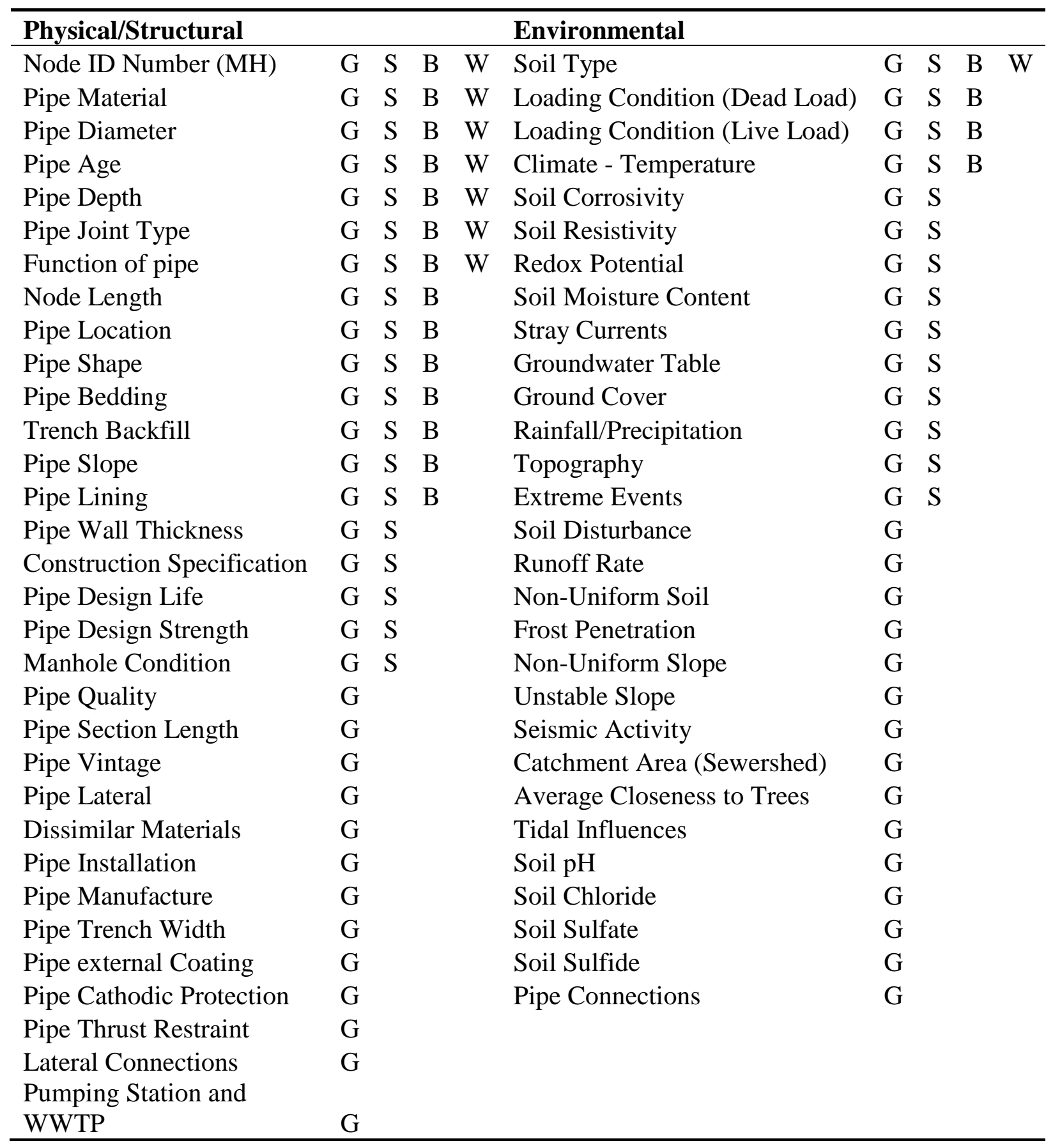


Table 4.1 Wastewater Pipe Data Structure cont

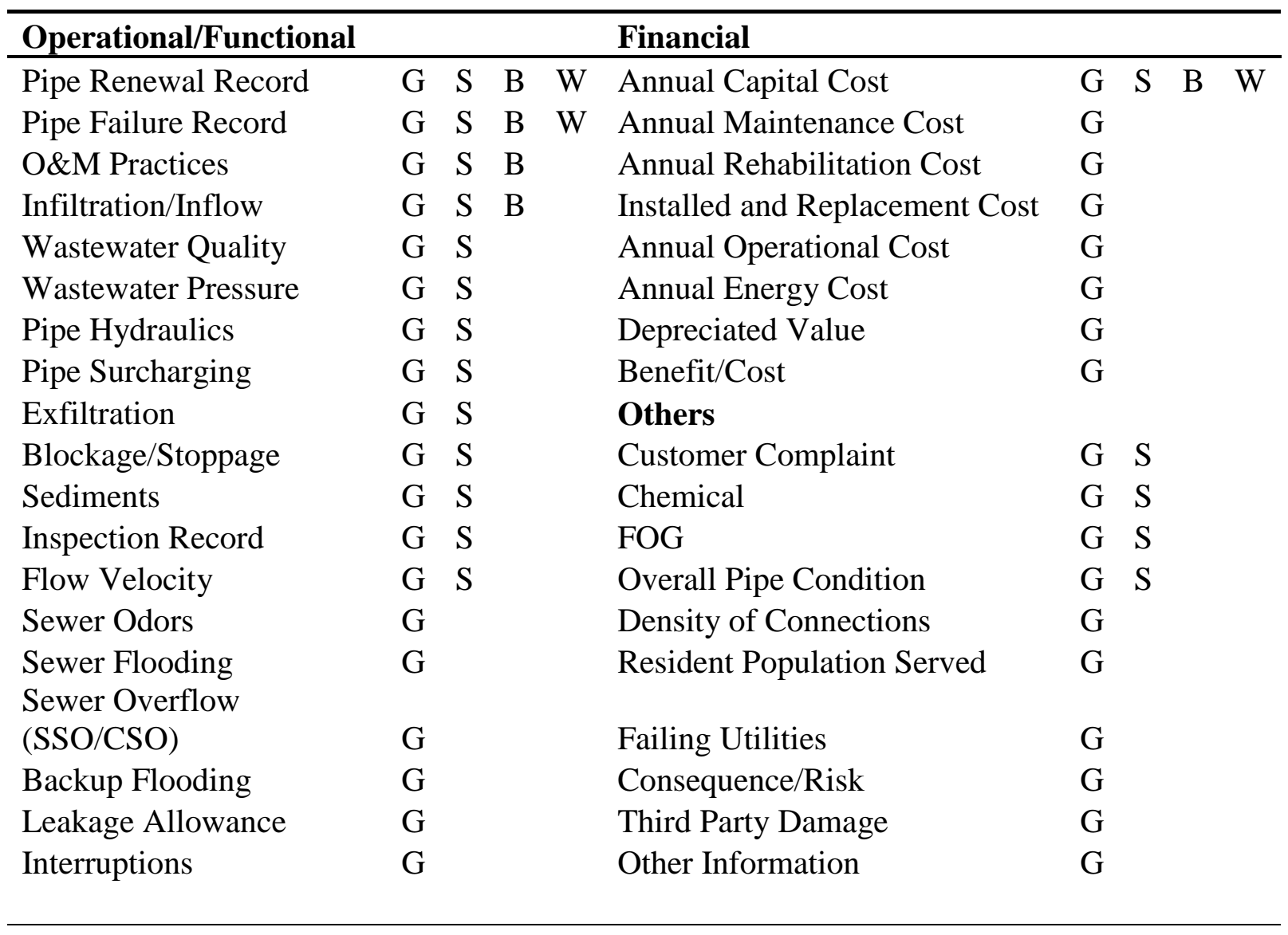

Note: G= Gold Standard, S= Silver Standard, B= Brown Standard, W= Wood Standard

\subsection{Data Collection Methods and Protocols}

Wastewater pipe infrastructure data has been previously recorded in municipality documents such as maps, maintenance records and daily field logs. In addition to cities and utilities' documents mentioned above, staff interviews, survey questionnaires, and informal meetings are always good sources for information. Often compilation of this information is in paper format which limits the readily accessibility. Today, the most effective format for storage wastewater pipe infrastructure data would be an electronic overall base map such as Geographic Information System (GIS). Wastewater pipeline data can be collected by direct or indirect methods, for example, direct methods such as various inspection methods, and indirect methods such as hydraulic modeling. For example, one of the most widely used inspection techniques for 
collecting internal wastewater pipeline information is called Closed-Circuit Television (CCTV). From CCTV images the defects in the pipe such as cracks, fractures and holes are captured. Root intrusions through the cracked or broken pipe, Infiltration/ Inflow (I/I) and exfiltration can be detected via this technique as well. Other effective inspection techniques include smoke test, dye test, and manhole inspection.

Data collection of pipe parameters can be time consuming and require expensive testing; therefore, selection of parameter collection techniques should be considered. To aid in the collection methods and protocols, a comprehensive list of parameter sources was compiled to give users the various collection techniques. Tables 4.2 and 4.3 illustrate each wastewater pipe parameter and list of possible collection sources. 
Table 4.2 A to L Parameter Sources

\begin{tabular}{|c|c|c|c|c|c|c|c|c|c|c|}
\hline Parameter & 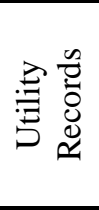 & 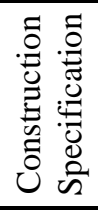 & 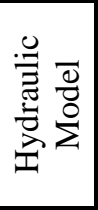 & 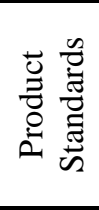 & 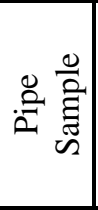 & 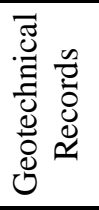 & 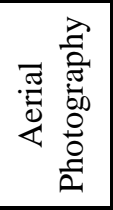 & 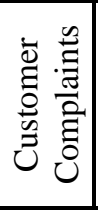 & 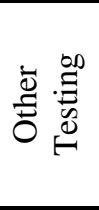 & 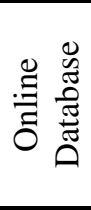 \\
\hline Age & $\mathrm{X}$ & $\mathrm{X}$ & & & & & & & & \\
\hline Backup Flooding & $\mathrm{X}$ & & $X$ & & & & & $\mathrm{X}$ & & \\
\hline Bedding Condition & $\mathrm{X}$ & $\mathrm{X}$ & & & & & & & $\mathrm{X}$ & \\
\hline Blockage & $\mathrm{X}$ & & $\mathrm{X}$ & & & & & $\mathrm{X}$ & $\mathrm{X}$ & \\
\hline Cathodic Protection & $\mathrm{X}$ & $\mathrm{X}$ & & & & & & & & \\
\hline Closeness to Trees & $\mathrm{X}$ & & & & & & $\mathrm{X}$ & & & \\
\hline Coating & $\mathrm{X}$ & $X$ & & $\mathrm{X}$ & $\mathrm{X}$ & & & & & \\
\hline Condition & $\mathrm{X}$ & & & & & & & & & \\
\hline Connection Density & $\mathrm{X}$ & $\mathrm{X}$ & & & & & & & & \\
\hline Cover Depth & $\mathrm{X}$ & $X$ & & & & & & & $\mathrm{X}$ & \\
\hline Design Life & $\mathrm{X}$ & & & $\mathrm{X}$ & & & & & & \\
\hline Diameter & $\mathrm{X}$ & $\mathrm{X}$ & & $\mathrm{X}$ & $\mathrm{X}$ & & & & & \\
\hline Dissimilar Materials/Metals & $\mathrm{X}$ & $\mathrm{X}$ & & & & & & & & \\
\hline Disturbances & $\mathrm{X}$ & & & & & & & & & \\
\hline Exfiltration & $\mathrm{X}$ & & $\mathrm{X}$ & & & & & & $\mathrm{X}$ & \\
\hline Extreme Temperatures & $\mathrm{X}$ & & & & & & & & $\mathrm{X}$ & $\mathrm{X}$ \\
\hline Failure Utilities & $\mathrm{X}$ & & & & & & & & & \\
\hline FOG & $\mathrm{X}$ & & & & & & & $\mathrm{X}$ & & \\
\hline Flooding & $\mathrm{X}$ & & & & & & $\mathrm{X}$ & & & $\mathrm{X}$ \\
\hline Flow Velocity & $\mathrm{X}$ & & $X$ & & & & & & $\mathrm{X}$ & \\
\hline Frost Penetration & $\mathrm{X}$ & & & & & & & & $X$ & $\mathrm{X}$ \\
\hline Function & $\mathrm{X}$ & $\mathrm{X}$ & & & & & & & & \\
\hline Groundwater Table & $\mathrm{X}$ & & & & & $\bar{X}$ & & & $\mathrm{X}$ & $\mathrm{X}$ \\
\hline $\mathrm{H}_{2} \mathrm{~S}$ & $\mathrm{X}$ & & & & & & & & $X$ & \\
\hline $\mathrm{I} \& \mathrm{I}$ & $\mathrm{X}$ & & $\mathrm{X}$ & & & & & & $\mathrm{X}$ & \\
\hline Installation & $\mathrm{X}$ & & & & & & & & & \\
\hline Joint Type & $\mathrm{X}$ & $\mathrm{X}$ & & $\mathrm{X}$ & & & & & & \\
\hline Lateral & $\mathrm{X}$ & $\mathrm{X}$ & & & & & & & & \\
\hline Length & $\mathrm{X}$ & $\mathrm{X}$ & & & & & & & & \\
\hline Lining & $\mathrm{X}$ & $\mathrm{X}$ & & $\mathrm{X}$ & $\mathrm{X}$ & & & & & \\
\hline Load & $X$ & & & & & & & & $\mathrm{X}$ & \\
\hline
\end{tabular}


Table 4.3 M to Z Parameter Sources

\begin{tabular}{|c|c|c|c|c|c|c|c|c|c|c|}
\hline Parameter & 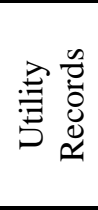 & 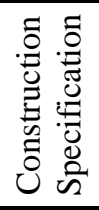 & 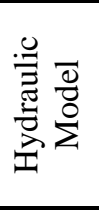 & 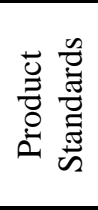 & 总 & 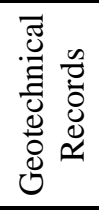 & 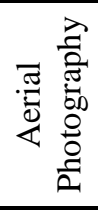 & 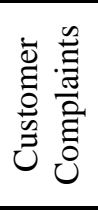 & 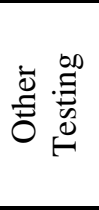 & 咅芯 \\
\hline Manhole & $\mathrm{X}$ & $\mathrm{X}$ & & & & & & & & \\
\hline Manufacture & $\mathrm{X}$ & $\mathrm{X}$ & & & & & & & & \\
\hline Material Type & $X$ & $\mathrm{X}$ & & & $\mathrm{X}$ & & & & & \\
\hline Moisture Content & $\mathrm{X}$ & & & & & $\mathrm{X}$ & & & $\mathrm{X}$ & \\
\hline Odors & $\mathrm{X}$ & & & & & & & $\mathrm{X}$ & $\mathrm{X}$ & \\
\hline Operational Pressure & $X$ & & $\mathrm{X}$ & & & & & & $X$ & \\
\hline Overflow & $\mathrm{X}$ & & $\mathrm{X}$ & & & & & $\mathrm{X}$ & $\mathrm{X}$ & \\
\hline Precipitation & $\mathrm{X}$ & & & & & & & & & $\mathrm{X}$ \\
\hline Seismic Activity & $\mathrm{X}$ & & & & & & & & & $\mathrm{X}$ \\
\hline Slope & $X$ & $\mathrm{X}$ & & & & & $\mathrm{X}$ & & & $\mathrm{X}$ \\
\hline Soil Corrosivity & $\mathrm{X}$ & & & & & $\mathrm{X}$ & & & $\mathrm{X}$ & \\
\hline Soil pH & $\mathrm{X}$ & & & & & $\mathrm{X}$ & & & $\mathrm{X}$ & \\
\hline Soil Redox Potential & $X$ & & & & & $\mathrm{X}$ & & & $X$ & \\
\hline Soil Resistivity & $\mathrm{X}$ & & & & & $\mathrm{X}$ & & & $\mathrm{X}$ & \\
\hline Soil Sulfides & $\mathrm{X}$ & & & & & $\mathrm{X}$ & & & $\mathrm{X}$ & \\
\hline Soil Type & $\mathrm{X}$ & & & & & $\mathrm{X}$ & & & $\mathrm{X}$ & $\mathrm{X}$ \\
\hline Stray Currents & $X$ & $\mathrm{X}$ & & & & $\mathrm{X}$ & & & $X$ & \\
\hline Surcharging & $\mathrm{X}$ & & $\mathrm{X}$ & & & & & & & \\
\hline Tidal Influences & $\mathrm{X}$ & & & & & $\mathrm{X}$ & $\mathrm{X}$ & & $\mathrm{X}$ & $\mathrm{X}$ \\
\hline Thrust Restraint & $X$ & $\mathrm{X}$ & & $\mathrm{X}$ & $\mathrm{X}$ & & & & & \\
\hline Trench Backfill & $\mathrm{X}$ & $\mathrm{X}$ & & & & & & & $\mathrm{X}$ & \\
\hline Trench Depth & $\mathrm{X}$ & $\mathrm{X}$ & & & & & & & $\mathrm{X}$ & \\
\hline Trench Width & $X$ & $\mathrm{X}$ & & & & & & & $\mathrm{X}$ & \\
\hline Type of Cleaning & $\mathrm{X}$ & & & & & & & & & \\
\hline Vintage & $\mathrm{X}$ & $\mathrm{X}$ & & $\mathrm{X}$ & & & & & & \\
\hline Wall Thickness & $\mathrm{X}$ & & & $\mathrm{X}$ & $\mathrm{X}$ & & & & & \\
\hline Wastewater Quality & $\mathrm{X}$ & & & & & & & & $\mathrm{X}$ & \\
\hline Wet/Dry Cycles & $X$ & & & & & & & & & $X$ \\
\hline
\end{tabular}




\subsection{Parameters}

All parameters for a wastewater pipe system, including physical/structural, operational/functional, environmental, and other parameters, must be included in developing a performance index for wastewater pipes, and the entire system must be considered, not just the pipe. Some parameters that could have affected wastewater pipes may be missing, but we have summarized most of the essential parameters based on literature review, interviews with utility and pipe association representatives, and questionnaires. Parameters that were used in the weighted factor model and the fuzzy inference model are shown in Table 4.4.

Table 4.4 Input Parameters

\begin{tabular}{|r|l|l|}
\hline No. & Parameters & Unit \\
\hline 1 & Bedding Condition & Level \\
\hline 2 & D/d (flow depth over diameter) & $\%$ \\
\hline 3 & Density of Connections & level \\
\hline 4 & Flooding (in an area prone to flooding) & Yes/no \\
\hline 5 & Flow Velocity & f/s \\
\hline 6 & Frost Penetration & Yes/no \\
\hline 7 & Ground Cover & Type \\
\hline 8 & Groundwater Table & level \\
\hline 9 & H2S & Ppm \\
\hline 10 & Location (Traffic) & level \\
\hline 11 & Maintenance Frequency & Level \\
\hline 12 & Pipe Age & Year \\
\hline 13 & Pipe Condition & Level \\
\hline 14 & Pipe Depth & $\mathrm{ft}$ \\
\hline 15 & Pipe Diameter & inch \\
\hline 16 & Pipe Length & $\mathrm{ft}$ \\
\hline 17 & Pipe Slope & $\%$ \\
\hline 18 & Pipe Surcharging & Level \\
\hline 19 & Pipe Wall Thickness & $\%$ Loss \\
\hline 20 & Proximity to trees & $\mathrm{ft}$ \\
\hline 21 & Redox Potential & $\mathrm{mV}$ \\
\hline 22 & Soil Chloride & ppm \\
\hline 23 & Soil Disturbance & Yes/No \\
\hline 24 & Soil pH & $\mathrm{pH}$ \\
\hline 25 & Soil Resistivity & Ohm-cm (Level) \\
\hline 26 & Soil Sulfate & ppm \\
\hline & & \\
\hline
\end{tabular}




\begin{tabular}{|r|l|l|}
\hline No. & Parameters & Unit \\
\hline 27 & Soil Type & Type \\
\hline 28 & Stray Currents & Yes/no \\
\hline 29 & Tidal Influences & Yes/no \\
\hline 30 & Type of Cleaning & Level \\
\hline 31 & Wastewater $\mathrm{pH}$ & $\mathrm{pH}$ \\
\hline 32 & Wastewater Sulfate & $\mathrm{mg} / \mathrm{l}$ \\
\hline
\end{tabular}

A brief description of each parameter and its effect are described as follows,

\section{B}

Bedding Condition-A pipe is not made to act as a load-bearing beam and must be supported through adequate bedding. The bedding should be a uniform support made up of clean backfill that is properly tamped to reduced settling and shifting. Uneven support due to lack of proper bedding condition can lead to beam stress on the pipe (Smith el al., 2000). The unit of this parameter is condition level. This parameter is important for all pipe materials.

\section{D}

D/d (flow depth over diameter)-This parameter is the ratio of flow depth over pipe diameter. When the sewer flow is much greater than the capacity of the pipe, the overflow can occur. Sewer overflow happens when the pipe is blocked and results in backup flooding into building basement or street through inlets and manholes. Usually, backup flooding is catastrophic and may contaminate the environment. However, if the flow is constantly low, it may lead to corrosion problem. The unit of this parameter is $0-1$. This parameter is important for all pipe materials.

Density of Connection-The required wastewater capacity is dependent of the number of service connections. The pipe size, and flow rate of the collection mains must be adequate to transport wastewater to the treatment plants. The unit of this parameter is number of lateral connection/100ft. This parameter is important for all pipe materials. (See Diameter and Flow Velocity)

$\mathbf{F}$

Flooding-Flooding can impact the pipe and soil equilibrium causing the pipe to collapse or float out of alignment. Aggressive waters and/or constant water in contact with the pipe can increase 
the external corrosion rate (Whidden, 2009). The unit of this parameter is in occurrence level. This parameter is important for all pipe materials.

Flow Velocity-The flow of the fluid through the wastewater pipe. Flow velocity can determine the pipe capacity and required pipe diameter during pipe design. Velocities should not be less than 2 $\mathrm{ft} / \mathrm{s}$ which causing sediment to build up. However, excessive velocities are not recommended, because it leads to mechanical surface wears and exposing of aggregates in concrete pipes. The unit of this parameter is $\mathrm{ft} / \mathrm{s}$. This parameter is important for all pipe materials.

Frost Penetration-Frost penetration is the depth to which frost can penetrate the soil. The greater depth of frost penetration increases the earth loading on the pipe. The increase in dead load results in a compressive stress or crushing force which acts on the pipe, leading to potential longitudinal cracks (Smith et al., 2000). A flexible pipe tend to have lesser load increased due to frost penetration than on a rigid pipe (Moser, 2001). The unit of this parameter is yes/no. This parameter is important for all pipe materials.

\section{G}

Ground Cover-Different ground covers has different impacts to the pipe. Some soil cover may prevent erosion or run-off better than others. Pipe located under concrete or asphalt may subject to different loads. The unit of this parameter is Type (ex., concrete, bare soil, grass). This parameter is important for all pipe materials.

Groundwater Table-The location of the groundwater table can affect the pipe-soil relationship. If the pipe is located at or below the water table, floatation may occur if proper measures are not considered such as a greater soil cover or weighting system. Minimum soil cover for flotation is $H=D$, where; $H=$ rutted height of soil cover and $D=$ mean diameter of pipe. Constant contact with groundwater may lead to external corrosion in metal pipes (Moser, 2001). The unit of this parameter is $f t$. This parameter is important for metal pipes and reinforced concrete pipes when crack is present. 


\section{$\mathbf{H}$}

Hydrogen Sulfide Gas $\left(\mathrm{H}_{2} \mathrm{~S}\right)$-High level of $\mathrm{H}_{2} \mathrm{~S}$ concentration can severely cause pipe corrosion and eventually lead to structural failure. Sulfuric acid that corrodes the pipe near the crown is formed by hydrogen sulfide gas in sewage. $\mathrm{H}_{2} \mathrm{~S}$ usually occurs in shallow slope pipes. The unit of this parameter is level, or ppm. This parameter is important for metal and concrete pipes.

\section{$\mathbf{L}$}

Location (traffic)-Live loading from traffic can cause compressive forces on the pipe wall. This downward pressure is a factor of the pipe depth, soil type, type of pavement (rigid or flexible) and the type of vehicles. (See Depth and Soil Type) Excessive crushing forces can lead to longitudinal cracks on the pipe wall. Bending stresses are also present within the pipe if the pipeline is not evenly supported. (See Bedding Condition) Excessive bending stress can lead to circumferential cracking (Smith et al., 2000). The unit of this parameter is average daily traffic (ADT), or level. This parameter is important for all pipe materials.

\section{M}

Maintenance Frequency-Frequency of maintenance significantly affect the operational and functional condition of the pipe. Rarely or no maintenance may lead to root or blockage problems. (See Blockage) The unit of this parameter is level (ex., rarely, regularly). This parameter is important for all pipe materials.

\section{$\mathbf{P}$}

Pipe Age-The length of time since the asset was installed. Age may or may not be a strong indicator of pipeline deterioration and can be a function of the material type and other factors. Typically, it is assumed that older pipes have sustained longer stresses and may subject to deterioration with age. The unit of this parameter is year. This parameter is important for all pipe materials.

Pipe Condition-Pipe condition can be determined by different types of inspection. Typical inspection methods used to examine the pipe are CCTV inspection, smoke test, and dye test. The unit of this parameter is in condition scale 1-5. This parameter is important for all pipe materials. 
Pipe Depth-The pipe depth can play a factor in the amount of stress applied to the pipe as a result of live and dead load loading. As the soil cover increases, the load pressure decreases (Moser, 2001). The amount of cover can also influence the type of failure mode and mechanism. For example, if the pipe is buried at sufficient depth it is more likely to develop localize buckling than beam buckling (Smith et al., 2000). A shallow pipe depth may also be a factor for third party damages. The unit of this parameter is $f t$. This parameter is important for all pipe materials.

Pipe Diameter-The diameter of a pipeline is typically classified by the nominal diameter or outside diameter rather than the inside diameter. Small diameter pipelines are more susceptible to beam failure than larger pipe diameters. In relation to pipe capacity, smaller diameters are easy to be blocked by object, root intrusion, or built- up sediment. The unit of this parameter is Inch. This parameter is important for all pipe materials.

Pipe Length-There are two lengths regarding wastewater pipeline, a section length between joints and, a node length between manholes. An increase in pipe section length can lead to increased stresses as a result of differential ground movement transverse to the pipe axis. A pipeline that is not properly supported can result in beam stresses. Excessive beam stress can lead to circumferential cracking (Smith et al., 2000). A pipe with node length greater than 500ft is considered difficult to maintain due to the maintaining equipment operating length. The unit of this parameter is $f t$. This parameter is important for all pipe materials.

Pipe Slope-The pipe slope affects the velocity of a gravity flow sewer and may result in blockage, sediment, and corrosion. Pipe slope may be derived from upstream invert elevation, downstream invert elevation, and pipe length. The unit of this parameter is in gradient. This parameter is important for all pipe materials.

Pipe Surcharging-Surcharging in a gravity sewer in dry \& wet weather should be monitored. Surcharge describes as a condition that the sewer flows full and under pressure. Surcharge occur as a result of under design capacity or changing of system condition such as deposit or blockage. Surcharging usually occurs during storm due to high groundwater table. Surcharging is usually 
measured in hydraulic head level (ft). The units of this parameter are yes/no, andft. This parameter is important for all pipe materials.

Pipe Wall Thickness-The pipe wall thickness often governs the operational pressure of the pipe and is variable on pipe diameter and material type. (See Diameter) The magnitude of potential pipe stresses in relation to loading and depth of pipe can also be a function of the wall thickness. (See Location (traffic) and Depth) Thickness is also a variable in analyzing the potential of corrosion and can dictate the amount of time corrosion pitting can be detrimental towards the pipe lifespan (Stathis, 1998). The unit of this parameter is Inch. This parameter is important for all pipe materials.

Proximity to Trees-A pipe in proximity to the trees may be subject to root intrusion, if there is a crack or a hole presented in the pipe. Tree root may also seep through an open or separate joint. The unit of this parameter is $f t$. This parameter is important for all pipe materials.

\section{$\mathbf{R}$}

Redox Potential-Redox potential (also known as the reduction potential) is the measure of the tendency of the soil to attain electrons. Substances that accept electrons are capable of reducing, having a negative redox potential which indicates an anaerobic condition. Soils that are anaerobic are regarded as potentially corrosive predominately in ferrous material pipelines (AWWA, 2005). The unit of this parameter is $m V$. This parameter is important for metal and concrete pipes.

\section{$\mathbf{S}$}

Soil Chloride-Soil chloride is highly soluble and usually found dissolved in the soil water. Concentration level can be measured from extracted water of the soil. Highly chloride concentration level in high $\mathrm{pH}$ environments can lead to serious corrosion in the pipe. The unit of this parameter is ppm. (See Soil Resistivity) This parameter is important for metal and concrete pipes.

Soil Disturbance-Third party disturbances to the pipeline can lead to direct or indirect damage. For example construction disturbances due to excavation can lead to direct damage which results 
in the equipment physically breaking the pipe or indirect damage due to soil movement close to the pipe. Disturbances in the pipe bedding or alignment can lead to beam failure if the pipe is not adequately supported and/or the pipe depth is not sufficient (Smith et al., 2000). The unit of this parameter is yes/no. This parameter is important for all pipe materials.

Soil $\mathrm{pH}$-Soil $\mathrm{pH}$ is the measure of the soil acidity or alkalinity. A low $\mathrm{pH}$ represents an acidic soil promoting corrosion and is also a soil that serves well as an electrolyte. High alkaline conditions can also lead to corrosion of a ferrous material pipeline since they are a soil that is high in dissolved salts which yield a low soil resistivity (AWWA, 2005). The unit of this parameter is $p H$ level. (See Soil Resistivity) This parameter is important for metal and concrete pipes.

Soil Resistivity-Soil resistivity is a measure of the soil to serve as an electrolyte and is often measured in the presence of ferrous material pipelines. The lower the resistivity value the more likely the soil will serve as an electrolyte which relates to an increase in soil corrosion activity. The soil temperature does have an impact on the soil resistivity; as the temperature decreases, the soil resistivity increases. Soil resistivity is also a function of the soil moisture content; the higher the moisture content, the lower the soil resistivity (Kliener, 2010). The unit of this parameter is Ohm-cm, level. This parameter is important for metal and concrete pipes.

Soil Sulfate-Soils containing sulfate indicate there is a problem caused by sulfate-reducing bacteria. Soils with positive sulfate content are more prone to pipeline corrosion (AWWA, 2005). When a pipe is contnuously presented in high sulfate concentrations, calcium in concrete reacts with sulfate and can make the concrete pipe weak. High concentrations of sulfate in groundwater can be found in the particular area such as the area under influence of geothermal or volcanic geology. If the high sulfate groundwater enters a pipe, it can increase the rate of microbiological influenced corrosion same as intrustrial discharge (Bizier, 2007). The unit of this parameter is percent. This parameter is important for metal and concrete pipes.

Soil Type-The soil dead load and change in soil volume can be attributed to differing types of soil. The unit weight of the soil and depth of the pipe conclude the total dead load resulting from the soil. (See Depth) This external load is usually accountable for ring deflection of the pipe. Soil 
types that are compressible can result in a greater pipe deflection due to additional loading. Certain soil types are also more prone to expansion and contraction of soil due to the wetting and drying cycles. In particular, the expansion of the soil can cause the external soil load to increase which can result in axial and/or beam loads on the pipeline (Moser, 2001). The unit of this parameter is type (i.e., clay, sand). This parameter is important for all pipe materials.

Stray Currents-Stray currents are caused by a local direct current (DC) flowing through the earth. These stray currents can be present if the pipe is nearby a transportation system such as a railway or if other utilities are close to the pipe. Often these stray electrical currents can cause electrolytic corrosion in metal pipelines if they are not properly protected. Cathodic protection is the most common protection method of metal pipelines as a result of stray currents. The unit of this parameter is yes/no.

\section{$\mathbf{T}$}

Tidal Influences-Tidal influences within coastal areas can influence the soil groundwater table. As the wave progresses inland as a result of high tide, the groundwater table can fluctuate (Smith, 1994). The unit of this parameter is yes/no. (See Groundwater Table) This parameter is important for all pipe materials.

Type of Cleaning-Some type of cleaning may damage the pipe. Typical cleaning methods used for wastewater pipe are water jetting, bucketing, and chemical. The unit of this parameter is type (i.e., jetting). This parameter is important for all pipe materials.

\section{W}

Wastewater Quality-Wastewater quality is a function of several properties such as wastewater $\mathrm{pH}$, $\mathrm{BOD}, \mathrm{COD}$, temperature, and chemical. The wastewater quality is thought to have an effect on the corrosion. This parameter is important for all pipe materials. 


\subsection{Weighted Factor Performance Index Evaluation (W-PIE)}

The model evaluates each parameter and combines them mathematically through a weighted summation that reflects importance of various factors. The W-PIE model utilizes weighted factor, which is a transparent method to show the calculation, and requires less effort if any modification is needed. The model evaluates each parameter and gives the score in scale from zero to five based on the parameter ranges presented in Table 4.5. The parameter ranges were established based on prior research knowledge and utility feedbacks and inputs.

\section{Table 4.5 Parameter Ranges}

\begin{tabular}{|c|c|c|c|}
\hline No. & Parameter & Unit & Range \\
\hline \multirow[t]{6}{*}{1} & Pipe Age & Year & If age $=0-15 ; 0$ \\
\hline & & & If age $=15-30 ; 1$ \\
\hline & & & If age $=30-45 ; 2$ \\
\hline & & & If age $=45-60 ; 3$ \\
\hline & & & If age $=60-75 ; 4$ \\
\hline & & & If age $=>75 ; 5$ \\
\hline \multirow[t]{6}{*}{2} & Pipe Depth & $\mathrm{ft}$ & If Depth $=>18 ; 0$ \\
\hline & & & If Depth = 12-18;1 \\
\hline & & & If Depth $=9-12 ; 2$ \\
\hline & & & If Depth $=6.5-12 ; 3$ \\
\hline & & & If Depth $=4-6.5 ; 4$ \\
\hline & & & If Depth $<4 ; 5$ \\
\hline \multirow[t]{6}{*}{3} & Pipe Diameter & inch & $6-12 ; 5$ \\
\hline & & & $12-18 ; 4$ \\
\hline & & & $18-24 ; 3$ \\
\hline & & & $24-36 ; 2$ \\
\hline & & & $36-60 ; 1$ \\
\hline & & & $>60 ; 0$ \\
\hline \multirow[t]{3}{*}{4} & Pipe Length & $\mathrm{ft}$ & $0-100 ; 0$ \\
\hline & & & $100-200 ; 1$ \\
\hline & & & $200-300 ; 2$ \\
\hline
\end{tabular}




\begin{tabular}{|c|c|c|c|}
\hline No. & Parameter & Unit & Range \\
\hline & & & $300-400 ; 3$ \\
\hline & & & $400-500 ; 4$ \\
\hline & & & $>500 ; 5$ \\
\hline \multirow[t]{4}{*}{5} & Pipe Slope & $\%$ & $>2 ; 0$ \\
\hline & & & $1-2 ; 3$ \\
\hline & & & $0.5-1 ; 4$ \\
\hline & & & $<0.5$ or $>5 ; 5$ \\
\hline \multirow[t]{4}{*}{6} & Pipe Wall Thickness & $\%$ Loss & $10 \% ; 1$ \\
\hline & & & $10 \%-20 \% ; 2$ \\
\hline & & & $20 \%-30 \% ; 3$ \\
\hline & & & $>30 \% 5$ \\
\hline \multirow[t]{6}{*}{7} & D/d (flow depth over & $0-1$ & $>=1$ full $; 5$ \\
\hline & diameter) & & $0.75-1 ; 4$ \\
\hline & & & $0.5-0.75 ; 3$ \\
\hline & & & $0.25-0.5 ; 2$ \\
\hline & & & $0.1-0.25 ; 1$ \\
\hline & & & $<0.1 ; 0$ \\
\hline \multirow[t]{6}{*}{8} & Flow Velocity & $\mathrm{f} / \mathrm{s}$ & $<2 ; 5$ \\
\hline & & & $2.0-2.5 ; 4$ \\
\hline & & & $2.5-3 ; 3$ \\
\hline & & & $3-4 ; 2$ \\
\hline & & & $4-5 ; 1$ \\
\hline & & & $>5 ; 0$ \\
\hline \multirow[t]{6}{*}{9} & $\mathrm{H} 2 \mathrm{~S}$ & $\mathrm{Ppm}$ & $0-25 ; 0$ \\
\hline & & & $25-50 ; 1$ \\
\hline & & & $50-100 ; 2$ \\
\hline & & & $100-250 ; 3$ \\
\hline & & & $250-500 ; 4$ \\
\hline & & & $>500 ; 5$ \\
\hline
\end{tabular}




\begin{tabular}{|c|c|c|c|}
\hline No. & Parameter & Unit & Range \\
\hline 10 & Pipe Surcharging & level & $\begin{array}{l}\text { Frequent (weekly, monthly) \& high magnitude } ; 5 \\
\text { Frequent \& low magnitude ;4 } \\
\text { Occasional (1- } 12 \text { times per year) \& high magnitude; ;3 } \\
\text { Occasional - low magnitude ;2 } \\
\text { no; } 0\end{array}$ \\
\hline 11 & Wastewater $\mathrm{pH}$ & $\mathrm{pH}$ & $\begin{array}{l}<5.5 ; 5 \\
5.5-6 ; 3 \\
6-9 ; 0 \\
9-9.5 ; 3 \\
>9 ; 5\end{array}$ \\
\hline 12 & Wastewater Sulfate & $\mathrm{mg} / \mathrm{l}$ & $\begin{array}{l}>200 ; 5 \\
<200 ; 0\end{array}$ \\
\hline 13 & Bedding Condition & Level & $\begin{array}{l}\text { Excellent (class A), } 0 \\
\text { Good (class B), } 1 \\
\text { Fair (class C), } 3 \\
\text { Poor(class D), } 5\end{array}$ \\
\hline 14 & $\begin{array}{l}\text { Density of Connection } \\
\text { (number of lateral } \\
\text { connection) }\end{array}$ & level & $\begin{array}{l}\text { Very Dense ( }>5 \text { per } 100 \mathrm{ft}), 5 \\
\text { Dense ( } 4-5 \text { per } 100 \mathrm{ft}) ; 4 \\
\text { Medium Dense }(3-5 \text { per } 100 \mathrm{ft}), 3 \\
\text { Medium }(2-3 \text { per } 100 \mathrm{ft}) ; 2 \\
\text { Light }(1-2 \text { per } 100 \mathrm{ft}) ; 1 \\
\text { Very Light }(<1 \text { per } 100 \mathrm{ft}) ; 0\end{array}$ \\
\hline 15 & $\begin{array}{l}\text { Flooding } \\
\text { (in an area prone to } \\
\text { flooding) }\end{array}$ & Yes/no & $\begin{array}{l}\text { Yes;5 } \\
\text { No; } 0\end{array}$ \\
\hline 16 & Frost Penetration & Yes/no & $\begin{array}{l}\text { Yes;5 } \\
\text { No; } 0\end{array}$ \\
\hline
\end{tabular}




\begin{tabular}{|c|c|c|c|}
\hline No. & Parameter & Unit & Range \\
\hline 17 & Ground Cover & Type & $\begin{array}{l}\text { Asphalt, concrete; } 0 \\
\text { unpaved road } 1 \\
\text { gravel, grass; } 3 \\
\text { dirt, loose particle material; } 5\end{array}$ \\
\hline 18 & Groundwater Table & level & $\begin{array}{l}\text { Below pipe; } 0 \\
\text { close to pipe }<2 \mathrm{ft} ; 3 \\
\text { Slightly above } 2-5 \mathrm{ft} ; 4 \\
\text { above pipe }>4 \mathrm{ft} ; 5\end{array}$ \\
\hline 19 & Location (Traffic) & level & $\begin{array}{l}\text { light }>50 \mathrm{ft} \text { from road or railway } ; 0 \\
\text { medium } 50 \mathrm{ft} \text { from road or railway } ; 3 \\
\text { heavy } 20 \mathrm{ft} \text { from major road or railway;5 }\end{array}$ \\
\hline 20 & Proximity to trees & $\mathrm{ft}$ & $\begin{array}{l}>20 ; 0 \\
15-20 ; 1 \\
10-15 ; 3 \\
0-5 ; 5\end{array}$ \\
\hline 21 & Redox Potential & $\mathrm{mV}$ & $\begin{array}{l}-40-0 ; 0 \\
-40--60 ; 2 \\
-60--80 ; 4 \\
<-100 ; 5\end{array}$ \\
\hline 22 & Soil Chloride & ppm & $\begin{array}{l}>350 ; 5 \\
<350 ; 0\end{array}$ \\
\hline 23 & Soil Disturbance & Yes/No & $\begin{array}{l}\text { Yes;5 } \\
\text { No; } 0\end{array}$ \\
\hline 24 & Soil pH & $\mathrm{pH}$ & $\begin{array}{l}<5.5 ; 5 \\
5.5-6 ; 3 \\
6-9 ; 0 \\
9-9.5 ; 3\end{array}$ \\
\hline
\end{tabular}




\begin{tabular}{|c|c|c|c|}
\hline No. & Parameter & Unit & Range \\
\hline & & & $>9 ; 5$ \\
\hline \multirow[t]{5}{*}{25} & Soil Resistivity & $\begin{array}{l}\text { Ohm- } \\
\mathrm{cm}\end{array}$ & 0-1000 extremely corrosive (corrosion activity);5 \\
\hline & & (Level) & $1000-3000$ very corrosive $; 4$ \\
\hline & & & $3000-5000$ corrosive $; 3$ \\
\hline & & & 5000-10000 moderately corrosive; 1 \\
\hline & & & $>=10000$ mildly corrosive $; 0$ \\
\hline \multirow[t]{4}{*}{26} & Soil Sulfate & $\mathrm{ppm}$ & $>5000 ; 5$ \\
\hline & & & $3000-5000 ; 4$ \\
\hline & & & $1000-3000 ; 3$ \\
\hline & & & $<1000 ; 0$ \\
\hline \multirow[t]{5}{*}{27} & Soil Type & Type & gravel, 0 \\
\hline & & & coarse sand, 1 \\
\hline & & & fine sand and silt, 3 \\
\hline & & & low plasticity clay $(\mathrm{PI}<15), 4$ \\
\hline & & & high plasticity clay; 5 \\
\hline \multirow[t]{2}{*}{28} & Stray Currents & Yes/no & Yes;5 \\
\hline & & & No; 0 \\
\hline \multirow[t]{2}{*}{29} & Tidal Influences & Yes/no & Yes;5 \\
\hline & & & No; 0 \\
\hline \multirow[t]{4}{*}{30} & Maintenance Frequency & Level & Very Often, cleaning and Inspecting every $1-3$ years; 0 \\
\hline & & & Regularly, $3-5$ years; 2 \\
\hline & & & Rarely, $>5$ years; 4 \\
\hline & & & Never;5 \\
\hline \multirow[t]{3}{*}{31} & Type of Cleaning & Type & Best ex, Jetting; 0 \\
\hline & & & Moderate ex, Rodding; 3 \\
\hline & & & Worst ex, Bucketing; 5 \\
\hline
\end{tabular}


The weights assigned to each parameter were created from questionnaires giving to participating utilities. The questionnaire was made up of rating the significance of each parameter on a scale one to five. The value of one represents the most significance, whereas the value of five represents the least significance. The feedbacks were reviewed and the weight of each parameter was assigned. The weights were sent to experts for review and validation. A list of a weight given to each parameter is summarized in Tables 4.6 and 4.7 by module. We would like to note that weights and ranges can be modified based on additional knowledge and/or experience.

Table 4.6 Parameter Weight by Module (Structural Index)

\begin{tabular}{|c|c|c|c|c|}
\hline Parameters & $\begin{array}{l}\text { Concrete } \\
(\%)\end{array}$ & $\begin{array}{l}\text { Clay } \\
(\%)\end{array}$ & $\begin{array}{l}\text { Metal } \\
(\%)\end{array}$ & $\begin{array}{l}\text { Plastic } \\
(\%)\end{array}$ \\
\hline \multicolumn{5}{|c|}{ 1. External Corrosion Module } \\
\hline Groundwater Table & 10 & 0 & 10 & 0 \\
\hline Pipe Age & 25 & 0 & 20 & 0 \\
\hline Pipe Depth & 5 & 0 & 5 & 0 \\
\hline Soil Resistivity & 15 & 0 & 20 & 0 \\
\hline Pipe Wall Thickness & 15 & 0 & 5 & 0 \\
\hline Soil pH & 5 & 0 & 5 & 0 \\
\hline Soil Sulfate & 10 & 0 & 5 & 0 \\
\hline Soil Chloride & 5 & 0 & 10 & 0 \\
\hline Redox Potential & 10 & 0 & 10 & 0 \\
\hline Stray Currents & 0 & 0 & 10 & 0 \\
\hline \multicolumn{5}{|c|}{ 2. Internal Corrosion Module } \\
\hline Pipe Slope & 10 & 0 & 10 & 0 \\
\hline Pipe Age & 20 & 0 & 20 & 0 \\
\hline Flow Velocity & 10 & 0 & 10 & 0 \\
\hline Flow depth/Diameter (d/D) & 10 & 0 & 10 & 0 \\
\hline Wastewater $\mathrm{pH}$ & 5 & 0 & 5 & 0 \\
\hline Wastewater Sulfate & 5 & 0 & 5 & 0 \\
\hline $\mathrm{H} 2 \mathrm{~S}$ & 30 & 0 & 30 & 0 \\
\hline Maintenance Frequency & 10 & 0 & 10 & 0 \\
\hline
\end{tabular}




\begin{tabular}{|c|c|c|c|c|}
\hline Parameters & $\begin{array}{l}\text { Concrete } \\
(\%)\end{array}$ & $\begin{array}{l}\text { Clay } \\
(\%)\end{array}$ & $\begin{array}{l}\text { Metal } \\
(\%)\end{array}$ & $\begin{array}{l}\text { Plastic } \\
(\%)\end{array}$ \\
\hline \multicolumn{5}{|c|}{ 3. Surface Wear Module } \\
\hline Pipe Age & 30 & 30 & 30 & 30 \\
\hline Flow Velocity & 20 & 20 & 20 & 20 \\
\hline Pipe Slope & 20 & 20 & 20 & 20 \\
\hline Type of Cleaning & 30 & 30 & 30 & 30 \\
\hline \multicolumn{5}{|l|}{ 4. Stress Module } \\
\hline Pipe Age & 25 & 10 & 25 & 20 \\
\hline Pipe Depth & 10 & 10 & 5 & 5 \\
\hline \multicolumn{5}{|l|}{$\underline{\text { Support }}$} \\
\hline Bedding Condition & 10 & 15 & 10 & 15 \\
\hline Soil Disturbance & 10 & 10 & 10 & 10 \\
\hline Flooding & 10 & 10 & 10 & 10 \\
\hline Tidal Influences & 5 & 5 & 5 & 5 \\
\hline \multicolumn{5}{|l|}{$\underline{\text { Soil Load }}$} \\
\hline Frost Penetration & 5 & 5 & 5 & 5 \\
\hline Groundwater Table & 5 & 5 & 5 & 5 \\
\hline \multicolumn{5}{|l|}{$\underline{\text { Traffic Load }}$} \\
\hline Location (Traffic) & 15 & 20 & 15 & 15 \\
\hline Ground Cover & 5 & 10 & 10 & 10 \\
\hline
\end{tabular}


Table 4.7 Parameter Weight by Module (Functional Index)

\begin{tabular}{|c|c|c|c|c|}
\hline Parameters & $\begin{array}{l}\text { Concrete } \\
(\%)\end{array}$ & $\begin{array}{l}\text { Clay } \\
(\%)\end{array}$ & $\begin{array}{l}\text { Metal } \\
(\%)\end{array}$ & $\begin{array}{l}\text { Plastic } \\
(\%)\end{array}$ \\
\hline \multicolumn{5}{|c|}{ 1. Infiltration /Exfiltration Module } \\
\hline Pipe Age & 15 & 15 & 15 & 15 \\
\hline Groundwater Table & 35 & 35 & 35 & 35 \\
\hline Soil Type & 20 & 20 & 20 & 20 \\
\hline Pipe Surcharging & 30 & 30 & 30 & 30 \\
\hline \multicolumn{5}{|l|}{ 2. Blockage Module } \\
\hline Pipe Age & 10 & 10 & 10 & 10 \\
\hline Pipe Length & 5 & 5 & 5 & 5 \\
\hline Wall thickness & 10 & 10 & 5 & 5 \\
\hline Pipe Diameter & 20 & 20 & 20 & 20 \\
\hline Pipe Slope & 20 & 20 & 25 & 25 \\
\hline Flow Velocity & 10 & 10 & 10 & 10 \\
\hline Flow depth/Diameter (d/D) & 5 & 5 & 5 & 5 \\
\hline Density of Connection & 5 & 5 & 5 & 5 \\
\hline Maintenance Frequency & 15 & 15 & 15 & 15 \\
\hline \multicolumn{5}{|l|}{ 3. Root Penetration Module } \\
\hline Pipe Age & 25 & 25 & 25 & 25 \\
\hline Pipe Wall Thickness & 5 & 5 & 5 & 5 \\
\hline Pipe Diameter & 20 & 20 & 20 & 20 \\
\hline Proximity to trees & 30 & 30 & 30 & 30 \\
\hline Maintenance Frequency & 20 & 20 & 20 & 20 \\
\hline \multicolumn{5}{|c|}{ 4. Hydraulic (Capacity) Module } \\
\hline Flow depth/Diameter (d/D) & 20 & 20 & 20 & 20 \\
\hline Flow Velocity & 10 & 10 & 10 & 10 \\
\hline Pipe Slope & 20 & 20 & 20 & 20 \\
\hline
\end{tabular}




\begin{tabular}{|l|r|l|l|l|}
\hline \multicolumn{1}{|c|}{ Parameters } & $\begin{array}{l}\text { Concrete } \\
(\boldsymbol{\%})\end{array}$ & $\begin{array}{l}\text { Clay } \\
(\boldsymbol{\%})\end{array}$ & $\begin{array}{l}\text { Metal } \\
(\boldsymbol{\%})\end{array}$ & $\begin{array}{l}\text { Plastic } \\
(\boldsymbol{\%})\end{array}$ \\
\hline Pipe Surcharging & 25 & 25 & 25 & 25 \\
\hline Maintenance Frequency & 25 & 25 & 25 & 25 \\
\hline
\end{tabular}

\subsection{Fuzzy Inference Performance Index Evaluation Model (F-PIE)}

The F-PIE model is an update of the W-PIE model. It evaluates wastewater pipe based on 8 performance modules including External Corrosion, Internal Corrosion, Surface Wear, Integrity, Infiltration/Exfiltration, Blockage, Root Penetration, and Hydraulic (Capacity) similar to W-PIE. It is based on a fuzzy inference system built in MATLAB. F-PIE consists of two main parts, membership functions, and if-then fuzzy rules.

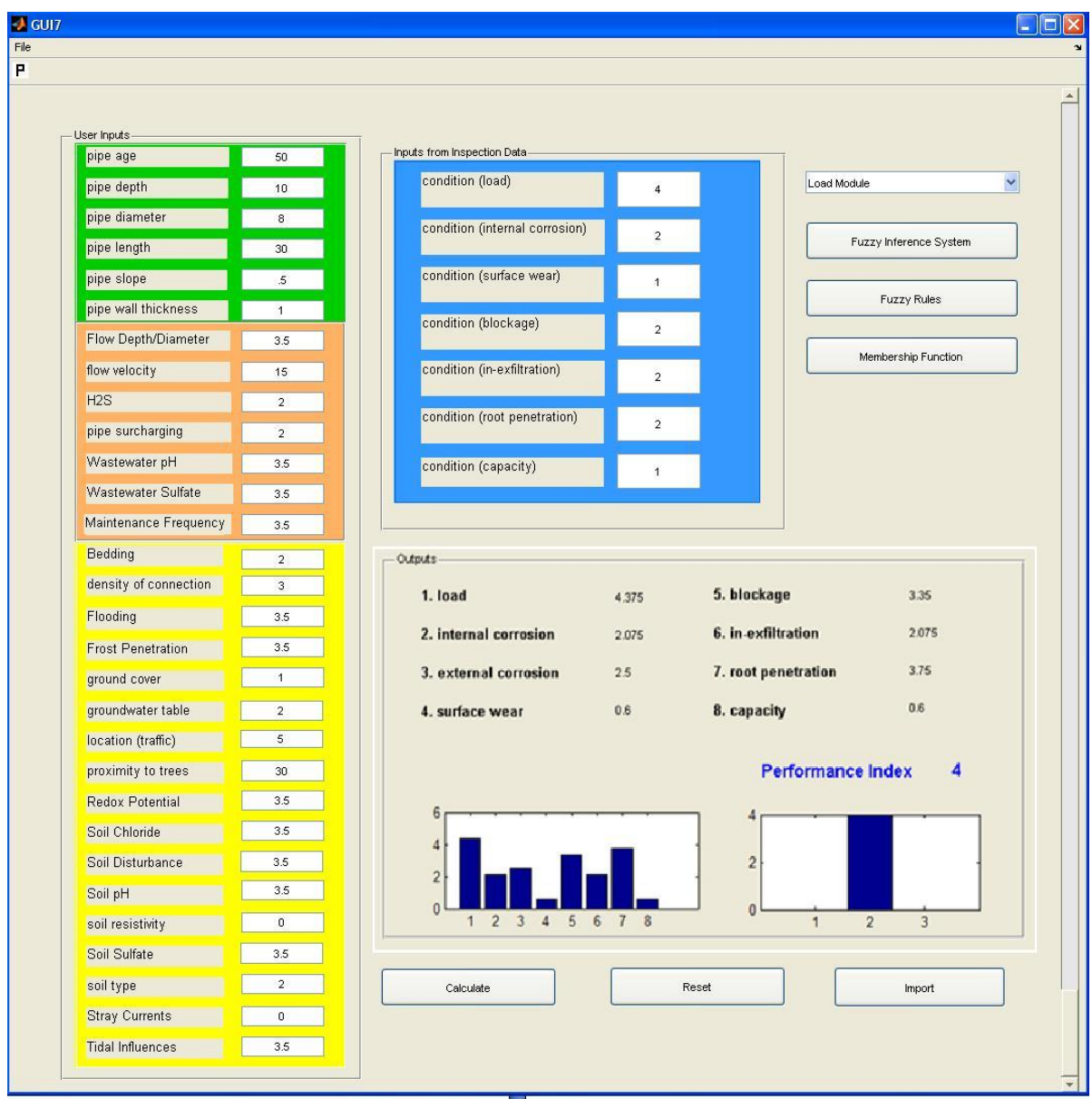

Figure 4.4 F-PIE User Interface 
The membership functions and rules used in the model were set up based on prior research knowledge and engineering judgment. The membership functions and rules apply linguistic expressions representing relationships and uncertainty of data. The membership functions and rules are subjective and the same in applying this model to various utilities. However, inconsistency may occur under circumstances and a change in the membership and rule would be necessary to match each utility's characteristics.

Most of the input units are direct, for example, pipe age in years and pipe length in feet as used in W-PIE. But some units are in grading scales (0-5), such as the bedding condition and pipe condition. Figure 4.4 shows the F-PIE user interface which consists of user input parameter, inspection data, and output spaces. Each parameter has its own membership function presenting linguistic expression and degree of membership. Fuzzy if-then rules are applied in the calculation. Unlike W-PIE, F-PIE can take dependency of parameters into account and has multiple effects to a system. In wastewater pipe performance case, for example, if the pipe condition and bedding condition are poor, the structure failure is likely possible.

\section{Development of Membership functions}

Each parameter has its own membership function presenting linguistic expression and degree of membership. An example of membership functions is shown in Figure 4.5.

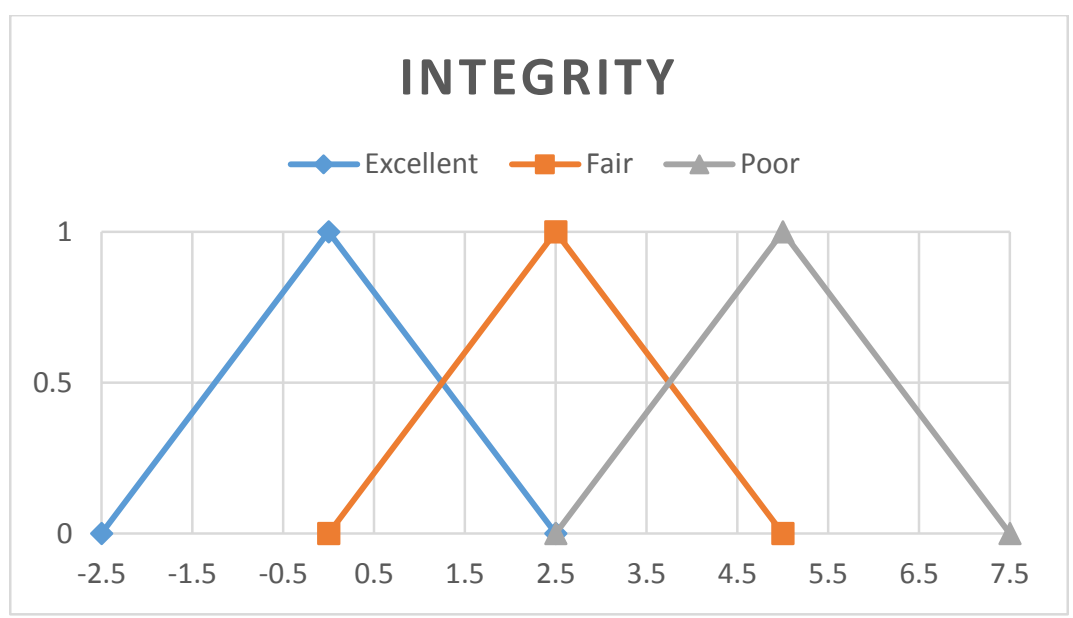

Figure 4.5 Example of Membership Functions 
The development of membership functions is limited to expert-driven methods due to incomplete and unavailability of wastewater pipe data rather than data-driven methods. All membership functions can be found in Appendix D.

\section{Development of If-Then Rule Statements}

Fuzzy if-then rules were used in representing the knowledgebase in the F-PIE. The rule based expert system is developed on the heuristic expert-driven technique. Each fuzzy if-then rule presented in this section reflects on the general rule of thumb and expert knowledge acquired from various professionals including engineers and managers from different utilities across the U.S, consultants, pipe associations and condition assessment companies. The experts were interviewed and the parameters affecting each module were identified. The rules were created and were sent to experts for review and validation. The rules associated with the performance model are presented in appendix E. Table 4.8 to Table 4.15 show membership ranges and linguistic terms for each parameter. The parameter ranges are organized by module.

Table 4.8 Fuzzy Inference Model Membership Ranges (Integrity)

\begin{tabular}{|c|c|c|c|}
\hline \multirow{15}{*}{ 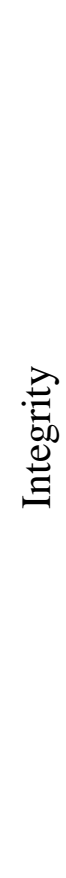 } & Input Variables & Membership Function Ranges & Linguistic Terms \\
\hline & Condition & $(0-2.5),(0.5-4.5),(2.5-5)$ & Good, Fair, Poor \\
\hline & Location & $(0-2),(0.5-4.5),(3-5)$ & Good, Fair, Poor \\
\hline & Soil Type & $(0-2),(0.5-4.5),(3-5)$ & Best, Average, Worst \\
\hline & Pipe Depth & $(0-8),(2-18),(12-20)$ & Shallow, Average, Deep \\
\hline & Groundwater Table & $(0-1),(0.5-4.5),(3-5)$ & $\begin{array}{l}\text { Below Pipe, Close to Pipe, } \\
\text { Above Pipe }\end{array}$ \\
\hline & Bedding Condition & $(0-2),(0.5-4.5),(3-5)$ & Good, Fair, Poor \\
\hline & Ground Cover & $(0-2),(0.5-4.5),(3-5)$ & Best, Average, Worst \\
\hline & Pipe Age & $(0-30),(20-60),(40-100)$ & New, Average, Old \\
\hline & Pipe Surcharging & $(0-2),(0.5-4.5),(3-5)$ & low, Moderate, High \\
\hline & Soil Disturbance & No, yes & Undesirable, Desirable \\
\hline & Flooding & No, Yes & Undesirable, Desirable \\
\hline & Frost Penetration & No, Yes & Undesirable, Desirable \\
\hline & \begin{tabular}{|l|} 
Output Variables \\
\end{tabular} & Membership Function Ranges & Linguistic Terms \\
\hline & \begin{tabular}{|l} 
Integrity \\
\end{tabular} & $(-2.5-2.5),(0-5),(2.5-7.5)$ & Excellent, Fair, Poor \\
\hline
\end{tabular}


Table 4.9 Fuzzy Inference Model Membership Ranges (Internal Corrosion)

\begin{tabular}{|c|c|c|c|}
\hline \multirow{12}{*}{ 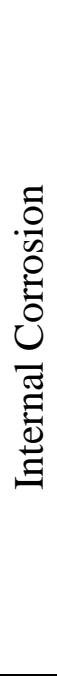 } & Input Variables & Membership Function Ranges & Linguistic Terms \\
\hline & Condition & $(0-2.5),(0.5-4.5),(2.5-5)$ & Good, Fair, Poor \\
\hline & Pipe Age & $(0-30),(20-60),(40-100)$ & New, Average, Old \\
\hline & Pipe Slope & $(0-2),(1.5-4.5),(4-5)$ & low, Moderate, High \\
\hline & Flow Velocity & $(2-3.5),(2.5-8),(7-10)$ & low, Moderate, High \\
\hline & $\mathrm{H} 2 \mathrm{~S}$ & $(0-50),(0-150),(100-500)$ & Low, Moderate, High \\
\hline & Flow Depth/Diameter & $(0-0.3),(0.2-0.8),(0.7-1.0)$ & Empty, half, Full \\
\hline & Wastewater pH & $(0-6.5),(6-9.5),(9-14)$ & Acid, Neutral, Base \\
\hline & Wastewater Sulfate & $(100-2,200),(150-300)$ & low, High \\
\hline & Maintenance Frequency & $(0-2),(1-4),(3-5)$ & Often, Regular, Rarely \\
\hline & \begin{tabular}{|l|} 
Output Variables \\
\end{tabular} & Membership Function Ranges & Linguistic Terms \\
\hline & Internal Corrosion & $(-2.5-2.5),(0-5),(2.5-7.5)$ & Low, Moderate, High \\
\hline
\end{tabular}

Table 4.10 Fuzzy Inference Model Membership Ranges (External Corrosion)

\begin{tabular}{|c|c|c|c|}
\hline \multirow{12}{*}{ 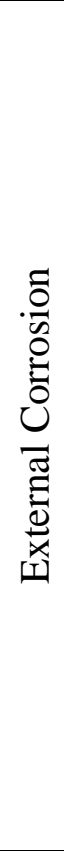 } & Input Variables & Membership Function Ranges & Linguistic Terms \\
\hline & Pipe Age & $(0-30),(20-60),(40-100)$ Years & New, Average, Old \\
\hline & $\begin{array}{l}\text { Pipe Wall } \\
\text { Thickness }\end{array}$ & $(0-10),(6-24),(20-30)$ & $\begin{array}{l}\text { Low Loss, Moderate Loss, } \\
\text { High Loss }\end{array}$ \\
\hline & Groundwater Table & $(0-1),(0.5-4.5),(3-5)$ & $\begin{array}{l}\text { Below Pipe, Close to Pipe, } \\
\text { Above Pipe }\end{array}$ \\
\hline & Soil Resistivity & $\begin{array}{l}(0-4,000),(1,000-9,000), \\
(6,000-10,000)\end{array}$ & Low, Moderate, High \\
\hline & Soil pH & $(0-6.5),(6-9.5),(9-14)$ & Acid, Neutral, Base \\
\hline & Soil Sulfate & $\begin{array}{l}(1,000-2,500),(1,500-4,500), \\
(3,500-5,000)\end{array}$ & low, Moderate, High \\
\hline & Soil Chloride & $(200-350),(300-400)$ & Low, High \\
\hline & Redox Potential & $(-100$ to -60$),(-80$ to -20$),(-50$ to 0$)$ & High, Moderate, Low \\
\hline & Stray Currents & No, Yes & Undesirable, Desirable \\
\hline & Output Variables & Membership Function Ranges & Linguistic Terms \\
\hline & External Corrosion & $(-2.5-2.5),(0-5),(2.5-7.5)$ & Low, Moderate, High \\
\hline
\end{tabular}


Table 4.11 Fuzzy Inference Model Membership Ranges (Surface Wear)

\begin{tabular}{|c|c|c|c|}
\hline \multirow{8}{*}{ 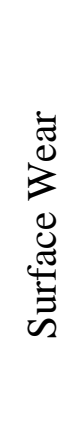 } & \begin{tabular}{|l} 
Input Variables \\
\end{tabular} & Membership Function Ranges & Linguistic Terms \\
\hline & Condition & $(0-2.5),(0.5-4.5),(2.5-5)$ & Good, Fair, Poor \\
\hline & Pipe Age & $(0-30),(20-60),(40-100)$ & New, Average, Old \\
\hline & Flow Velocity & $(2-3.5),(2.5-8),(7-10)$ & low, Moderate, High \\
\hline & \begin{tabular}{|l|} 
Pipe Slope \\
\end{tabular} & $(0-2),(1.5-4.5),(4-5)$ & low, Moderate, High \\
\hline & Type of Cleaning & $(0-2),(1-4),(3-5)$ & Often, Regular, Rarely \\
\hline & \begin{tabular}{|l} 
Output Variables \\
\end{tabular} & Membership Function Ranges & Linguistic Terms \\
\hline & Surface Wear & $(-2.5-2.5),(0-5),(2.5-7.5)$ & Low, Moderate, High \\
\hline
\end{tabular}

Table 4.12 Fuzzy Inference Model Membership Ranges (Blockage)

\begin{tabular}{|c|c|c|c|}
\hline \multirow{12}{*}{ 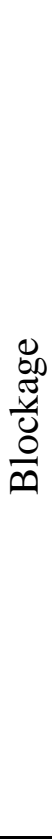 } & Input Variables & $\begin{array}{l}\text { Membership Function } \\
\text { Ranges }\end{array}$ & Linguistic Terms \\
\hline & Condition & $(0-2.5),(0.5-4.5),(2.5-5)$ & Good, Fair, Poor \\
\hline & Pipe Age & $(0-30),(20-60),(40-100)$ & New, Average, Old \\
\hline & Pipe Length & $(0-200),(100-400),(300-500)$ & Short, Average, Long \\
\hline & Pipe Diameter & $(0-24),(12-60),(36-72)$ & Small, Moderate, Large \\
\hline & Pipe Slope & $(0-2),(1.5-4.5),(4-5)$ & low, Moderate, High \\
\hline & Flow Velocity & $(0-3.5),(0.5-8),(7-10)$ & low, Moderate, High \\
\hline & Flow Depth/Diameter & $(0-0.3),(0.2-0.8),(0.7-1.0)$ & Empty, half, Full \\
\hline & Density of Connections & $(0-2),(1-4),(3-5)$ & $\begin{array}{l}\text { Light, Moderate, Very } \\
\text { Dense }\end{array}$ \\
\hline & $\begin{array}{l}\text { Maintenance } \\
\text { Frequency }\end{array}$ & $(0-2),(1-4),(3-5)$ & Often, Regular, Rarely \\
\hline & Output Variables & $\begin{array}{l}\text { Membership Function } \\
\text { Ranges }\end{array}$ & Linguistic Terms \\
\hline & Blockage & $(-2.5-2.5),(0-5),(2.5-7.5)$ & Low, Moderate, High \\
\hline
\end{tabular}


Table 4.13 Fuzzy Inference Model Membership Ranges (In-exfiltration)

\begin{tabular}{|c|c|c|c|}
\hline \multirow{8}{*}{ 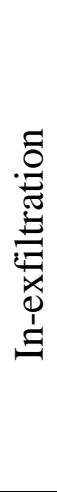 } & Input Variables & Membership Function Ranges & Linguistic Terms \\
\hline & Condition & $(0-2.5),(0.5-4.5),(2.5-5)$ & Good, Fair, Poor \\
\hline & Pipe Age & $(0-30),(20-60),(40-100)$ & New, Average, Old \\
\hline & Pipe Surcharging & $(0-2),(0.5-4.5),(3-5)$ & low, Moderate, High \\
\hline & Groundwater Table & $(0-1),(0.5-4.5),(3-5)$ & $\begin{array}{l}\text { Below Pipe, Close to Pipe, } \\
\text { Above Pipe }\end{array}$ \\
\hline & Soil Type & $(0-2),(0.5-4.5),(3-5)$ & Best, Average, Worst \\
\hline & Output Variables & Membership Function Ranges & Linguistic Terms \\
\hline & In-exfiltration & $(-2.5-2.5),(0-5),(2.5-7.5)$ & Low, Moderate, High \\
\hline
\end{tabular}

Table 4.14 Fuzzy Inference Model Membership Ranges (Root Intrusion)

\begin{tabular}{|c|c|c|c|}
\hline \multirow{8}{*}{ 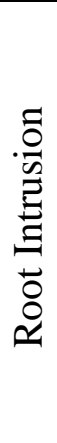 } & Input Variables & Membership Function Ranges & Linguistic Terms \\
\hline & Condition & $(0-2.5),(0.5-4.5),(2.5-5)$ & Good, Fair, Poor \\
\hline & Pipe Age & $(0-30),(20-60),(40-100)$ & New, Average, Old \\
\hline & Pipe Diameter & $(0-24),(12-60),(36-72)$ & Small, Moderate, Large \\
\hline & Proximity to Trees & $(0-10),(2-18),(10-20)$ & low, Moderate, High \\
\hline & Maintenance Frequency & $(0-2),(1-4),(3-5)$ & Often, Regular, Rarely \\
\hline & Output Variables & Membership Function Ranges & Linguistic Terms \\
\hline & Root Intrusion & $(-2.5-2.5),(0-5),(2.5-7.5)$ & Low, Moderate, High \\
\hline
\end{tabular}

Table 4-15 Fuzzy Inference Model Membership Ranges (Capacity)

\begin{tabular}{|c|c|c|c|}
\hline \multirow{11}{*}{ 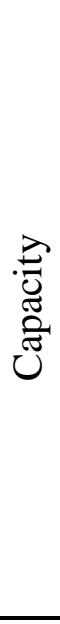 } & Input Variables & Membership Function Ranges & Linguistic Terms \\
\hline & Condition & $(0-2.5),(0.5-4.5),(2.5-5)$ & Good, Fair, Poor \\
\hline & Pipe Slope & $(0-2),(1.5-4.5),(4-5)$ & low, Moderate, High \\
\hline & Flow Depth/Diameter & $(0-0.3),(0.2-0.8),(0.7-1.0)$ & Empty, half, Full \\
\hline & Flow Velocity & $(2-3.5),(2.5-8),(7-10)$ & low, Moderate, High \\
\hline & Pipe Surcharging & $(0-2),(0.5-4.5),(3-5)$ & low, Moderate, High \\
\hline & Maintenance Frequency & $(0-2),(1-4),(3-5)$ & Often, Regular, Rarely \\
\hline & Flooding & No, Yes & Undesirable, Desirable \\
\hline & Tidal Influence & No, Yes & Undesirable, Desirable \\
\hline & Output Variables & Membership Function Ranges & Linguistic Terms \\
\hline & Capacity & $(-2.5-2.5),(0-5),(2.5-7.5)$ & Low, Moderate, High \\
\hline
\end{tabular}


In this chapter, we presented two performance index models, weighted factor and fuzzy inference. The performance index results utilizing real utility data from different cities are presented in the next chapter. Evaluation of the developed performance index comprises of analyzing the data on different approaches, through case studies and result comparisons. 


\section{CHAPTER 5}

\section{Wastewater Pipe Performance Index Evaluation}

Researchers have proposed different models in order to predict the condition/performance and failure of wastewater pipelines. However, the question still remains unanswered on whether the model is predicting a reasonable value in regard to the actual value found in the field. This is answered by the process of model verification and validation. In this research verification and validation of a fuzzy logic performance model for wastewater pipelines is discussed. Model validation can be defined as "ensuring that the computer program of the computerized model and its implementation are correct" (Sargent, 2007), also model validation can be defined as "substantiation that a computerized model within its domain of applicability possesses a satisfactory range of accuracy consistent with the intended application of the model" (Schlesinger, 1979). In this chapter, model validation is done by evaluating the relation of the input parameters with the model output.

Papers that have previously presented model verification and validation processes are discussed. Khan et al. (2010) describes a model which uses artificial neural networks to investigate the importance and impact of certain characteristics of sewer pipes upon their structural performance, expressed in terms of condition rating. In this paper the authors have also described the process to validate the model by mathematical diagnosis. They tested their model with real 
data which included parameters like pipe dimensions, pipe age, concrete class, for example. The validation process was divided into three sets namely training data test data and production data. The authors used the first two sets to develop the models and the production data to test the performance of the trained network. Four indicators were used as the measure of goodness of the model namely, average invalidity and validity percentages, coefficient of determination, root mean square error, and mean absolute error function. The authors also performed a sensitivity analysis of their six input factors in which they varied one parameter at a time by keeping others constant and observed the results.

Golroo et al. (2012) describes the validation of Pervious Concrete Pavement (PCP) condition through panel rating method. They validated their model by checking the panel rating data on whether or not there was any significant difference among the PCP sections. The authors used a statistical tool ANOVA (Analysis of variance) that provides tests (i.e., t-test and F-test) that showed whether or not the mean and variance of several series of numbers (subjective or objective) are equal at a specific level of significance. They also used an in situ inspection technique in which they statistically evaluated the expected value of the ratings for the validation of the surface distress ratings with the coefficient of determination.

Sargent (2007) discusses various validation techniques and tests used in model verification and validation. These techniques include comparison to other models where various results (e.g., outputs) of the simulation model being validated are compared to results of other (valid) models; Extreme Condition Tests where the model structure and outputs should be possible for any extreme and unlikely combination of levels of factors in the system; Internal Validity: Several replication (runs) of a stochastic model are made to determine the amount of (internal) stochastic variability in the model; Face Validity which comprises of asking individuals knowledgeable about the system whether the model and/or its behavior are reasonable i.e. is the logic in the conceptual model correct and are the model's input-output relationships reasonable; Parameter Variability or Sensitivity Analysis which consists of changing the values of the input and internal parameters of a model to determine the effect upon the model's behavior or output. 
Pace (2004) defines and describes verification and validation. According to the author verification has two aspects: design i.e. whether or not all specifications and nothing else that are included in the model or simulation design, and implementation i.e. whether or not all specifications and nothing else are included in the model or simulation as built. And validation has also two aspects according to the author: conceptual validation (when the anticipated fidelity of the model or simulation conceptual model is assessed) and results validation (when results from the implemented model or simulation are compared with an appropriate referent to demonstrate that the model or simulation can in fact support the intended use). This paper also identifies and describes key modeling and simulation verification and validation challenges and indicates how they are being addressed. Critical challenges include multi-resolution modeling, interoperability, visualization, behavioral modeling, security, confidence assessment, visualization in architectural engineering environments and integration of modeling and simulation (M\&S) into training and education were discussed in the paper.

Sargent and Pace have described the concept of verification and validation in general. Khan et al. and Golroo et al. used statistical methods and sensitivity analysis which performs well when only a few parameters are taken into consideration in a model. Another method is in need when a large number of parameters are considered within a model so that the behavior of the input parameters as a whole is captured.

In this chapter, the performance index presented in chapter 4 were evaluated based on 2 different approaches, artificial data and field data. In the first approach the model is evaluated utilizing artificial input data. Specifically, model is analyzed by evaluating three bands of input values classifying the best, moderate and worst case values. The purpose of testing the model with these three band is to observe the behavior of the model under known inputs. The model shall give expected results for the known inputs in order to be verified. For example, if the model inputs were in the best band, the model results should reflect that the evaluated asset should be in excellent condition.

The second approach is the testing with the field data. An actual field dataset representing the variables from an asset network in use shall be utilized in order to observe the behavior of the 
model. The purpose of this approach is to eliminate any discrepancies and inaccuracies that can be spotted with the actual field data. Figure 5.1 represents evaluation framework.

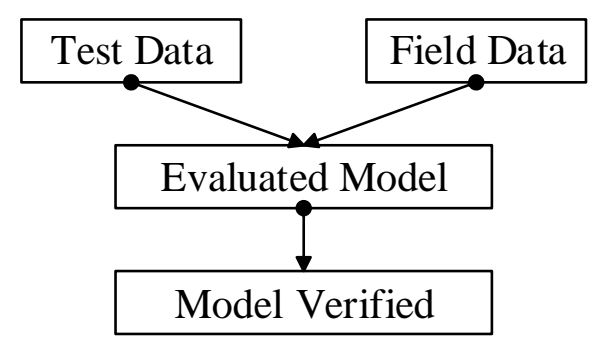

\section{Figure 5.1 Evaluation Methodology Framework}

\subsection{Artificial Data Evaluation}

Initial testing of the model began by evaluating several tests or "imaginary" input values which assessed the behavior of the model. The input values were provided in three bands which evaluated the best, moderate and worst case scenarios. Three bands were chosen because of the fact that the output value of the model ranges from 0 (Excellent) to 5 (Very Poor/Failed). Moreover we know that when the worst values are entered the output must be the worst, similar for the best and moderate values. Overall, these bands ensure that the output fell within that desirable range. In each band parameters were changed to observe how individual parameters would affect the outcome. Several points were observed during test data validation which is summarized below.

- The model performed well when analyzing input data by the three band scenarios. When the best input parameter values were entered in the model the output reflected with the best condition ranges. Similar results were found for the other two bands.

- It was observed there was large weight on the CCTV data which is evident from the fact that even when all the parameters have worst value but CCTV data has the best value the model give the moderate values.

- The value of external corrosion module is determined solely by the input parameters without taking CCTV data into consideration. The final Performance index is determined by the least favorable condition of the output modules. 
- The effect of CCTV data on the model was independent on each of the modules. For example if we change the value of blockage in CCTV data, it will only affect the result of blockage module and will not affect the others.

- Overall the model behaved very well. The output values changed according to the fuzzy logic rules and they were only affected by the input variables they were related to, not by the variables of other modules.

- In the best case scenarios, the overall performance gives resultant values close to or exactly 0 "Excellent” performance.

- In the worst case scenarios, the overall performance gives resultant values close to or exactly 5 "Very Poor" performance.

\subsection{Field Data Evaluation}

The first step of the process is to determine the real condition of the pipe samples evaluated. This real condition is addressed as "ground truth". The ground truth is a crucial value to compare the model outputs and determine if the model is accurately representing the behavior of the real system. This value can be determined by conducting laboratory tests or other condition assessment techniques to assess the actual condition of an asset

The heuristic approach is the second approach to be followed to evaluate the models. This approach relies on the condition rating received from the field experts. This rating is determined on the visual inspection of the assets or by evaluating the variables by the field engineers or other professionals that have some experience on the pipe deterioration. The purpose of this approach is to employ the experience of the field experts in the evaluation process.

The outputs determined by following these two approaches are directly compared with the results of the model while using the same input variables. These three results shall not be statistical different in order to conclude that the model is validated. In case where the results are different, the model shall be further calibrated. The framework to evaluate the field data is summarized in Figure 5.2. 


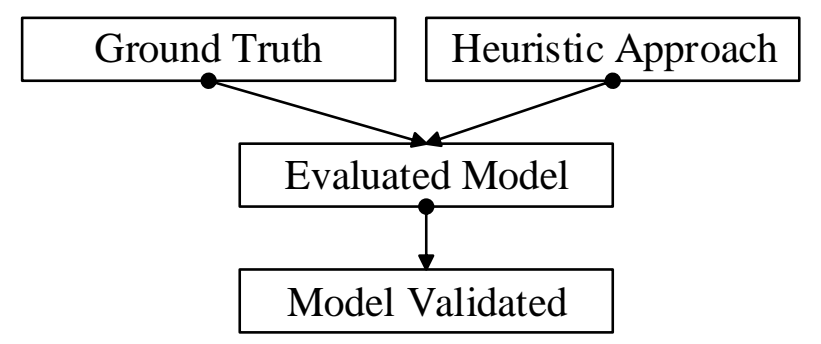

Figure 5.2 Validation Framework

Testing of the wastewater pipes samples in a laboratory setting was not possible at the time. However, there are condition assessment techniques to capture the ground truth of the wastewater pipes. These condition assessment techniques consist of evaluating the wastewater pipes using a Closed Circuit Television (CCTV) camera that travels inside and captures the pictures of defects in the pipelines. These pictures are then evaluated by field experts to assign a condition index to these evaluated assets. The PACP defect rating system is used to quantify the field observation with digital numbers. The grade acquired by the system can be considered both as the ground truth and the expert opinion to compare and evaluate the model.

\subsection{Case Studies}

Case studies utilizing real utility data were facilitated. The case studies consisted of discussions of the utility data and evaluations of the performance models. A memorandum of understanding (MOU) was signed with all participating utilities. Meetings were conducted to facilitate the data transfer process, obtain crucial feedback on the performance index, and to discuss the current state of pipe infrastructure data management in each community. The data used in these case studies have been manipulated for security reasons. Overall, 11 utilities participated in this research;

1. Seattle Public Utilities Seattle, Seattle, WA

2. Western Virginia Water Authority, Roanoke, VA

3. Orange County Sanitation District, Orange County, CA

4. Pittsburgh Water and Sewer Authority, Pittsburgh, PA 
5. Massachusetts Water Resources Authority, Boston, MA

6. Town of Blacksburg, Blacksburg, VA

7. Atlanta Public Utilities, Atlanta, GA

8. Cobb County Water System, Cobb County, GA

9. Washington Suburban Sanitary Commission, Washington, DC

10. Aurora Water, Aurora, CO

11. City of Anchorage, Anchorage, AK

These utilities were selected by size and geographical location. The size of the utilities ranges from major cities to smaller towns. They are located from east to west coast to represent utilities in the U.S. as shown in figure 5.3.

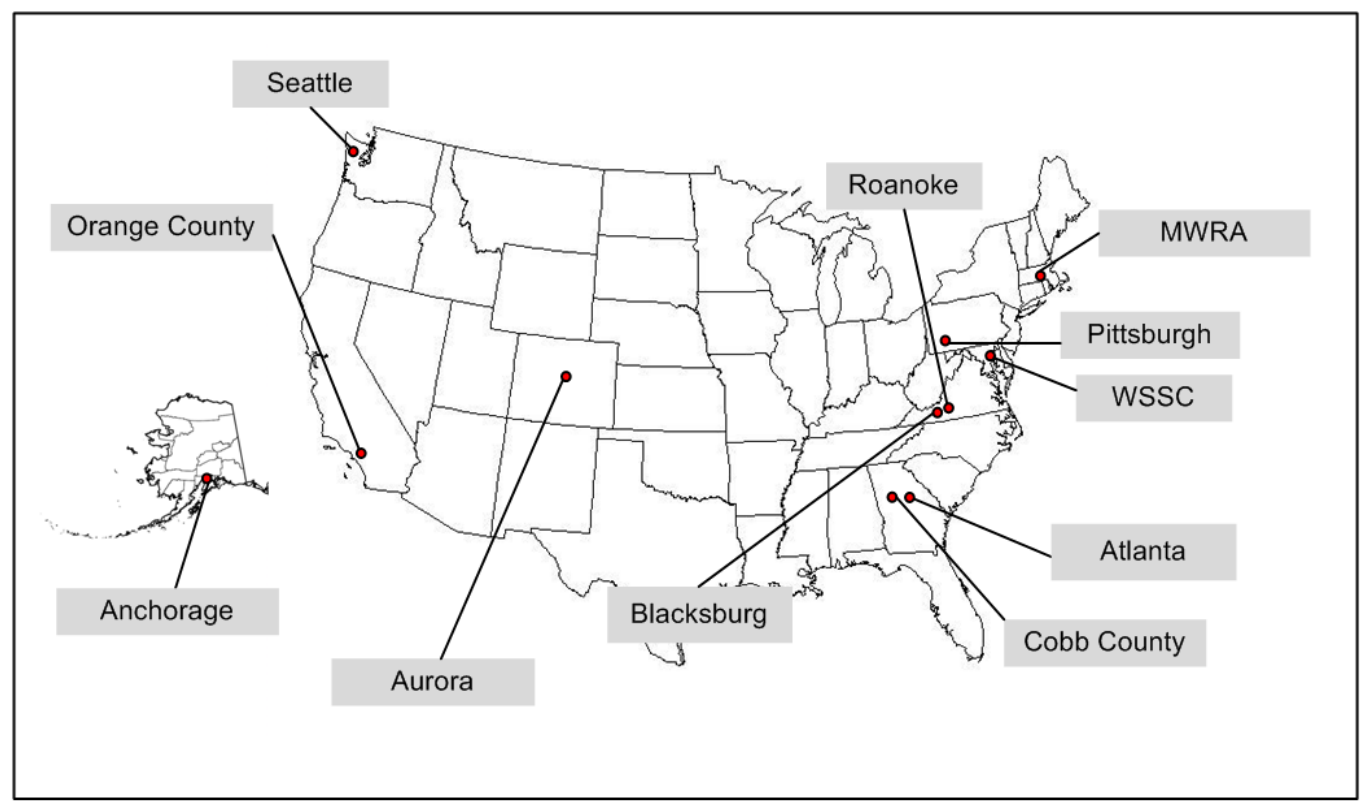

Figure 5.3 Target Utilities for Pilot Study of the Model

These 11 utilities were visited to assist in research development. During each visit, wastewater pipe data, CCTV data, and other related data that was used as input in piloting the performance models were obtained. Data acquisition was a very extensive process considering many different sources, formats, and modeling. Overview of the participating utilities and utility data from 11 utilities are presented in Appendix F. During the development process, we presented 
the performance index and received comments on methodology, parameters, and parameter ranges on both weighted factor and fuzzy inference models. We were trying to capture expert knowledge on wastewater pipes, for instance, how pipes fail, what parameters play a major role, and current practices on condition assessment; how the utility evaluates their pipe conditions, what standard is used. Utility staffs, engineers, and managers from listed utilities provided critical engineering knowledge in relation to parameters and their effect on pipe performance as seen in the field.

In this chapter, case study of three cities; City B, City H, and Washington Suburban Sanitary Commission WSSC are presented. All other case studies can be found in Appendix G.

\section{Case Study: City $B$}

We analyzed the data received from City B and evaluated each pipe section (ManholeManhole) using performance models, including W-PIE and F-PIE. City B provided shape-files, geo-guide, and CCTV Excel files. Additional data were provided via an FTP site. Altogether, there were 20 shape-files and PACP rating indexes from 2005 to 2010. City B also provided a geo-guide, which is a metadata file that describes all the attributes mentioned in the shape-files. The data provided by City B included 15 parameters that were used in this pilot study.

\section{Parameters}

- Pipe age

- Pipe diameter

- Pipe depth

- Pipe slope

- Pipe length

- Pipe material

- Traffic

- Soil type

- Soil corrosivity

- Unstable soil/ soil disturbance

- Flooding 
- Groundcover

- Closeness to trees

- Number of connections

- Pipe condition (PACP rating index)

Pipe age, pipe diameter, pipe depth, pipe slope, pipe length, and pipe material were stored as attributes in the mainline GIS shape-file. Traffic data were derived from the arterial shape-file, and a spatial analysis tool was used to approximate the distances from the pavement to the pipes. Soil data were provided in separate shape-files. The soil testing shape-file contained soil type and soil corrosivity. The unstable-slope shape-file lists the areas in which there are unstable soils due to the steep slope. The number of connections was calculated by counting the lateral pipes in a lateral shape-file that intersects with a pipe of interest. City B has used PACP coding since 2005. Previously, a customized coding system, developed in-house, had been used since the late1960s. There were still some past inspection data that must be converted into digital format. For this reason, we were able to access only the pipe ratings after 2005. Figure 6-3 presents the PACP rating index for City B. The pipes were rated based on the scale (0-5) where 0 (green) represents the pipe in excellent condition, and 5 (red) represents failed condition. The pipes for which there are no inspection data (0), which are indicated in black, were assumed to be excellent for the purpose of calculation. Based on the data received, models using both W-PIE and F-PIE were run, and the results are shown below.

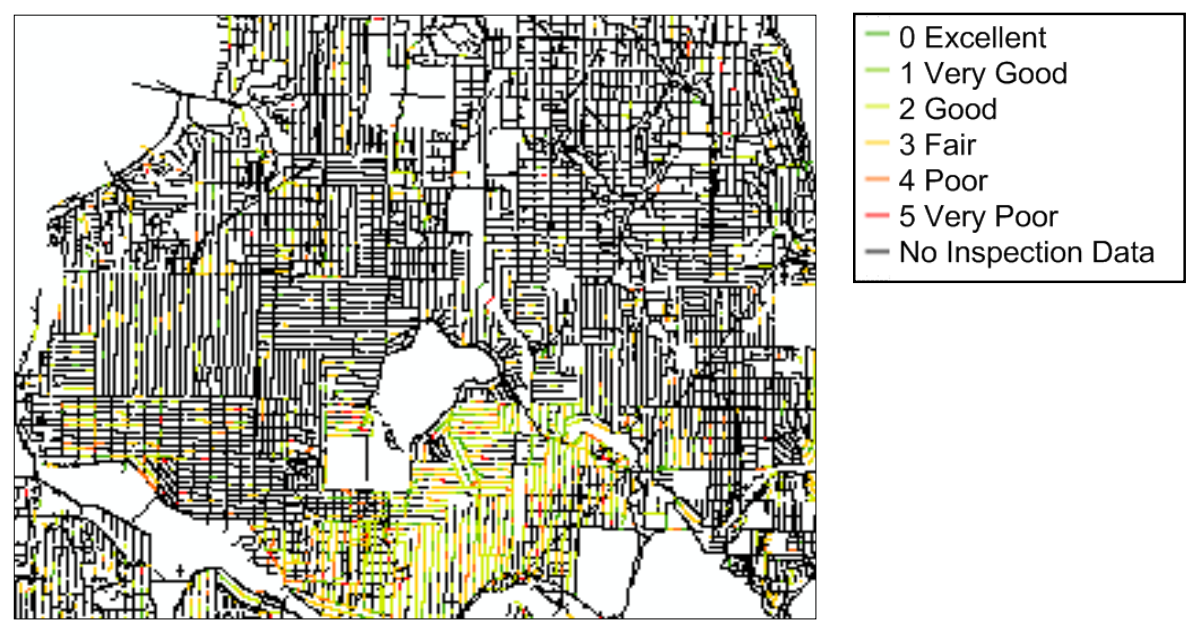

Figure 5.4 PACP Rating Index 


\section{- W-PIE AND F-PIE Results}

Figure 5.4 shows the results of the performance index calculated by the weighted-factor model, where 0 indicates excellent pipes, and 5 indicates very poor pipes. The results are mapped to GIS to illustrate the distribution of the performance index. As shown in Figure 5.4, the majority of the performance index of City B was rated as 0 and 1, which indicate excellent and good, respectively. From the results presented here, the failed and poor pipes are aggregated in of the center of the city. Further investigation is needed to understand the causes of failure. Figure 5.5 is the map of the performance index obtained by F-PIE. The result is color coded, with green representing excellent pipes, and red representing failed pipes.

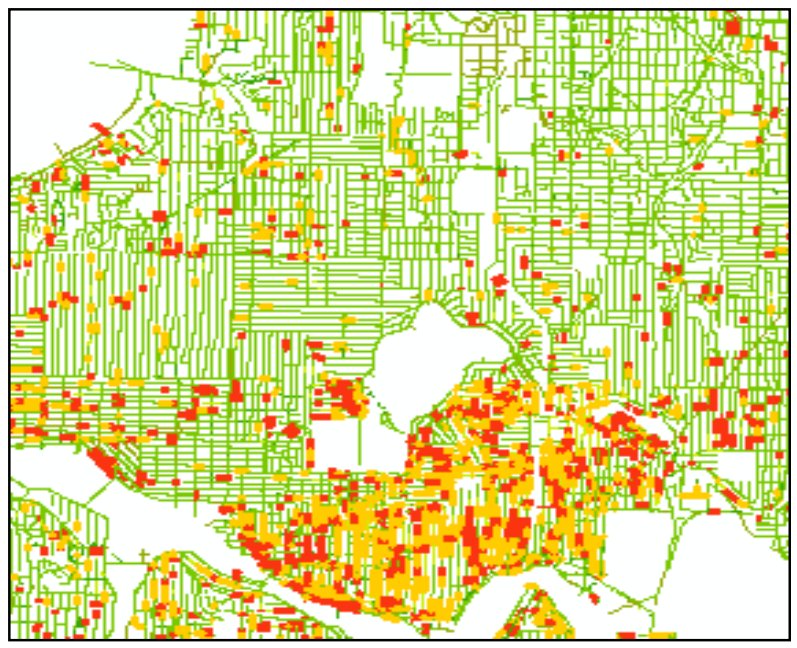

-0 Excellent
-1 Very Good
-2 Good
-3 Fair
-4 Poor
-5 Very Poor
- No Inspection Data

Figure 5.5 Performance Index by W-PIE

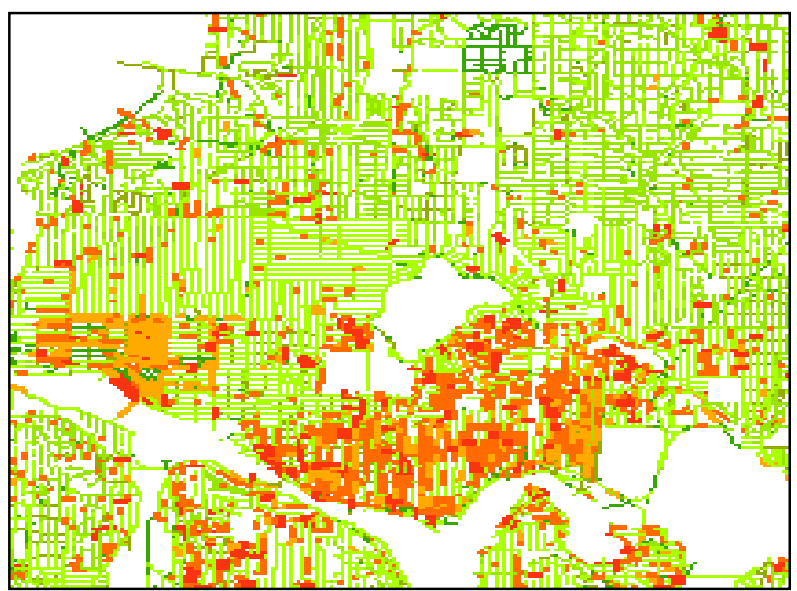

-0 Excellent
-1 Very Good
-2 Good
-3 Fair
-4 Poor
-5 Very Poor
- No Inspection Data

Figure 5.6 Performance Index by F-PIE 
The results show that most of the pipes were rated 0,1 , and 2. Most of the poor pipes were aggregated in the middle part of the city. By comparing Figure 5.4 to Figure 5.5, it was evident that there are more poor pipes in the results provided by W-PIE than the PACP index because the knowledge base introduced more parameters to the calculation. From the results shown in Figures 5.4 to 5.6, the results from F-PIE show more poor pipes than the results from W-PIE and PACP. The F-PIE provide more sensitive results, whereas the weighted-factor model is less sensitive because the weights assigned to each parameter are fixed and distributed throughout all parameters in the module.

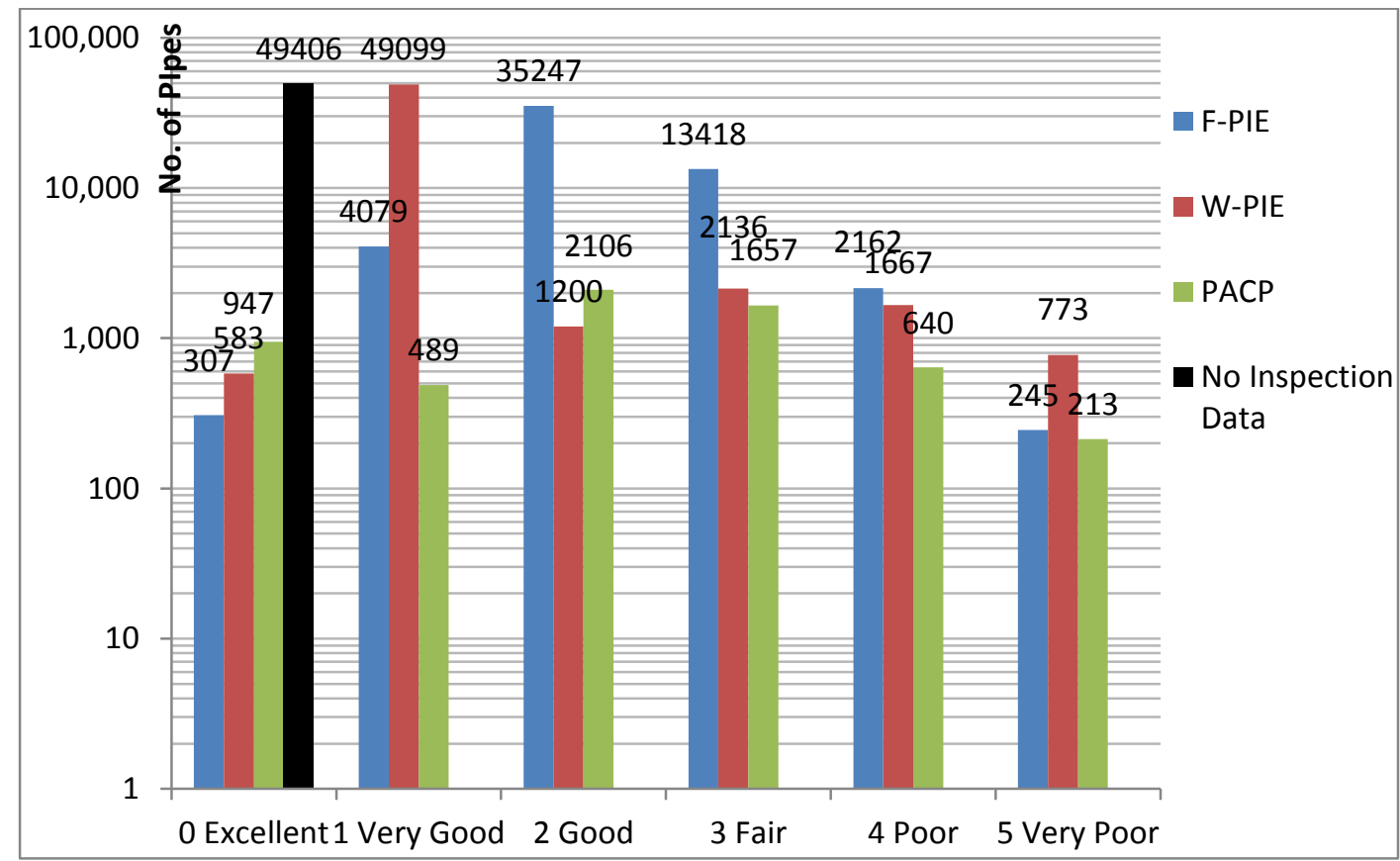

Figure 5.7 Performance Index by No PACP Data, PACP, F-PIE, and W-PIE

Figure 5.7 shows the bar chart of the performance index calculated by No-PACP, PACP, W-PIE, and F-PIE to illustrate the distribution of the results, where the $\mathrm{x}$-axis is the performance index, and the y-axis is the number of pipes that fall within each value. Compared to PACP, the results from W-PIE can be different for the next two higher values or stay at the same value; if the PACP index is 1, the W-PIE index may be 1 (very good), 2 ( good), or 3( fair), but not 0 . A similar situation occurred with F-PIE, but the F-PIE results were more sensitive than those of WPIE, and we see three different steps in the PACP and F-PIE results, i.e., from 0 (excellent) to 3 (fair). Please note that there were about 49,000 pipes for which there were no inspection records 
(represented by No-PACP data), so the higher percentage of excellent may not reflect the actual condition of the pipes in the field.

\section{- Results for Selected Pipe Sections}

The Performance Index was calculated for selected pipe sections (manhole to manhole). The sample pipe data provided by City B were used to obtain the performance index. However, some required data were not available. When this was the case, the parameters were derived from similar pipe data, and there was some possibility that the data approximated the actual field status.

\section{Sample 1: Pipe ID 38633}

The pipe ID 38633 was selected for sample calculations. Detailed information about the pipe is given in Table 5.1.

Table 5.1 Sample 1 Pipe Data

\begin{tabular}{|l|l|l|l|}
\hline No. & Parameter & Unit & Value \\
\hline 1 & Pipe Age & Year & 60 \\
\hline 2 & Pipe Depth & Feet & 13.05 \\
\hline 3 & Pipe Diameter & Inch & 8 \\
\hline 4 & Pipe Length & Feet & 39.24 \\
\hline 5 & Pipe Slope & Percent & 10.45 \\
\hline 6 & Density of Connection & Number/100ft & 5.10 \\
\hline 7 & Flooding & Type & No \\
\hline 8 & Ground Cover & Type & Asphalt \\
\hline 9 & Location (Traffic) & Feet & 0.00 (located \\
\hline 10 & Proximity to trees & Feet & 401.69 \\
\hline 11 & Soil Disturbance & Type & No \\
\hline 12 & Soil Resistivity & mV & 0 (very corrosive) \\
\hline 13 & Soil Type & Type & 0 Gravel \\
\hline
\end{tabular}

* Parameters indicated in red are unfavorable parameters 


\section{PACP Rating Index:}

The value of the pipe rating by PACP is "0 (No PACP data)". Inspection data were not available for this pipe section.

F-PIE:

The Performance Index of this pipe has a value of "2" (good). This implies that the pipe has a low likelihood of failure. The highlighted parameters are unfavorable to the performance index.

W-PIE:

The Performance Index of this pipe has a value of "1" (very good). This implies that the pipe is not in danger of failure. The highlighted parameters are unfavorable to the performance index.

\section{Index Comparison: No data, Good, and Very Good}

The PACP rating index of pipe ID 38633 was not available since the pipe had not been inspected. The performance index by F-PIE value "2" indicates good pipe performance. The performance index by W-PIE value "1" indicates very good pipe performance. The pipe performance is not excellent due to different factors, such as old pipe age, corrosive soil conditions, and the proximity to major highways. These parameters affect wastewater pipes and may lead to failure due to corrosion and structural failure. 
Sample 2: Pipe ID 38720

Pipe ID 38720 was selected for sample calculations. Details of this pipe are given in Table 5.2.

Table 5.2 Sample 2 Pipe Data

\begin{tabular}{|l|l|l|l|}
\hline No. & Parameter & Unit & Value \\
\hline 1 & Pipe Age & Year & 75 \\
\hline 2 & Pipe Depth & Feet & 14.9 \\
\hline 3 & Pipe Diameter & Inch & 24 \\
\hline 4 & Pipe Length & Feet & 727.01 \\
\hline 5 & Pipe Slope & Percent & 2.68 \\
\hline 6 & Density of Connection & Number/100ft & 0.14 \\
\hline 7 & Flooding & Type & No \\
\hline 8 & Ground Cover & Type & Asphalt \\
\hline 9 & Location (Traffic) & Feet & $>100$ \\
\hline 10 & Proximity to trees & Feet & $>100$ \\
\hline 11 & Soil Disturbance & Type & No \\
\hline 12 & Soil Resistivity & mV & 10 (very corrosive) \\
\hline 13 & Soil Type & Type & 0 Gravel \\
\hline
\end{tabular}

* Parameters indicated in red are unfavorable parameters

\section{PACP Rating Index:}

The value of the pipe rating by PACP was "4.5." This value is high since the worst value given by the PACP rating index is 5 .

F-PIE:

The Performance Index of this pipe had a value of "5" (Failure). This implies that the pipe is in danger of failure. The parameters indicated in red are unfavorable to the performance index. 
W-PIE:

The Performance Index of this pipe had a value of " 5 " (Failure). This implies that the pipe is in danger of failure. The parameters indicated in red are unfavorable to the performance index. Index Comparison: Poor, Very Poor

The PACP rating index of pipe ID 38720 indicated that the pipe is in a very poor condition, with the value of " 4.5 ". The F-PIE and W-PIE assigns a value of ' 5 ' to this pipe section, consistent with the PACP rating index.

\section{Sample 3: Pipe ID 22733}

Pipe ID 22733 was selected for sample calculations. Details of the pipe are given in Table 5.3 .

Table 5.3 Sample 3 Pipe Data

\begin{tabular}{|l|l|l|l|}
\hline No. & Parameter & Unit & Value \\
\hline 1 & Pipe Age & Year & 57 \\
\hline 2 & Pipe Depth & Feet & 20 \\
\hline 3 & Pipe Diameter & Inch & 8 \\
\hline 4 & Pipe Length & Feet & 336.27 \\
\hline 5 & Pipe Slope & Percent & 0.71 \\
\hline 6 & Density of Connection & Number/100ft & 1.19 \\
\hline 7 & Flooding & Type & No \\
\hline 8 & Ground Cover & Type & Asphalt \\
\hline & & & 10.00 (located \\
9 & Location (Traffic) & Feet & near major road) \\
\hline 10 & Proximity to trees & Feet & $>100$ \\
\hline 11 & Soil Disturbance & Type & No \\
\hline 12 & Soil Resistivity & mV & 170000 \\
\hline 13 & Soil Type & Type & 0 Gravel \\
\hline
\end{tabular}

* Parameters indicated in red are unfavorable parameters 


\section{PACP Rating Index:}

The value of the pipe rating by PACP was " 2.9 ". This value is considered average.

\section{F-PIE:}

The Performance Index of this pipe had a value of " 4 " (poor). This implies that the pipe is in danger of failure. The parameters indicated in red are unfavorable to the performance index.

\section{W-PIE:}

The Performance Index of this pipe had a value of " 4 " (poor). This implies that the pipe is in danger of failure. The parameters indicated in red are unfavorable in the performance index.

\section{Index Comparison: Average, Poor, and Poor}

The PACP rating index of pipe ID 22733 indicated that the pipe is in average condition with the value of "2.9". The performance index value of ' 4 ' indicated poor pipe performance due to for example, a unusually low slope, pipe age, and proximity to a major highway.

\section{Case Study: City $\boldsymbol{H}$}

The City H provided 14 shape-files, 29 geo-database tables and around 1200 Hansen file tables. The shape-files consists mainly of the main pipelines, laterals, and sewer facilities which consist of manholes, rehabilitation work related files, transportation, soils, catch basins and city limits data. The sewer main shape file consists of all the sewer mains within the City $\mathrm{H}$ city limits. The sewer main attributes table consists of various pipe parameters such as Pipe ID, pipe street address, pipe shape, diameter, material, length, and elevation of the upstream and downstream nodes. Also, the slope of the pipe was calculated and added as an attribute. The surface cover and geographical location of each pipe are given along with the sewer shed and basin references. Although a few columns were left blank, information was collected for around 40,000 pipes. More detail about the data supplied by City $\mathrm{H}$ can be found in appendix E. The data provided by City $\mathrm{H}$ includes 12 selected parameters that used in this pilot study. 


\section{Parameters}

- Pipe age

- Pipe diameter

- Pipe depth

- Pipe slope

- Pipe length

- Pipe material

- Traffic

- Soil type

- Flooding

- Groundcover

- Number of connections

- Pipe condition (In-house)

The parameters listed above were stored as attributes in mainline GIS shape-file. However, age was known for approximately 900 pipes. Traffic data was derived from the arterial shape-file and a spatial analysis tool was used to approximate the distances from pavements to pipes. Soil data was provided in separate shape-files. The number of connections was calculated from counting lateral pipes in lateral shape-file that intersect with a pipe of interest. Sewer inspections were used to identify defects and sources of I\&I including, CCTV, smoke test, dye test, flow monitoring, rain monitoring, building service connection location/inspection, and flow isolation data.

Defects were rated based on City H's in-house defects rating. The in-house system gave a rating of 1-5 for each pipe section. Based on the data received, both weighted factor and fuzzy logic models were run and the results are presented. Figure 5.8 presents the in-house rating index for City $\mathrm{H}$. The pipes were rated on a scale of 1-5 where 1 (green) represents the pipe in excellent condition and 5 (red) in failed condition. The pipes that do not have inspection data (0) which indicated in black were assumed excellent for the purpose of calculation. 


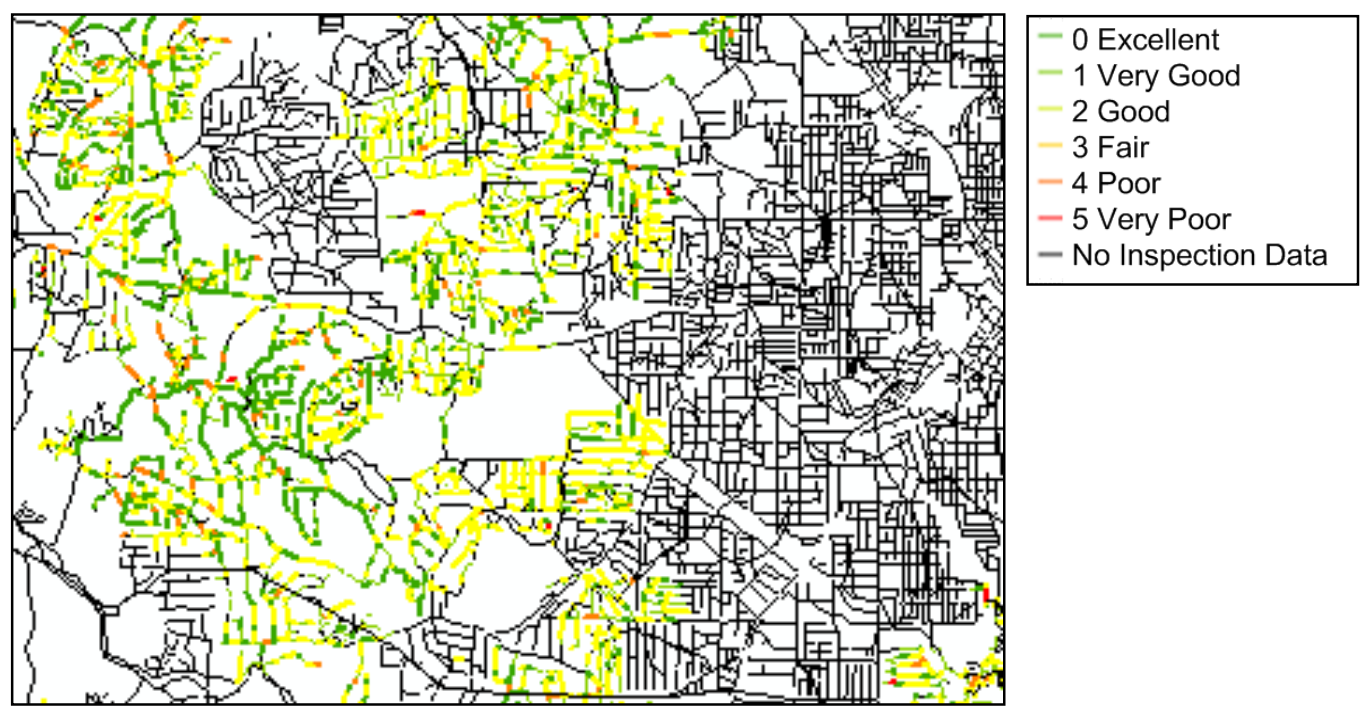

Figure 5.8 In-House Rating Index

\section{- W-PIE and F-PIE Results}

Figures 5.9 and 5.10 show the results of the performance index calculated by W-PIE and F-PIE, where 0 represents the pipe in excellent condition and 5 represents a failed condition. The result is mapped to GIS to illustrate the distribution of the performance index. Figure 5.11 shows a bar chart of the performance index calculated using In-house data, W-PIE and F-PIE to illustrate the distribution of the results, which the $x$-axis is the performance index and $y$-axis is the number of pipes that fall within each value. As shown in Figure 5.9 and 5.10, the majority of the performance index of City $\mathrm{H}$ is 0 and 1, which indicate excellent and good, respectively. From the results presented here, the failed and poor pipes are aggregated along 3 areas of the city (north, west, and south). Further investigation is needed in order to determine the causes of failures. It should be noted that, even though the in-house rating index was not available for a pipe, which may imply that the pipe had not been inspected, both F-PIE and W-PIE were able to calculated scores for the pipes. 


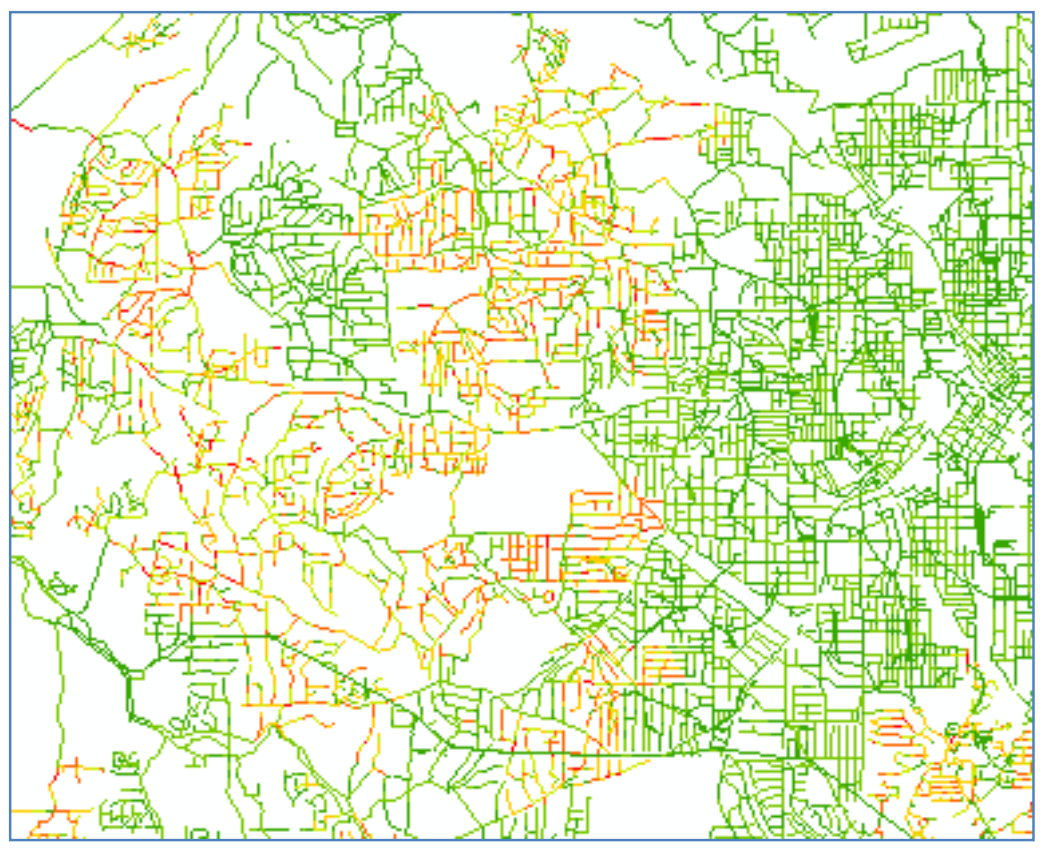

\begin{tabular}{|l}
\hline-0 Excellent \\
-1 Very Good \\
-2 Good \\
-3 Fair \\
-4 Poor \\
-5 Very Poor \\
- No Inspection Data
\end{tabular}

Figure 5.9 Performance Index by W-PIE

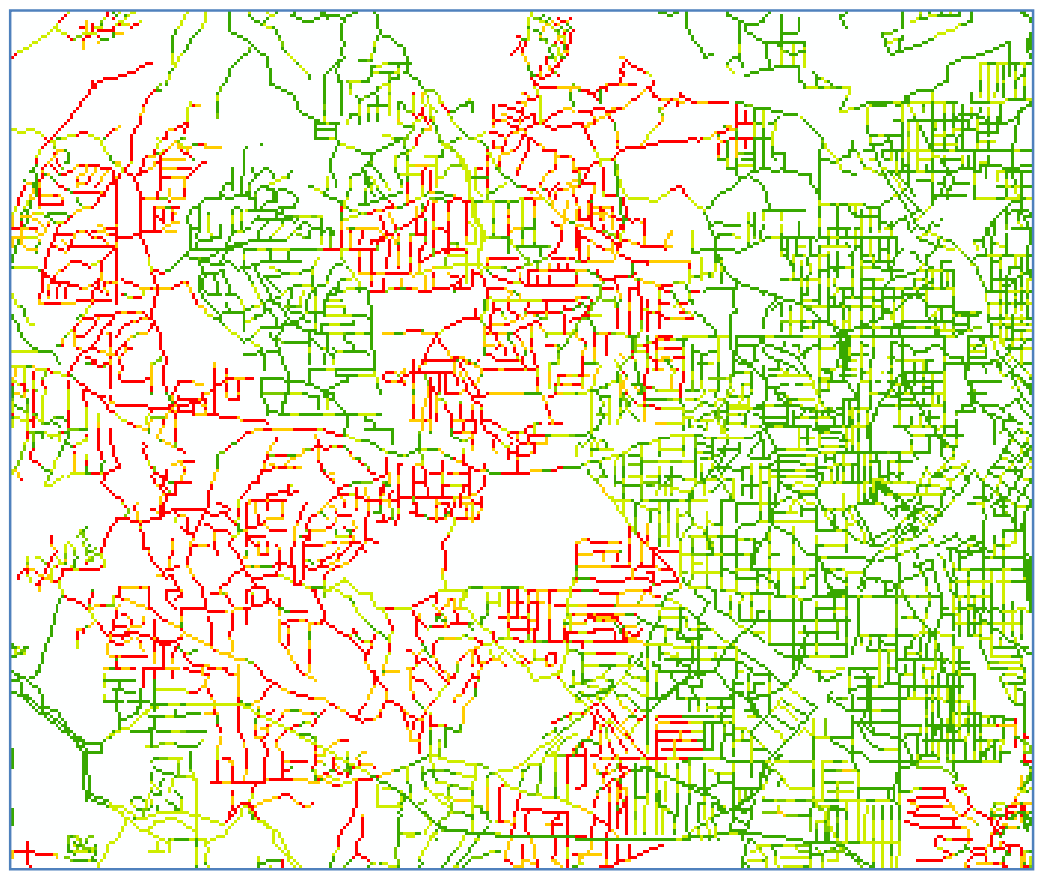

-0 Excellent
-1 Very Good
-2 Good
-3 Fair
-4 Poor
-5 Very Poor
- No Inspection Data

Figure 5.10 Performance Index by F-PIE 


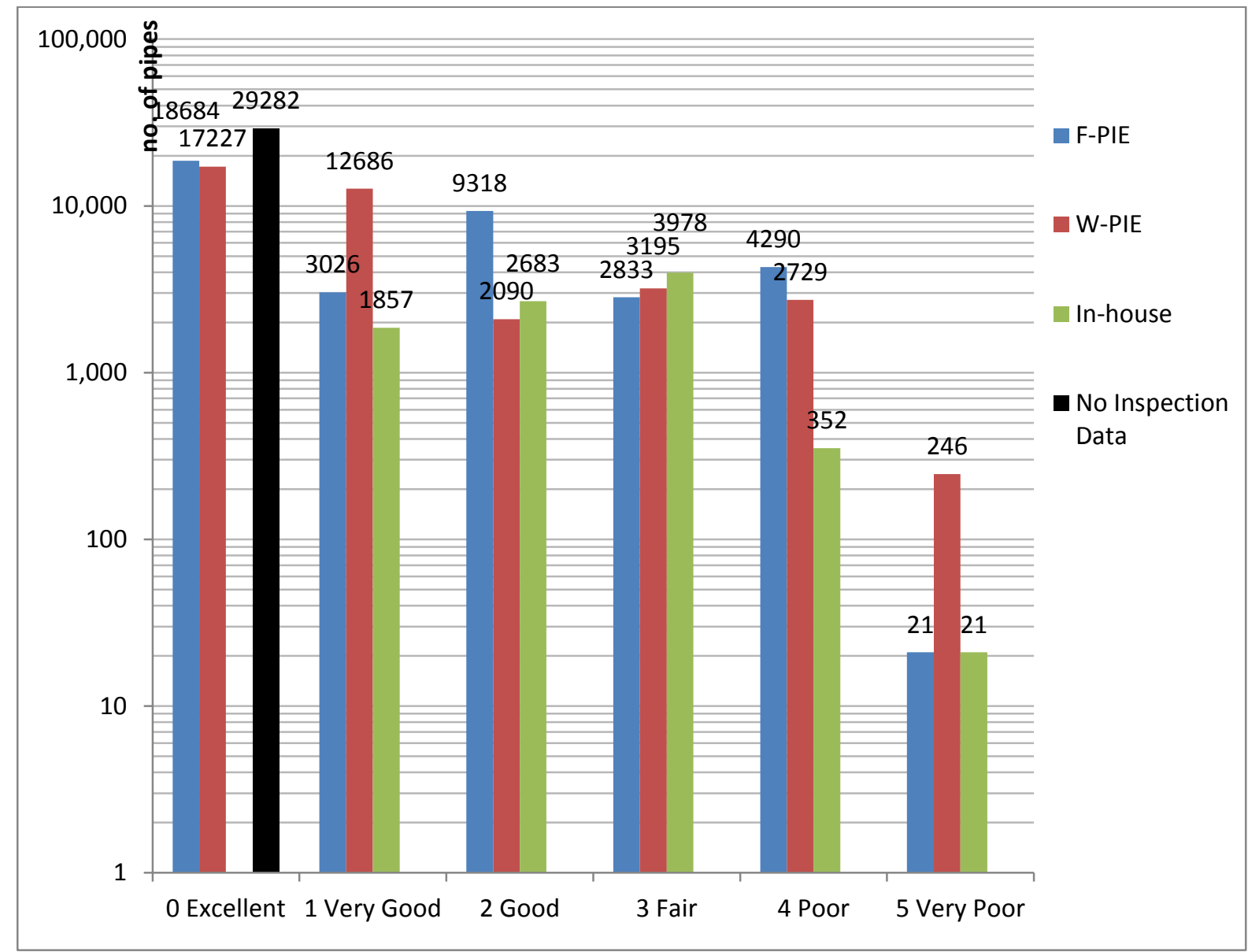

Figure 5.11 Performance Index by In-House Rating System, W-PIE and F-PIE

\section{- Results for Selected Pipe Sections}

The performance index was calculated for selected pipe sections (manhole to manhole). The sample pipe data provided by City $\mathrm{H}$ was used to obtain the performance index. However, some required data were not available, in this case, the parameters were derived from similar data and there is a possibility that the data do not resemble the actual field status.

\section{Sample 1: Pipe ID 13830102801T13830102401}

The pipe ID 13830102801T13830102401was selected for sample calculations. Detailed information of the pipe is given in Table 5.4. 
Table 5.4 Sample 1 Pipe Data

\begin{tabular}{|l|l|l|l|}
\hline No. & Parameter & Unit & Value \\
\hline 1 & Pipe Age & Year & No data \\
\hline 2 & Pipe Depth & Feet & 9 \\
\hline 3 & Pipe Diameter & Inch & 10 \\
\hline 4 & Pipe Length & Feet & 99 \\
\hline 5 & Pipe Slope & Percent & 0.44 \\
\hline 6 & Density of Connection & Number & 1 \\
\hline 7 & Ground Cover & Type & Asphalt, Concrete \\
\hline & & & 0.00 (located \\
8 & Location (Traffic) & Feet & under major road) \\
\hline 9 & Soil Type & Type & Clay \\
\hline
\end{tabular}

* Parameters indicated in red are unfavorable parameters

\section{In-house Rating Index:}

The value of the pipe rating by In-house was " 0 (No data)". Inspection data was not available for this pipe section.

W-PIE:

The Performance Index of this pipe had a value of "1 (very good)"and it was not in danger of failure. The parameters highlighted were unfavorable in the performance index.

F-PIE:

The performance index of this pipe had a value of " 2 (good)" and the pipe was not in danger of failure. The parameters highlighted were unfavorable to the performance index.

\section{Index Comparisons: No data \& Average}

The in-house rating index of this pipe ID 13830102801T13830102401 was not available since the pipe had not been inspected. The W-PIE gave value ' 1 ' and F-PIE gives value ' 2 ' to this 
pipe section due to factors such as shallow depth and a location close to major highways, a lower than average slope, and a clayey soils. These parameters affect wastewater pipes and may lead to failure due to excessive stress and blockage.

\section{Sample 2: Pipe ID 13840202101T13840202001}

The pipe ID 13840202101T13840202001 was selected for sample calculations. Details of this pipe are given in Table 5.5.

Table 5.5 Sample 2 Pipe Data

\begin{tabular}{|l|l|l|l|}
\hline No. & Parameter & Unit & Value \\
\hline 1 & Pipe Age & Year & No data \\
\hline 2 & Pipe Depth & Feet & 10 \\
\hline 3 & Pipe Diameter & Inch & 8 \\
\hline 4 & Pipe Length & Feet & 300 \\
\hline 5 & Pipe Slope & Percent & 10 \\
\hline 6 & Density of Connection & Number/100ft & 1 \\
\hline 7 & Ground Cover & Type & Asphalt, Concrete \\
\hline 8 & Location (Traffic) & Feet & 1 \\
\hline 9 & Soil Type & Type & Clay \\
\hline
\end{tabular}

* Parameters indicated in red are unfavorable parameters

\section{In-house Rating Index:}

The value of the pipe rating by PACP was " 4 ". This value is poor, since the worst value given in the PACP rating index is 5 .

W-PIE:

The Performance Index of this pipe had a value of "5 (poor)". This implies that the pipe was in danger of failure. The parameters indicated in red were unfavorable in the performance index. 
F-PIE:

The Performance Index of this pipe had a value of "4 (poor)". This implies that the pipe was in danger of failure. The parameters indicated in red are unfavorable in the performance index.

Index Comparisons: No data \& Average

The in-house rating of this pipe indicated that the pipe was in very poor condition with a value of "4". The W-PIE gives a value of '5' and F-PIE gave a value of '4' to this pipe section consistent with the PACP rating index. The parameters affecting this pipe were pipe diameter, pipe length, pipe slope, and soil type. Very steep slope creates very high flow velocity and may lead to surface wear.

\section{Case Study: Washington Suburban Sanitary Commission (WSSC)}

The WSSC, one of the leaders in the wastewater pipe infrastructure, has a special team devoted to the analysis its current system. WSSC staffs and their consultants have developed their own performance rating model. The WSSC's performance rating consists of level 1 and level 2 evaluations which level 2 rating is for pipes that have been inspected and level 1 is for the otherwise. The WSSC's model is comprised of an age, structural, operational and maintenance (O\&M) based scores which includes parameters such as material type, diameter, length, construction year, depth, for example. This WSSC model was used to compare the results of the developed fuzzy inference performance model based on similar inputs.

We analyzed the data received from WSSC and evaluated each pipe section (ManholeManhole) using fuzzy inference performance model. The provided data was for a specific area called "Broad Creek basin". The reinforced concrete pipes were selected for this study. Altogether, there were approximately 8000 pipes where, about 100 pipes were inspected. The data included level 1 and level 2 data tables. In total, 8 parameters were used in this pilot study.

\section{Parameters}

- Pipe age 
- Pipe diameter

- Pipe depth

- Pipe length

- Pipe material

- Number of connections

$-\mathrm{H} 2 \mathrm{~S}$

- Pipe condition (PACP defect rating )

The performance index was calculated using the fuzzy inference model and compared to level 1 and level 2 scores given by WSSC. The pipes were rated based on the scale (1-5) where 1 represents the pipe in excellent condition, and 5 represents failed condition.

Figure 5.12 presents WSSC's L2 score versus performance index (F-PIE). It shows a strong correlation between overall score and fuzzy inference performance index. A scatter plot of the results shows a clear linear trend. Figure 5.13 presents WSSC's overall score vs. fuzzy inference calculated without PACP inspection data. Fuzzy inference model gives the score 1 to 3 resembling WSSC's L1 score, however, we can see a slight variation between them.

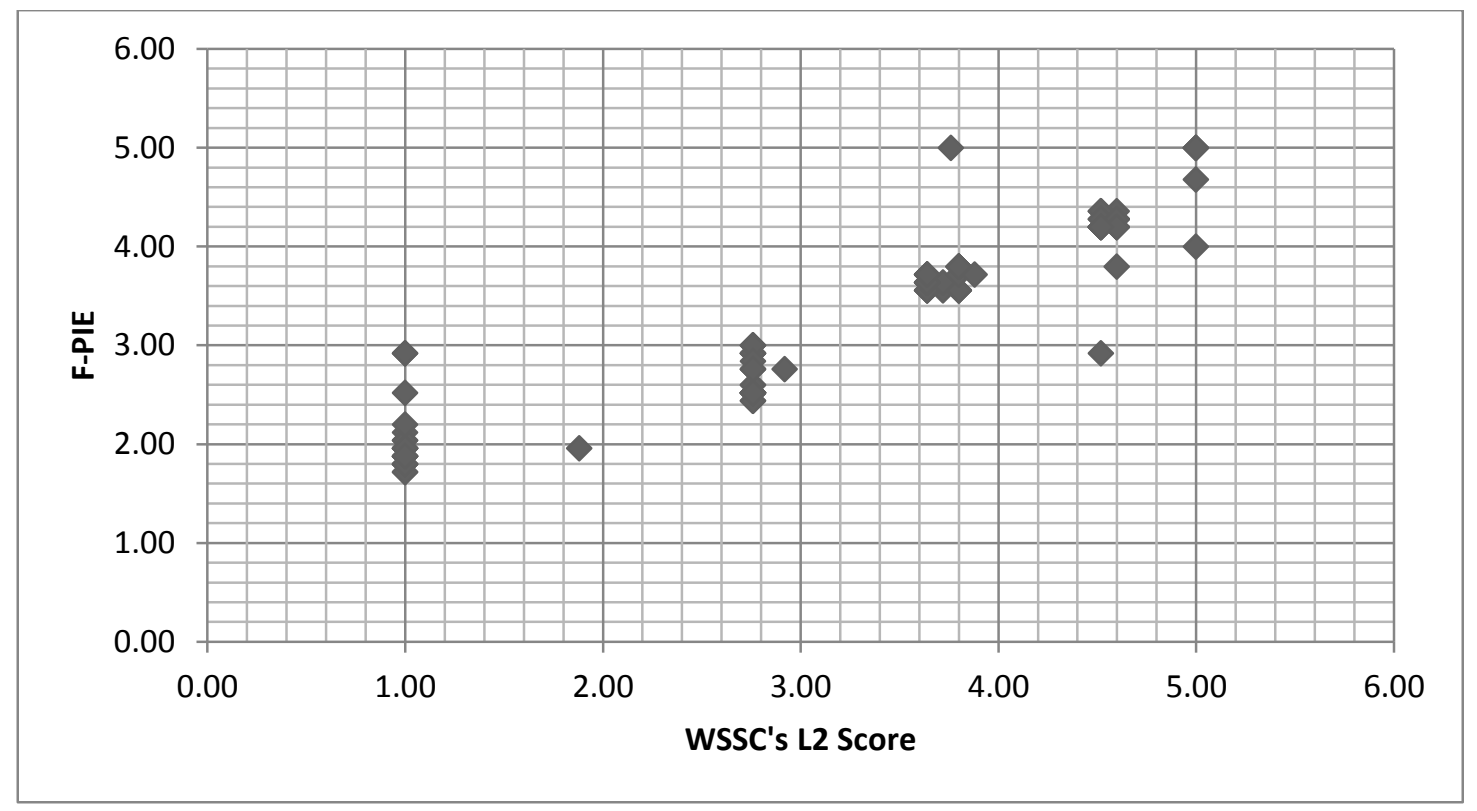

Figure 5.12 WSSC's L2 Score vs. Fuzzy Inference Performance Index 


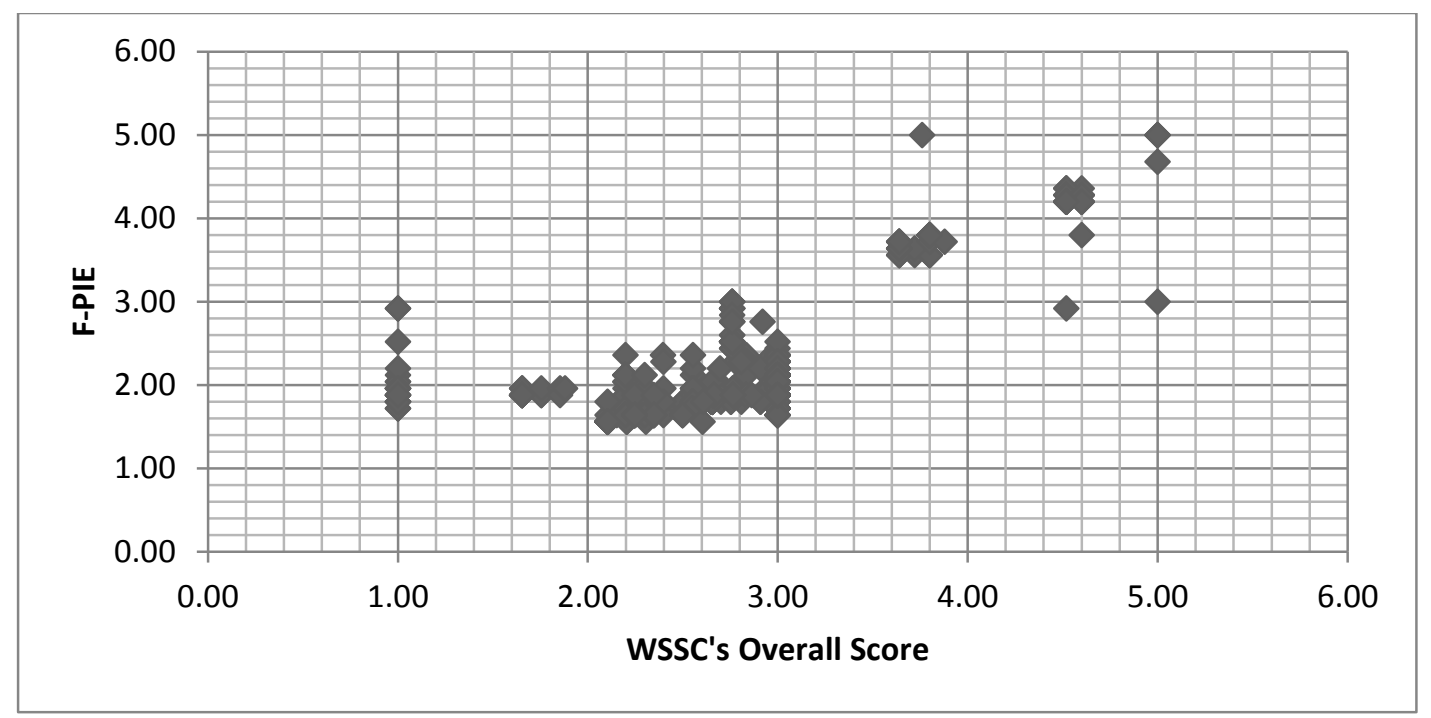

Figure 5.13 WSSC's Overall Condition Score vs. Fuzzy Inference Performance Index

We did try to compare the L1 scores with the fuzzy inference model results omitted the inspection data, however the correlation between them was not clear as shown in Figure 5.14. Perhaps, more parameters affecting the pipe performance should be considered in this exercise to reflect actual conditions of the pipes. All the results calculated by the fuzzy inference model can be found in excel file attached.

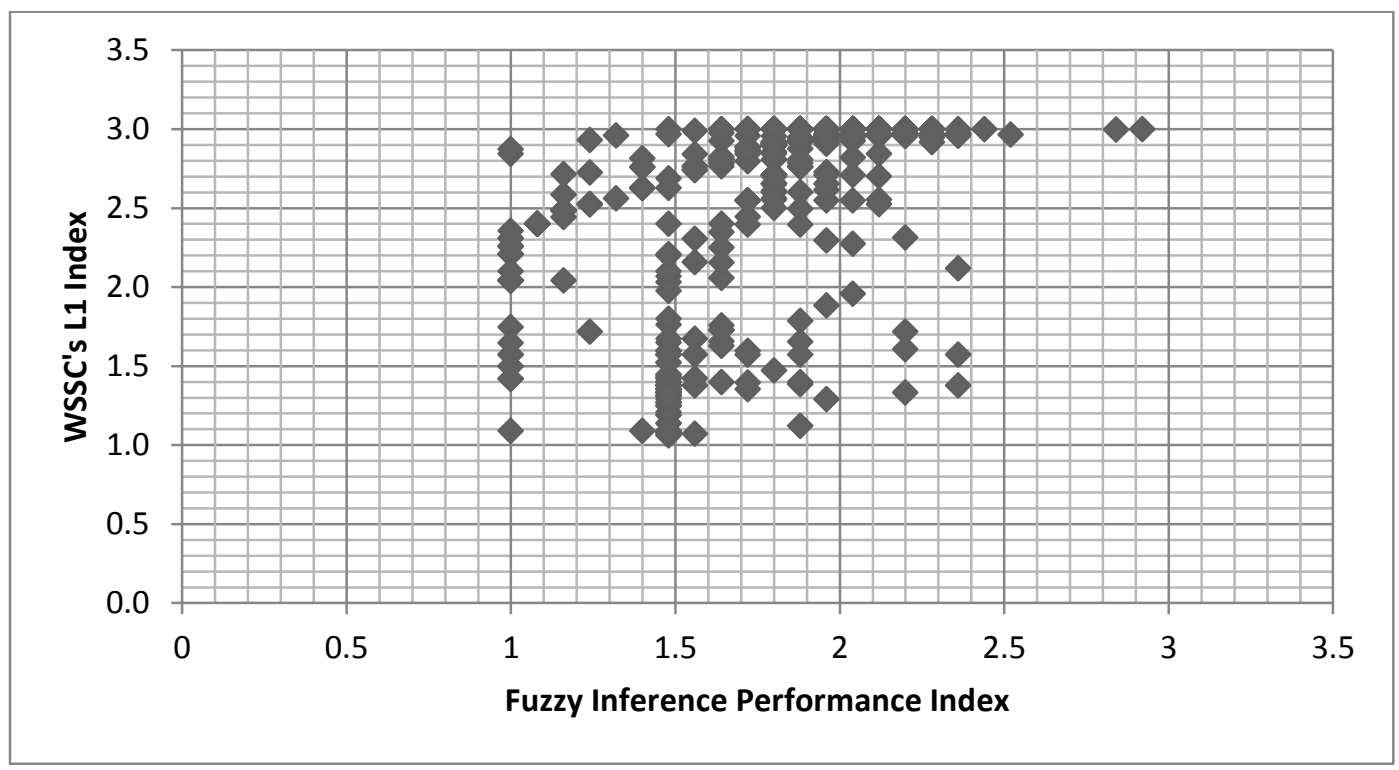

Figure 5.14 WSSC's L1 Score vs. Fuzzy Inference Performance Index (without inspection data) 


\subsection{Summary}

The performance model is a robust performance rating system created to evaluate wastewater pipes that uses various parameters that affect wastewater pipes as inputs. It can help utilities in planning, prioritization, maintenance, and repair/rehabilitation/replacement. The performance model that was developed was evaluated through a pilot study utilizing utility data across the nation to test the methodology. Due to financial and time constraints it is almost impossible to physically dig up or perform lab testing on numerous sample pipes to determine their condition so CCTV inspection and other models were used to evaluate the pipe performance based on the existing utility database. Based on the evaluation, it can be concluded that the model performs well due to the input values from the test and real data processes producing expected outputs and consistent with the expert opinions.

The results presented were from the models built for evaluating pipes for the utilities targeted for pilot studies and as anticipated, some of the parameters, ranges, and weights are not applicable to all utilities. However, a generic model provides sufficient knowledge and serves as a guideline for establishing a performance evaluation program. In addition, a generic model is essential to compare results to other utilities. The results presented in this study serve as a filter for further and more granular localized rehabilitation and replacement methodologies. The correct intervention decision should be based on a combination of specific, localized defects found within the pipe and the economic consequences.

The models provide a noticeable improvement from the current practice of using inspection data (CCTV, dye test, or other inspection data) as a mean to evaluate the wastewater pipe infrastructure system alone, because the model accounts for inspection data and other parameters that influence pipe performance. The fuzzy logic was good for approximate reasoning and incomplete information. We found the fuzzy inference model has many advantages over conventional weighted-factor model. The fuzzy inference model provided more sensitive results, whereas the weighted-factor model provided more static results since the weights assigned to each parameter were fixed and distributed throughout all parameters in the model. In addition, the fuzzy inference model accounted for the combination effect of dependent parameters. 


\section{CHAPTER 6}

\section{DeVelopment of A Framework for Wastewater PiPe Performance Prediction}

Accurate prediction of wastewater pipe structural and functional deterioration plays an essential role in asset management and capital improvement planning. Deterioration/Prediction modeling is a crucial step in assessing the remaining asset life. Sound infrastructure deterioration models are essential for accurately predicting future conditions that, in turn, are key inputs to effective maintenance, repair, and rehabilitation decision making. Utilities rely significantly on CCTV (Closed-circuit television) inspection in evaluating pipes and making rehabilitation decisions. However, the CCTV doesn't capture all data needed in predicting wastewater pipe failures. In the chapter 5, we investigated the methods to evaluate the pipe condition and performance through other important parameters including structural, environmental, operational, and other important parameters. The performance index has been developed.

Different methods had been explored in developing a performance prediction Model as stated in chapter 2. Unlike the other infrastructure systems, such as pavement or bridge, wastewater pipe system data are sacred, and time dependent data are not available for most utilities. Due to unavailability of time dependent data, the choice is limited. The application of Markov 
prediction has been widely considered for different infrastructure assets such as pavements (Butt 1987, Carnahan 1987), bridges (Jiang 1988) and storm water pipe systems (Micevski, 2002). Particularly, deterioration prediction models were developed for wastewater pipe system using the Markov prediction approach with data from sewer pipe inspection as an input data (Abraham, 1998; Kathula, 2001; Wirahadikusumah, 2001; Baik, 2006; Sinha 2007; Park 2009).

A reliable assessment of the current condition is very important for dependable application of the Markov chain in forecasting deterioration. Because, if the base information is incorrect, then the forecasting model based on that information will be unreliable (Madanat, 1995). In this chapter, a Markov performance prediction model are presented utilizing performance index score generated from wastewater pipe performance index presented in chapter 4 .

\subsection{Markov Chains}

A Markov chain is a random process and it's usually defined for a discrete-time set. A random process has the Markov property if the conditional probability of future event depends on the current state but not any past state. This memory-less property can be expressed for the random variable $\left(X_{t}\right)$ as;

$$
P\left(X_{t+1}=i_{t+1} \mid X_{t}=i_{t}, X_{t-1}=i_{t-1}, \ldots, X_{1}=i_{1}, X_{0}=i_{0}\right)=P\left(X_{t+1}=i_{t+1} \mid X_{t}=i_{t}\right)
$$

Where,

$i_{t}=$ the state of the process at time $t$

$P=$ the conditional probability of the future event

The fixed probability that the system changes from state $\mathrm{i}$ to state $\mathrm{j}$ can is called a transition probability and can be written as

$$
P\left(X_{t+1}=j \mid X_{t}=i\right)=p_{i j}
$$

The transition matrix $(\mathrm{P})$ represents transition probability where the order of the matrix $(\mathrm{m})$ is equal to the number of outcome states. The transition matrix $\mathrm{P}$ is shown in equation 6-3. 


$$
. P=\left[\begin{array}{cccc}
p_{11} & p_{12} & . . & p_{1 m} \\
p_{21} & p_{22} & . . & p_{2 m} \\
: & : & : & : \\
p_{m 1} & p_{m 2} & . . & p_{m m}
\end{array}\right]
$$

Where,

$m=$ the number of performance states

$p_{i j}=$ the transition probability from state $i$ to state $j$

$P=$ the transition probability matrix

\subsection{Expected Value Method}

The Expected Value Method has been extensively used to estimate the transition probability for various infrastructure assets because of its simplicity and ease of uses (Park, 2009). In this method, the data are grouped into categories where each categories consists of the same factors for example, same material, and/or diameter. Subsequently, the regression analysis is performed between rating and time. A non-linear optimization technique is utilized to estimates the transition probabilities as shown in equation 6-4.

$$
\text { Minimize } \sum_{t=1}^{N}|Y(t)-E(t, P)|
$$

Where,

$\mathrm{t}=$ age of asset

$\mathrm{N}=$ total number of transition period

$\mathrm{Y}(\mathrm{t})=$ Estimated performance from regression function

$\mathrm{E}(\mathrm{t}, \mathrm{P})=$ Expected value of performance at time $\mathrm{t}$ based on Markov chain model

$\mathrm{E}(\mathrm{t}=\mathrm{n}, \mathrm{P})=\left[\begin{array}{lllll}1 & 0 & 0 & 0 & 0\end{array}\right] \mathrm{P}^{(\mathrm{n})} \mathrm{C}^{\mathrm{T}}$

$\left[\begin{array}{lllll}1 & 0 & 0 & 0 & 0\end{array}\right]=$ Initial performance state matrix;

$\mathrm{P}^{(\mathrm{n})}=$ Transition probabilities matrix after $\mathrm{n}$ transition,

$\mathrm{C}^{\mathrm{T}}=$ Transpose of performance rating matrix $\left[\begin{array}{lllll}1 & 2 & 3 & 4 & 5\end{array}\right]^{\mathrm{T}}$ 
The expected performance value, $\mathrm{E}(\mathrm{t}, \mathrm{P})$ can also be expressed as;

$$
E(t, P)=Q^{(n)} S^{T}=Q^{(0)} P^{(n)} S^{T}
$$

Where,

$\mathrm{Q}^{(0)}=$ initial performance vector at stage 0

$\mathrm{Q}^{(\mathrm{n})}=$ performance vector at stage $\mathrm{n}$

$\mathrm{P}^{(\mathrm{n})}=$ probability matrix after $\mathrm{n}$ transitions

$\mathrm{S}^{\mathrm{T}}=$ transpose of the performance rating vector $\mathrm{S}$

\subsection{Performance Prediction Results}

In this research, due to unavailability of time dependent data, the expected value method was selected to estimate the transition probability. The data received from WSSC was utilized to illustrate the implementation of the performance prediction model. Concrete pipes with diameter less than 15" and vitrified clay pipes with diameter less than 15" located in specific area called Broad Creek Basin were selected.

\section{Concrete Pipe Sample}

A zoning concept is applied to reflect nature of the pipe deterioration that the older pipe deteriorates at a more rapidly rate than newer pipes. The five year zone is assumed as results the rate of the performance deterioration does not change and the performance of pipe does not drop more than 1 state in five year period. The estimated performance of the concrete pipes is shown in equation 6-6.

$$
Y(t)=\exp (0.334+0.0128 t)
$$

The matrix $\left[\begin{array}{lllll}1 & 0 & 0 & 0 & 0\end{array}\right]$ is used as the initial performance for the first zone. The optimization equation can be expressed as shown in equation 6-7. 


$$
\begin{aligned}
& \text { Minimize } \quad\left(\left|e^{0.334+0.0128 * 1}-0.3965-\left[\begin{array}{lllll}
1 & 0 & 0 & 0 & 0
\end{array}\right] P_{1}^{(1)}\left[\begin{array}{llll}
1 & 2 & 3 & 4
\end{array}\right]^{T}\right|\right. \\
& +\left|e^{0.334+0.0128 * 2}-0.3965-\left[\begin{array}{lllll}
1 & 0 & 0 & 0 & 0
\end{array}\right] P_{1}^{(2)}\left[\begin{array}{lllll}
1 & 2 & 3 & 4 & 5
\end{array}\right]^{T}\right| \\
& +\left|e^{0.334+0.0128 * 3}-0.3965-\left[\begin{array}{lllll}
1 & 0 & 0 & 0 & 0
\end{array}\right] P_{1}^{(3)}\left[\begin{array}{lllll}
1 & 2 & 3 & 4
\end{array}\right]^{T}\right| \\
& +\left|e^{0.334+0.0128 * 4}-0.3965-\left[\begin{array}{lllll}
1 & 0 & 0 & 0 & 0
\end{array}\right] P_{1}^{(4)}\left[\begin{array}{lllll}
1 & 2 & 3 & 4 & 5
\end{array}\right]^{T}\right| \\
& \left.+\left|e^{0.334+0.0128 * 5}-0.3965-\left[\begin{array}{lllll}
1 & 0 & 0 & 0 & 0
\end{array}\right] P_{1}^{(5)}\left[\begin{array}{lllll}
1 & 2 & 3 & 4 & 5
\end{array}\right]^{T}\right|\right) \quad(6-7)
\end{aligned}
$$

The optimization equation shown in equation 6-8 is used in calculating the transition probability for the second zone,

$$
\begin{aligned}
& \text { Minimize } \quad\left(\left|e^{0.334+0.0128 * 6}-0.3965-\left[\begin{array}{lllll}
1 & 0 & 0 & 0 & 0
\end{array}\right] P_{1}^{(5)} P_{2}^{(1)}\left[\begin{array}{llll}
1 & 2 & 3 & 4
\end{array}\right]^{T}\right|\right. \\
& +\left|e^{0.334+0.0128 * 7}-0.3965-\left[\begin{array}{lllll}
1 & 0 & 0 & 0 & 0
\end{array}\right] P_{1}^{(5)} P_{2}^{(2)}\left[\begin{array}{lllll}
1 & 2 & 3 & 4 & 5
\end{array}\right]^{T}\right| \\
& +\left|e^{0.334+0.0128 * 8}-0.3965-\left[\begin{array}{lllll}
1 & 0 & 0 & 0 & 0
\end{array}\right] P_{1}^{(5)} P_{2}^{(3)}\left[\begin{array}{lllll}
1 & 2 & 3 & 4 & 5
\end{array}\right]^{T}\right| \\
& +\left|e^{0.334+0.0128 * 9}-0.3965-\left[\begin{array}{lllll}
1 & 0 & 0 & 0 & 0
\end{array}\right] P_{1}^{(5)} P_{2}^{(4)}\left[\begin{array}{lllll}
1 & 2 & 3 & 4 & 5
\end{array}\right]^{T}\right| \\
& \left.+\left|e^{0.334+0.0128 * 10}-0.3965-\left[\begin{array}{lllll}
1 & 0 & 0 & 0 & 0
\end{array}\right] P_{1}^{(5)} P_{2}^{(5)}\left[\begin{array}{lllll}
1 & 2 & 3 & 4 & 5
\end{array}\right]^{T}\right|\right) \quad(6-8)
\end{aligned}
$$

By utilizing the similar method, the transition probabilities for every zone are calculated. The transition probability for this selected category is presented in Table 6.1. 
Table 6.1 Transition Probability

\begin{tabular}{|l|r|l|r|l|r|r|}
\hline Period & Zone & P11 & P22 & P33 & P44 & P55 \\
\hline $0-5$ & 1 & 0.981997 & 0.968847 & 0.967114 & 0.999241 & 1 \\
\hline $5-10$ & 2 & 0.981655 & 0.968658 & 0.993497 & 0.999998 & 1 \\
\hline $10-15$ & 3 & 0.985578 & 0.944035 & 0.977581 & 0.998876 & 1 \\
\hline $15-20$ & 4 & 0.982905 & 0.963625 & 0.955985 & 0.971586 & 1 \\
\hline $20-25$ & 5 & 0.985801 & 0.960333 & 0.938223 & 0.957547 & 1 \\
\hline $25-30$ & 6 & 0.985367 & 0.952426 & 0.950492 & 0.968866 & 1 \\
\hline $30-35$ & 7 & 0.981388 & 0.958702 & 0.957233 & 0.963429 & 1 \\
\hline $35-40$ & 8 & 0.979818 & 0.959858 & 0.952832 & 0.96314 & 1 \\
\hline $40-45$ & 9 & 0.979679 & 0.956258 & 0.950029 & 0.961512 & 1 \\
\hline $45-50$ & 10 & 0.977773 & 0.951654 & 0.949613 & 0.962839 & 1 \\
\hline $50-55$ & 11 & 0.974557 & 0.946309 & 0.948832 & 0.965717 & 1 \\
\hline $55-60$ & 12 & 0.969214 & 0.94457 & 0.947396 & 0.964156 & 1 \\
\hline $60-65$ & 13 & 0.965263 & 0.939083 & 0.94353 & 0.962314 & 1 \\
\hline $65-70$ & 14 & 0.958284 & 0.931866 & 0.93946 & 0.961385 & 1 \\
\hline $70-75$ & 15 & 0.955494 & 0.922076 & 0.929256 & 0.957541 & 1 \\
\hline $75-80$ & 16 & 0.939681 & 0.913643 & 0.924229 & 0.950466 & 1 \\
\hline $80-85$ & 17 & 0.921053 & 0.892477 & 0.907434 & 0.94987 & 1 \\
\hline $85-90$ & 18 & 0.903148 & 0.877668 & 0.891412 & 0.92918 & 1 \\
\hline $90-95$ & 19 & 0.885192 & 0.855385 & 0.853848 & 0.898943 & 1 \\
\hline $95-100$ & 20 & 0.781588 & 0.75149 & 0.779944 & 0.85812 & 1 \\
\hline $100-105$ & 21 & 0.529037 & 0.52293 & 0.586665 & 0.721003 & 1 \\
\hline $105-110$ & 22 & 0 & 0 & 0 & 0 & 1 \\
\hline
\end{tabular}

The expected performance at given time can be calculated by;

$$
E(t, P)=Q^{(n)} S^{T}=Q^{(0)} P^{(n)} S^{T} \quad(6-9)
$$


For example, assuming that there will be no repair or rehabilitation done to the pipeline. 30 year expected performance would equal to;

$$
\begin{aligned}
\mathrm{E}(30, \mathrm{P}) & =\left[\begin{array}{lllll}
1 & 0 & 0 & 0 & 0
\end{array}\right] P_{1}^{(5)} P_{2}^{(5)} P_{3}^{(5)} P_{4}^{(5)} P_{5}^{(5)} P_{6}^{(5)}\left[\begin{array}{llll}
1 & 2 & 3 & 4
\end{array}\right]^{T} \quad(6-10) \\
& =1.6538
\end{aligned}
$$

After the performance of every zone are obtained, the performance value can be plotted against time as shown in Figure 6.1.

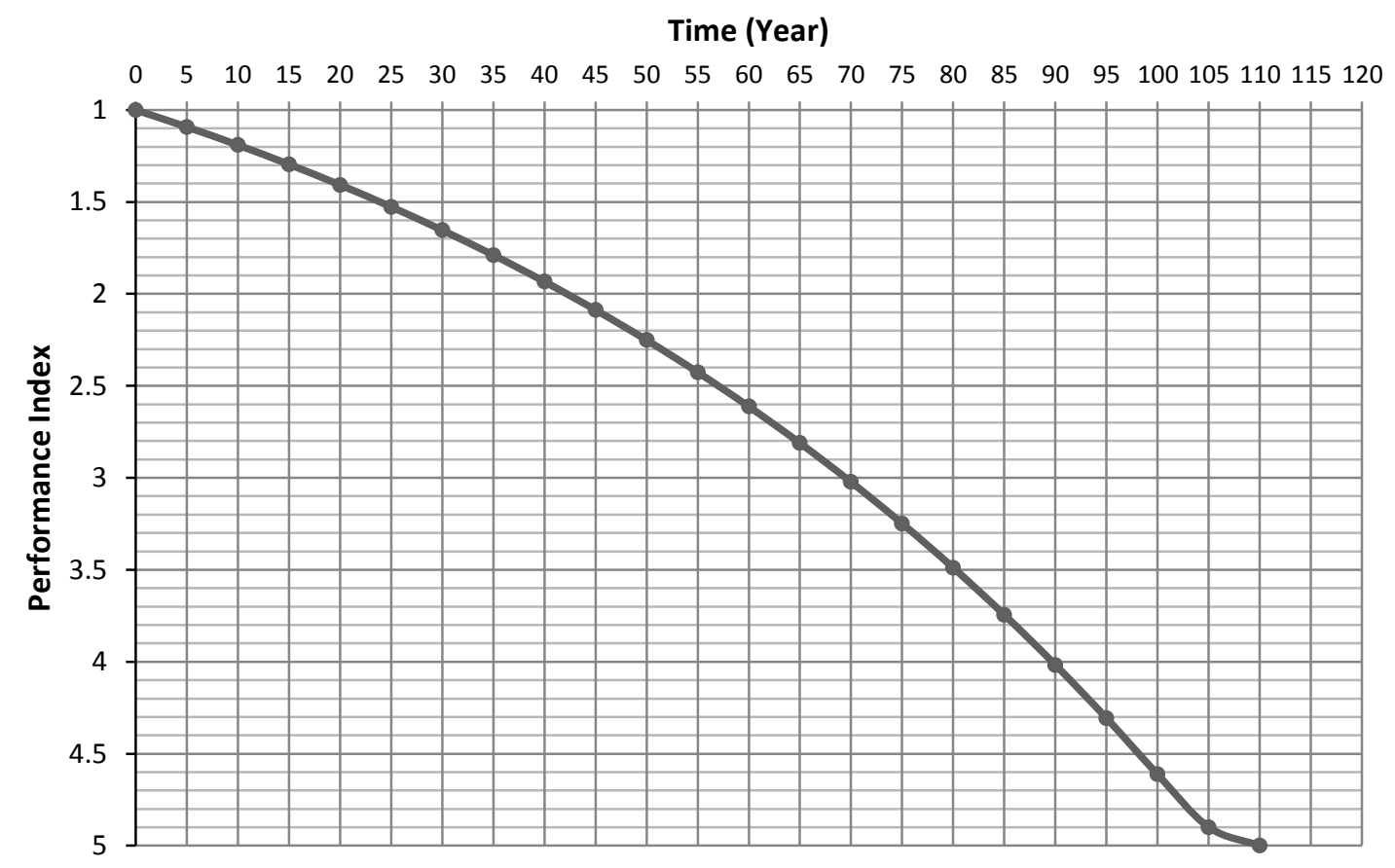

Figure 6.1 Performance Prediction for Concrete Pipe

\section{Vitrified Clay Pipe Sample}

In order to compare the prediction curves the second sample was tested. By following the same procedure for concrete pipe, the estimated performance of the vitrified clay pipe is shown in equation 6-11.

$$
Y(t)=\exp (0.0845+0.0129 t)
$$


The transition probabilities for every zone are calculated. The transition probability for the vitrified clay pipe is presented in Table 6.2.

Table 6.2 Transition Probability for Vitrified Clay Pipe

\begin{tabular}{|c|c|c|c|c|c|c|}
\hline Period & Zone & P11 & $\mathbf{P 2 2}$ & P33 & P44 & P55 \\
\hline $0-5$ & 1 & 0.985798 & 0.973092 & 0.997184 & 0.999048 & 1 \\
\hline $5-10$ & 2 & 0.986272 & 0.966708 & 0.978219 & 0.999579 & 1 \\
\hline $10-15$ & 3 & 0.98836 & 0.951055 & 0.977319 & 0.981967 & 1 \\
\hline $15-20$ & 4 & 0.987125 & 0.963867 & 0.961093 & 0.976458 & 1 \\
\hline $20-25$ & 5 & 0.990621 & 0.959068 & 0.933053 & 0.957062 & 1 \\
\hline $25-30$ & 6 & 0.984379 & 0.960669 & 0.977624 & 0.989274 & 1 \\
\hline $30-35$ & 7 & 0.990174 & 0.957111 & 0.947576 & 0.958783 & 1 \\
\hline $35-40$ & 8 & 0.987385 & 0.962985 & 0.949585 & 0.956768 & 1 \\
\hline $40-45$ & 9 & 0.991156 & 0.956235 & 0.940546 & 0.941877 & 1 \\
\hline $45-50$ & 10 & 0.980499 & 0.954613 & 0.962963 & 0.971709 & 1 \\
\hline $50-55$ & 11 & 0.980233 & 0.953846 & 0.957039 & 0.97027 & 1 \\
\hline $55-60$ & 12 & 0.975705 & 0.945001 & 0.963217 & 0.980764 & 1 \\
\hline $60-65$ & 13 & 0.97459 & 0.950445 & 0.957749 & 0.970131 & 1 \\
\hline $65-70$ & 14 & 0.972235 & 0.946756 & 0.954845 & 0.96652 & 1 \\
\hline $70-75$ & 15 & 0.967194 & 0.942699 & 0.952539 & 0.966445 & 1 \\
\hline $75-80$ & 16 & 0.960944 & 0.939874 & 0.944985 & 0.967596 & 1 \\
\hline $80-85$ & 17 & 0.957012 & 0.93253 & 0.939691 & 0.962436 & 1 \\
\hline $85-90$ & 18 & 0.948775 & 0.925532 & 0.933206 & 0.956235 & 1 \\
\hline $90-95$ & 19 & 0.937845 & 0.914714 & 0.923004 & 0.949589 & 1 \\
\hline $95-100$ & 20 & 0.917433 & 0.88845 & 0.909044 & 0.946641 & 1 \\
\hline $100-105$ & 21 & 0.897061 & 0.876849 & 0.885889 & 0.926221 & 1 \\
\hline $105-110$ & 22 & 0.853885 & 0.829356 & 0.847973 & 0.900395 & 1 \\
\hline $110=115$ & 23 & 0.745871 & 0.725907 & 0.763004 & 0.842223 & 1 \\
\hline $115-120$ & 24 & 0.375409 & 0.385279 & 0.482992 & 0.637623 & 1 \\
\hline $120-125$ & 25 & 0 & 0 & 0 & 0 & 1 \\
\hline
\end{tabular}


Assuming that there will be no repair or rehabilitation done to the pipeline, the performance value can be calculated and plotted against time in year as shown in Figure 6.2.

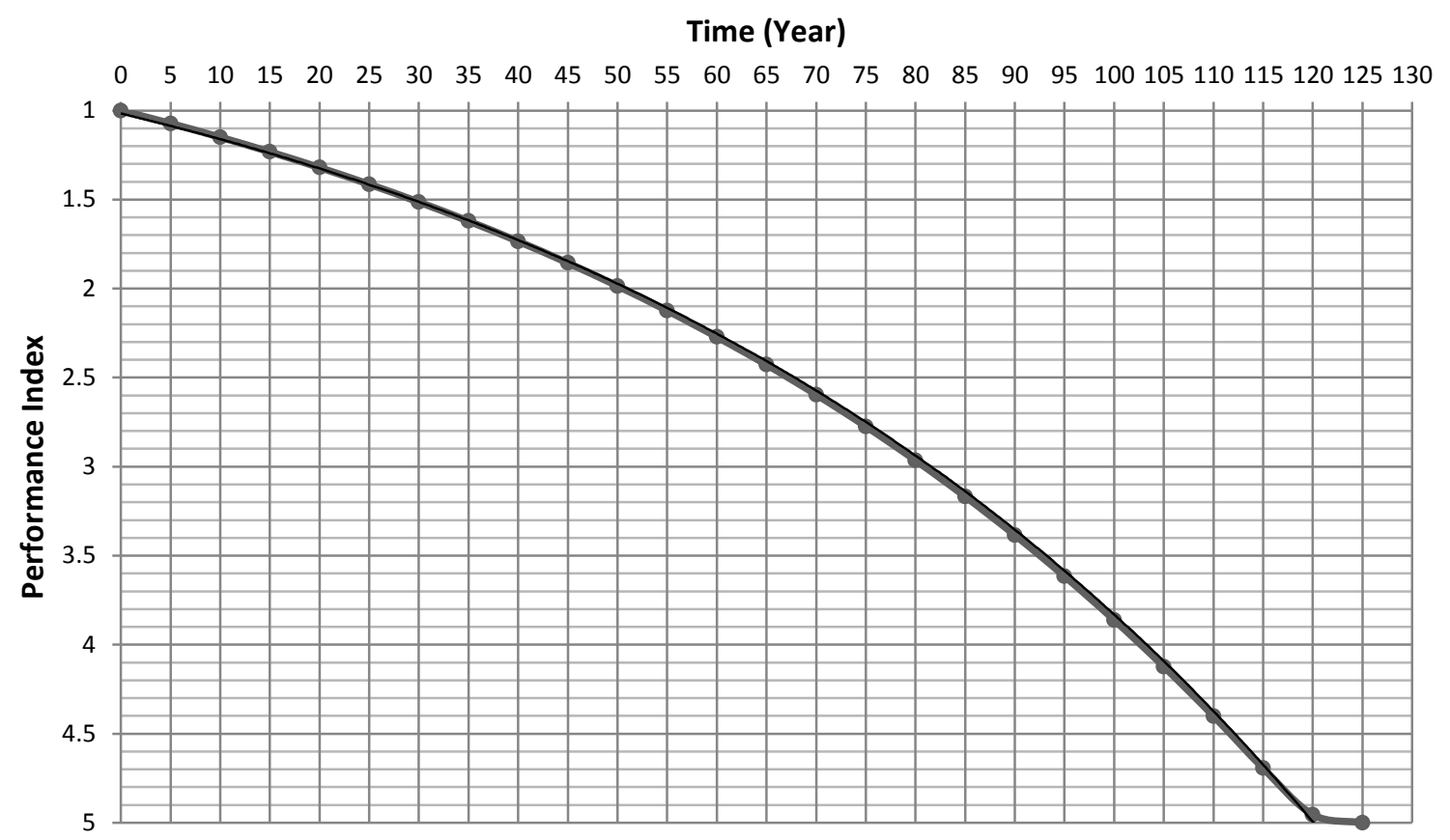

Figure 6.2 Performance Prediction for Vitrified Pipe

In this example, we forecasted the performance of the concrete and vitrified clay pipes with the diameter less than 15 inches. Implementing the expected value method and assuming the five year zoning concept, the transition probabilities were determined. Based on the performance prediction curves, we can estimate that the performance value of concrete pipes and vitrified clay pipes located in Broad Creek Basin will move to "5" (failed) at the age of 110 years old and 125 years old respectively, assuming that there will be no rehabilitation work performed (run to failure). This procedure can be used in comparing the performance prediction curves for various subcategories of the wastewater pipes. Other subcategories may include location, size, diameter, $\mathrm{H}_{2} \mathrm{~S}$ level and soil condition.

The rehabilitation planning should be based on the level of service. For example, if the acceptable level of service is set to "3", the pipes should be rehabilitated approximately every 70 years for concrete pipe as shown in figure 6.3 and every 80 years for clay pipe. 


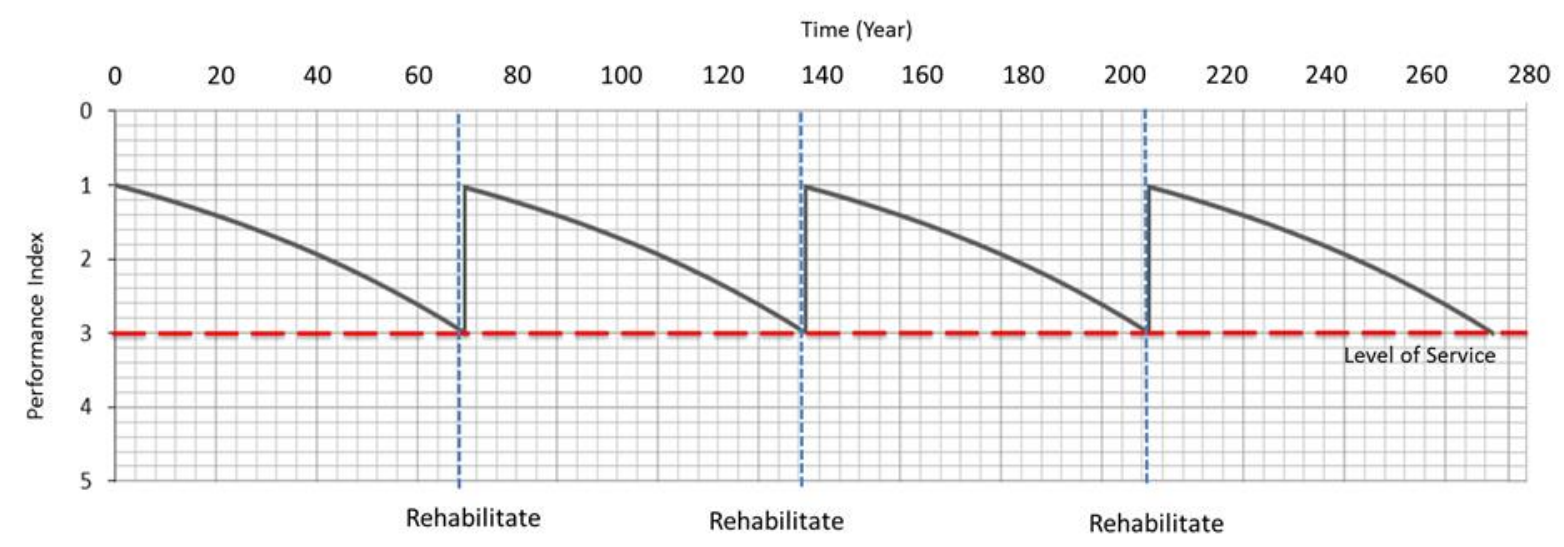

Figure 6.3 Rehabilitation Planning

In this chapter, we demonstrate that the performance index developed in the previous chapter can be linked to performance prediction using a Markov chain method which is a process widely used in the field of infrastructure. The prediction curve is crucial in estimating the overall performance of the wastewater pipes and sequentially help in inspection and renewal planning. 


\section{CHAPTER 7}

\section{CONCLUSIONS AND RECOMMENDATIONS}

This chapter presents the finding and conclusion of the work conducted in this research, and provides recommendations for future research.

\subsection{Conclusions}

In this research, the life cycle of wastewater pipeline and the causes of pipe failure in different phases including design, manufacture, construction, operation and maintenance, and repair/rehabilitation/replacement were identified as results from extensive literature reviews, various interviews with utilities, and pipe associations. The standard wastewater data structure and wastewater data collection methodologies that would enable effective and systematic data collection and data storage were proposed. Utilities and/or researchers who collect the wastewater pipe infrastructure data can utilize these data structure and data collection methodologies as a standard for establishing asset management program, developing models, or studying wastewater pipe infrastructure systems.

The performance model is a robust performance rating system created to evaluate wastewater pipes, utilizing various parameters that affect wastewater pipes as inputs. It can help 
utilities in planning, prioritization, maintenance, and repair/rehabilitation/replacement. The performance model proves to be useful in benchmarking pipe conditions and comparing the performances of pipe segments in the system and with other systems. The performance model that was developed was validated through a pilot study on utility data across the nation to test the concept and methodology.

The framework provides a noticeable improvement from the conventional practice of using solely inspection data as a mean to evaluate the wastewater pipe infrastructure system, because the model accounts for inspection data and other parameters that influence wastewater pipe performance. The fuzzy logic was good for approximate reasoning and incomplete information. We found the fuzzy inference model has many advantages over weighted factor model. The fuzzy inference model provided more sensitive results, whereas the weighted-factor model provided more static results since the weights assigned to each parameter were fixed and distributed throughout all parameters in the model. In addition, the fuzzy inference model accounted for the combination effect of dependent parameters.

The results presented were from the models built for evaluating pipes for the utilities targeted for pilot studies. As expected, some of the parameters, ranges, and weights are not applicable to all utilities. However, a generic model provides sufficient knowledge and serves as a guideline for establishing a performance evaluation program. In addition, a generic model is essential to compare results to other utilities. In case studies, where key parameters were limited, the model provided results of 0 and 1, which are excellent and good conditions, respectively. Reasonably, no model should indicate that the pipe is in bad condition without the essential parameters required for evaluation. Some of the data from were altered for security reasons. The results presented in this study serve as a filter for further and more granular and localized rehabilitation and replacement planning. The correct intervention decision should be based on a combination of specific, localized defects found within the pipe, and the economic consequences.

We recommend that utilities identify areas in which the results are problematic. These smaller areas can be analyzed in greater detail with more data, allowing additional parameters to be entered into the models. Specific data on $\mathrm{H}_{2} \mathrm{~S}$ levels, hydraulics, bedding, and soil, for example, 
can be added to the analysis. By using these smaller pilot areas, there will be a more defined initiative to gather specific data with fewer problems than using the entire system. We believe that the performance model will become more robust as utilities begin to use it and collect additional data for piloting the model.

Developing a performance index led to the development of a Markov performance prediction model. The performance prediction model utilized the performance index results from fuzzy inference model to forecast the expected performance of wastewater pipeline.

\subsection{Significances of the Research}

At present, many utilities rely on NASSCO's PACP rating system as a mean to evaluate wastewater pipes, however, the PACP rating is a defect rating and is not developed for the purpose of performance evaluation. Some utilities and consultants have created rating systems based on defect information and other parameters, yet these systems are based on only a minimal parameters with a limited methodology.

Researchers have proposed models for evaluating and predicting wastewater pipe condition and performance but only a few have been adopted by utilities due to a lack acceptance and confidence in the models. This research attempts to bridge the gap between the current practice and academia.

This research extends the fuzzy inference system application in the wastewater pipe performance evaluation area by providing fuzzy inference performance index that incorporates various factors affecting wastewater pipe performance. The fuzzy inference performance index considers all the aspects of wastewater pipe system. It evaluates the structure and function of wastewater pipes in 8 aspects including integrity, external corrosion, internal corrosion, surface wear, blockage, in/exfiltration, root intrusion, and capacity. The fuzzy inference performance index was evaluated based on a broad range of artificial, real world utility data to observe behavior of the model and to eliminate any inaccuracies, so utility can implement with only minor modifications. 
This research also contributes to the body of knowledge for wastewater pipe infrastructure by developing a standard data structure listed essential parameters affecting wastewater pipe performance, identifying failure modes and mechanisms of the wastewater pipe for all pipe material, and identifying the life cycle of wastewater pipes as results from extensive literature reviews, various interviews with utilities, and pipe associations.

The framework for wastewater pipe performance prediction was developed utilizing the performance index values. The performance prediction for specific categories can help utilities in planning inspection, planning repair and rehabilitation or making any asset management decisions.

Overall, this research has provided means and methods for wastewater pipe data collection, performance evaluation, and performance prediction. It has laid the foundation and framework for future research and development on these topics. It has enabled and encouraged the utility engineers and manager to improve their practice and collect more data for better evaluation and prediction in the future.

\subsection{Recommendations for future study}

The methodology in this research creates more interest for future research study on the topics. Future research work can be conducted by building on the findings from this research. The recommendations are summarized as follow.

1. This research identified approximately 100 parameters affecting wastewater pipe performance as presented in chapter 4, however, in developing the performance index, only 32 critical parameters were considered due to the availability of data. More parameters could be incorporated in the future research.

2. Lab testing on sample pipe sections taken from the field should be conducted to validate the performance index for all pipe materials 
3. There are many different technologies use in wastewater pipeline inspections rather than conventional CCTV, smoke test or dye test, data from these inspections can be incorporated to a performance evaluation model.

4. A performance prediction curves for all the categories varying important parameters such as material and diameter should be developed

5. More accurate performance prediction curves can be developed with the time dependent data. Different methodologies that can integrate various parameters in predicting the wastewater pipe performance could be considered.

6. The fuzzy inference performance model and performance prediction model should be utilized in inspection and Renewal decisions. 


\section{REFERNCES}

American Water Works Associations AWWA. (2005). Polyethylene Encasement for DuctileIron Pipe Systems. Denver, CO.

Abraham, D.M., Wiradhadikusumah, R., Short, T.J., and Shahbahrami, S. (1998). Optimization Modeling for Sewer Network Management, Journal of Construction Engineering and Management, vol. 124, n. 5, pp. 402-410.

American Society of Civil Engineers ASCE. (1994). State and local public work's needs, Civil Engineering Research Foundation, December.

American Society of Civil Engineers ASCE. (2000). Wastewater Facilities Construction Funding, Policy Statement 326, Reston, VA.

American Society of Civil Engineers ASCE. (2013). Report Card for America's Infrastructure, Reston, VA.

American Water Works Association AWWA. (2005). PE pipe-Design and Installation Manual of Water Supply Practices M55, ISBN 1-58321-387-2, Denver, CO.

Ana, E., Jr., and Bauwens, W. (2007). Sewer Network Asset Management Decision-Support Tools: A Review, International Symposium on New Directions in Urban Water Management, UNESCO Paris.

Ariaratnam, S. T., El-Assaly, A., and Yang, Y. (2001). Assessment of Infrastructure Inspection Needs Using Logistic Models, Journal of Infrastructure Systems. 
Bai, H., Sadiq, R.,Najjaran, H.,Rajani, B. (2008). Condition assessment of buried pipes using hierarchical evidential reasoning model, ASCE Journal of Computing in Civil Engineering, 22:2, pp. 114-122.

Baik, H., Seok, H., Jeong D., and Abraham, D. M. (2006). Estimating Transition Probabilities in Markov Chain-Based Deterioration Models for Management of Wastewater Systems, Journal of Water Resources Planning and Management.

Baur, R., and Herz, R. (2001). Selective inspection planning with aging forecast for sewer types, Proceedings IWA $2^{\text {nd }}$ World Water Congress: Efficient Water Management - Making it Happen, Berlin, Germany.

Bengassem, J. and Bennis, S. (2000). Fuzzy Expert System for Sewer Networks Diagnosis, Proceedings International Conference on Decision Making in Urban and Civil Engineering. Lyon, France. 18pp.

Black \& Veatch. (1999). Optimization of Collection System Maintenance Frequencies and System Performance, ASCE, EPA Cooperative Agreement \#CX 824902-01-0.

Bizier, P. (2007).," Gravity Sanitary Sewer Design and Construction," ASCE/EWRI/WEF.

Bonds, W. R. (1997). Stray Current Effects on Ductile Iron Pipe, Ductile Iron Pipe Research Association (DIPRA).

Boot, J.C. (1998). Elastic Buckling of Cylindrical Pipe Linings With Small Imperfections Subject to External Pressure, Journal of Intl. Society of Trenchless Technology, vol. 12, no. 2, 1998.

Brown and Caldwell. (2006). Seattle Public Utilities 2006 Wastewater Systems Plan. 
Burn, S et al. (2005). Long-Term Performance Prediction for PVC Pipes, American Water Works Association (AWWA) Research Foundation.

Butt, A. A., Shahin, M. Y., Feighan, K. J. and Carpenter, S. H. (1987). Pavement Prediction Model Using the Markov Process. Transportation Research Record (1123): 12-19.

Carnahan, J. V., Davis, W.J., Shahin, M.Y., Keane, P.L., and Wu, M.I. (1987). Optimal Maintenance Decisions for Pavement Management. Journal of Transportation Engineering, ASCE 113(5): 554-572.

Chughtai, F., and Zayed, T. (2007). Sewer Pipeline Operational Condition Prediction using Multiple Regression. Pipelines 2007: Advances and Experiences with Trenchless Pipeline Projects, ASCE.

City of Atlanta Department of Watershed Management. (n.d). Asset Management Case Study, https://courses.worldcampus.psu.edu/public/buried_assets/resources/atlanta.pdf.

Cobb County Water System CCWS. (n.d). Cobb County Water System - Homepage, http://water.cobbcountyga.gov.

Computer Aided Rehabilitation of Sewer CARE-S. (2002). European Commission - Fifth Framework Program, Technical Report EVK1-CT-2002-00106, http://care-s.unife.it.

Creighton, J. H. C. (1994). A First Course in Probability Models and Statistical Inference, Springer.

Davies, J.P., Whiter, J.J., Clark, B.A., Ockleston, G.O., and Cunningham, R.J. (1999). Application of Interaction Matrices to the Problem of Sewer Collapse, Proceedings of $11^{\text {th }}$ European Sewage and Response Symposium, Munich. 
Deb, A. K., Grablutz, F. M., Hasit, Y. J., Snyder, J. K., Loganathan, G. V., and Agbenowski, N. (2002). Prioritizing Water Main Replacement and Rehabilitation. Denver, CO, American Water Works Association Research Foundation.

DeSilva, D., Burn, S., Tjandraatmadja, G., Moglia, M., Davis, P., Wolf, L., Held, I., Vollertsen, J., Williams, W., and Hafskjold, L. (2005). Sustainable management of leakage from wastewater pipelines, Water Sci Technol. 2005; 52(12):189-98.

Ductile Iron Pipe Research Association DIPRA. (2001). Ductile Iron Pipe, DIP-GEN/3-03/5M.

Ductile Iron Pipe Research Association DIPRA. (2003). Installation Guide For Ductile Iron Pipe, ISBN 0-9642194-0-9.

Farshad, M. (2004). Two New Criteria for the Service Life Prediction of Plastics Pipes, Polymer Testing 23: 967-972.

Farshad, M. (2006). Plastic Pipe Systems: Failure Investigation and Diagnosis, Switzerland.

FCM and NRC. (2004), Assessment and Evaluation of Storm and Wastewater Collection Systems, A Best Practice by the National Guide to Sustainable Municipal Infrastructure, ISBN 1-897094-72-8, Canada.

Garcia, C., Abraham, M. D. Gokhale, S., Iseley, T. (2002), Rehabilitation Alternatives for Concrete and Brick Sewers, ASCE Practice Periodical on Structural Design and Construction.

Golroo A., P Eng., and Susan L. (2012). Development of panel rating protocol and condition evaluation model for pervious concrete pavement. Journal of Transportation Engineering, pages $315-323$.

Grigg, N. S. (2002). Water, Wastewater, and Storm Water Infrastructure Management, CRC, ISBN 1566705738. 
Grigg, N. S. (2007). Main Break Prediction, Prevention, and Control, American Water Works Association Research Foundation.

Hahn, G. J. a. S., S. S. (1994). Statistical Models in Engineering, Wiley.

Haigh, J. (2002). Probability Models. Great Britain, Springer.

Hasegawa, K., Wada, Y. and Miura, Hiroyuki. (1999). New Assessment System for Premeditated Management and Maintenance of Sewer Pipe Network, Proceedings $8^{\text {th }}$ International Conference on Urban Storm Drainage, 30 Aug - 3 Sep 1999, Sydney, Australia: 586-593.

Haykin, S. (1994). Neural Networks. New York, Macmillan College Publishing Company.

Heastad, M., Walski, M. T., Barnard, E. T., Harold, E., Merritt, B. L., Walker, N., Whitman, E. B. (2004). Wastewater Collection System Modeling and Design, First Edition, Heastad Press, Waterbury CT.

Heger, F.J., Liepins, A.A., and Selig, E.J. (1985). SPIDA: An Analysis and Design for Buried Concrete Pipe, Proceedings of the Intl. Conference of Pipelines, ASCE, pp. 143-154.

Hutchinson, E. R., El-Sayegh, K. H., and Chambers, L. (2007). Atlanta Track - Paper Two Atlanta's SSES \& Integrated Sewer Rehabilitation Selection Process, Pipelines 2007: Advances and Experiences with Trenchless Pipeline Projects, ASCE.

Jason Consultants, LLC. (2007). Inspection Guidelines for Ferrous Force Mains, Interim Report Water Environmental Research Foundation (WERF).

Jiang, Y., Saito, M., and Sinha, K.C. (1988). Bridge Performance Prediction Modeling using the Markov Chain. Transportation Research Record 1180: 25-32. 
Kaempfer, W., and Berndt, M. (1999). Estimation of service life of concrete pipes in sewer networks, Durability of Building Materials and Components, Ottawa ON, K1A 0R6, Canada, pp. 36-45.

Kathula, V. (2001). Structural Distress Condition Modeling for Sanitary Sewer, Ph.D. Thesis, Civil Engineering, Louisiana Technological University.

Kellagher R. et al. (2002). Development of Guideline for the structural Hydraulic and Environmental Rehabilitation of Sewer, SMT4 - CT98 - 2272, http://www.hydroinformatics.org/SRguide/home.html.

Khan Z., Zayed T., and Moselhi O. (2010). Structural condition assessment of sewer pipelines. Journal of Performance of Constructed Facilities, pages 170-179.

Kleiner, Y. (2001). Scheduling inspection and renewal of large infrastructure assets, Journal of Infrastructure Systems, ASCE, 7(4), pp. 136-143.

Kleiner, Y., Rajani, B., and Sadiq, R. (2004). Modeling failure risk in buried pipes using fuzzy Markov deterioration process, ASCE, International Conference on Pipeline Engineering and Construction (San Diego, CA).

Kleiner, Y., Rajani, B., and Sadiq, R. (2005). Risk Management of Large Diameter Water Transmission Mains, AWWARF, ISBN 1-58321-400-3.

Kleiner, Y., Rajani, B. and Krys, D. (2010). Impact of Soil Properties on Pipe Corrosion: ReExamination of Traditional Conventions in Water Distribution System Analysis. Tucson, AZ.

Korving, H., and van Noortwijk, M. J. (2008). Bayesian Updating of a Prediction Model for Sewer Degradation. Urban Water Journal, Vol.5 Issue 1, 51-57.

Lai, F. (2008). Review of Sewer Design Criteria and RDII Prediction Methods, United States EPA/600/R-08/010, Environmental Protection Agency (USEPA), Washington DC. 
Landau, L. J. a. T., J. G. (1998). Concepts for Neural Networks, Springer.

Li, N., Huot, M., and Haas, R. (1997). Cost-Effectiveness-Based Priority Programming of Standardized Pavement Management. Transportation Research Record: 8-16.

Lin, C. T. a. L., C. S. G. (1996). Neuro-Fuzzy Systems. Englewood Cliffs, NJ, Prentice-Hall.

Madanat, S.M., Mishalani, R., and Ibrahim, W.H. (1995). Estimation of Infrastructure Transition Probabilities from Condition Rating Data, Journal of Infrastructure Systems, no. 2, pp.120-125.

Madanat, S.M., Karlaftis, M.G., and McCarthy, P.S. (1997). Probabilistic Infrastructure Deterioration Models with Panel Data, Journal of Infrastructure Systems, vol. 3, no. 1, pp. 4-9.

Madras, N. (2002). Lectures on Monte Carlo Methods. Rhode Island, American Mathematical Society.

Makar, J.M. (2000). A Preliminary Analysis of Failures in Grey Cast Iron Water Pipes, Engineering Failure Analysis, vol. 7, pp. 43-53.

Mamdani, E. H. a. A., S. (1975). An Experiment in Linguistic Synthesis with a Fuzzy Logic Controller, International Journal of Man-Machine Studies 7(1): 1-13.

Marlow, D. e. a. (2008). A State of the Art Review: Asset Remaining Life. Initial Report, Water Environmental Research Foundation.

Martin, T., Johnson, D., Anschell, S. (2007). Using Historical Repair Data to Create Customized Predictive Failure Curves for Sewer Pipe Risk Modeling. LESAM 2nd Leading Edge Conference on Strategic Asset Management, Lishbon, Portugal.

Mauch, M. a. M., S. (2000). Stochastic Duration Models of Bridge Deck Deterioration, 79th Transportation Research Board Meeting. Washington, D. C. 
Mehle, J.J., O'Keefe, S.M. and Wrase, P.E. (2001). An Examination of Methods for Condition Rating of Sewer Pipes, Master of Science in Infrastructural Systems Engineering, University of Minnesota.

Mehta, C. R. (2006). Identification and Characterization of Parameters for Sewer Pipe Condition Rating, Pennsylvania State University. MS.

Micevski, T., Kuczera, G., and Coombes, P. (2002). Markov Model for Storm Water Pipe Deterioration, Journal of Infrastructure Systems.

Mitrani, I. (1998). Probabilistic Modeling. Cambridge, Cambridge University Press.

Mooney, C. Z. (1997). Monte Carlo Simulation. Iowa City, Sara Miller McCune.

Moser, A. P. (2001). Buried Pipe Design, Second Edition, The Mc-Graw Hill Companies Inc, NY.

Murthy, D. N. P., Xie, M., and Jiang, R. (2004). Weibull Models, Wiley.

Najafi, M. (2005). Trenchless Technology-Pipeline and Utility Design, Construction, and Renewal, Water Environment Federation (WEF Press), ISBN 0-07-142266-8.

Najjaran, H., Rajani, B., Sadiq, R. (2004) A Fuzzy expert system for deterioration modeling of buried metallic pipes, NAFIPS International Conference, Banff, AB., June 27-30, pp. 1-6.

NASSCO (2003). Pipeline Assessment and Certification Program (PACP) Reference Manual, Pikeville, MD.

National Clay Pipe Institute NCPI. (2004). Clay Pipe Handbook, WI. 
Niederreiter, H. (1992). Random Number Generation and Quasi-Monte Carlo Methods.

Montpelier, Society for Industrial and Applied Mathematics.

NRC-CNRC. (2003). Deterioration and Inspection of Water Distribution Systems, A Best

Practice by the National Guide to Sustainable Municipal Infrastructure, Canada.

NRC-CNRC. (2009). Failures in Gray Cast Iron Distribution Pipes, http://irc.nrccnrc.gc.ca/ui/bu/grayindex_e.html.

Orange County Sanitation District OCSD. (2006). Asset Management Plan 2006, http://www.ocsd.com/civica/filebank/blobdload.asp?BlobID=5818.

Pace D.K. (2004). Modeling and Simulation Verification and Validation Challenges. Johns Hopkins Apl. Technical Digest, pages 163-172.

Park, T. (2009). A Comprehensive Asset Management System for Sewer Infrastructures, Civil and Environmental Engineering. State College, PA, Pennsylvania State University. Ph.D.

Pittsburgh Water and Sewer Authority PWSA. (2003). Monitoring \& Sampling Plan, PWSA CSO Long-Term Control Plan Development Project, https://courses.worldcampus.psu.edu/public /buried_assets/resources/pittsburgh.pdf.

Pittsburgh Water and Sewer Authority PWSA. (n.d). Pittsburgh Water and Sewer Authority Homepage, http://www.pgh2o.com/.

Rahman, S.; and Vanier, D.J. (2004). An Evaluation of Condition Assessment Protocols for Sewer Management, NRC-CNRC B-5123.6.

Rajani, B.B., Zhan, C., and Kuraoka, S. (1996). Pipe-Soil Interaction Analysis of Jointed Water Mains, Canadian Geotechnical Journal, vol. 33, no. 3, pp. 3-11. 
Rajani et al. (2000). Investigation of Grey Cast Iron Water Mains to Develop a Methodology for Estimating Service Life, American Water Works Association (AWWA) Research Foundation.

Reed, C., Robinson, J. A., Smart, D. (2004). Techniques for Monitoring Structural Behaviour of Pipeline Systems: Subject Area: Infrastructure Reliability, American Water Works Association (AWWA) Research Foundation, ISBN 1583213309.

Reed, C., Robinson, J. A., and Smart, D. (2006). Potential Techniques for the Assessment of Joints in Water Distribution Pipelines, American Water Works Association (AWWA) Research Foundation.

Rinne, H. (2009). The Weibull Distribution. Boca Raton, CRC Press.

Ross \& Associates Environmental Consulting, Ltd. (2005). Managing for Excellence: Analysis of Water and Wastewater Utility Management Systems, EP-W-04-023, The United States Environmental Protection Agency (U.S.EPA).

Ross, S. M. (1972). Introduction to Probability Models. New York, Academic Press.

Ross, T. J. (1995). Fuzzy Logic with Engineering Applications, McGraw-Hill.

Rothman, P.S., Price, R.E. (1986). Detection and Considerations of Corrosion Problems of Prestressed Concrete Cylinder Pipe, ASTM STP906-EB, Corrosion Effect of Stray Currents and the Techniques for Evaluating Corrosion of Rebars in Concrete, Philadelphia, PA.

Rothschild, V. a. L., N. (1986). Probability Distributions, John Wiley and Sons, Inc.

Ruwanpura, J., Ariaratnam, S.T. and El-Assaly, A. (2004). Prediction Models for Sewer Infrastructure Utilizing Rule-Based Simulation, Civil Engineering and Environmental Systems, 21(3): 169-185. 
Sargent R. (2007). Verification and validation of simulation models. Proceedings of the 2007 Winter Simulation Conference.

Schlesinger S. (1979). Terminology for Model Credibility. Simulation, Vol. 32, No. 3.

Schrock, B.J. (1991). Pipeline Systems Rehabilitation Workshop, San Jose, CA.

Serpente, R.F. (1993). Understanding the Models of Failure for Sewers, Proceedings of Intl. Conference of Pipeline Division, ASCE, New York, p. 86-100.

Shah, A., Tighe, S., and Stewart, A. (2004). Development of a Unique Deterioration Index, Prioritization Methodology, and Foreign Object Damage Evaluation Models for Canadian Airfield Pavement Management. Canadian Journal of Civil Engineering, 31(4): 608-618.

Shamir, U., and Howard, D. (1979). An Analytic Approach to Scheduling Pipe Replacement, Journal of the American Water Works Association, May, pp. 248-258.

Siler, W. a. B., J. J. (2005). Fuzzy Expert Systems and Fuzzy Reasoning, John Wiley \& Sons, Inc.

Sinha, S. K. a. M., R. A. (2007). Probabilistic Based Integrated Pipeline Management System. Tunneling and Underground Space Technology 22(5-6): 543-552.

Sivanandam, S. N., Sumathi, S. and Deepa, S. N. (2007). Introduction to Fuzzy Logic using MATLAB. Heidelberg, Springer-Verlag Berlin Heidelberg.

Smith, L.A., Fields, K. A., Chen, A. S. C., and Tafuri, A. N. (2000). Options for Leak and Break Detection and Repair of Drinking Water Systems, Columbus, OH: Battelle Press. 
Smith, T.E. (1994). Analysis of Tidal Fluctuation Effects on a Confined and Unconfined Aquifer, in FOCUS Conference on Eastern Regional Ground Water Issues. Newburyport, MA. p. 757-771.

Stathis, J.A. (1998). A Model for Determining Leakage in Water Distribution Systems, in Civil Engineering. Virginia Polytechnic and State University: Blacksburg.

Sugeno, M. (1985). Industrial Applications of Fuzzy Control, Elsevier Science.

Tabesh, M., and Madani, S. (2006) A Performance Indicator for Wastewater Collection. Water Practice \& Technology Vol.1 No.4.

Tafuri, N.A, and Selvakumar, A. (2001). Wastewater collection system infrastructure research needs in the USA, US Environmental Protection Agency, Urban Water 4 21-29, Edison, NJ.

Tran, D.H., Ng, A.W.M., Perera, B.J.C. (2007). Neural networks deterioration models for serviceability condition of buried stormwater pipes, Engineering Applications of Artificial Intelligence.

U.I. (1981). Capital infrastructure investment needs and financing options, HUD-0003404, Department of Housing and Urban Development, Washington, DC.

U.S.EPA. (1992). Guidelines for Water Reuse, EPA/625/R-92/004, US EPA Office of Technology Transfer and Regulatory Support.

U.S.EPA. (1998). Water Recycling and Reuse: The Environmental Benefits, IX - EPA 909-F-98001, US EPA Water Division Region.

U.S.EPA. (2000). Wastewater Technology Fact Sheet Pipe Construction and Materials, EPA 832-F-00-068, Office of Water, Washington, DC. 
U.S.EPA. (2001). Preamble of the Draft Proposed SSO Rule.

U.S.EPA. (2002). The Clean Water and Drinking Water Infrastructure Gap Analysis, EPA-816R-02-020, Office of Water (4606M).

U.S.EPA. (2005). Managing for Excellence: Analysis of Water and Wastewater Utility Management Systems, Washington, D.C.

Uni-Bell. (2001). Handbook of PVC Pipe Design and Construction, Dallas, TX.

Urquhart, T. (2000). Protocols for Assessing Condition and Performance of Water and Wastewater Assets. Alexandria, VA, Water Environment Research Foundation.

Urquhart, T., Burn, S. (2006). Protocols for Assessing Condition and Performance of Water and Wastewater Assets, Draft Final Report WERF Project 03-CTS-20CO, Water Environmental Research Foundation (WERF) Condition Assessment Protocols (CAP) Project.

Water Infrastructure Network WIN. (2000). Clean and Safe Water for the 21st Century, Washington, D.C.

Water Research Center WRc (2001). Sewer Rehabilitation Manual.

Whidden, R.W. (2009). Buried Flexible Steel Pipe Design and Structural Analysis. ASCE Manuals and Reports on Engineering Practice No. 119, Reston, VA: American Society of Civil Engineers.

Whittle, A. J.; Tennakoon, J. (2005). Predicting the residual life of PVC sewer pipes, Plastics, Rubber and Composites, Volume 34, Number 7, pp. 311-317(7). 
Wienhold, P., Chudnovsky, A. (2006). Lifetime of High-Density Polyethylene Drain Pipe in an Aggressive Environment, Transportation Research Board Annual Meeting 2006 Paper \#06-2377, Washington, DC.

Wiley \& Wilson. (2006). Town of Blacksburg Sanitary Sewer System Study, VA.

Wirahadikusumah, R., Abraham, D., and Iseley, T. (2001). Challenging Issues in Modeling Deterioration of Combined Sewers, Journal of Infrastructure Systems.

Zadeh, L. A. (1965). Fuzzy Sets. Information and Control 8: 338-353. 


\section{APPENDIX A}

\section{Pipe Life CyCle}




\section{Table A-1. Concrete Wastewater Pipe Life Cycle}

\section{Failure Causes}

\section{Design}

1. Poor Design

Connection, Vertical connections, Mechanical vulnerable material, Chemical vulnerable material, Trust restraint, Corrosion control, Thickness, Special applications

2. Poor Project Planning Improper connections, Under-design for load, Time limited design, Poor assumptions of environment

3, Dimensioning

\section{Manufacture}

4. Manufacturing Defects

Wall thickness, Inclusions of unintended structures, Porosity due to air pockets, Longitudinal surface defects, Cheap composite material, Improper connections

\section{Storage}

6. Observation and Quality Control

\section{Construction}

7. Transit

Transit damage

8. Human Error

Third party damage

9. Poor Workmanship

Improper connections, Uneven bedding, Poor backfilling Material, impact damage, Lining issues, Poor joint

10. Observation and Quality Control

\section{Operation \& Maintenance}

\section{Mechanical}

12. Thermal

13. Chemical

14. Biological

15. External Interference

16. Internal Interference

17. Natural Catastrophes

18. Inappropriate Service

19. Inappropriate Maintenance
Material properties, Hydraulic factors

Temperature failures

Chemical attacks

Biological attacks

Movement of soil, Tensile and compression failures

Fatigue 


\section{Repair/Rehabilitation/Replacement}

20. Design Error

21. Manufacturing Error

22. Construction Error

23. Operation \& Maintenance Error 
Table A-2.Clay Wastewater Pipe Life Cycle

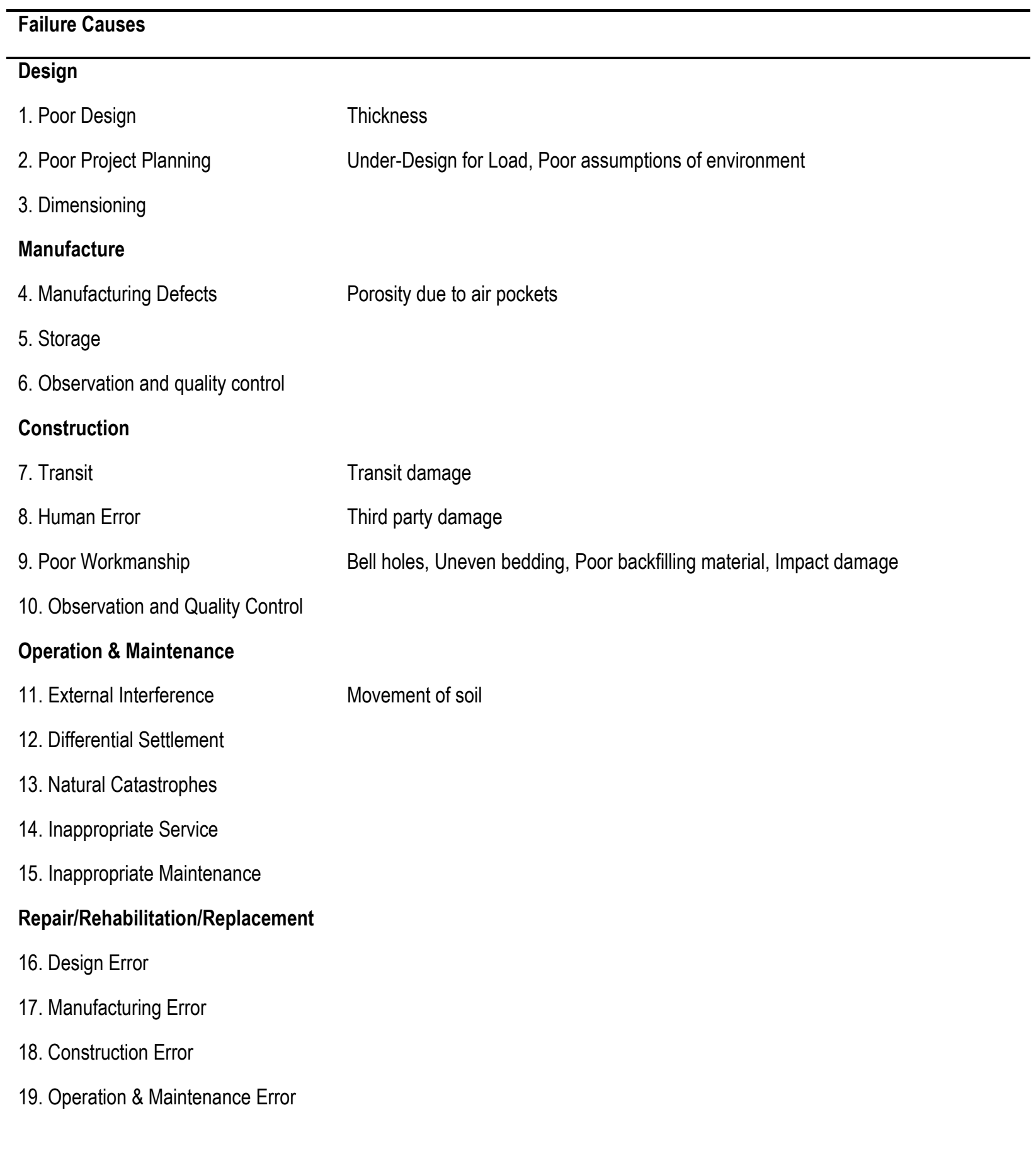


Table A-3. Ductile Iron Wastewater Pipe Life Cycle

\section{Failure Causes}

\section{Design}

1. Poor Design

Coating, connection, Vertical connections, Chemical vulnerable material, Thrust restraint, Corrosion control, Thickness, Special applications

2. Poor Project Planning Improper connections, Under-design for load, Time limited design, Poor assumptions of environment

3. Dimensioning

\section{Manufacture}

4. Manufacturing Defects

Wall thickness, Inclusions of unintended structures, Surface defects, Cheap composite material, Improper connections

5. Storage

6. Observation and Quality Control

\section{Construction}

7. Transit

Coating damage

8. Human Error

Third party damage

9. Poor Workmanship

Improper connections, Uneven bedding, Poor backfilling material, Impact damage, Lining issues

10. Observation and Quality Control

Operation \& Maintenance

11. Mechanical

12. Thermal

13. Chemical

14. Biological

15. External Interference

16. Internal Interference

17. Natural Catastrophes

18. Inappropriate Service

19. Inappropriate Maintenance
Material properties, Hydraulic factors

Temperature failures

Pitting corrosion, Galvanic corrosion, Graphitization

Microbiologically influenced corrosion

Dissimilar electrolytes, Stray current corrosion, Movement of soil, Tensile and compression failures

Fatigue 


\section{Repair/Rehabilitation/Replacement}

20. Design Error

21. Manufacturing Error

22. Construction Error

23. Operation \& Maintenance Error 
Table A-4. Grey Cast Iron Wastewater Pipe Life Cycle

\section{Failure Causes}

\section{Design}

1. Poor Design

Coating, Connection, Vertical connections, Mechanical vulnerable material, Chemical vulnerable material, Trust restraint, Corrosion control, Thickness, Special applications

2. Poor Project Planning Improper connections, Under-design for load, Time limited design, Poor assumptions of environment

3. Dimensioning

\section{Manufacture}

4. Manufacturing Defects

Wall thickness, Inclusions of unintended structures, Porosity due to air pockets, Longitudinal surface defects, Cheap composite material, Pipe material content, Improper connections

\section{Storage}

6. Observation and Quality Control

\section{Construction}

7. Transit

Coating damage

8. Human Error

Third party damage

9. Poor Workmanship

Improper connections, Uneven bedding, Poor backfilling material, Impact damage, Lining issues

10. Observation and Quality Control

\section{Operation \& Maintenance}

11. Mechanical

12. Thermal

13. Chemical

14. Biological

15. External Interference

16. Internal Interference

17. Natural Catastrophes

18. Inappropriate Service

19. Inappropriate Maintenance
Material properties, Hydraulic factors

Temperature failures

Pitting corrosion, Galvanic corrosion, Graphitization

Microbiologically influenced corrosion

Dissimilar electrolytes, Stray current corrosion, Movement of soil, Tensile and compression failures

Fatigue 


\section{Repair/Rehabilitation/Replacement}

\section{Design Error}

21. Manufacturing Error

22. Construction Error

23. Operation \& Maintenance Error 


\section{Table A-5.PVC Wastewater Pipe Life Cycle}

\section{Failure Causes}

\section{Design}

1. Poor Design

Connection, Vertical connections, Trust restraint, Thickness, Special applications

2. Poor Project Planning

Improper connections, Under-design for load, Time limited design, Poor assumptions of environment

3. Dimensioning

\section{Manufacture}

4. Manufacturing Defects

Wall Thickness, Inclusions of unintended structures, Porosity due to air pockets, Surface defects, Cheap composite material, Improper connections

\section{Storage}

6. Observation and Quality Control

\section{Construction}

7. Transit

Transit Damage

8. Human Error

Third Party Damage

9. Poor Workmanship

Improper connections, Uneven bedding, Poor backfilling material, Impact damage, Improper trench

10. Observation and Quality Control

\section{Operation \& Maintenance}

$\begin{array}{ll}\begin{array}{l}\text { 11. Mechanical } \\ \text { 12. Thermal }\end{array} & \text { Material properties, Hydraulic factors } \\ \text { 13. Chemical } & \text { Temperature failures } \\ \text { 14. Biological } & \text { Chemical attacks } \\ \text { 15. External Interference } & \text { Mological attacks } \\ \text { 16. Internal Interference } & \text { Fatigue } \\ \text { 17. Natural Catastrophes } & \\ \text { 18. Inappropriate Service } & \\ \text { 19. Inappropriate Maintenance }\end{array}$




\section{Repair/Rehabilitation/Replacement}

\section{Design Error}

21. Manufacturing Error

22. Construction Error

23. Operation \& Maintenance Error 
Table A-6. PE and HDPE Wastewater Pipe Life Cycle

\section{Failure Causes}

\section{Design}

1. Poor Design

2. Poor Project Planning

3. Dimensioning

\section{Manufacture}

4. Manufacturing Defects

5. Storage

6. Observation and Quality Control

\section{Construction}

7. Transit

8. Human Error

9. Poor Workmanship

10. Observation and Quality Control

Operation \& Maintenance

11. Mechanical

12. Thermal

13. Chemical

14. Biological

15. External Interference

16. Internal Interference

17. Natural Catastrophes

18. Inappropriate Service

19. Inappropriate Maintenance
Connection, Vertical connections, Thrust restraint, Thickness, Special applications

Improper connections, Under-design for load, Time limited design, Poor assumptions of environment

Wall thickness, Inclusions of unintended structures, Porosity due to air pockets, Surface defects, Cheap composite material, Improper connections

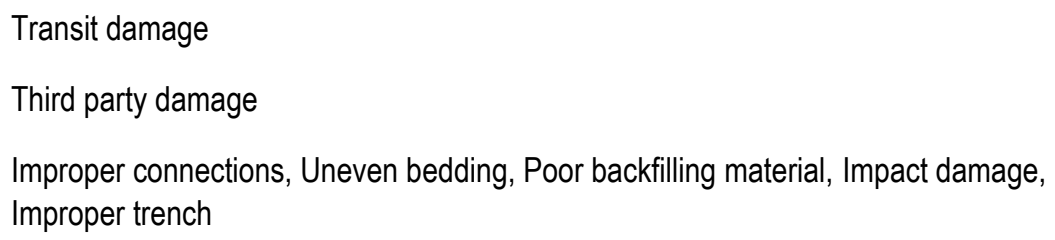

Transit damage

Third party damage

Improper connections, Uneven bedding, Poor backfilling material, Impact damage, Improper trench

Material properties

Temperature failures

Chemical attacks

Biological attacks

Movement of soil, Tensile and compression failures

Fatigue 


\section{Repair/Rehabilitation/Replacement}

20. Design Error

22. Manufacturing Error

23. Construction Error

24. Operation \& Maintenance Error 
Table A-7. Brick Sewers Life Cycle

\author{
Failure Causes \\ Design \\ 1. Poor Design \\ 2. Poor Project Planning \\ 3. Dimensioning \\ Construction \\ 4. Human Error \\ Third party damage \\ 5. Poor Workmanship \\ Uneven bedding, Impact damage \\ 6. Observation and Quality Control \\ Operation \& Maintenance \\ 7. Thermal \\ Temperature failures \\ 8. Chemical \\ Chemical attacks \\ 9. Biological \\ Biological attacks \\ 10. External Interference \\ Movement of soil, Tensile and compression failures \\ 11. Natural Catastrophes \\ 12. Inappropriate Service \\ 13. Inappropriate Maintenance \\ Repair/Rehabilitation/Replacement \\ 14. Design Error \\ 15. Manufacturing Error \\ 16. Construction Error \\ 17. Operation \& Maintenance Error
}

Mechanical vulnerable material, Chemical vulnerable material, Wall thickness

Under-design for load, Time limited design, Poor assumptions of environment 


\section{APPENDIX B}

\section{FAILURE MODE AND MECHANISM}




\section{Table B-1. Failure Modes of CP Wastewater Pipe}

\section{CP Wastewater Pipes Failure Modes}

\section{A. Main Line}

\section{Typical Failure Mode}

\section{Structural Failure}

1 Crack

2 Fracture

3 Broken

$4 \quad$ Hole

5 Deformed

$6 \quad$ Collapsed

$7 \quad$ Surface damage

$8 \quad$ Lining Failure
Longitudinal crack, Circumferential crack, Multiple cracks

Longitudinal fracture, Circumferential fracture, Multiple fractures

Vertical deformed, Horizontal deformed

Roughness increased, Aggregate visible, Aggregate projecting, Aggregate missing, Missing wall, Surface spalling

Defective Weld, Detached, Defective end, Blistered, Service cut shifted, Abandoned connection, Overcut service, Undercut service, Buckled, Wrinkled

\section{Operational and Maintenance Failure}

9 Deposits

10 Roots

11 Infiltration

12 Exfiltration

13 Obstacles/Obstructions

\section{Rare Failure Mode}

\section{Structural Failure}
14 Crack
Spiral crack
15 Fracture
Spiral fracture obstacles
Attached, Settled, Ingress

Fine, Tap, Medium, Ball

Weeper, Dripper, Runner, Gusher

Pipe material in invert, Object intruding through wall, Object wedged in the joint, Object through connection, External pipe or cable, Construction debris, Rocks, Other 


\section{B. Joint}

\section{Typical Failure Mode}

$1 \quad$ Lined Joint

Separated Joint

2 Rubber Gasket Joint

Corrosion degradation of reinforcing wires, Gasket degradation, Concrete degradation, Mortar seal degradation, Seal displacement

3 Steel Endring Joint

Weld failure, Corrosion of spigot, Ovality of pipe 
Table B-2. Failure Modes of RCP Wastewater Pipe

RCP Wastewater Pipes Failure Modes

A. Main Line

Typical Failure Mode

Structural Failure

1 Crack

2 Fracture

3 Broken

4 Hole

5 Deformed

6 Collapsed

$7 \quad$ Surface damage

$8 \quad$ Lining Failure
Longitudinal crack, Circumferential crack, Multiple cracks

Longitudinal fracture, Circumferential fracture, Multiple fractures

Vertical deformed, Horizontal deformed

Roughness increased, Aggregate visible, Aggregate projecting, Aggregate missing, Reinforcement visible, Reinforcement projecting, Reinforcement corroded, Missing wall, Surface spalling

Defective Weld, Detached, Defective end, Blistered, Service cut shifted, Abandoned connection, Overcut service, Undercut service, Buckled, Wrinkled

\section{Operational and Maintenance Failure}

$9 \quad$ Deposits

10 Roots

11 Infiltration

12 Exfiltration

13 Obstacles/Obstructions

Rare Failure Mode

\section{Structural Failure}
14 Crack
15 Fracture

Attached, Settled, Ingress

Fine, Tap, Medium, Ball

Weeper, Dripper, Runner, Gusher

Pipe material in invert, Object intruding through wall, Object wedged in the joint, Object through connection, External pipe or cable, Construction debris, Rocks, Other obstacles

Spiral crack

Spiral fracture 


\section{B. Joint}

\section{Typical Failure Mode}

$1 \quad$ Lined Joint

Separated Joint

2 Rubber Gasket Joint

Corrosion degradation of reinforcing wires, Gasket degradation, Concrete degradation, Mortar seal degradation, Seal displacement

3 Steel Endring Joint

Weld failure, Corrosion of spigot, Ovality of pipe 
Table B-3. Failure Modes of Clay Wastewater Pipe

\section{Clay Wastewater Pipe Failure Modes}

A. Main Line

\section{Typical Failure Mode}

\section{Structural Failure}

1 Cracks / Fractures

Longitudinal (localized) crushing failure, Star breaks, Shear failure, Lateral shear, Shear between manhole pipeline

2 Collapsed

Operational and Maintenance Failure

3 Sags in the line

4 Deposits Attached, Settled, Ingress

5 Roots Fine, Tap, Medium, Ball

6 Infiltration Weeper, Dripper, Runner, Gusher

7 Exfiltration

\section{Rare Failure Mode}

\section{Structural Failure}

8 Surface Damage

9 Abrasion

Operational and Maintenance Failure

10 Obstacles/Obstructions Pipe material in invert, Object intruding through wall, Object wedged in the joint, Object through connection, External pipe or cable, Construction debris, Rocks, Other obstacles

\section{Failure Mode}

Typical Failure Mode

1 Parallel offsets

2 Pulled Joints

3 Excessive angular

deflection

4 Joint Leakage 
Table B-4. Failure Modes of Ductile Iron Wastewater Pipe (Gravity)

Ductile Iron Wastewater Pipe Failure Modes (Gravity)

A. Main Line

Typical Failure Mode

Structural Failure

1 Hole

2 Corrosion

External corrosion, Internal corrosion

3 Deformed

Vertical deformed, Horizontal deformed

$4 \quad$ Lining Failure

Detached, Defective end, Blistered, service cur shifted, Abandoned

connection, Overcut service, Undercut service, Buckled, Wrinkled, Spall, Crack

Operational and Maintenance Failure

5 Deposits

Attached, Settled, Ingress

6 Roots

Fine, Tap, Medium, Ball

7 Infiltration

Weeper, Dripper, Runner, Gusher

8 Exfiltration

Rare Failure Mode

Structural Failure

9 Crack

Circumferential crack, Longitudinal crack, Spiral crack, Mixed crack

10 Fracture

Circumferential fracture, Longitudinal fracture, Spiral fracture, Mixed fracture, Shearing, Bell shearing

11 Collapsed

Operational and Maintenance Failure

12 Obstacles/Obstructions

Object intruding through wall, Object wedged in the joint, Object through connection, External pipe or cable, Other obstacles

\section{B. Joint}

\section{Typical Failure Modes}

1 Push-On

2 Bolted Flange

$3 \quad$ Restrained Joint
Gasket/Seal failure, Angular deflection, Axial pull out, Thermal expansion/cracked spigot, Lateral pipe movement, Internal corrosion, External corrosion

Bolt failure, Flange failure, Gasket failure, Stress at flange , Bolt corrosion

Pull out, External corrosion 


\section{Rare Failure Modes}

4 Mechanical Joint

Bolt failure, Bolt corrosion, Backing ring corrosion, Structural spigot failure

5 Slip-On Coupler

Internal corrosion, External corrosion, Bolt corrosion, Spigot pull-out

6 Ball \& Socket

Structural socket failure 
Table B-5. Failure Modes of Cast Iron Wastewater Pipe (Gravity)

Cast Iron Wastewater Pipe Failure Modes (Gravity)

A. Main Line

\section{Typical Failure Mode}

\section{Structural Failure}

1. Crack Circumferential crack, Longitudinal crack, Spiral crack, Mixed crack

2. Fracture Circumferential fracture, longitudinal fracture, Spiral fracture, Mixed fracture

3. Hole Blowout holes

4. Corrosion External corrosion, Internal corrosion

5. Lining Failure Detached, Defective end, Blistered, Service cut shifted, Abandoned connection, Overcut service, Undercut service, Buckled, Wrinkled, Spall, Crack

\section{Operational and Maintenance Failure}
6. Deposits
Attached, Settled, Ingress
7. Roots
Fine, Tap, Medium, Ball
8. Infiltration
Weeper, Dripper, Runner, Gusher
9. Exfiltration

\section{Rare Failure Mode}

\section{Structural Failure}

10. Shearing

Bell Shearing

11. Burst Failure

Burst failure (int.), Burst Failure (ext.), Burst Failure (3rd party)

12. Collapsed

Operational and Maintenance Failure

13. Obstacles/Obstructions

Object intruding through wall, Object wedged in the joint, Object through connection, External pipe or cable, Other obstacles

\section{B. Joint}
1. Mechanical Joint
Bolt failure, Bolt corrosion, Backing ring corrosion, Structural spigot failure
2. Bolted Flange
Bolt failure, Flange failure, Gasket failure, Stress at flange, Bolt corrosion
3. Slip-On Coupler
Internal corrosion, External corrosion, Bolt corrosion, Spigot pull-out
4. Groove \& Shoulder
Structural socket failure 
5. Ball \& Socket

Rare Failure Modes

6. Push-On

7. Ball \& Spigot Lead/Jute
Structural socket failure

Gasket/seal failure, Angular deflection, Axial pull out, Thermal expansion/cracked spigot, Lateral pipe movement, Internal corrosion, External corrosion

Angular deflection, Axial pull out, Lead/jute deterioration, Thermal expansion/cracked spigot, Lateral pipe movement, External corrosion 
Table B-6. Failure Modes of PVC Wastewater Pipe (Gravity)

PVC Wastewater Pipe Failure Modes (Gravity)

A. Main Line

Typical Failure Mode

Structural Failure

1 Crack

Longitudinal crack, Spiral crack, Bell splitting

2 Fracture

Longitudinal fracture, Spiral fracture

3 Broken

$4 \quad$ Hole

5 Deformed

Axial Deformed

$6 \quad$ Buckling

Axial buckling, Transverse/Ring buckling, Non-symmetric buckling,

Longitudinal buckling, Other

$7 \quad$ Color Change

$8 \quad$ Blisters/Voids

Voids

Operational and Maintenance Failure

9 Deposits Attached, Settled, Ingress

10 Roots

Fine, Tap, Medium, Ball

11 Infiltration

Weeper, Dripper, Runner, Gusher

12 Exfiltration

\section{Rare Failure Mode}

\section{Structural Failure}

13 Crack

14 Fracture

15 Crazing

16 Deformed

17 Dimension Change

18 Blisters/Voids

19 Abrasion/Erosion

20 Chemical Attacks

21 Collapse
Circumferential crack, Mixed crack, Irregular crack, Rapid Crack Propagation (RCP)

Circumferential fracture, Mixed fractures

Through crazing, External crazing, Internal crazing

Ring Deformed

External blister, Internal blister, Blazes, Delamination 


\section{Operational and Maintenance Failure}

23 Obstacles/Obstructions

Object intruding through wall, Object wedged in the joint, Object through connection, External pipe or cable, Other obstacles

\section{B. Joint}

\section{Typical Failure Mode}

$1 \quad$ Push-fit and slip-on collar (Gasket)

Displaced gasket (into socket), Cracking of socket

\section{Rare Failure Modes}

2 Push-fit and slip-on collar (Gasket)

Spigot deflection, Gasket degradation, Bell splitting, PVC material degradation

3 Solvent Weld

Poor Adhesion degradation, Embrittlement, Bell splitting, Angular deflection, Misalignment

$4 \quad$ Other

Joint leak, Mechanical joint leak, Blown rubber on flange, Blown joint, Leak on collar, Leaking rubber ring, Gasket/Seal failure, Structural bell failure, Butt fusion joint, Electro-fusion joint, Flange joint failure 
Table B-7. Failure Modes of PE and HDPE Wastewater Pipes (Gravity)

PE and HDPE Wastewater Pipes Failure (Gravity)

A. Main Line

Failure Mode

Typical Failure Mode

Structural Failure

1. Crack Longitudinal crack, Spiral crack

2. Fracture Longitudinal fracture, Spiral fracture

3. Broken

4. Hole

5. Deformed Axial

Operational and Maintenance Failure

6. Deposits Attached, Settled, Ingress

7. Roots Fine, Tap, Medium, Ball

8. Infiltration Weeper, Dripper, Runner, Gusher

9. Exfiltration

Rare Failure Mode

Structural Failure

10. Crack Circumferential crack, Mixed crack, Irregular crack

11. Fracture Circumferential fracture, Mixed fractures

12. Deformed Ring

13. Dimension Change

14. Abrasion/Erosion

15. Chemical Attacks

16. Collapse

Operational and Maintenance Failure

17. Obstacles/Obstructions Object intruding through wall, Object wedged in the joint, Object through connection, External pipe or cable, Other Obstacles 


\section{B. Joint}

\section{Typical Failure Mode}

1. Butt Fusion

Poor joint integrity, Gaps/cracks/splits/voids/dirt at weld interface, Mixed SDR materials, Lateral offset or misalignment

2. Electrofusion

Pull out in fitting, Poor joint integrity, Voids/contamination, Overheating of fusion wire

3. Compression fitting

High local stress, Pull-out, Crack, Insert size - leakage 
Table B-8. Failure Modes of PCCP Wastewater Pipe

PCCP Wastewater Pipes Failure Modes

A. Main Line

Typical Failure Mode

Structural Failure

1 Crack Longitudinal crack, Circumferential crack, Multiple cracks

2 Fracture Longitudinal fracture, Circumferential fracture, Multiple fractures

3 Broken

4 Hole

5 Deformed Vertical deformed, Horizontal deformed

6 Collapsed

7 Surface damage Roughness increased, Aggregate visible, Aggregate projecting, Aggregate missing, Reinforcement visible, Reinforcement projecting, Reinforcement corroded, Missing wall, Surface spalling

$8 \quad$ Wire Break

9 Lining Failure Defective weld, Detached, Defective end, Blistered, Service cut shifted, Abandoned connection, Buckled, Wrinkled

Operational and Maintenance Failure

10 Exfiltration

11 Obstacles/Obstructions Object intruding through wall, Object wedged in the joint, Object through connection, External pipe or cable, Other obstacles

Rare Failure Mode

Structural Failure

12 Crack Spiral crack

13 Fracture Spiral fracture

B. Joint

\section{Typical Failure Mode}

$1 \quad$ Lined Joint

Separated Joint

2 Rubber Gasket Joint

Corrosion degradation of reinforcing wires, Gasket degradation, Concrete degradation, Mortar seal degradation, Seal displacement

3 Steel Endring Joint Weld failure, Corrosion of spigot, Ovality of pipe 
Table B-9. Failure Modes of Ductile Iron Wastewater Pipe (Force Main)

Ductile Iron Wastewater Pipe Failure Modes (Force Main)

A. Main Line

\section{Typical Failure Mode}

Structural Failure

1 Hole Blowout holes

2 Corrosion External corrosion, Internal corrosion

3 Deformed Vertical deformed, Horizontal deformed

4 Lining Failure Detached, Defective end, Blistered, Service cut shifted, Abandoned connection, Buckled, Wrinkled, Spall, Crack

\section{Operational and Maintenance Failure}

5 Exfiltration

\section{Rare Failure Mode}

\section{Structural Failure}

6 Crack

$7 \quad$ Fracture

8 Shearing

9 Burst Failure

10 Collapsed

Operational and

Maintenance Failure

11 Obstacles/Obstructions
Circumferential crack, Longitudinal crack, Spiral crack, Mixed crack

Circumferential fracture, Longitudinal fracture, Spiral fracture, Mixed fracture

Bell shearing

Burst failure (Int.), Burst failure (Ext.), Burst failure (3rd party)

Object intruding Through wall, Object wedged in the joint, Object through connection, External pipe or cable, Other obstacles

\section{B. Joint}

\section{Typical Failure Modes}

1 Push-On

Gasket/Seal failure, Angular deflection, Axial pull out, Thermal expansion/cracked spigot, Lateral pipe movement, Internal corrosion, External corrosion

2 Bolted Flange

Bolt failure, Flange failure, Gasket failure, Stress at flange , Bolt corrosion

3 Restrained Joint

Pull out, External corrosion 


\section{Rare Failure Modes}

4 Mechanical Joint Bolt failure, Bolt corrosion, Backing ring corrosion, Structural spigot failure

5 Slip-On Coupler Internal corrosion, External corrosion, Bolt corrosion, Spigot pull-out

6 Ball \& Socket

Structural socket failure 
Table B-10. Failure Modes of Cast Iron Wastewater Pipe (Force Main)

Cast Iron Wastewater Pipe Failure Modes (Force Main)

A. Main Line

Typical Failure Mode

1. Crack Circumferential crack, Longitudinal crack, Spiral crack, Mixed crack

2. Fracture Circumferential fracture, Longitudinal fracture, Spiral fracture, Mixed fracture

3. Hole Blowout holes

4. Corrosion External corrosion, Internal corrosion

5. Lining Failure Detached, Defective end, Blistered, Service cut shifted, Abandoned connection, Buckled, Wrinkled, Spall, Crack

Operational and Maintenance Failure

6. Exfiltration

Rare Failure Mode

Structural Failure

7. Shearing Bell shearing

8. Burst Failure Burst failure (int.), Burst Failure (ext.), Burst failure (3rd party)

9. Collapsed

Operational and Maintenance Failure

10. Obstacles/Obstructions Object intruding Through wall, Object wedged in the joint, Object through connection, External pipe or cable, Other obstacles

B. Joint

Typical Failure Modes

1. Mechanical Joint

Bolt failure, Bolt corrosion, Backing ring corrosion, Structural spigot failure

2. Bolted Flange

Bolt failure, Flange failure, Gasket failure, Stress at flange, Bolt corrosion

3. Slip-On Coupler

Internal corrosion, External corrosion, Bolt corrosion, Spigot pull-out

4. Groove \& Shoulder

Structural socket failure

5. Ball \& Socket

Structural socket failure

\section{Rare Failure Modes}

6. Push-On

Gasket/seal failure, Angular deflection, Axial pull out, Thermal expansion/cracked spigot, Lateral pipe movement, Internal corrosion, External corrosion

7. Ball \& Spigot Lead/Jute Angular deflection, Axial pull out, Lead/jute deterioration, Thermal expansion/cracked spigot, Lateral pipe movement, External corrosion 
Table B-11. Failure Modes of PVC Wastewater Pipe (Force Main)

\section{PVC Wastewater Pipe Failure Modes (Force Main)}

A. Main Line

\section{Typical Failure Mode}

\section{Structural Failure}

1 Crack

2 Fracture

3 Broken

4 Hole

5 Deformed

$6 \quad$ Leakage/Burst

7 Color Change

8 Blisters/Voids

Operational and Maintenance Failure

9 Exfiltration

\section{Rare Failure Mode}

\section{Structural Failure}

10 Crack

11 Fracture

12 Crazing

13 Deformed

$14 \quad$ Buckling

15 Leakage/Burst

16 Dimension Change

17 Blisters/Voids

18 Abrasion/Erosion

19 Chemical Attacks

20 Collapse

21 Other
Longitudinal crack, Spiral crack, Bell splitting

Longitudinal fracture, Spiral fracture

Axial

Burst, Burst main, Burst section, Broken section, Blown section

Voids

Circumferential crack, Mixed crack, Irregular cracks, Rapid Crack Propagation (RCP)

Circumferential fracture, Mixed fractures

Through crazing, External crazing, Internal crazing

Ring

Axial buckling, Transverse/Ring buckling, Non-symmetric buckling, Longitudinal buckling, Other

Snap through, Two-sided snap Through, perforation, Pinhole

External blister, Internal blister, Blazes, Delamination

Blank ends, Blown T, ball valve, End blown off 


\section{Operational and Maintenance Failure}

22 Obstacles/Obstructions

Object intruding through wall, Object wedged in the joint, Object through connection, External pipe or cable, Other obstacles

$$
\text { B. Joint }
$$

\section{Typical Failure Mode}

1 Push-fit and slip-on collar (Gasket) Displaced gasket (into socket), Cracking of socket

\section{Rare Failure Modes}

2 Push-fit and slip-on collar (Gaske

Spigot deflection, Gasket degradation, Bell splitting, PVC material degradation

3 Solvent Weld

Poor adhesion degradation, embrittlement, Bell splitting, Angular deflection, Misalignment

$4 \quad$ Other

Joint leak, Mechanical joint leak, Blown rubber on flange, Blown joint, Leak on collar, Leaking rubber ring, Gasket/Seal failure, Structural bell failure, Butt fusion joint, Electro-fusion joint, Flange joint failure 
Table B-12. Failure Modes of Steel Wastewater Pipe (Force Main)

Steel Wastewater Pipe Failure Modes (Force Main)

A. Main Line

Typical Failure Mode

Structural Failure

1. Hole Blowout holes

2. Corrosion External corrosion, Internal corrosion

3. Deformed Vertical deformed, Horizontal deformed

4. Lining Failure Detached, Defective end, Blistered, Service cut shifted, Abandoned connection, Buckled, Wrinkled, Spall, Crack

5. Burst Failure Burst failure (int.), Burst failure (ext.), Burst failure (3rd party)

6. Weld Failure Longitudinal, Circumferential, Multiple, Spiral

\section{Operational and Maintenance Failure}

7. Exfiltration

Rare Failure Mode

Structural Failure

8. Crack Circumferential crack, Longitudinal crack, Spiral crack, Mixed crack

9. Fracture Circumferential fracture, Longitudinal fracture, Spiral fracture, Mixed fracture, Shearing, Bell shearing

10. Collapsed

Operational and Maintenance Failure

11. Obstacles/Obstructions Object intruding through wall, Object wedged in the Joint, Object through connection, External pipe or cable, Other obstacles

\section{B. Joint}

\section{Failure Mode}

\section{Typical Failure Modes}

1. Push-On

Gasket/seal failure, Angular deflection, Axial pull out, Thermal expansion/cracked spigot, Lateral pipe movement, Internal corrosion, External corrosion

2. Bolted Flange

Bolt failure, Flange failure, Gasket failure, Stress at flange, Bolt corrosion 
Rare Failure Modes

3.Mechanical joint

Bolt failure, Bolt corrosion, Backing ring corrosion, Structural spigot failure

4.Slip-On Coupler

Internal corrosion, External corrosion, Bolt corrosion, Spigot pull-out 


\section{APPENDIX C}

\section{Data Structure}




\section{Gold Standard Wastewater Data Structure (Essential Parameters)}

\begin{tabular}{|c|c|c|c|}
\hline No. & Parameter & Unit & Brief Explanation (See Attached Sheet for Detailed Explanation) \\
\hline & 1 & 2 & 3 \\
\hline \multicolumn{4}{|c|}{ Physical/Structural } \\
\hline 1 & $\begin{array}{l}\text { Node Identification } \\
\text { Number }\end{array}$ & Node & ID for each pipe segments (Manhole-Manhole) between nodes \\
\hline 2 & Pipe Material & Type & Different pipe materials deteriorate at different rates \\
\hline 3 & Pipe Diameter & Inch & Different pipe sizes may fall in different failure modes \\
\hline 4 & Pipe Age & Year & Older pipes may deteriorate faster than newer pipe \\
\hline 5 & Pipe Depth & Feet & Pipe Depth affects pipe loading and deteriorating rate \\
\hline 6 & Node Length & Feet & Length of pipe between nodes ( $\mathrm{MH}-\mathrm{MH})$ \\
\hline 7 & Pipe Wall Thickness & Inch & Wall thickness affects rupture resistance and corrosion penetration rates \\
\hline 8 & Pipe Location & Area & Some locations may receive roadway salt intrusion; urban, sub-urban, rural, costal, etc. \\
\hline 9 & Pipe Shape & Type & Different pipe shapes may result in different failure modes and deterioration \\
\hline 10 & Pipe Joint Type & Type & Some types of joints may undergo premature failure \\
\hline 11 & Function of pipe & Type & Different use of sewer may deteriorate at different rates; Combined, Sewer, Forced \\
\hline 12 & Pipe Bedding & Yes/No-Type & Inadequate bedding may cause premature pipe failure, special bedding use \\
\hline 13 & Trench Backfill & Type & Some backfill materials are more corrosive or frost susceptible \\
\hline 14 & $\begin{array}{l}\text { Construction } \\
\text { Specification }\end{array}$ & Spec. & Construction specifications; Installation Circumstances \\
\hline 15 & Pipe Slope & Gradient & $\begin{array}{l}\text { Slope affects the velocity of gravity flow and may result in different pipe deterioration } \\
\text { rates }\end{array}$ \\
\hline 16 & Design life of pipe & Year & The pipe design life in year \\
\hline 17 & Design strength of pipe & psi & the pipe design strength ( ring, longitudinal) \\
\hline 18 & Pipe Lining & Yes/No-Type & Lined pipes have higher resistance to corrosion \\
\hline 19 & Manhole Condition & Record & Manhole condition and relevant data \\
\hline \multicolumn{4}{|c|}{ Operational/Functional } \\
\hline 20 & Wastewater Quality & Record & records of wastewater quality tested including $P H, B O D, C O D$, and temperature \\
\hline 21 & Wastewater Pressure & psi & Internal water pressure affects pipe stresses and deterioration rate \\
\hline 22 & Pipe Hydraulics & Gallon/Min & Capacity of the sewage gravity conveying pipe \\
\hline 23 & Pipe Surcharging & Yes/No - Ft. & $\begin{array}{l}\text { Surcharging in gravity sewers in dry \& wet weather should be considered, head level in } \\
\text { feet }\end{array}$ \\
\hline 24 & $\begin{array}{l}\text { Operational \& } \\
\text { Maintenance Practices }\end{array}$ & Type- Level & $\begin{array}{l}\text { Poor practices can compromise structural integrity and water quality; very good, good, } \\
\text { fair }\end{array}$ \\
\hline 25 & Pipe Renewal Record & Record & All records of pipes renewal- type of renewal method \\
\hline 26 & Pipe Failure Record & Record & Record of Failure that occur, Failure mode should be specified \\
\hline 27 & Infiltration/Inflow & $\begin{array}{l}\text { Level- } \\
\text { Gal/Min }\end{array}$ & $\begin{array}{l}\text { infiltration/inflow may cause soil erosion, and increasing flow volume; Low, Med, High - } \\
\text { also gal./min. }\end{array}$ \\
\hline 28 & Exfiltration & Level & Exfiltration may cause erosion of soil and change soil loading on pipe; Low, Med, High \\
\hline 29 & Blockage/stoppage & Yes/No-Type & Blockage make the pipeline network inoperative, sewer pipe is no longer functional \\
\hline 30 & Sediments & Ton/Feet & Sediments per unit length \\
\hline 31 & Inspection record & $\begin{array}{l}\text { Yes/No- } \\
\text { Record }\end{array}$ & Record of inspection, method use, date of inspection \\
\hline
\end{tabular}




\begin{tabular}{|c|c|c|c|}
\hline No. & Parameter & Unit & Brief Explanation (See Attached Sheet for Detailed Explanation) \\
\hline & 1 & 2 & 3 \\
\hline \multicolumn{4}{|c|}{ Environmental } \\
\hline 33 & Soil Type & Type & Corrosive, expansive, \& compressible; hydrocarbons \& solvents cause deterioration \\
\hline 34 & Soil Corrosivity & Level & Condition of the soil related to pipe deteriorate; low, medium, high \\
\hline 35 & Soil Resistivity & Level & Soils with low electrical resistivity are more likely to have high corrosion rates \\
\hline 36 & Redox Potential & Level, $\mathrm{mV}$ & $\begin{array}{l}\text { Low Redox potentials are more favorable for sulfate reducing bacteria leading to } \\
\text { corrosion }\end{array}$ \\
\hline 37 & Soil Moisture Content & Percent & Moisture percentage in the soil may affect loading and pipe deterioration \\
\hline 38 & Stray Currents & Yes/No & Stray currents may cause electrolytic corrosion of metal pipes \\
\hline 39 & Groundwater Table & Feet & $\begin{array}{l}\text { affecting soil loading on the pipes and pipe deterioration rate; above, below sewer, } \\
\text { fluctuating }\end{array}$ \\
\hline 40 & Ground Cover & Type & Paved ground or vegetation cover result in different deterioration mode and rate \\
\hline 41 & $\begin{array}{l}\text { Loading Condition } \\
\text { (Dead Load) }\end{array}$ & Lbs/sq.ft. & Death load can be determined from infrastructure loading \\
\hline 42 & $\begin{array}{l}\text { Loading Condition (Live } \\
\text { Load) }\end{array}$ & ADT-Level & Live load can be determined from average daily traffic volume and railway loading etc. \\
\hline 43 & Rainfall/Precipitation & Inch/year & Rainfall in the areas should be monitored \\
\hline 44 & Climate - Temperature & ${ }^{0} \mathrm{~F}$ & Frost action in cold regions and seasonal soil water content variation in warmer regions \\
\hline 45 & Topography & Map & Topography is very important for the performance of pipes; contour maps \\
\hline 46 & Extreme Events & $\begin{array}{l}\text { Yes/No - } \\
\text { Type }\end{array}$ & Information related to extreme events \\
\hline \multicolumn{4}{|c|}{ Financial } \\
\hline 47 & Annual Capital Cost & $\$ /$ Year & Utility annual capital Cost and allocation criteria \\
\hline \multicolumn{4}{|c|}{ Others } \\
\hline 48 & Customer Complaint & Type & Complaints related to blockage, flooding, pollutions, etc. \\
\hline 49 & Chemistry & Event & Hydrogen sulphide may corrode pipe, etc. \\
\hline 50 & FOG & Yes/No & Fats, Oils, and Grease entering the sewer system \\
\hline 51 & Overall Pipe Condition & Rating & Condition of the pipe may be ranked from inspection tests; CCTV, smoke test, etc. (1-5) \\
\hline
\end{tabular}




\section{Gold Standard Wastewater Data Structure (Preferable Parameters)}

\begin{tabular}{|c|c|c|c|}
\hline No. & Parameter & Unit & Brief Explanation (See Attached Sheet for Detailed Explanation) \\
\hline & 1 & 2 & 3 \\
\hline \multicolumn{4}{|c|}{ Physical/Structural } \\
\hline 1 & Pipe Quality & Level & Imperfect pipes may deteriorate faster; poor, fair, good \\
\hline 2 & Pipe Section Length & Feet & Length of pipe section (Joint - joint) \\
\hline 3 & Pipe Vintage & Year & Pipes made at different time and place may deteriorate differently \\
\hline 4 & Pipe Lateral & Type & Some types or some materials of lateral may undergo premature failure \\
\hline 5 & Dissimilar Materials & Yes/No & Dissimilar metals/materials are more susceptible to galvanic corrosion \\
\hline 6 & Pipe Installation & Rating & Improper Installation may cause pipe damage and increase deterioration rate \\
\hline 7 & Pipe Manufacture & Record & $\begin{array}{l}\text { Defects in pipe walls produced by manufacturing errors can make pipes vulnerable } \\
\text { to failure }\end{array}$ \\
\hline 8 & Pipe Trench Width & Feet & Trench width may affects soil loading on the pipes and deterioration rate \\
\hline 9 & Pipe external Coating & $\begin{array}{l}\text { Yes/No - } \\
\text { type }\end{array}$ & external coating prevents corrosion of the pipe \\
\hline 10 & Pipe Cathodic Protection & $\begin{array}{l}\text { Yes/No - } \\
\text { type }\end{array}$ & Technique used to control the corrosion of a metal surface \\
\hline 11 & Pipe Thrust Restraint & $\begin{array}{l}\text { Yes/No - } \\
\text { Type }\end{array}$ & Inadequate restraint may increase longitudinal pipe stresses \\
\hline 12 & Lateral Connections & Record & $\begin{array}{l}\text { Condition of lateral connections and other related information such as type of } \\
\text { connection }\end{array}$ \\
\hline 13 & Pumping Station and WWTP & Record & Location of the pumping stations and wastewater treatment plants \\
\hline \multicolumn{4}{|c|}{ Operational/Functional } \\
\hline 14 & Sewer Odors & Yes/No & Solids build-ups, poor system hydraulics, flat grade, etc. \\
\hline 15 & Sewer Flooding & Yes/No & Flooding may change property of surrounding soil and loading on pipe \\
\hline 16 & Sewer Overflow (SSO/CSO) & Yes/No & Overflow may inundate surrounding soil and change loading on pipe \\
\hline 17 & Backup floodings & Number & Number of properties affected by flooding in Dry \& Wet weather \\
\hline 18 & Leakage allowance & $\%$ & Percentage of Leakage allowance \\
\hline 19 & Interruptions & Record & Interruption of wastewater collection services \\
\hline \multicolumn{4}{|c|}{ Environmental } \\
\hline 20 & Soil Disturbance & Yes/No & $\begin{array}{l}\text { Disturbance of soil may cause damage or change soil support or loading to the } \\
\text { pipe }\end{array}$ \\
\hline 21 & Runoff Rate & $\mathrm{Cu} . \mathrm{Ft} / \mathrm{Sec}$. & Excess water flow which can be caused by rainfall, evaporation, snow melting, etc. \\
\hline 22 & Non-Uniform Soil & Yes/No & $\begin{array}{l}\text { Non-uniform soil support in longitudinal axis may increase shear and bending } \\
\text { stresses }\end{array}$ \\
\hline 23 & Frost Penetration & $\begin{array}{l}\text { Yes/No- } \\
\text { depth }\end{array}$ & Soil ever frozen around the pipe, depth of penetration in feet. \\
\hline 24 & Non-Uniform Slope & Yes/No & non-uniform slope may reduce the operating performance \\
\hline 25 & Unstable Slope & Yes/No & Pipes in unstable slope may be subjected to downslope creep displacement \\
\hline 26 & Seismic Activity & Yes/No & Seismic loading may lead to pipe rupture and pressure surge \\
\hline 27 & Catchment Area (Sewershed) & Sq.Ft & Extent of area receiving the wastewater feeding a part or the totality of sewer \\
\hline 28 & Average Closeness to Trees & Feet & Average distance between sewer and trees \\
\hline 29 & Tidal Influences & Yes/No & $\begin{array}{l}\text { Sewer in Coaster area may be subjected to tidal influence affecting bedding of the } \\
\text { pipe }\end{array}$ \\
\hline 30 & Soil pH & $\mathrm{pH}$ & $\begin{array}{l}\text { Low } p H(<4) \text { means soil is acidic and likely to promote corrosion; high alkaline } \\
\text { conditions }(p H>8) \text { can also lead to high corrosion }\end{array}$ \\
\hline
\end{tabular}




\begin{tabular}{|c|c|c|c|}
\hline No. & Parameter & Unit & Brief Explanation (See Attached Sheet for Detailed Explanation) \\
\hline & 1 & 2 & 3 \\
\hline 31 & Soil Chloride & $\%$ & $\begin{array}{l}\text { Mortar coating usually creates a pH environment of }>12.4 \text {. Low chloride levels in } \\
\text { high } \mathrm{pH}(>11.5) \text { environments can lead to serious corrosion }\end{array}$ \\
\hline 32 & Soil Sulfate & $\%$ & $\begin{array}{l}\text { Accounts for microbial induced corrosion (MIC) and possible food source for sulfate } \\
\text { reducting bacteria in anaerobic conditions under loose coatings }\end{array}$ \\
\hline 33 & Soil Sulfide & $\%$ & Sulfate reducing bacteria giving off sulfides which are excellent electrolytes \\
\hline 34 & Pipe Connections & Type & Type of connections - Residential, Industrial, Commercial, Agricultural, others \\
\hline \multicolumn{4}{|c|}{ Financial } \\
\hline 35 & Annual Maintenance Cost & $\$ /$ Year & Routine Cleaning, etc.; Method and Cost of Maintenance \\
\hline 36 & $\begin{array}{l}\text { Annual Repair/Rehabilitation } \\
\text { Cost }\end{array}$ & $\$ /$ Year & Method and Cost of Preservation and Improvement like grouting, lining, etc. \\
\hline 37 & $\begin{array}{l}\text { Installed and Replacement } \\
\text { Cost }\end{array}$ & $\$$ & Original cost of installation and replacement cost \\
\hline 38 & Annual Operational Cost & $\$ /$ Year & cost spent each year for operating and functioning sewer system \\
\hline 39 & Annual Energy Cost & $\$ /$ Year & Cost of energy use in sewer system i.e. Forced Main, Pumping station etc. \\
\hline 40 & Depreciated Value & $\%$ & Depreciated value and method of calculation \\
\hline 41 & Benefit/Cost & Record & Benefit-Cost Analysis \\
\hline \multicolumn{4}{|c|}{ Others } \\
\hline 42 & Density of Connections & $\begin{array}{l}\text { Number/ } \\
\text { Mile }\end{array}$ & Number of properties connected to the sewer per mile \\
\hline 43 & Resident Population Served & Number & Total population living in the area that is responsibility of the system \\
\hline 44 & Failing Utilities & Yes/No & Failing wastewater or water pipes in a close proximity of the system \\
\hline 45 & Consequence/Risk & Level & Consequence of failures: low, medium, high \\
\hline 46 & Third Party Damage & Yes/No & Information related to third party damage \\
\hline 47 & Other Information & - & Information relevant for pipe condition assessment and deterioration modeling \\
\hline
\end{tabular}




\section{APPENDIX D}

\section{MEMBERSHIP FUNCTIONS}



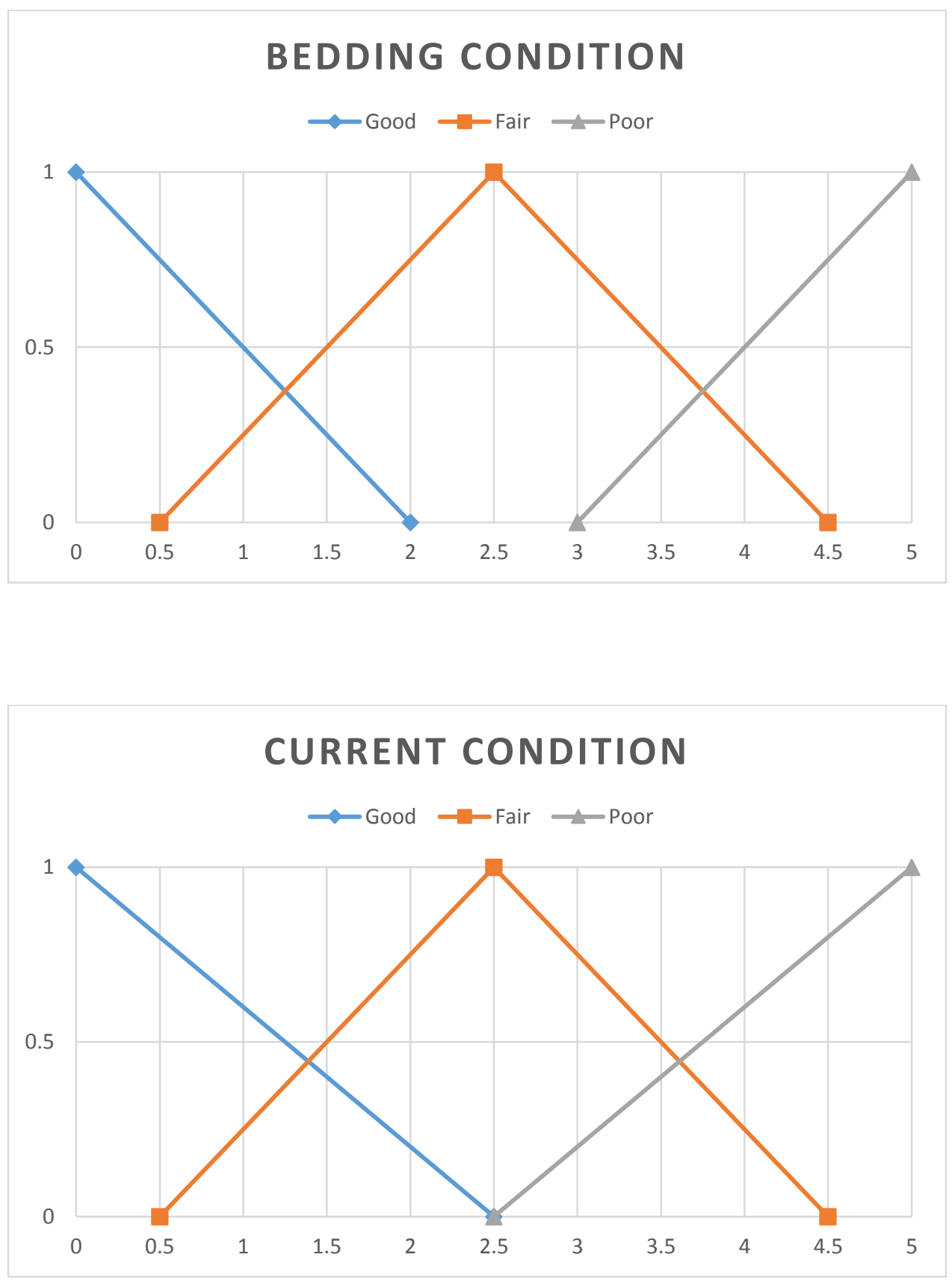

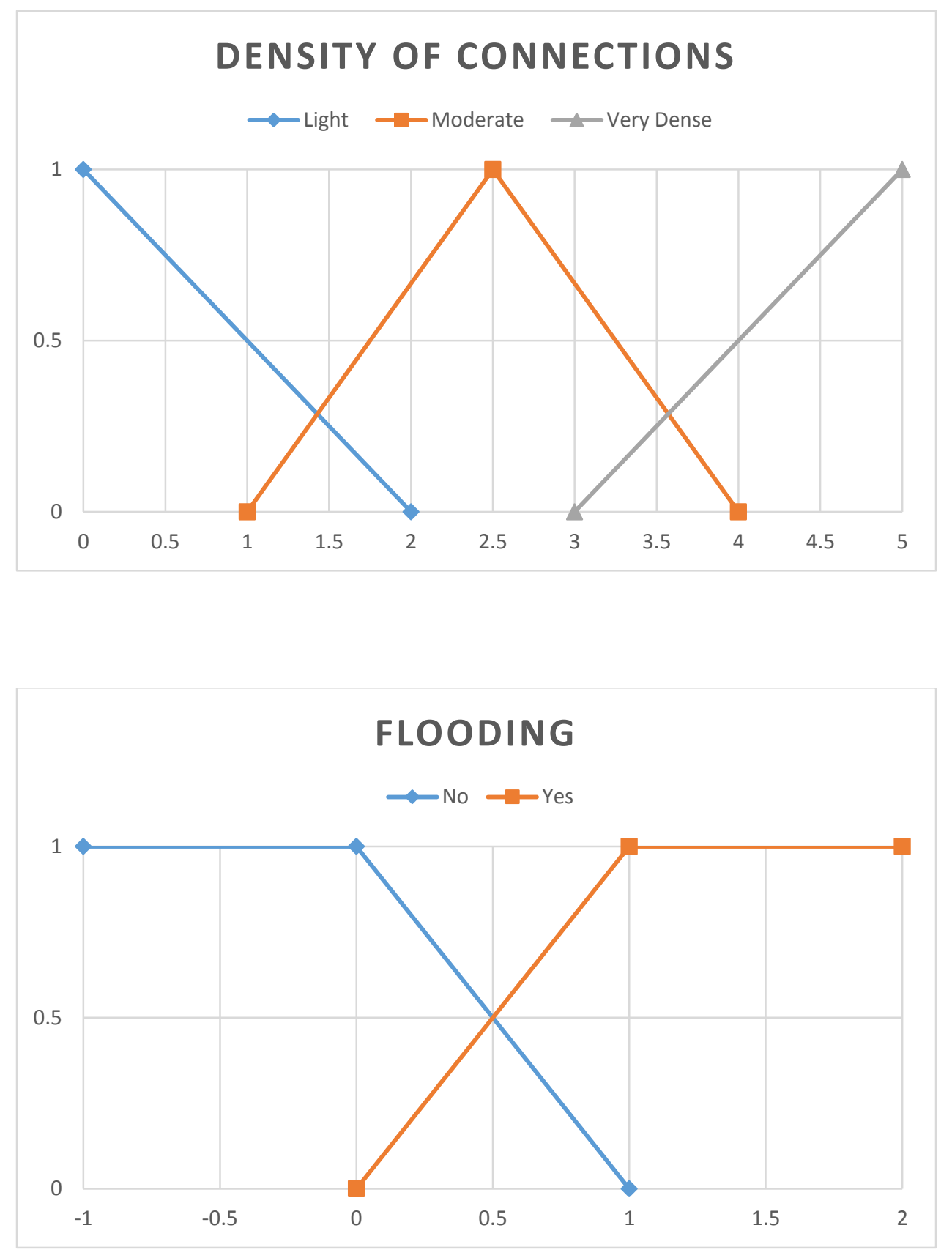


\section{FLOW DEPTH/DIAMETER}
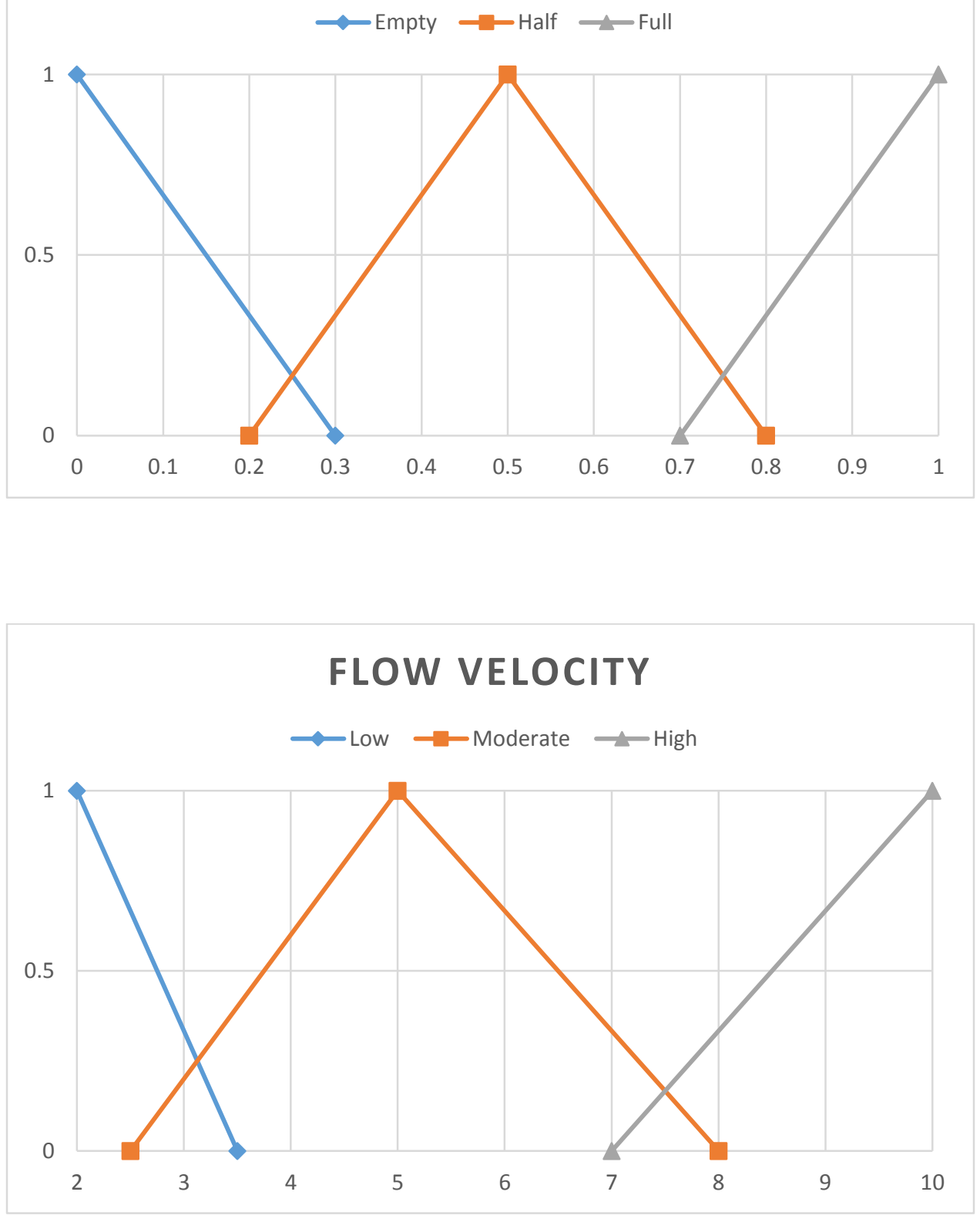

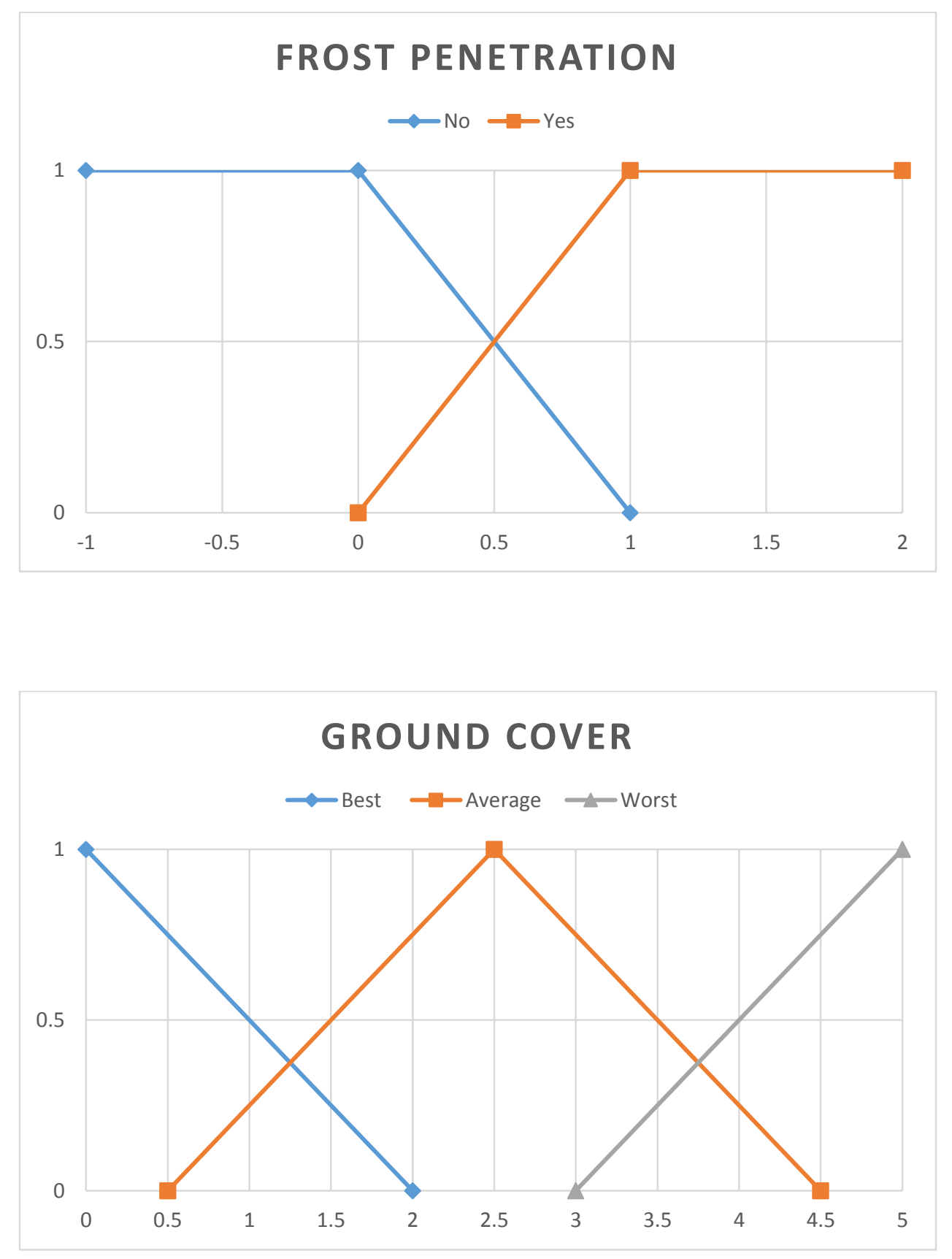

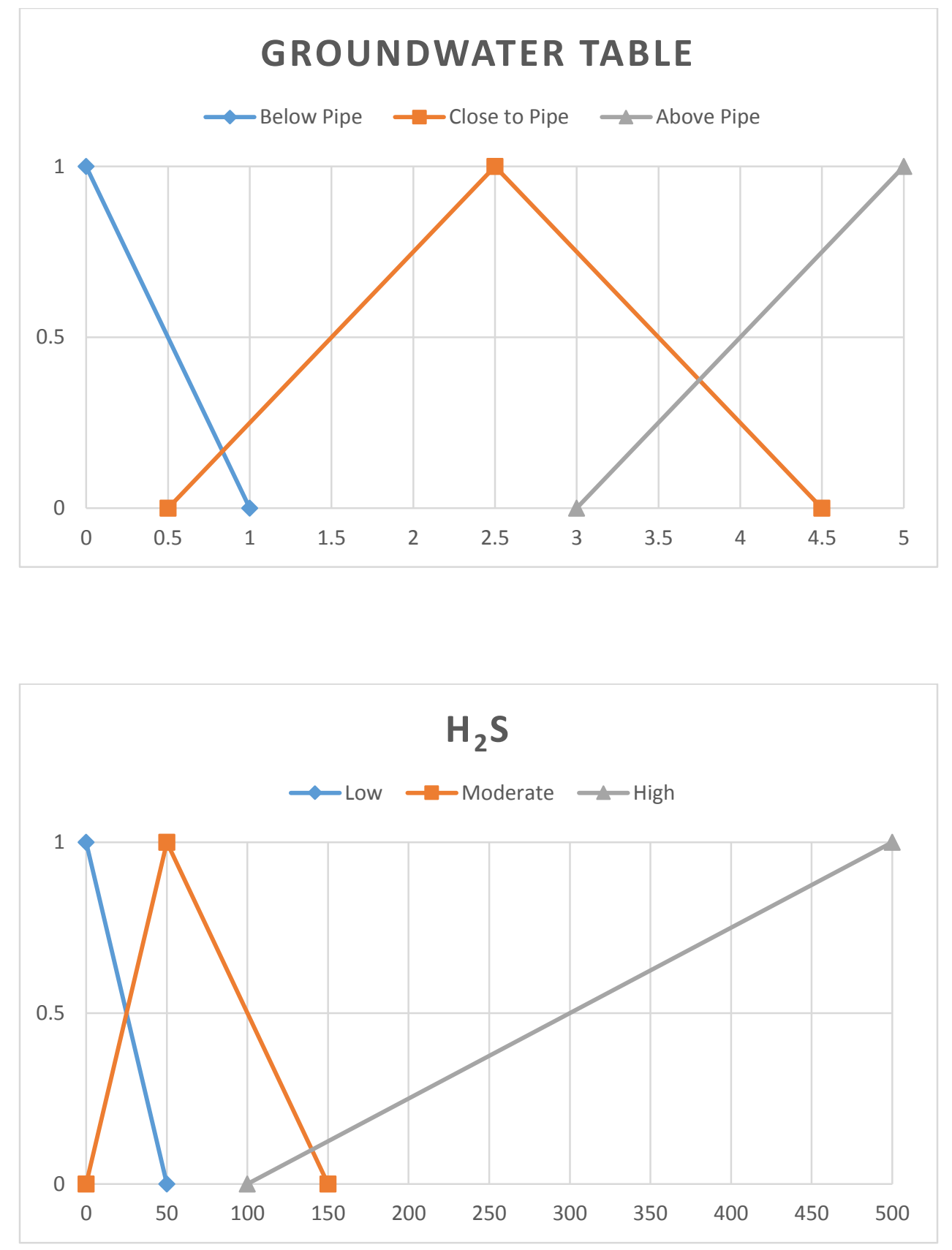

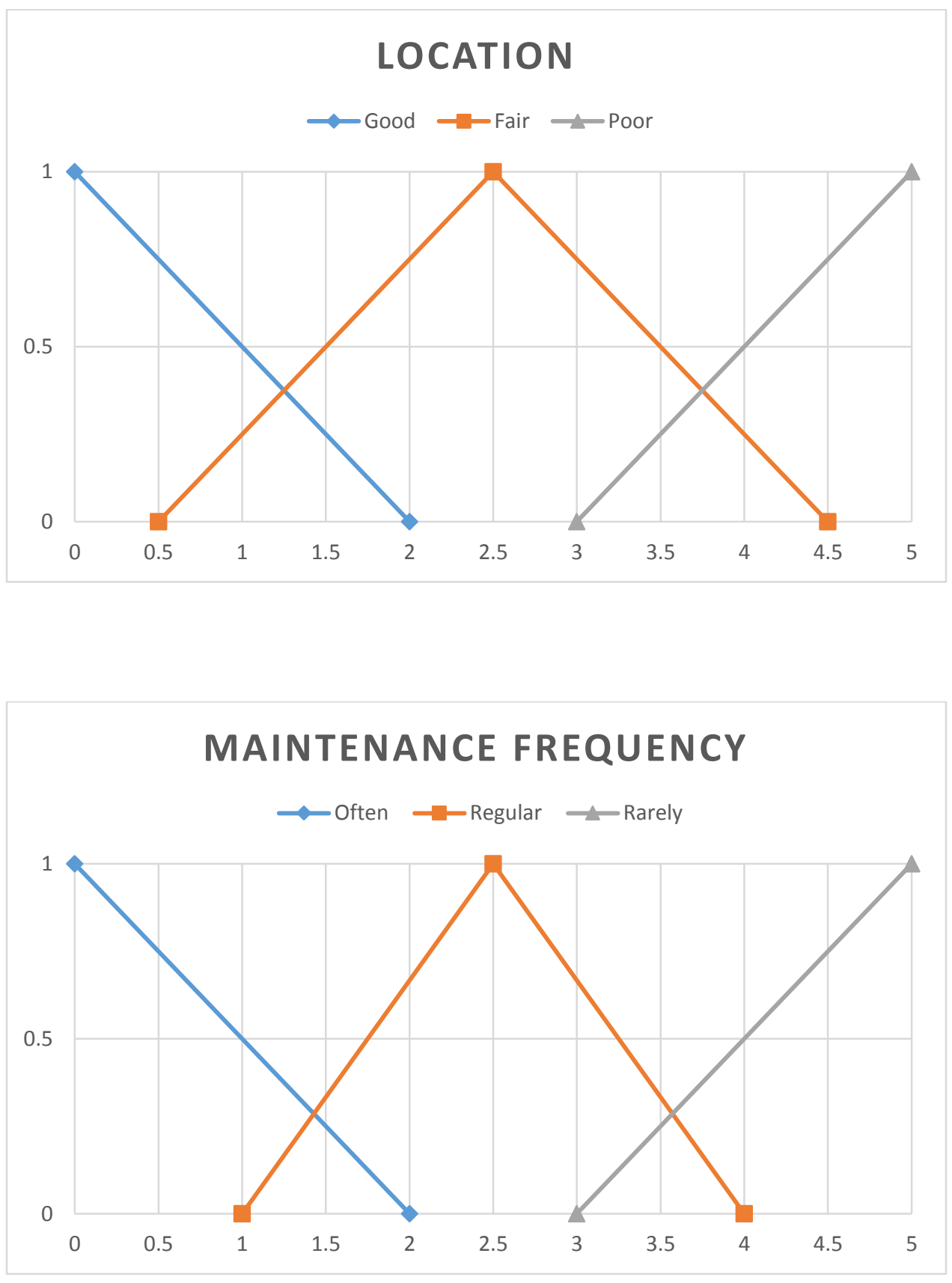

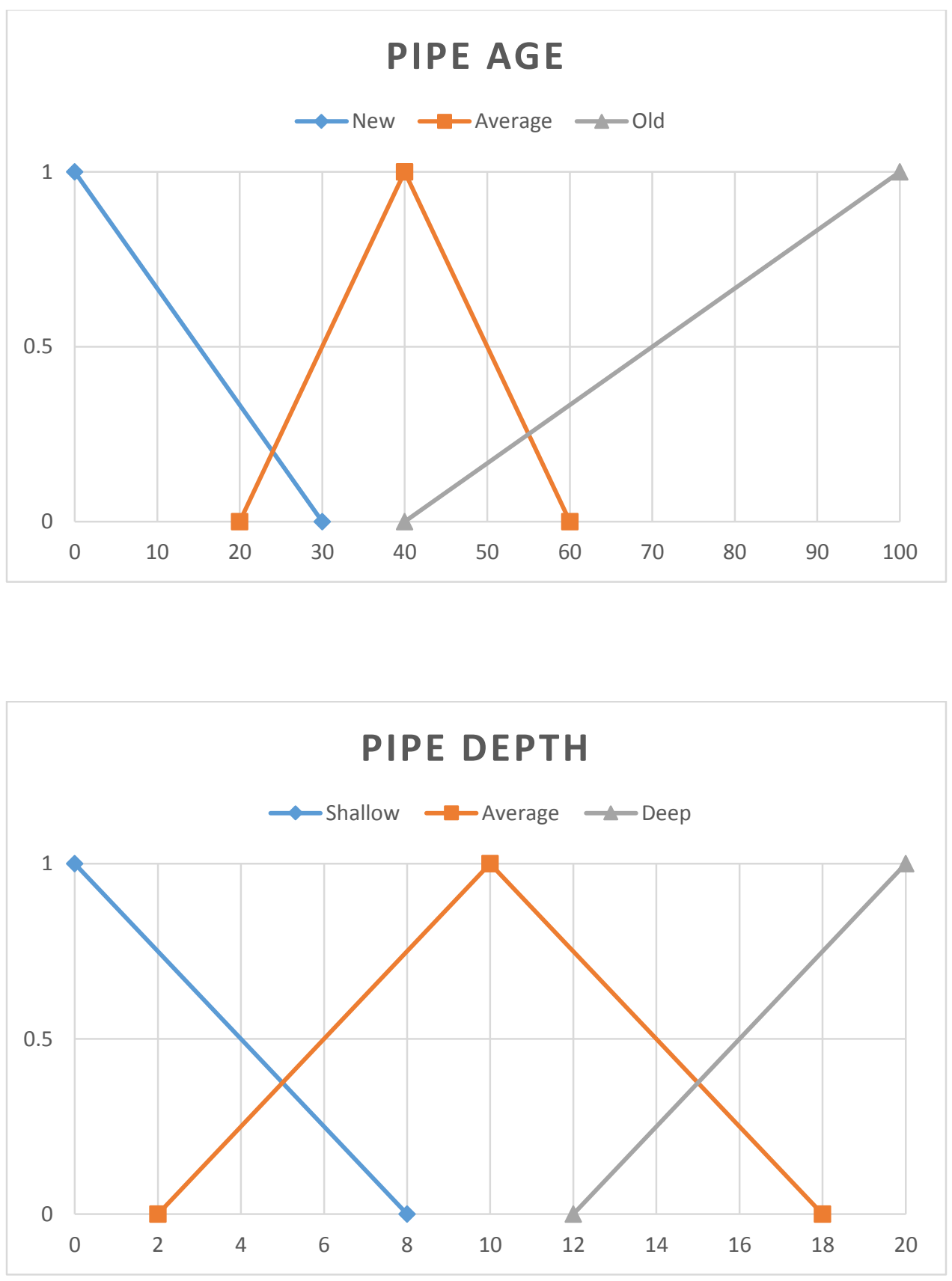

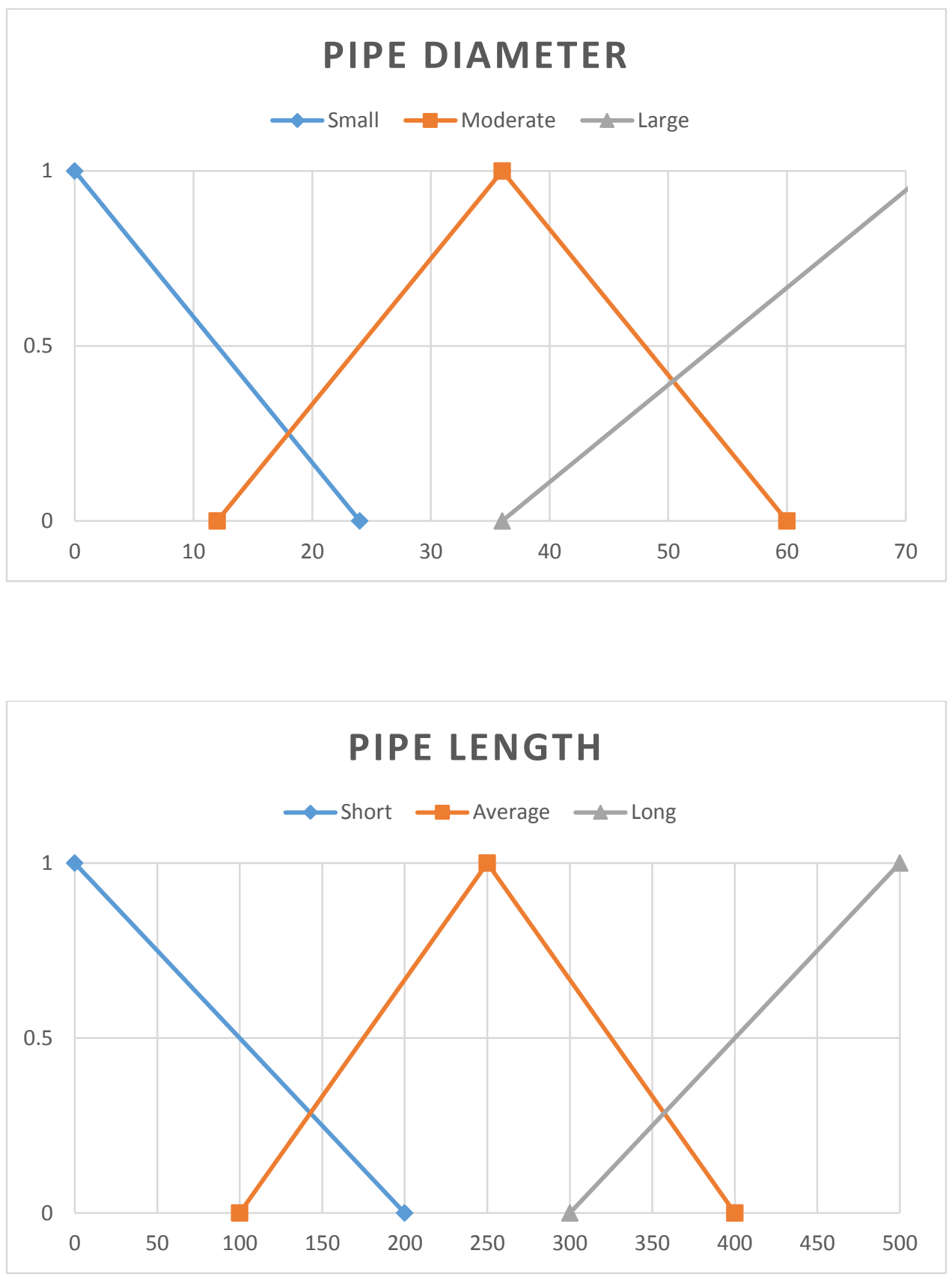

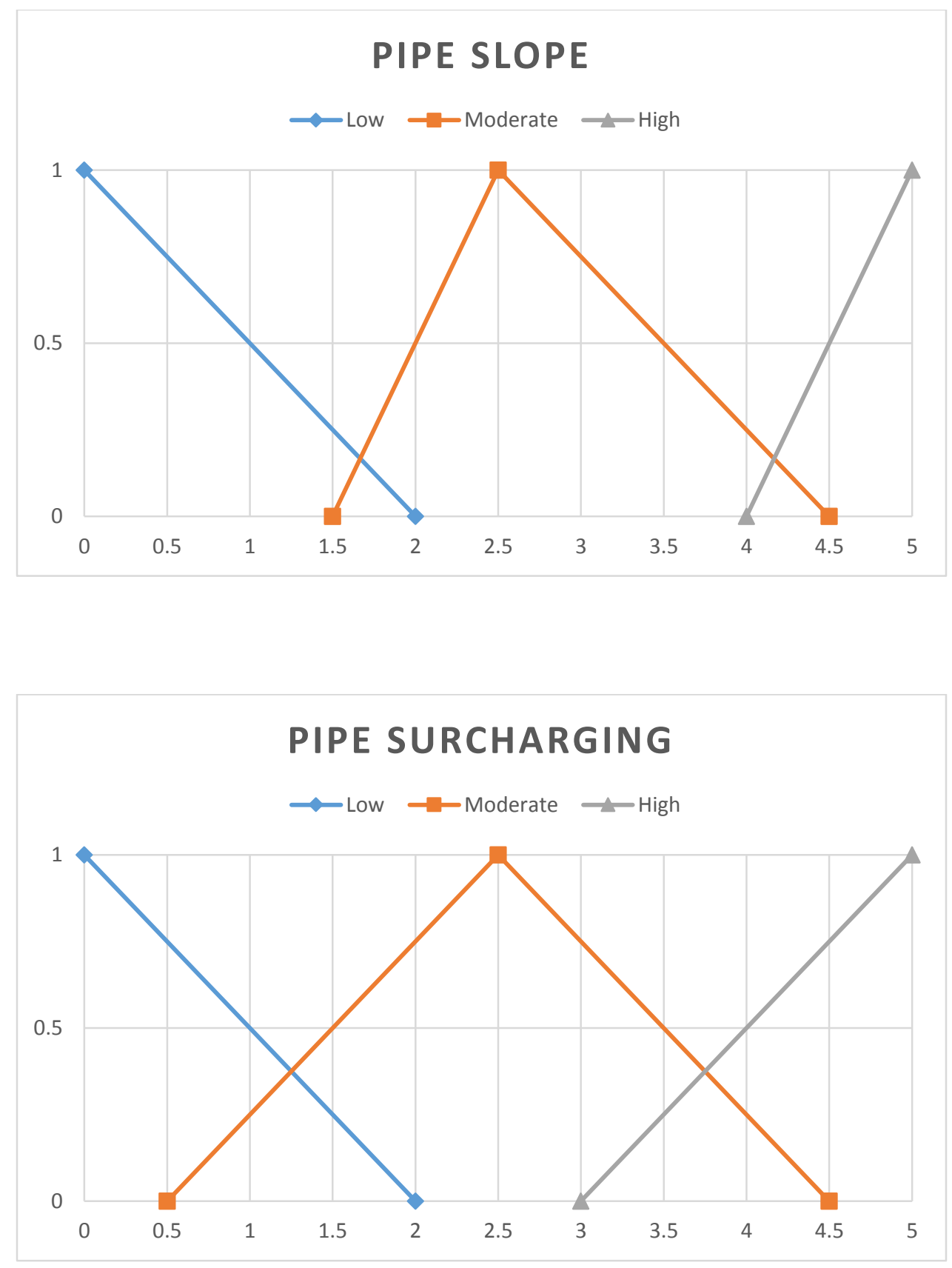

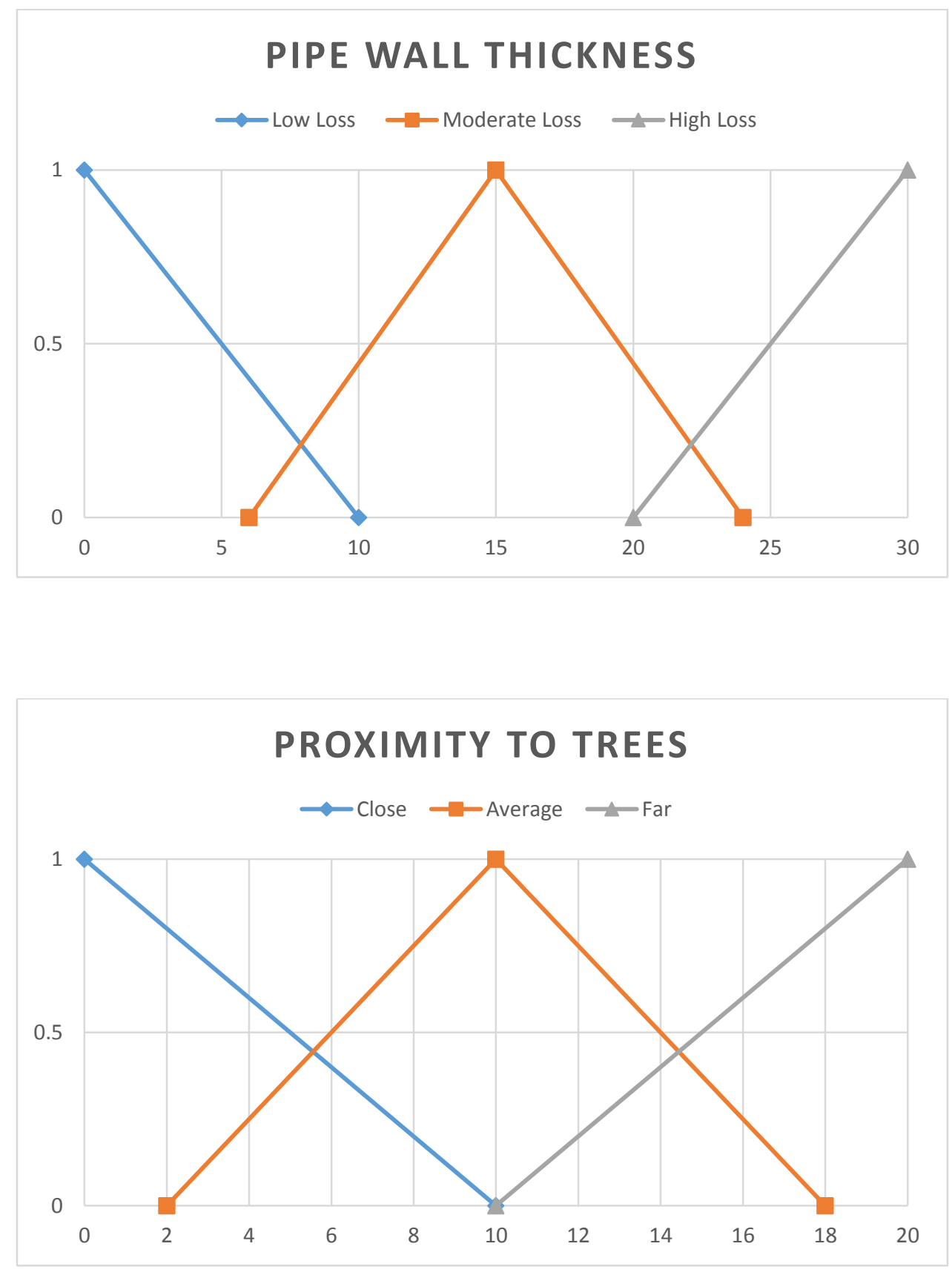

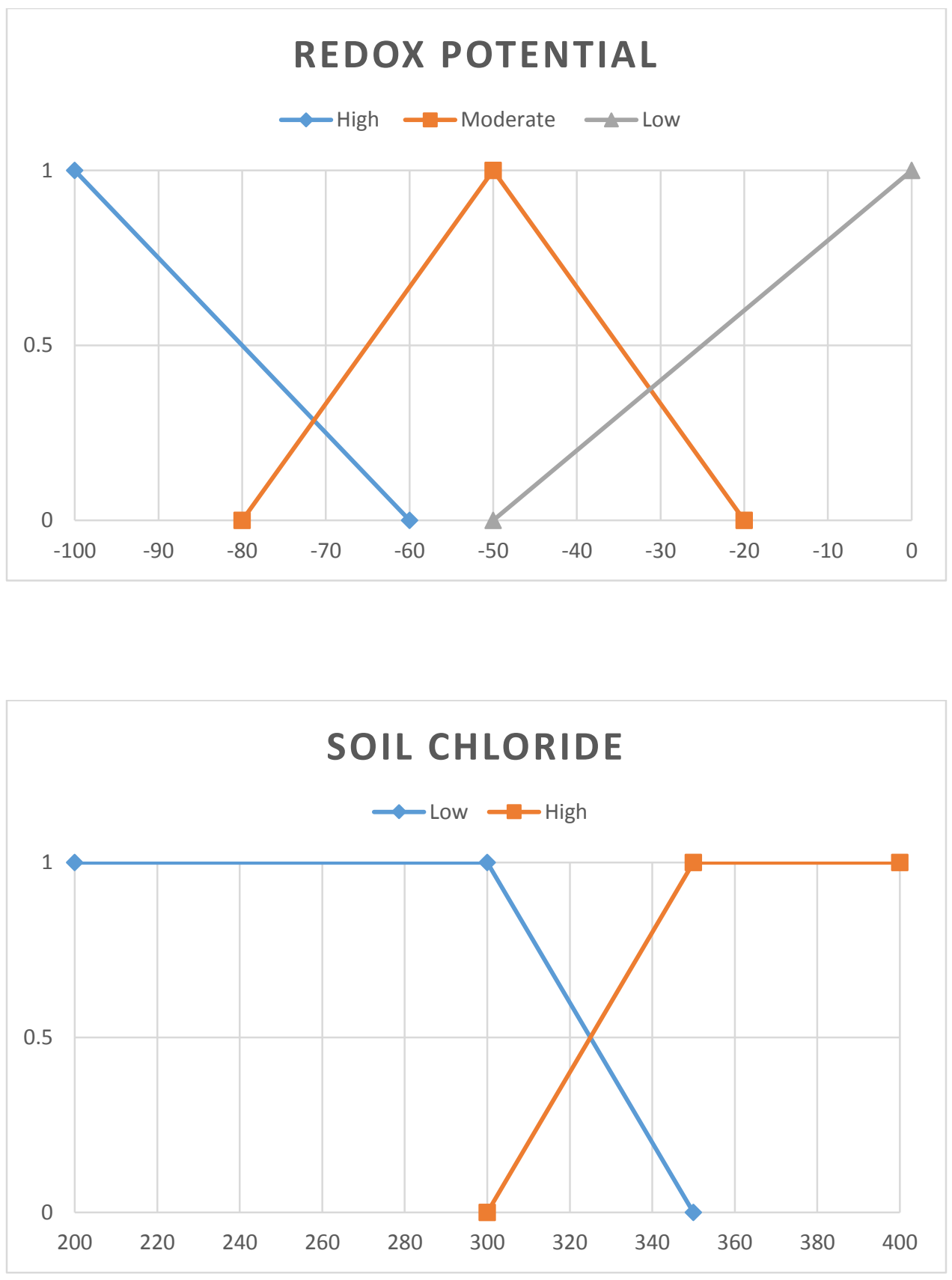

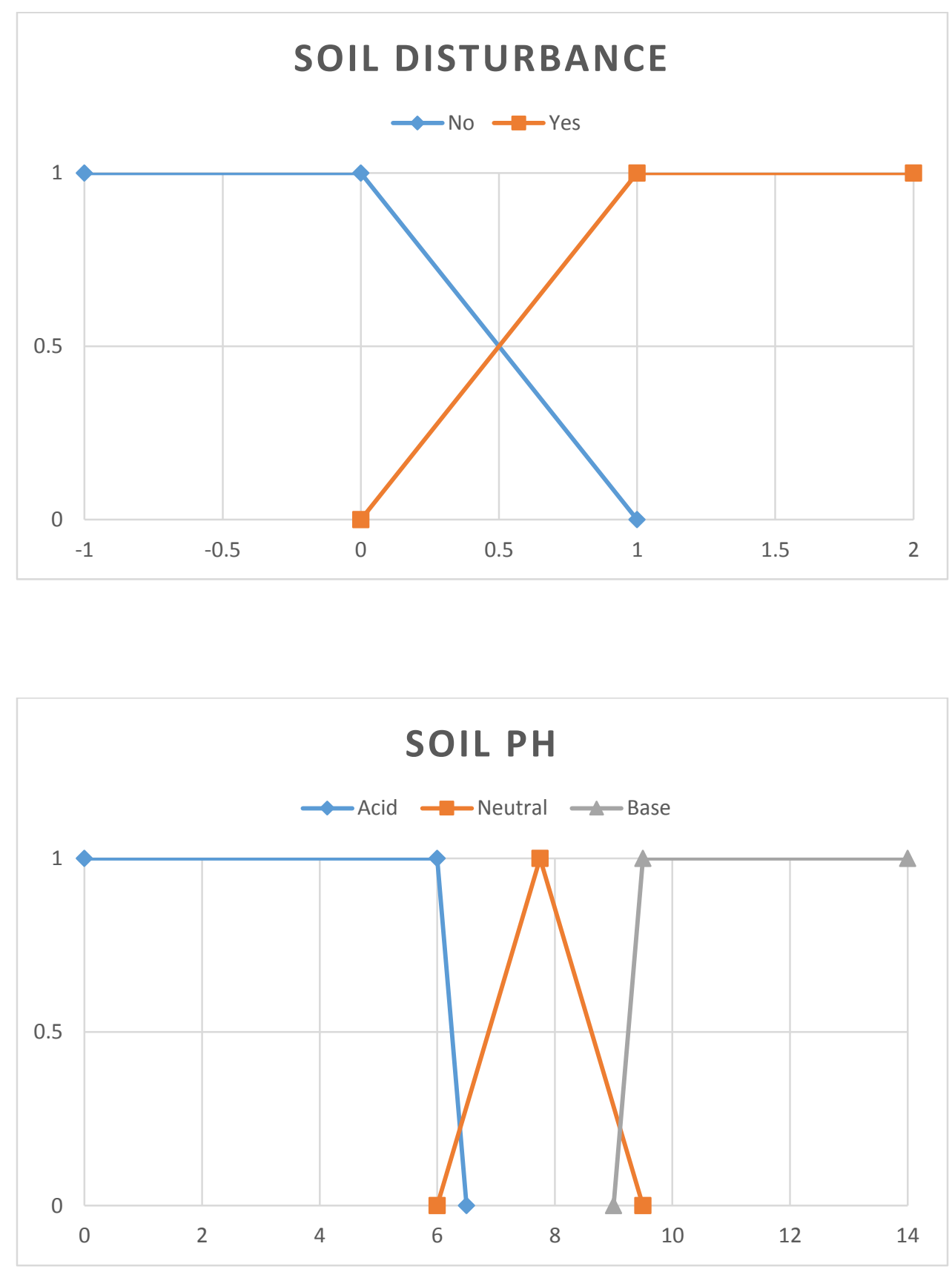

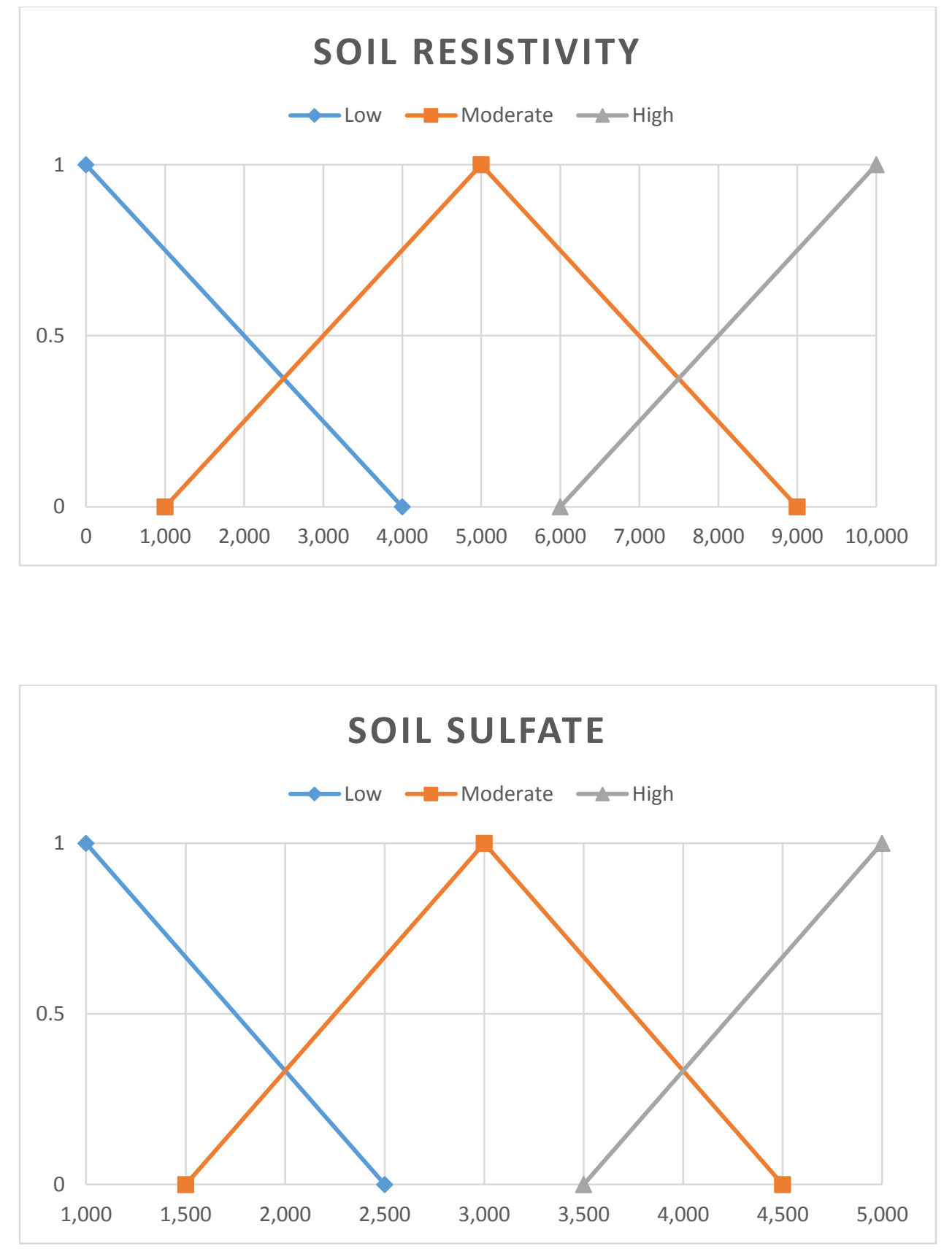

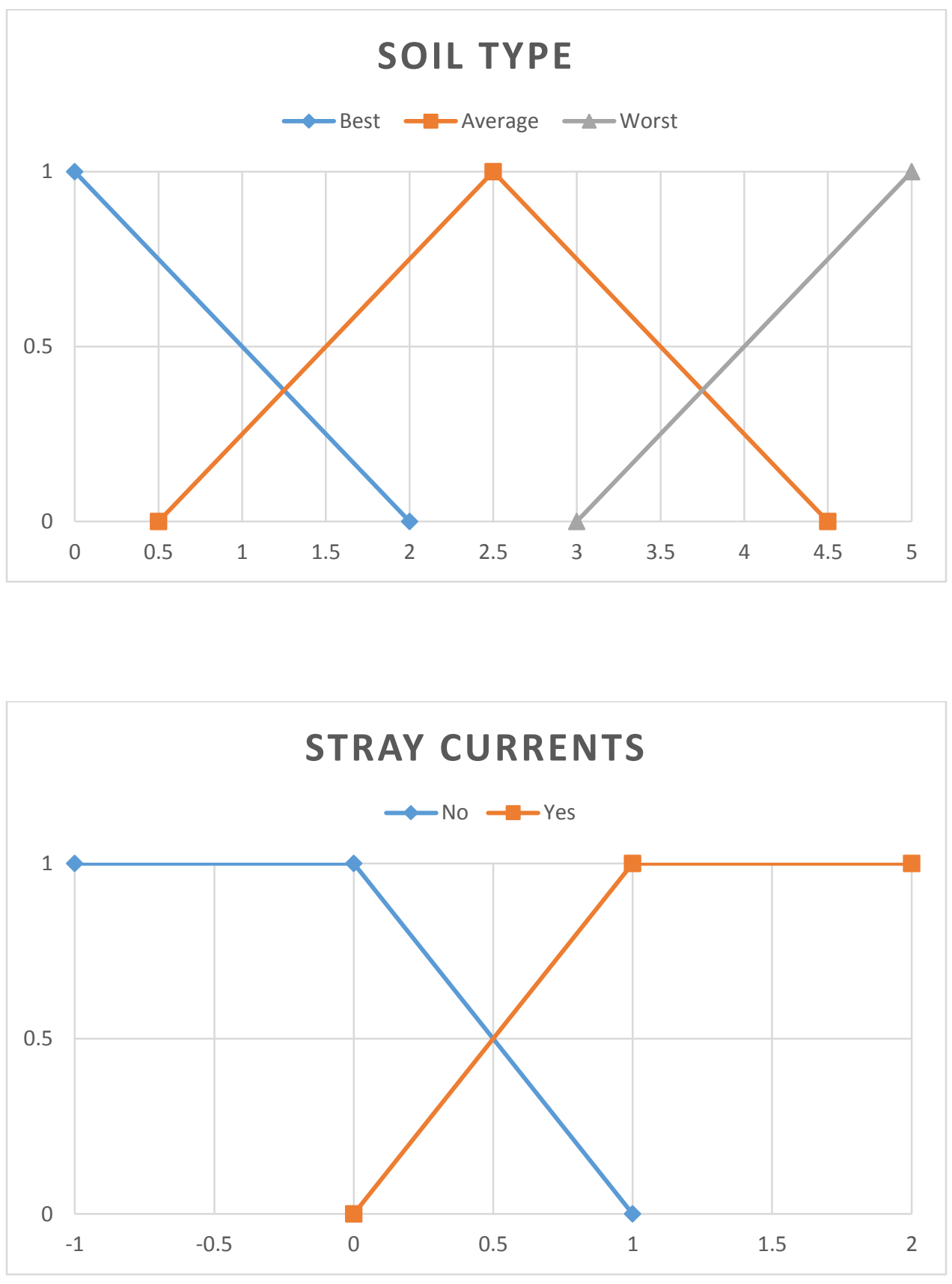

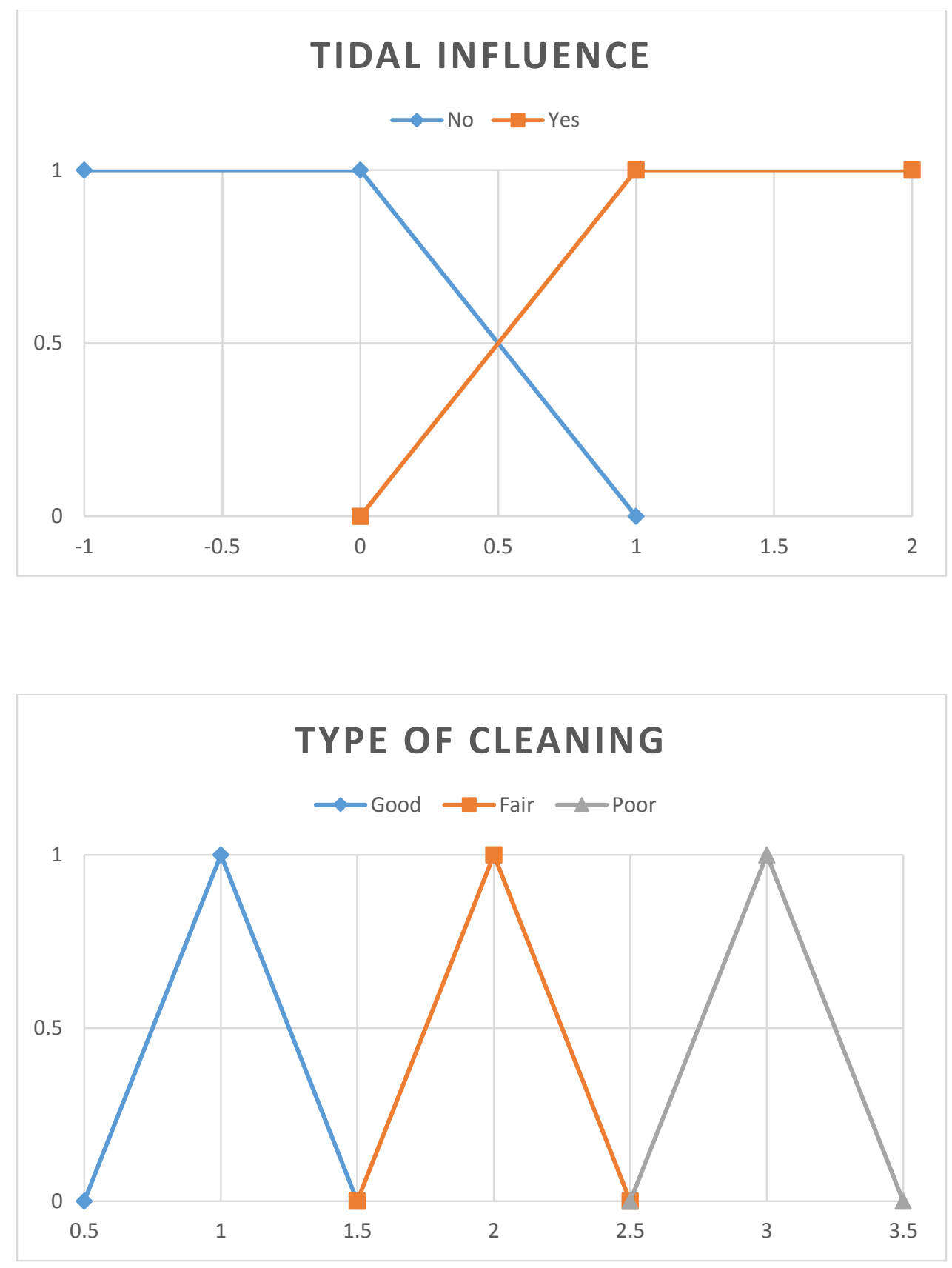

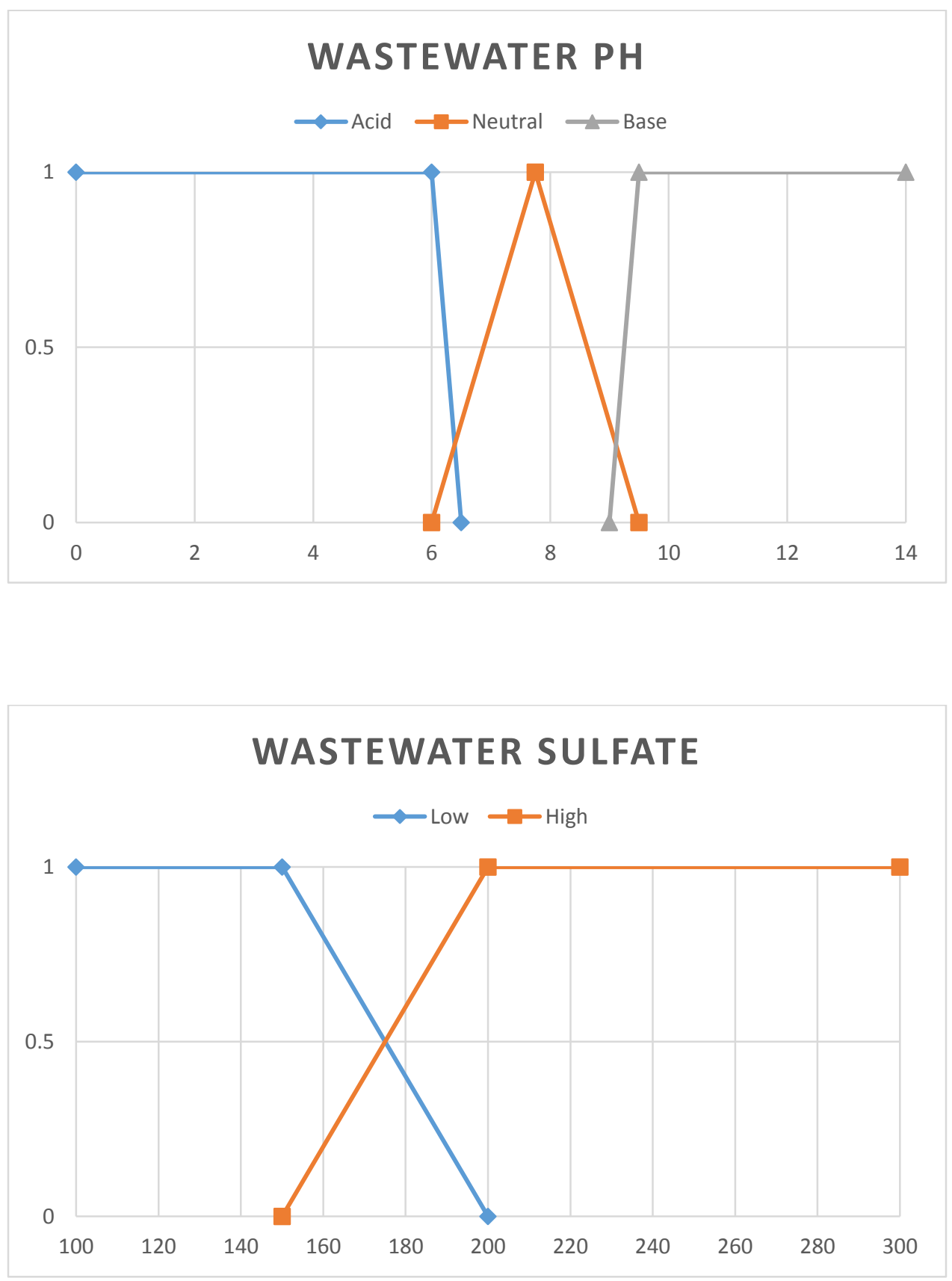

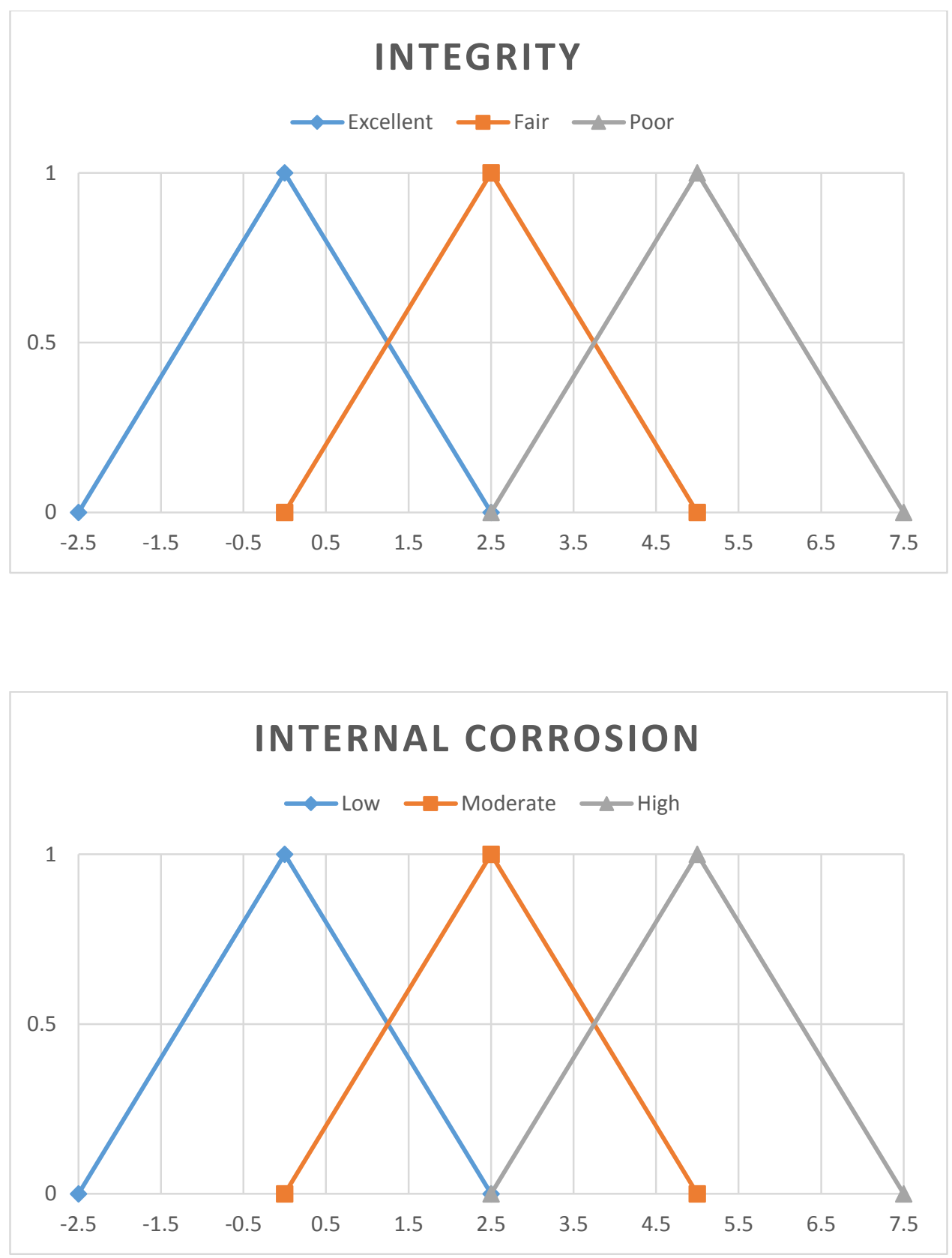

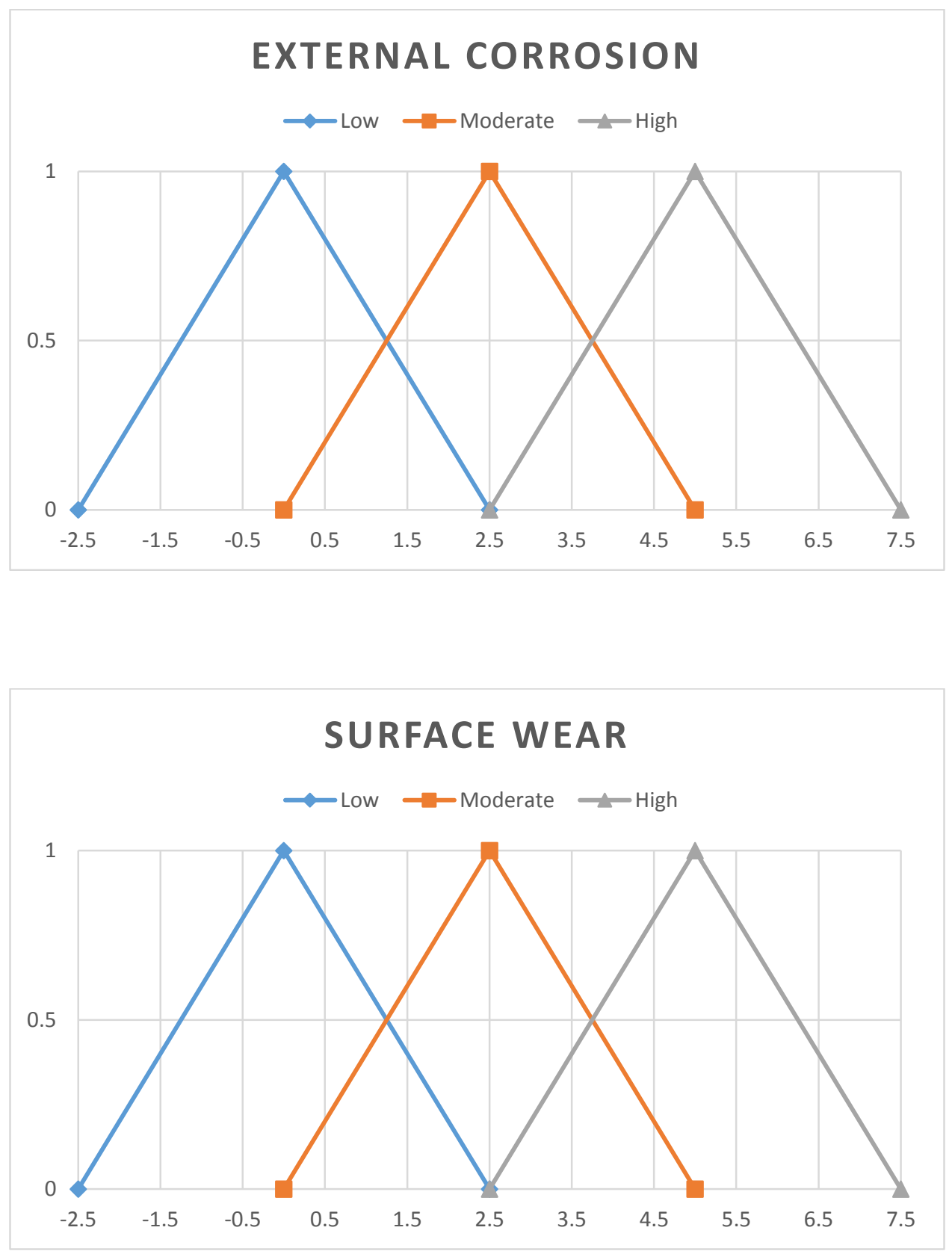

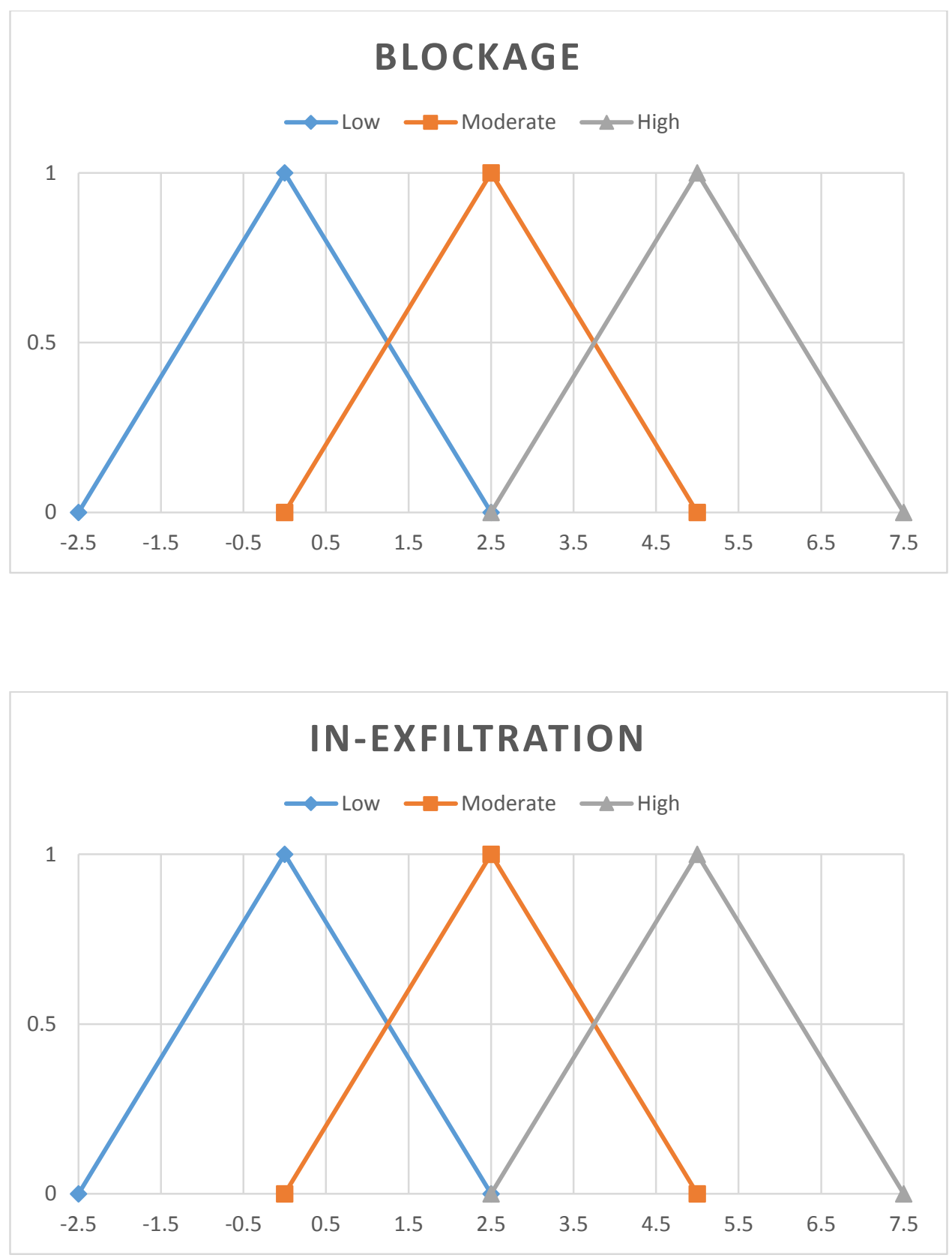

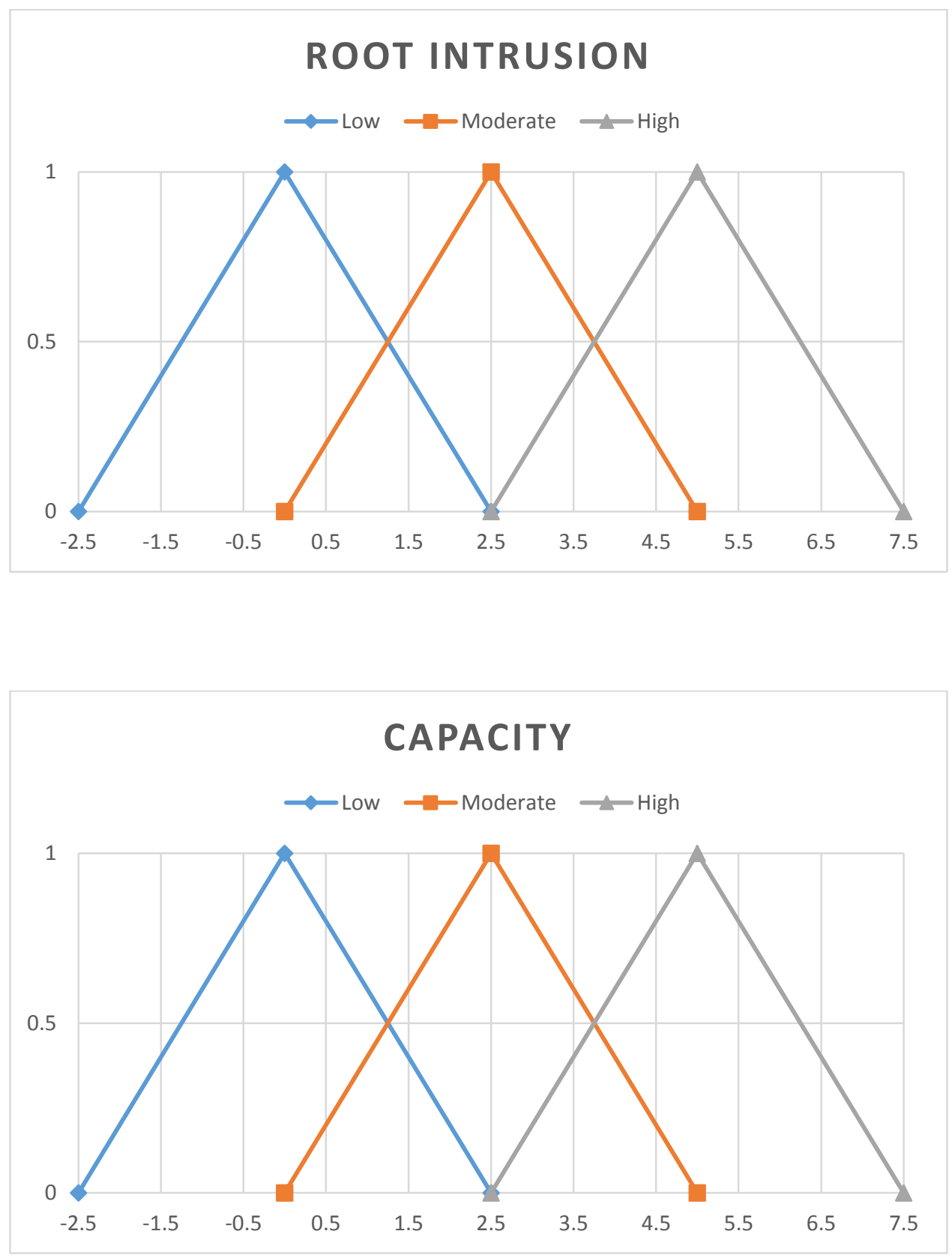
APPENDIX E

\section{IF-THEN RULES}




\section{Rule: Integrity Module}

1. If (condition is good) then (integrity is excellent) (1)

2. If (condition is fair) then (integrity is fair) (1)

3. If (condition is poor) then (integrity is poor) (1)

4. If (condition is fair) and (bedding condition is poor) then (integrity is poor) (0.5)

5. If (location is poor) and (pipe depth is shallow) then (integrity is poor) (0.5)

6. If (soil type is worst) and (groundwater table is above pipe) then (integrity is poor) $(0.3)$

7. If (ground cover is worst) then (integrity is poor) (0.3)

8. If (pipe surcharging is high) then (integrity is poor) (0.2)

9. If (pipe age is old) then (integrity is poor) (0.7)

10. If (flooding is yes) then (integrity is poor) (0.3)

11. If (soil disturbance is Yes) then (integrity is poor) (0.3)

12. If (frost penetration is yes) then (integrity is poor) (0.3)

13. If (pipe age is average) then (integrity is fair) (0.4)

14. If (pipe depth is shallow) then (integrity is poor) (0.3)

\section{Rule: Internal Corrosion Module}

1. If (condition is good) then (internal corrosion is low) (1)

2. If (condition is fair) then (internal corrosion is moderate) (1)

3. If (condition is poor) then (internal corrosion is high) (1)

4. If (condition is fair) and (pipe age is old) then (internal corrosion is high) (0.5)

5. If (pipe slope is low) or (flow velocity is low) then (internal corrosion is high) (0.1)

6. If (condition is fair) and (wasterwater ph is acid) then (internal corrosion is high) $(0.3)$

7. If (condition is fair) and (wasterwater ph is base) then (internal corrosion is high) $(0.3)$

8. If (condition is fair) and (maintenance frequency is rarely) then (internal corrosion is high) (0.5)

9. If ( $\mathrm{H} 2 \mathrm{~S}$ is high) then (internal corrosion is high) (0.5)

10. If (wastewater sulfate is high) then (internal corrosion is high) (0.3)

11. If $\left(\mathrm{H}_{2} \mathrm{~S}\right.$ is moderate) and (flow depth/diameter is Empty) then (internal corrosion is high) (0.3) 


\section{Rule: External Corrosion Module}

1. If (pipe age is old) and (soil resistivity is low) then (external corrosion is high) $(0.5)$

2. If (pipe age is average) and (soil resistivity is low) then (external corrosion is moderate) (0.3)

3. If (soil ph is base) then (external corrosion is high) (0.2)

4. If (soil ph is acid) then (external corrosion is high) (0.2)

5. If (groundwater table is close to pipe) then (external corrosion is high) $(0.3)$

6. If (stray currents is Yes) then (external corrosion is high) (0.5)

7. If (soil sulfate is high) then (external corrosion is high) (0.5)

8. If (soil chloride is high) then (external corrosion is high) (0.5)

9. If (redox potential is high) then (external corrosion is high) $(0.5)$

10. If (pipe wall thickness is low loss) then (external corrosion is low) (1)

11. If (pipe wall thickness is moderate loss) then (external corrosion is moderate) (1)

12. If (pipe wall thickness is high loss) then (external corrosion is high) (1)

13. If (soil resistivity is low) then (external corrosion is high) $(0.5)$

\section{Rule: Surface Wear Module}

1. If (condition is good) then (surface wear is low) (1)

2. If (condition is fair) then (surface wear is moderate) (1)

3. If (condition is poor) then (surface wear is high) (1)

4. If (condition is poor) and (flow velocity is high) then (surface wear is high) (0.5)

5. If (pipe age is old) then (surface wear is high) (0.3)

6. If (pipe age is old) and (pipe slope is high) then (surface wear is high) (0.2)

7. If (type of cleaning is poor) then (surface wear is high) (0.5)

8. If (pipe age is old) and (flow velocity is high) then (surface wear is high) (0.2) 


\section{Rule: Blockage Module}

1. If (condition is good) then (blockage is low) (1)

2. If (condition is fair) then (blockage is moderate) (1)

3. If (condition is poor) then (blockage is high) (1)

4. If (condition is fair) and (pipe age is old) then (blockage is high) (0.5)

5. If (pipe length is long) then (blockage is high) (0.3)

6. If (pipe slope is low) or (flow velocity is low) then (blockage is high) (0.2)

7. If (pipe diameter is small) then (blockage is moderate) (0.1)

8. If (flow depth/diameter is empty) and (maintenance frequency is rarely) then (blockage is high) $(0.3)$

9. If (density of connections is very dense) then (blockage is high) (0.3)

10. If (maintenance frequency is rarely) then (blockage is high) (0.3)

11. If (pipe age is old) and (maintenance frequency is regularly) then (blockage is moderate) (0.3)

12. If (pipe age is old) and (maintenance frequency is rarely) then (blockage is high) (0.5)

13. If (pipe age is old) and (pipe diameter is small) then (blockage is high) (0.2)

\section{Rule: Capacity Module}

1. If (condition is good) then (capacity is low) (1)

2. If (condition is fair) then (capacity is moderate) (1)

3. If (condition is poor) then (capacity is high) (1)

4. If (pipe slope is low) or (flow velocity is low) then (capacity is high) (0.2)

5. If (pipe surcharging is high) then (capacity is high) (0.5)

6. If (maintenance frequency is rarely) then (capacity is high) (0.5)

7. If (flooding is yes) or (tidal influence is yes) then (capacity is high) (0.5) 


\section{Rule: In-exfiltration Module}

1. If (condition is good) then (infiltration \& exfiltration is low) (1)

2. If (condition is fair) then (infiltration $\&$ exfiltration is moderate) (1)

3. If (condition is poor) then (infiltration \& exfiltration is high) (1)

4. If (condition is fair) and (pipe age is old) then (infiltration \& exfiltration is high) $(0.5)$

5. If (soil type is worst) then (infiltration \& exfiltration is high) (0.2)

6. If (pipe surcharging is high) then (infiltration \& exfiltration is high) (0.5)

7. If (groundwater table is above pipe) then (infiltration \& exfiltration is high) (0.3)

\section{Rule: Root Intrusion Module}

1. If (condition is good) then (root intrusion is low) (1)

2. If (condition is fair) then (root intrusion is moderate) (1)

3. If (condition is poor) then (root intrusion is high) (1)

4. If (condition is fair) and (pipe diameter is small) then (root intrusion is high) (0.3)

5. If (condition is poor) and (pipe diameter is small) then (root intrusion is high) (0.5)

6. If (condition is fair) and (proximity to tree is close) then (root intrusion is high) (0.5)

7. If (condition is fair) and (maintenance frequency is rarely) then (root intrusion is high) (0.3)

8. If (pipe age is old) and (maintenance frequency is rarely) then (root intrusion is high) $(0.3)$

9. If (condition is poor) and (proximity to trees is close) then (root intrusion is high) $(0.5)$

10. If (pipe age is old) and (maintenance frequency is regularly) then (root intrusion is moderate) (0.3)

11. If (pipe diameter is small) and (maintenance frequency is regular) then (root is moderate) (0.3) 


\title{
APPENDIX F
}

\author{
UTILITY DATA
}




\section{Overview of the Participating Utilities and Utility Data}

\section{Seattle Public Utilities Seattle, WA}

Seattle Public Utilities (SPU) owns and operates the wastewater collection system for the city of Seattle, comprising 1,491 miles of combined sewer and sanitary pipelines. The wastewater from the city is treated at King County sewage treatment plants. The city's wastewater system services 570,000 people (Ross \& Associates Environmental Consulting, 2005). SPU's management system includes, a twenty-year comprehensive plan providing longterm direction setting, a three-year strategic plan setting the objectives and targets in line with the comprehensive plan; and specific management systems supporting the objectives and targets of the strategic plan (Ross \& Associates Environmental Consulting, 2005). The system plan set in 2006, has focused on describing all of SPU's existing wastewater policies and identifies

- areas where additional policies need to be developed;

- levels of customer service to minimize sewer backups, street flooding in combined sewer areas, control of combined sewer overflows, and emergency responses;

- presents strategies and an implementation plan to meet the established customer service levels; and

- establishes a financial program to fund the programs and activities in the plan (Brown and Caldwell, 2006).

SPU gave shape-files, geo-guide, CCTV excel files to the research group. Altogether there were 14 shape-files along with a geo-guide which actually is like a metadata file describing the attributes mentioned in the shape files. These consist mainly of the main pipelines, laterals, manholes, catch basins, and drainage basins along with transportation and terrain. A brief description of the main files given by SPU is given below.

Mainline shape files consists of all the sewer mains in Seattle city limits which are spatially mapped in the projected coordinate system NAD_1983_StatePlane_Washington_South. The sewer main attribute table consists of various pipe parameters such as Pipe ID, shape, diameter, installation year, material, length, elevation of the upstream and downstream nodes Although a few columns were left blank, enough information was collected.

Manhole shape files consists of Manholes in Seattle city limits which are spatially mapped in the projected coordinate system NAD_1983_StatePlane_Washington_South. The shape files consist of various manhole parameters such as facility ID, topographical location, depth of the manhole, diameter, and the geographical coordinates. Some inner details like case type, probable flow are also given. 
Drainage basin shape files are a collection of parameters of the drainage basins in Seattle city limits like area, perimeter, and outfall numbers. The closest water bodies are also listed along with the catalog IDs of the basins. Altogether, there are 159 drainage basins.

\section{Western Virginia Water Authority, Roanoke, VA}

The water and wastewater operations of the City of Roanoke and Roanoke County are officially consolidated as the Western Virginia Water Authority (WVWA). WVWA is responsible for operating the Roanoke Regional Water Pollution Control Plant, which treats 40 million gallons of wastewater a day from throughout the Roanoke Valley. CCTV, pole inspection, cleaning (jetting and rodding), smoke testing, and zoom camera are the condition assessment techniques regularly used by WVWA. The problems often encountered are cracks, roots, blockage (grease) sags, belling on the line, and collapses. Currently WVWA uses PACP as its rating system but there is a plan to move to an in-house rating system. The wastewater pipes are inspected and the data is updated to GIS on a regularly.

WVWA has given its database to the research group at Virginia Tech. Altogether, there were 15 geo-database tables which primarily consist of WVWA inspection and maintenance records, sewer main tables and manhole tables. There are approximately 35,000 pipe sections. The majority of the wastewater pipes are vitrified clay, concrete, and PVC. In the mainline table, there are many fields including pipe age, length, slope, depth, and. However, many cells are blank.

\section{Orange County Sanitation District, Orange County, CA}

The Orange County Sanitation District (OCSD), formed in 1946, collects, treats, disposes, and reclaims wastewater generated by 2.5 million people in central and northwestern Orange County. OCSD includes nine former revenue areas joined into a single service district, forming the third largest wastewater agency in the western United States (Ross \& Associates Environmental Consulting, 2005). OCSD operates 2 treatment plants and maintains 580 mile of wastewater pipes, and 16 pumping stations which 250 million gallons of wastewater flows through daily. One treatment plant is located in Fountain Valley and the other in Huntington Beach.

Ten Million gallons per day treated wastewater are reclaimed via microfiltration and reverse osmosis. The reclaimed water is used for landscape irrigation and for injection into the groundwater seawater intrusion barrier. Recently, in cooperation with the Orange County Water District, the Ground Water Replenishment System was started. Using advanced water treatment facilities, water will be purified through microfiltration, reverse osmosis, and ultraviolet disinfection to levels that far exceed drinking water standards. In addition OCSD has undertaken management systems initiatives in two main areas: Enterprise Asset Management program and the National Biosolids Partnership Environmental Management System. OCSD has also engaged in strategic planning activities and created the Unifying Strategies. 
OCSD gave shape files to the research group. Altogether there were 13 shape-files. These consist mainly of the main pipelines, manholes, land use along with soils and city limits. A brief description of the OCSD main files is given below. The sewer system in the city also has some force main pipes along with fittings and valves.

The sewer main shape file consists of all mains in Orange County which are spatially mapped in the projected coordinate system NAD_1983_StatePlane_California_VI. The sewer main attribute table consists of various pipe parameters such as Pipe ID, pipe shape, diameter, material, length, and elevation of the upstream and downstream nodes. Also, the slope of the pipe was calculated and added as an attribute. Although a few columns were blank, enough information was collected for around 9000 pipes.

The manhole shape file consists of Manholes in Orange County which are spatially mapped in the projected coordinate system NAD_1983_StatePlane_California_VI. The shape file consists of various manhole parameters such as facility ID, installation date, depth, diameter and the geographical coordinates. The document numbers and project ID's are also noted. Although a few columns were blank, enough information was collected for around 8000 manholes.

\section{Pittsburgh Water and Sewer Authority, PA}

The Pittsburgh Water and Sewer Authority (PWSA) was founded in 1984. In 1995, the City of Pittsburgh's Water Department became a part of PWSA. PWSA serves approximately 250,000 consumers throughout the City of Pittsburgh (PWSA, n.d.)

Recently PWSA has proposed the CSO program which aims to identify cost-effective CSO control alternatives that, when fully implemented, protect water quality (PWSA, 2003). The development of this program required monitoring and sampling plans that proposed data collection and characterization activities for a Long Term Control Plan (LTCP). The LTCP is the characterization of the combined sewer system operation and the assessment of CSO impacts on river and stream water quality during wet weather events. The Monitoring and Sampling Plan presents the proposed data collection and characterization activities to be undertaken.

The Pittsburg Water and Sewer Authority (PWSA) and their consultant gave shape-files to the research group. In total, there were eight shape-files consisting primarily of the main pipelines, manholes, junctions, and diameter changes. A brief description of the main files given by PWSA is described below. One characteristic of these shape files is that the same attributes are listed for all even though they are unrelated. Such attributes are either given a null value or left empty.

The sewer main shape file consists of all the sewer mains within the Pittsburgh city limits which are spatially mapped in an undefined, projected coordinate system. The sewer main attribute table consists of various pipe parameters such as Pipe ID, shape, diameter, material, 
length, installation year, and elevation of the upstream and downstream nodes. Although a few columns were blank, enough information was collected for approximately 44,000 pipes.

The manhole shape files contain information on all the manholes in Pittsburgh city limits which are spatially mapped in an undefined projected coordinate system. The shape file consists of various manhole parameters such as facility ID, depth of the manhole, diameter and the geographical coordinates. Although a few columns were blank, enough information was collected for around 30,000 manholes.

\section{Massachusetts Water Resources Authority, Boston, MA}

Massachusetts Water Resources Authority (MWRA) is a public authority founded in 1984. It provides water and sewer services to 2.5 million people and more than 5,500 large industrial users in 61 metropolitan Boston communities.

\section{Statistics}

- 2.5 million people served

- 890,000 households served

- 5,500 businesses served

- 350 million gallons per day of sewage treated (average)

- 43 sewerage communities

Majority of MWRA wastewater pipes are brick pipes which account for $54 \%$ of the entire system. VC vitrified clay $16 \%$ and reinforced concrete $12 \%$. MWRA wastewater pipeline dated back hundreds years. $33 \%$ of pipes are in a range of 101-125 years old.

The Massachusetts Water Resources Authority (MWRA) and has given its geo-data table to the research group at Virginia Tech. We have analyzed data received from MWRA and evaluated each pipe section (manhole to manhole) using performance models (weighted factor and fuzzy inference system). The table mainly consist of MWRA's inspection and maintenance records, and general sewer main data. Parameters that are in the table include pipe age, pipe diameter, pipe depth, pipe slope, pipe length, pipe material, location, and pipe condition, for example.

\section{VPI Sanitary Authority and Town of Blacksburg, Blacksburg, VA}

Blacksburg's Wastewater system is operated by Blacksburg VPI Sanitation Authority Inc. and the Town of Blacksburg. VPI Sanitary Authority Founded in 1962, VPI Sanitation Authority is a non-profit organization resulted from a collaborative effort between the Town of Blacksburg and Virginia Tech. The authority owns and operates one treatment plant at Stroubles Creek, and about 15 miles of sewer main with 14 employees. All sanitary and industrial 
wastewater treated by the plant comes from three customers which are Town of Blacksburg, Virginia Tech and part of Montgomery County. Proportions of the wastewater collected from each customer are calculated based on their tap water usage. On average, $22 \%, 75 \%$, and $3 \%$ of wastewater traveling to the plant is from Virginia Tech, Town of Blacksburg and Montgomery County, respectively.

The Town of Blacksburg (ToB) owns and operates 21 pumping stations and about 145 miles of sanitary sewer. The Town's system is divided into 17 basins called sewer sheds. Each of these basins is further divided into sub-sewer sheds.

The Town of Blacksburg Utility gave shape-files to the research. Altogether there were two shape-files and one geo-database tables. The shape-files consist of the main pipelines, manholes and a sewer shed geo-database table. A brief description of the main files is given below.

\section{Mainlines}

The sewer main shape file consists of all the sewer mains in Town of Blacksburg limits which are spatially mapped in the projected coordinate system NAD_1983_StatePlane_Virginia_South. The sewer main attribute table consists of various pipe parameters such as Pipe ID, diameter, material, section length, Joint Type, Street Address, connected manhole, manhole condition, and pipe condition. Also, the length of the pipe was calculated and added as an attribute. Although a few columns were left blank, enough information was collected for around 4700 pipes. Screenshot of Blacksburg's GIS is shown in figure F-2.

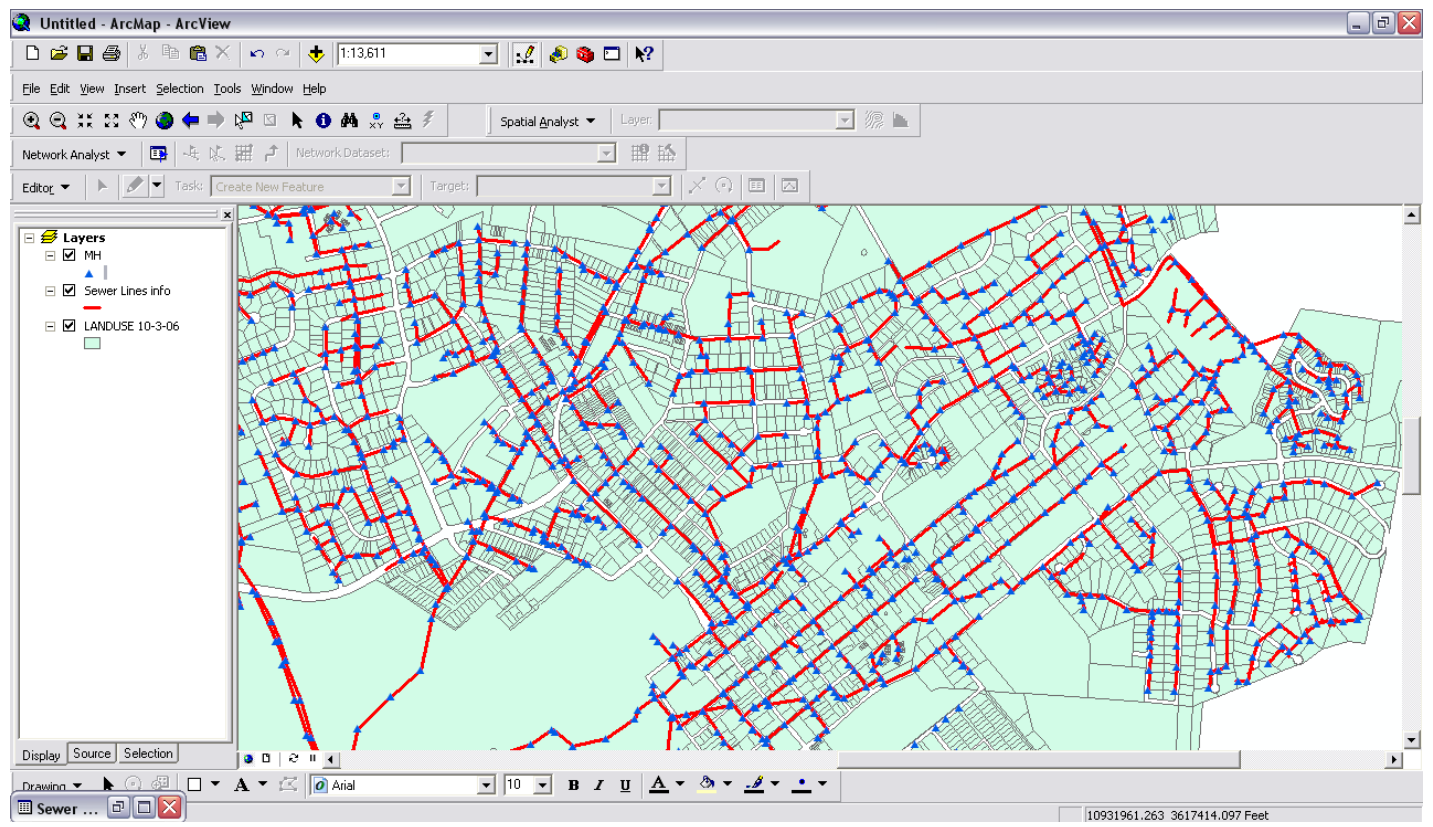

Figure F-2. Town of Blacksburg's GIS Data. 


\section{Manholes}

This consists of Manholes in town of Blacksburg limits which are spatially mapped in the projected coordinate system NAD_1983_StatePlane_Virginia_South. Except for the manholes condition presented in mainline shape-files, there is limited information in the manhole shapefile.

\section{Atlanta Public Utilities, GA}

There are approximately 40,000 manholes and 1,900 miles of sewer main and laterals with in right-of-way and easements of the city of Atlanta wastewater collection and transmission systems. The combined sewers are estimated to be $85 \%$ of the system and the rest are separate sanitary sewers. Cities of Hapeville, College Park and East Point, and DeKalb, Clayton, and Fulton counties are six other entitles that have a wastewater treatment contract with the city. The city of Atlanta generates $55 \%$ of sewage flows and the wholesale agencies have 45 percent. The total population that benefit from the system is 1.6 million (Hutchinson, 2007). In 1999, Atlanta entered into the First Amended Consent Decree (FACD) with the EPA. The FACD requires Atlanta to implement many of the programs associated with EPA's widely discussed wastewater collection system management initiative for capacity, management, operation and maintenance known as CMOM.

\section{Utility Data}

The Atlanta Public Utilities Board (APUB) has given shape-files, geo-database tables, and work management tables stored in Hansen ${ }^{\circledR}$. Altogether there were 14 shape-files, 29 geodatabase tables and around 1,200 Hansen ${ }^{\circledR}$ file tables. The shape-files consist mainly of the main pipelines, laterals, sewer facilities consisting of Manholes, rehab work related files along with transportation, soils, catch basins and city limits. A brief description of the main files given by APUB follows.

\section{Sewer Main}

The sewer main shape file consists of all the sewer mains in Atlanta city limits which are spatially mapped in the projected coordinate system NAD_1983_StatePlane_Georgia_West. The sewer main attribute table consists of various pipe parameters such as Pipe ID, pipe mailing address (street name, street number), pipe shape, diameter, material, length, and elevation of the upstream and downstream nodes. Also, the slope of the pipe was calculated and added as an attribute. The surface cover and geographical location of each pipe is given along with the sewershed and Basin references. Although a few columns were left blank, information was collected for about 40,000 pipes.

\section{Sewer Facility}

Sewer Facility consists of Manholes in Atlanta city limits which are spatially mapped in the projected coordinate system NAD_1983_StatePlane_Georgia_West. The shape file consists 
of various manhole parameters such as facility ID, facility mailing address (as in the street name, street no), topographical location, depth of the manhole, diameter and the geographical coordinates. Some inner details like cover type, cover diameter, wall type are also given along with sewershed and basin references. Although a few columns were left blank, information was collected for approximately 37,000 manholes.

\section{SSES Tables}

The Sewer System Evaluation Survey (SSES) tables are geo-database tables which can be used to store general information that does not need to be spatially mapped. The SSES tables given by APUB have all the codes used by the utility officials. In general, location type, manhole type, and street type codes are specified. Pipe condition data, defects, type of leak, inspection, and inventory data are also stored. However, all of these tables contain a lot of null valued columns which might require some metadata to explain the purpose of the attributes.

\section{Hansen Tables}

Around 1,200 Hansen tables were provided by APUB most of which are empty. Hansen is a work order management system used by the Atlanta utility officials. These tables mainly consist of the work orders issued by the officials. Some tables also consist of codes and other information which could not be understood. In this regard, the APUB was contacted for further detailed information.

\section{Cobb County Water System, GA}

The framework of the Cobb County's wastewater collection system was established in early 1970s as part of a comprehensive capital improvement program (CCWS, n.d.). Currently Cobb County Water System (CCWS) operates and maintains 2500 miles of sewer line and four water reclamation facilities. CCWS utilizes CCTV and external inspection as their main tools to ensure that the system is adequately maintained to meet the operating requirements. Problems often encountered include cracks, holes, broken pipes, offset joints, root intrusions, grease, and sewer taps protruding into sewer line. The staff is developing a wastewater asset database in which will be a major improvement to the data management operations. CCWS maintains the GIS system consisting of a large number of pipes, which has been updated regularly; however the parameters currently available are still limited. There are numerous efforts to populate the GIS including coordinating the manholes, and tracing the initial information about the pipes through material and year of installation.

CCWS is in an early stage of developing its wastewater pipe database so unfortunately we were provided with the data including only three parameters that can be used in this pilot study. 


\section{Aurora Water, Aurora, CO}

Aurora Water owned and operates water treatment plants and reservoirs providing drinking water to more than 310,000 people in Aurora, Colorado. Comprised of two enterprise funds, water, and wastewater, Aurora water is supported from tap fees, use fees and development fees.

AW provided its database to us. Altogether there was one geo-database table which is the sewer main table. There are approximately 28,000 pipe sections. The majority of the wastewater pipes are vitrified clay, concrete, and PVC pipes. In the geo database table, there are many fields including pipe age, pipe length, pipe slope, and pipe status, and some referencing ID for example.

\section{Anchorage Water and Wastewater Utility, AK}

AWWU operates five treatment facilities, 1,500 miles of pipe, and over 325,000 square feet of additional structures which provide water and collect wastewater to/from the municipality of Anchorage, including the three main regions of Northern Communities, Anchorage Bowl, and Girdwood. The water utility has a service area of 130.4 square miles and serves an estimated 236,749 people through 55,000 customer accounts. The sewer service area covers the municipality of Anchorage with a surface area of 1,850.9 square miles, and serves 249,613 people through 56,000 customer accounts. The five physical treatment facilities are Eklutna Water Treatment Facility (EWTF), Ship Creek Water Treatment Facility (SCWTF), Asplund Wastewater Treatment Facility (AWWTF), Eagle River Wastewater Treatment, Facility (ERWWTF), and Girdwood Wastewater Treatment Facility (GWWTF).

There were geo-database tables which mainly are AWWU inspection and maintenance records, sewer gravity mains, force mains and manhole and pump tables. There are approximately 20,000 pipe sections. The majority of the wastewater pipes are ductile iron, vitrified clay, asbestos-cement pipes. In the sewer main table, there are many fields including installation date, pipe length, pipe slope, pipe depth, and pipe material, pipe.

\section{Washington Suburban Sanitary Commission, Washington, DC}

WSSC is the eighth largest water and wastewater utility in the nation, serving nearly 1.8 million residents and approximately 460,000 customer accounts in Prince George's and Montgomery counties over an area of nearly 1,000 square miles. WSSC is the $8^{\text {th }}$ largest water and wastewater facility in the U.S. WSSC operates and maintains eight water and wastewater plants, more than 5,500 miles of fresh water pipeline and nearly 5,400 miles of sewer pipeline.

The key GIS data collected by WSSC for their wastewater collection system is listed Manhole

- Manholes Installation Date 
- Symbol Code

- Life cycle Status

- Material

- Diameter

- Elevation

- Ground Type

Pipes

- Material

- Shape

- Length

- Diameter

- Manholes connected (ID)

- Pressure

- Cross-section Area

- Maximum Capacity

- Symbol Code

- Pipe ID

- Type of Pipe- Pressure/Gravity

- Slope

Altogether there were two geo-database tables which consist of WSSC inspection and condition tables. There are approximately 45,000 pipe sections. The majority of the wastewater pipes are vitrified clay and concrete pipes. In the inspection table, there are many fields including pipe length, inspection date, address, cleaning method, weather, and location for example, However many cells are blank. Condition table consists of defects recorded according PACP standard. There are approximately 400,000 defects. 


\section{APPENDIX G}

\section{Utility CaSe Studies}




\section{City A}

We analyzed data received from the City A and evaluated each pipe section (manhole to manhole) using performance models (weighted factor and fuzzy inference system). The table mainly consist of City A's inspection and maintenance records, and general sewer main data. Four parameters were used in this pilot study.

\section{Parameters}
a. Pipe length
b. Pipe Material
c. Location
d. Pipe condition (inspection records)

The parameters listed above were stored as attributes in mainline table. The pipe conditions are rated using PACP standard.

Figure G-1 shows a bar chart of the performance index calculated by PACP rating index, W-PIE and F-PIE. The x-axis is the performance index and y-axis is the number of pipes that fall within each value. The pipes that do not have inspection data, indicated in black, are assumed excellent for the purpose of calculation. The results show no difference in all of the rating indexes because F-PIE and W-PIE are based on PACP data and there is a lack of key parameters, only length, material, and location were used in the calculations.

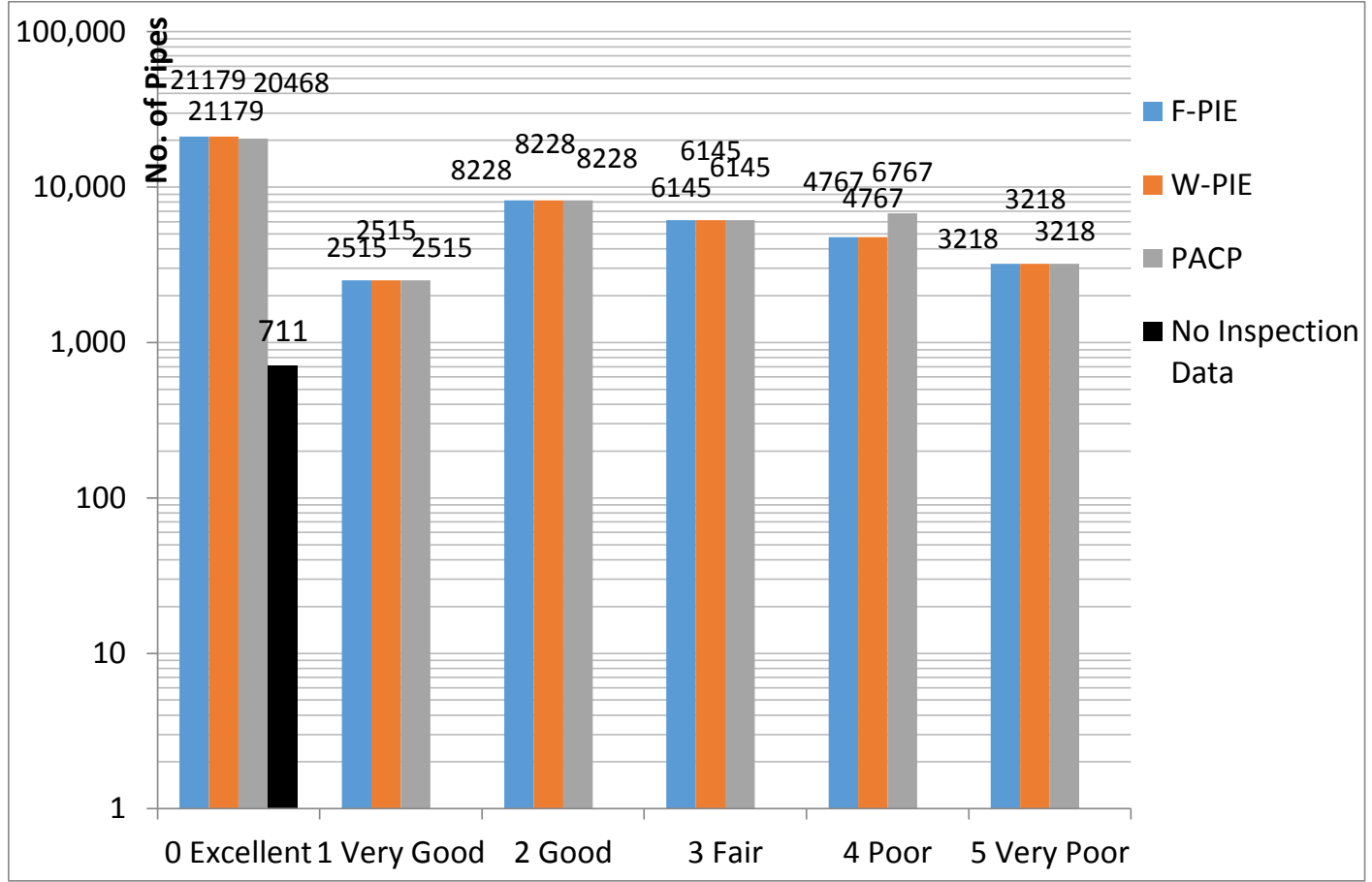

Figure G-1. Performance Index by PACP, F-PIE and W-PIE. 


\subsection{Result Comparisons}

The results calculated by W-PIE and F-PIE are compared to PACP ratings in Table G-1 and G-2 respectively. The comparison between the results from W-PIE and F-PIE is shown in Table G-3. Table G-2 shows the comparison between the F-PIE and PACP rating index, where the PACP rating is shown in row and the results from F-PIE is shown in column. The tables provide similar information as a bar chart above but in more detail. 
Table G-1. PACP Rating Index and F-PIE Comparison.

\begin{tabular}{|c|c|c|c|c|c|c|c|c|c|}
\hline & \multicolumn{6}{|c|}{ W_PIE } & \multirow[b]{2}{*}{ Total } \\
\hline & & & 0 & 1 & 2 & 3 & 4 & 5 & \\
\hline \multirow[t]{24}{*}{ PACP } & \multirow[t]{4}{*}{0} & No. of pipes & 21179 & 0 & 0 & 0 & 0 & 0 & 21179 \\
\hline & & $\%$ within $\mathrm{PACP}^{*}$ & $100.0 \%$ & $.0 \%$ & $.0 \%$ & $.0 \%$ & $.0 \%$ & $.0 \%$ & $100.0 \%$ \\
\hline & & $\%$ within $\mathrm{F} \_\mathrm{PIE}{ }^{\star *}$ & $100.0 \%$ & $.0 \%$ & $.0 \%$ & $.0 \%$ & $.0 \%$ & $.0 \%$ & $46.0 \%$ \\
\hline & & $\%$ of Total ${ }^{\star \star *}$ & $46.0 \%$ & $.0 \%$ & $.0 \%$ & $.0 \%$ & $.0 \%$ & $.0 \%$ & $46.0 \%$ \\
\hline & \multirow[t]{4}{*}{1} & No. of pipes & 0 & 2515 & 0 & 0 & 0 & 0 & 2515 \\
\hline & & $\%$ within PACP & $.0 \%$ & $100.0 \%$ & $.0 \%$ & $.0 \%$ & $.0 \%$ & $.0 \%$ & $100.0 \%$ \\
\hline & & $\%$ within F_PIE & $.0 \%$ & $100.0 \%$ & $.0 \%$ & $.0 \%$ & $.0 \%$ & $.0 \%$ & $5.5 \%$ \\
\hline & & $\%$ of Total & $.0 \%$ & $5.5 \%$ & $.0 \%$ & $.0 \%$ & $.0 \%$ & $.0 \%$ & $5.5 \%$ \\
\hline & \multirow[t]{4}{*}{2} & No. of pipes & 0 & 0 & 8228 & 0 & 0 & 0 & 8228 \\
\hline & & $\%$ within PACP & $.0 \%$ & $.0 \%$ & $100.0 \%$ & $.0 \%$ & $.0 \%$ & $.0 \%$ & $100.0 \%$ \\
\hline & & $\%$ within F_PIE & $.0 \%$ & $.0 \%$ & $100.0 \%$ & $.0 \%$ & $.0 \%$ & $.0 \%$ & $17.9 \%$ \\
\hline & & $\%$ of Total & $.0 \%$ & $.0 \%$ & $17.9 \%$ & $.0 \%$ & $.0 \%$ & $.0 \%$ & $17.9 \%$ \\
\hline & \multirow[t]{4}{*}{3} & No. of pipes & 0 & 0 & 0 & 6145 & 0 & 0 & 6145 \\
\hline & & $\%$ within PACP & $.0 \%$ & $.0 \%$ & $.0 \%$ & $100.0 \%$ & $.0 \%$ & $.0 \%$ & $100.0 \%$ \\
\hline & & $\%$ within F_PIE & $.0 \%$ & $.0 \%$ & $.0 \%$ & $100.0 \%$ & $.0 \%$ & $.0 \%$ & $13.3 \%$ \\
\hline & & $\%$ of Total & $.0 \%$ & $.0 \%$ & $.0 \%$ & $13.3 \%$ & $.0 \%$ & $.0 \%$ & $13.3 \%$ \\
\hline & \multirow[t]{4}{*}{$\overline{4}$} & No. of pipes & 0 & 0 & 0 & 0 & 4767 & 0 & 4767 \\
\hline & & $\%$ within PACP & $.0 \%$ & $.0 \%$ & $.0 \%$ & $.0 \%$ & $100.0 \%$ & $.0 \%$ & $100.0 \%$ \\
\hline & & $\%$ within F_PIE & $.0 \%$ & $.0 \%$ & $.0 \%$ & $.0 \%$ & $100.0 \%$ & $.0 \%$ & $10.4 \%$ \\
\hline & & $\%$ of Total & $.0 \%$ & $.0 \%$ & $.0 \%$ & $.0 \%$ & $10.4 \%$ & $.0 \%$ & $10.4 \%$ \\
\hline & \multirow[t]{4}{*}{5} & No. of pipes & 0 & 0 & 0 & 0 & 0 & 3218 & 3218 \\
\hline & & $\%$ within PACP & $.0 \%$ & $.0 \%$ & $.0 \%$ & $.0 \%$ & $.0 \%$ & $100.0 \%$ & $100.0 \%$ \\
\hline & & $\%$ within F_PIE & $.0 \%$ & $.0 \%$ & $.0 \%$ & $.0 \%$ & $.0 \%$ & $100.0 \%$ & $7.0 \%$ \\
\hline & & $\%$ of Total & $.0 \%$ & $.0 \%$ & $.0 \%$ & $.0 \%$ & $.0 \%$ & $7.0 \%$ & $7.0 \%$ \\
\hline \multirow{4}{*}{\multicolumn{2}{|c|}{ Total }} & No. of pipes & 21179 & 2515 & 8228 & 6145 & 4767 & 3218 & 46052 \\
\hline & & $\%$ within PACP & $46.0 \%$ & $5.5 \%$ & $17.9 \%$ & $13.3 \%$ & $10.4 \%$ & $7.0 \%$ & $100.0 \%$ \\
\hline & & $\%$ within F_PIE & $100.0 \%$ & $100.0 \%$ & $100.0 \%$ & $100.0 \%$ & $100.0 \%$ & $100.0 \%$ & $100.0 \%$ \\
\hline & & $\%$ of Total & $46.0 \%$ & $5.5 \%$ & $17.9 \%$ & $13.3 \%$ & $10.4 \%$ & $7.0 \%$ & $100.0 \%$ \\
\hline
\end{tabular}

*\% within PACP $=\frac{\text { Number of pipes }}{\text { Row } \text { Total }(\mathrm{PACP})} \times 100$

** \% within F -PIE $=\frac{\text { Number of pipes }}{\text { Column Total }(F-P I E)} \times 100$

$* * * \%$ of Total $=\frac{\text { Number of pipes }}{\text { Total }} \times 100$ 
Table G-2. PACP Rating Index and W-PIE Comparison.

\begin{tabular}{|c|c|c|c|c|c|c|c|c|c|}
\hline & \multicolumn{6}{|c|}{ W_PIE } & \multirow[b]{2}{*}{ Total } \\
\hline & & & 0 & 1 & 2 & 3 & 4 & 5 & \\
\hline \multirow[t]{24}{*}{ PACP } & \multirow[t]{4}{*}{0} & No. of pipes & 21179 & 0 & 0 & 0 & 0 & 0 & 21179 \\
\hline & & $\%$ within $\mathrm{PACP} *$ & $100.0 \%$ & $.0 \%$ & $.0 \%$ & $.0 \%$ & $.0 \%$ & $.0 \%$ & $100.0 \%$ \\
\hline & & $\%$ within W_PIE & $100.0 \%$ & $.0 \%$ & $.0 \%$ & $.0 \%$ & $.0 \%$ & $.0 \%$ & $46.0 \%$ \\
\hline & & $\%$ of Total ${ }^{\star \star *}$ & $46.0 \%$ & $.0 \%$ & $.0 \%$ & $.0 \%$ & $.0 \%$ & $.0 \%$ & $46.0 \%$ \\
\hline & \multirow[t]{4}{*}{1} & No. of pipes & 0 & 2515 & 0 & 0 & 0 & 0 & 2515 \\
\hline & & $\%$ within PACP & $.0 \%$ & $100.0 \%$ & $.0 \%$ & $.0 \%$ & $.0 \%$ & $.0 \%$ & $100.0 \%$ \\
\hline & & $\%$ within W_PIE & $.0 \%$ & $100.0 \%$ & $.0 \%$ & $.0 \%$ & $.0 \%$ & $.0 \%$ & $5.5 \%$ \\
\hline & & $\%$ of Total & $.0 \%$ & $5.5 \%$ & $.0 \%$ & $.0 \%$ & $.0 \%$ & $.0 \%$ & $5.5 \%$ \\
\hline & \multirow[t]{4}{*}{2} & No. of pipes & 0 & 0 & 8228 & 0 & 0 & 0 & 8228 \\
\hline & & $\%$ within PACP & $.0 \%$ & $.0 \%$ & $100.0 \%$ & $.0 \%$ & $.0 \%$ & $.0 \%$ & $100.0 \%$ \\
\hline & & $\%$ within W_PIE & $.0 \%$ & $.0 \%$ & $100.0 \%$ & $.0 \%$ & $.0 \%$ & $.0 \%$ & $17.9 \%$ \\
\hline & & $\%$ of Total & $.0 \%$ & $.0 \%$ & $17.9 \%$ & $.0 \%$ & $.0 \%$ & $.0 \%$ & $17.9 \%$ \\
\hline & \multirow[t]{4}{*}{$\overline{3}$} & No. of pipes & 0 & 0 & 0 & 6145 & 0 & 0 & 6145 \\
\hline & & $\%$ within PACP & $.0 \%$ & $.0 \%$ & $.0 \%$ & $100.0 \%$ & $.0 \%$ & $.0 \%$ & $100.0 \%$ \\
\hline & & $\%$ within W_PIE & $.0 \%$ & $.0 \%$ & $.0 \%$ & $100.0 \%$ & $.0 \%$ & $.0 \%$ & $13.3 \%$ \\
\hline & & $\%$ of Total & $.0 \%$ & $.0 \%$ & $.0 \%$ & $13.3 \%$ & $.0 \%$ & $.0 \%$ & $13.3 \%$ \\
\hline & \multirow[t]{4}{*}{4} & No. of pipes & 0 & 0 & 0 & 0 & 4767 & 0 & 4767 \\
\hline & & $\%$ within PACP & $.0 \%$ & $.0 \%$ & $.0 \%$ & $.0 \%$ & $100.0 \%$ & $.0 \%$ & $100.0 \%$ \\
\hline & & $\%$ within W_PIE & $.0 \%$ & $.0 \%$ & $.0 \%$ & $.0 \%$ & $100.0 \%$ & $.0 \%$ & $10.4 \%$ \\
\hline & & $\%$ of Total & $.0 \%$ & $.0 \%$ & $.0 \%$ & $.0 \%$ & $10.4 \%$ & $.0 \%$ & $10.4 \%$ \\
\hline & \multirow[t]{4}{*}{5} & No. of pipes & 0 & 0 & 0 & 0 & 0 & 3218 & 3218 \\
\hline & & $\%$ within PACP & $.0 \%$ & $.0 \%$ & $.0 \%$ & $.0 \%$ & $.0 \%$ & $100.0 \%$ & $100.0 \%$ \\
\hline & & $\%$ within W_PIE & $.0 \%$ & $.0 \%$ & $.0 \%$ & $.0 \%$ & $.0 \%$ & $100.0 \%$ & $7.0 \%$ \\
\hline & & $\%$ of Total & $.0 \%$ & $.0 \%$ & $.0 \%$ & $.0 \%$ & $.0 \%$ & $7.0 \%$ & $7.0 \%$ \\
\hline \multirow{4}{*}{\multicolumn{2}{|c|}{ Total }} & No. of pipes & 21179 & 2515 & 8228 & 6145 & 4767 & 3218 & 46052 \\
\hline & & $\%$ within PACP & $46.0 \%$ & $5.5 \%$ & $17.9 \%$ & $13.3 \%$ & $10.4 \%$ & $7.0 \%$ & $100.0 \%$ \\
\hline & & $\%$ within W_PIE & $100.0 \%$ & $100.0 \%$ & $100.0 \%$ & $100.0 \%$ & $100.0 \%$ & $100.0 \%$ & $100.0 \%$ \\
\hline & & $\%$ of Total & $46.0 \%$ & $5.5 \%$ & $17.9 \%$ & $13.3 \%$ & $10.4 \%$ & $7.0 \%$ & $100.0 \%$ \\
\hline
\end{tabular}

\footnotetext{
*\% within PACP $=\frac{\text { Number of pipes }}{\text { Row Total }(P A C P)} \times 100$

** \% within $W-P I E=\frac{\text { Number of pipes }}{\text { Column Total }(W-P I E)} \times 100$

*** \% of Total $=\frac{\text { Number of pipes }}{\text { Total }} \times 100$
} 
Table G-3. W-PIE and F-PIE Comparison.

\begin{tabular}{|c|c|c|c|c|c|c|c|c|c|}
\hline & \multicolumn{6}{|c|}{ F_PIE } & \multirow[b]{2}{*}{ Total } \\
\hline & & & 0 & 1 & 2 & 3 & 4 & 5 & \\
\hline \multirow[t]{24}{*}{ W_PIE } & \multirow[t]{4}{*}{0} & No. of pipes & 19237 & 1819 & 123 & 0 & 0 & 0 & 21179 \\
\hline & & \% within W_PIE & $90.8 \%$ & $8.6 \%$ & $.6 \%$ & $.0 \%$ & $.0 \%$ & $.0 \%$ & $100.0 \%$ \\
\hline & & $\%$ within F_PIE & $100.0 \%$ & $42.2 \%$ & $1.5 \%$ & $.0 \%$ & $.0 \%$ & $.0 \%$ & $46.0 \%$ \\
\hline & & $\%$ of Total & $41.8 \%$ & $3.9 \%$ & $.3 \%$ & $.0 \%$ & $.0 \%$ & $.0 \%$ & $46.0 \%$ \\
\hline & \multirow[t]{4}{*}{1} & No. of pipes & 0 & 2492 & 23 & 0 & 0 & 0 & 2515 \\
\hline & & $\%$ within W_PIE & $.0 \%$ & $99.1 \%$ & $.9 \%$ & $.0 \%$ & $.0 \%$ & $.0 \%$ & $100.0 \%$ \\
\hline & & $\%$ within F_PIE & $.0 \%$ & $57.8 \%$ & $.3 \%$ & $.0 \%$ & $.0 \%$ & $.0 \%$ & $5.5 \%$ \\
\hline & & $\%$ of Total & $.0 \%$ & $5.4 \%$ & $.0 \%$ & $.0 \%$ & $.0 \%$ & $.0 \%$ & $5.5 \%$ \\
\hline & \multirow[t]{4}{*}{2} & No. of pipes & 0 & 0 & 8190 & 38 & 0 & 0 & 8228 \\
\hline & & $\%$ within W_PIE & $.0 \%$ & $.0 \%$ & $99.5 \%$ & $.5 \%$ & $.0 \%$ & $.0 \%$ & $100.0 \%$ \\
\hline & & $\%$ within F_PIE & $.0 \%$ & $.0 \%$ & $98.2 \%$ & $.6 \%$ & $.0 \%$ & $.0 \%$ & $17.9 \%$ \\
\hline & & $\%$ of Total & $.0 \%$ & $.0 \%$ & $17.8 \%$ & $.1 \%$ & $.0 \%$ & $.0 \%$ & $17.9 \%$ \\
\hline & \multirow[t]{4}{*}{3} & No. of pipes & 0 & 0 & 0 & 6116 & 29 & 0 & 6145 \\
\hline & & $\%$ within W_PIE & $.0 \%$ & $.0 \%$ & $.0 \%$ & $99.5 \%$ & $.5 \%$ & $.0 \%$ & $100.0 \%$ \\
\hline & & $\%$ within F_PIE & $.0 \%$ & $.0 \%$ & $.0 \%$ & $99.4 \%$ & $.6 \%$ & $.0 \%$ & $13.3 \%$ \\
\hline & & $\%$ of Total & $.0 \%$ & $.0 \%$ & $.0 \%$ & $13.3 \%$ & $.1 \%$ & $.0 \%$ & $13.3 \%$ \\
\hline & \multirow[t]{4}{*}{4} & No. of pipes & 0 & 0 & 0 & 0 & 4767 & 0 & 4767 \\
\hline & & $\%$ within W_PIE & $.0 \%$ & $.0 \%$ & $.0 \%$ & $.0 \%$ & $100.0 \%$ & $.0 \%$ & $100.0 \%$ \\
\hline & & $\%$ within F_PIE & $.0 \%$ & $.0 \%$ & $.0 \%$ & $.0 \%$ & $99.4 \%$ & $.0 \%$ & $10.4 \%$ \\
\hline & & $\%$ of Total & $.0 \%$ & $.0 \%$ & $.0 \%$ & $.0 \%$ & $10.4 \%$ & $.0 \%$ & $10.4 \%$ \\
\hline & \multirow[t]{4}{*}{5} & No. of pipes & 0 & 0 & 0 & 0 & 0 & 3218 & 3218 \\
\hline & & $\%$ within W_PIE & $.0 \%$ & $.0 \%$ & $.0 \%$ & $.0 \%$ & $.0 \%$ & $100.0 \%$ & $100.0 \%$ \\
\hline & & $\%$ within F_PIE & $.0 \%$ & $.0 \%$ & $.0 \%$ & $.0 \%$ & $.0 \%$ & $100.0 \%$ & $7.0 \%$ \\
\hline & & $\%$ of Total & $.0 \%$ & $.0 \%$ & $.0 \%$ & $.0 \%$ & $.0 \%$ & $7.0 \%$ & $7.0 \%$ \\
\hline \multirow{4}{*}{\multicolumn{2}{|c|}{ Total }} & No. of pipes & 19237 & 4311 & 8336 & 6154 & 4796 & 3218 & 46052 \\
\hline & & $\%$ within W_PIE & $41.8 \%$ & $9.4 \%$ & $18.1 \%$ & $13.4 \%$ & $10.4 \%$ & $7.0 \%$ & $100.0 \%$ \\
\hline & & $\%$ within F_PIE & $100.0 \%$ & $100.0 \%$ & $100.0 \%$ & $100.0 \%$ & $100.0 \%$ & $100.0 \%$ & $100.0 \%$ \\
\hline & & $\%$ of Total & $41.8 \%$ & $9.4 \%$ & $18.1 \%$ & $13.4 \%$ & $10.4 \%$ & $7.0 \%$ & $100.0 \%$ \\
\hline
\end{tabular}

$* \%$ within $W-P I E=\frac{\text { Number of pipes }}{\text { Row } \operatorname{Total}(W-P I E)} \times 100$

** \% within $F-P I E=\frac{\text { Number of pipes }}{\text { Column Total }(F-P I E)} \times 100$

$* * * \%$ of Total $=\frac{\text { Number of pipes }}{\text { Total }} \times 100$ 


\section{City C}

We analyzed data received from City $\mathrm{C}$ and evaluated each pipe section (manhole to manhole) using performance models (weighted factor and fuzzy inference system). City C has given its database which is downloaded through FTP site to the research group at Virginia Tech. Altogether there were 15 geo-database tables which mainly consist of City $\mathrm{C}$ inspection and maintenance records, sewer main table and manhole table. More detail about the data supplied by City $\mathrm{C}$ can be found in appendix F. City $\mathrm{C}$ is in an early stage of developing its wastewater pipe database and we was provided with the data including seven selected parameters used in this pilot study.

\section{Parameters}
a. Pipe age
b. Pipe diameter
c. Pipe depth
d. Pipe slope
e. Pipe length
f. Pipe material
g. Pipe condition (inspection records)

Pipe age, pipe diameter, pipe depth, pipe slope, pipe length, and pipe material are stored as attributes in mainline table. Pipe condition was calculated from inspection records (CCTV and dye test). The inspection records are coded using PACP. Based on limited data we received, the performance models were run with the results presenting below.

\subsection{W-PIE and F-PIE Results}

Figure G-2 shows the bar chart of the performance index calculated by PACP, W-PIE and F-PIE to illustrate the distribution of the results, which the $\mathrm{x}$-axis is the performance index and $y$-axis is the number of pipes that fall within each value.

The results show that most of the pipes are 0,1 , and 2 which are in excellent, very good, and good respectively. Fair (3), poor (4), and very poor (5) pipes account for approximately 2 percent of entire wastewater pipe system. Please note there are 31,833 pipes that do not have inspection records, therefore, there is a higher percentage of excellent and good pipes which probably overstate the actual condition of the pipes in the field. The pipes that do not have inspection data, indicated in black, are assumed excellent for the purpose of calculation. There is little difference in the number of fair, and poor pipes in the results provided by F-PIE, W-PIE, No PACP data, and PACP. However, results from F-PIE and W-PIE show more very good and good pipes, indicating that these pipes are shifted from excellent because of the knowledge base. 


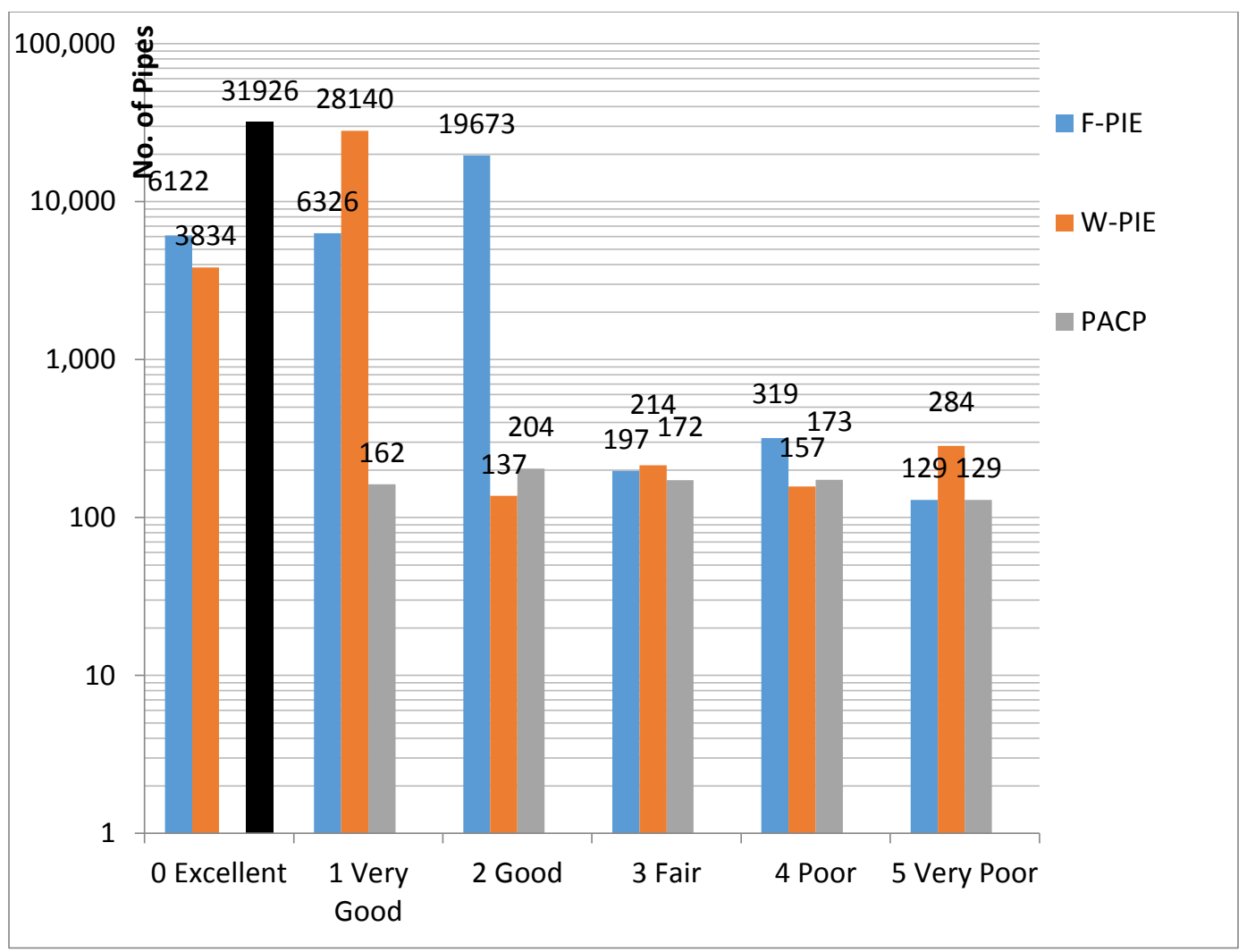

Figure G-2. Performance Index by No PACP Data, PACP, F-PIE and W-PIE.

\subsection{Result Comparisons}

The results calculated by W-PIE and F-PIE are compared to PACP ratings, provided by City $\mathrm{C}$ in Tables G-4, and G-5 respectively, and the comparison of the results from the weighted factor and fuzzy inference models shown in Table G-6. The tables provide similar information as the bar chart above but in detail. Table G-4 shows the comparison between the F-PIE and PACP rating index, where the PACP rating index is shown in rows and the results from F-PIE are shown in columns. For example in the third row; the number in the fourth column of the third row is 168 , which indicates that there are 168 pipe sections that rated 2 by PACP and rated 3 by F-PIE. The total number of the pipe sections which rated 2 by PACP is 204 and thus $\%$ of total is $0.6 \%$. In the same manner, the total number of the pipe sections which rated 3 by F-PIE is 197 and thus $\%$ of total is $0.6 \%$.

From the results present in Tables G-4 and G-5, both F-PIE and W-PIE always give higher numbers compared to PACP because both models use additional parameters. The author also would like to point out that, even though the PACP rating index is not available, which can imply that the inspection has not been performed, both F-PIE and W-PIE are able to calculate and score the pipes. 
Table G-4. PACP Rating Index and F-PIE Comparison.

\begin{tabular}{|c|c|c|c|c|c|c|c|c|c|}
\hline & \multicolumn{6}{|c|}{ F_PIE } & \multirow[b]{2}{*}{ Total } \\
\hline & & & 0 & 1 & 2 & 3 & 4 & 5 & \\
\hline \multirow{4}{*}{\multicolumn{2}{|c|}{$\begin{array}{l}\text { PACP } .0 \text { (No } \\
\text { PACP data) }\end{array}$}} & No. of pipes & 6122 & 6278 & 19526 & 0 & 0 & 0 & 31926 \\
\hline & & $\%$ within PACP & $19.2 \%$ & $19.7 \%$ & $61.2 \%$ & $.0 \%$ & $.0 \%$ & $.0 \%$ & $100.0 \%$ \\
\hline & & $\%$ within F_PIE & $100.0 \%$ & $99.2 \%$ & $99.3 \%$ & $.0 \%$ & $.0 \%$ & $.0 \%$ & $97.4 \%$ \\
\hline & & $\%$ of Total & $18.7 \%$ & $19.2 \%$ & $59.6 \%$ & $.0 \%$ & $.0 \%$ & $.0 \%$ & $97.4 \%$ \\
\hline \multirow{4}{*}{\multicolumn{2}{|c|}{1}} & No. of pipes & 0 & 48 & 111 & 3 & 0 & 0 & 162 \\
\hline & & $\%$ within PACP & $.0 \%$ & $29.6 \%$ & $68.5 \%$ & $1.9 \%$ & $.0 \%$ & $.0 \%$ & $100.0 \%$ \\
\hline & & $\%$ within F_PIE & $.0 \%$ & $.8 \%$ & $.6 \%$ & $1.5 \%$ & $.0 \%$ & $.0 \%$ & $.5 \%$ \\
\hline & & $\%$ of Total & $.0 \%$ & $.1 \%$ & $.3 \%$ & $.0 \%$ & $.0 \%$ & $.0 \%$ & $.5 \%$ \\
\hline \multirow{4}{*}{\multicolumn{2}{|c|}{2}} & No. of pipes & 0 & 0 & 36 & 168 & 0 & 0 & 204 \\
\hline & & $\%$ within PACP & $.0 \%$ & $.0 \%$ & $17.6 \%$ & $82.4 \%$ & $.0 \%$ & $.0 \%$ & $100.0 \%$ \\
\hline & & $\%$ within F_PIE & $.0 \%$ & $.0 \%$ & $.2 \%$ & $85.3 \%$ & $.0 \%$ & $.0 \%$ & $.6 \%$ \\
\hline & & $\%$ of Total & $.0 \%$ & $.0 \%$ & $.1 \%$ & $.5 \%$ & $.0 \%$ & $.0 \%$ & $.6 \%$ \\
\hline \multirow{4}{*}{\multicolumn{2}{|c|}{3}} & No. of pipes & 0 & 0 & 0 & 26 & 146 & 0 & 172 \\
\hline & & $\%$ within PACP & $.0 \%$ & $.0 \%$ & $.0 \%$ & $15.1 \%$ & $84.9 \%$ & $.0 \%$ & $100.0 \%$ \\
\hline & & $\%$ within F_PIE & $.0 \%$ & $.0 \%$ & $.0 \%$ & $13.2 \%$ & $45.8 \%$ & $.0 \%$ & $.5 \%$ \\
\hline & & $\%$ of Total & $.0 \%$ & $.0 \%$ & $.0 \%$ & $.1 \%$ & $.4 \%$ & $.0 \%$ & $.5 \%$ \\
\hline \multirow{4}{*}{\multicolumn{2}{|c|}{4}} & No. of pipes & 0 & 0 & 0 & 0 & 173 & 0 & 173 \\
\hline & & $\%$ within PACP & $.0 \%$ & $.0 \%$ & $.0 \%$ & $.0 \%$ & $100.0 \%$ & $.0 \%$ & $100.0 \%$ \\
\hline & & $\%$ within F_PIE & $.0 \%$ & $.0 \%$ & $.0 \%$ & $.0 \%$ & $54.2 \%$ & $.0 \%$ & $.5 \%$ \\
\hline & & $\%$ of Total & $.0 \%$ & $.0 \%$ & $.0 \%$ & $.0 \%$ & $.5 \%$ & $.0 \%$ & $.5 \%$ \\
\hline \multirow{4}{*}{\multicolumn{2}{|c|}{5}} & No. of pipes & 0 & 0 & 0 & 0 & 0 & 129 & 129 \\
\hline & & $\%$ within PACP & $.0 \%$ & $.0 \%$ & $.0 \%$ & $.0 \%$ & $.0 \%$ & $100.0 \%$ & $100.0 \%$ \\
\hline & & $\%$ within F_PIE & $.0 \%$ & $.0 \%$ & $.0 \%$ & $.0 \%$ & $.0 \%$ & $100.0 \%$ & $.4 \%$ \\
\hline & & $\%$ of Total & $.0 \%$ & $.0 \%$ & $.0 \%$ & $.0 \%$ & $.0 \%$ & $.4 \%$ & $.4 \%$ \\
\hline \multirow{4}{*}{\multicolumn{2}{|c|}{ Total }} & No. of pipes & 6122 & 6326 & 19673 & 197 & 319 & 129 & 32766 \\
\hline & & $\%$ within PACP & $18.7 \%$ & $19.3 \%$ & $60.0 \%$ & $.6 \%$ & $1.0 \%$ & $.4 \%$ & $100.0 \%$ \\
\hline & & $\%$ within $F_{-}$PIE & $100.0 \%$ & $100.0 \%$ & $100.0 \%$ & $100.0 \%$ & $100.0 \%$ & $100.0 \%$ & $100.0 \%$ \\
\hline & & $\%$ of Total & $18.7 \%$ & $19.3 \%$ & $60.0 \%$ & $.6 \%$ & $1.0 \%$ & $.4 \%$ & $100.0 \%$ \\
\hline
\end{tabular}

$* \%$ within PACP $=\frac{\text { Number of pipes }}{\text { Row } \text { Total }(P A C P)} \times 100$

** \% within F - PIE $=\frac{\text { Number of pipes }}{\text { Column } \operatorname{Total}(F-P I E)} \times 100$

*** \% of Total $=\frac{\text { Number of pipes }}{\text { Total }} \times 100$ 
Table G-5. PACP Rating Index and W-PIE Comparison.

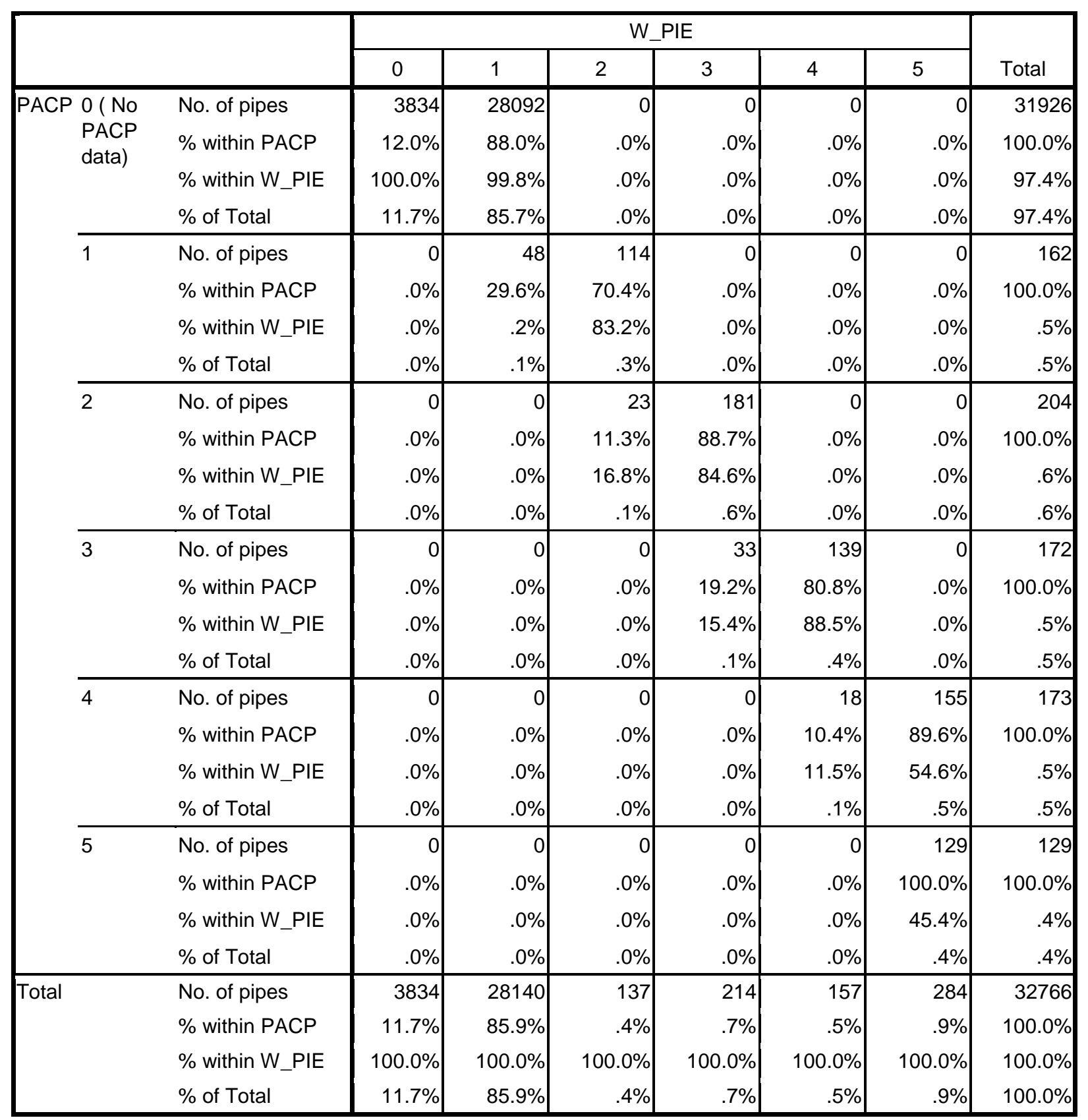

* \% within PACP $=\frac{\text { Number of pipes }}{\text { Row } \text { Total }(P A C P)} \times 100$

$* * \%$ within $W-P I E=\frac{\text { Number of pipes }}{\text { Column Total }(W-P I E)} \times 100$

$* * * \%$ of Total $=\frac{\text { Number of pipes }}{\text { Total }} \times 100$ 
Table G-6. W-PIE and F-PIE Comparison.

\begin{tabular}{|c|c|c|c|c|c|c|c|c|c|}
\hline & \multicolumn{6}{|c|}{ F_PIE } & \multirow[b]{2}{*}{ Total } \\
\hline & & & .0 & 1 & 2 & 3 & 4 & 5 & \\
\hline \multirow[t]{24}{*}{ W_PIE } & \multirow[t]{4}{*}{0} & No. of pipes & 403 & 2984 & 812 & 0 & 0 & 0 & 4199 \\
\hline & & $\%$ within W_PIE* & $9.6 \%$ & $71.1 \%$ & $19.3 \%$ & $.0 \%$ & $.0 \%$ & $.0 \%$ & $100.0 \%$ \\
\hline & & $\%$ within F_PIE ${ }^{\star *}$ & $11.5 \%$ & $34.0 \%$ & $4.1 \%$ & $.0 \%$ & $.0 \%$ & $.0 \%$ & $12.8 \%$ \\
\hline & & $\%$ of Total ${ }^{* \star *}$ & $1.2 \%$ & $9.1 \%$ & $2.5 \%$ & $.0 \%$ & $.0 \%$ & $.0 \%$ & $12.8 \%$ \\
\hline & \multirow[t]{4}{*}{1} & No. of pipes & 3109 & 5790 & 18865 & 194 & 0 & 0 & 27958 \\
\hline & & \% within W_PIE & $11.1 \%$ & $20.7 \%$ & $67.5 \%$ & $.7 \%$ & $.0 \%$ & $.0 \%$ & $100.0 \%$ \\
\hline & & $\%$ within F_PIE & $88.5 \%$ & $65.9 \%$ & $95.4 \%$ & $63.2 \%$ & $.0 \%$ & $.0 \%$ & $85.3 \%$ \\
\hline & & $\%$ of Total & $9.5 \%$ & $17.7 \%$ & $57.6 \%$ & $.6 \%$ & $.0 \%$ & $.0 \%$ & $85.3 \%$ \\
\hline & \multirow[t]{4}{*}{2} & No. of pipes & 0 & 14 & 85 & 11 & 0 & 0 & 110 \\
\hline & & \% within W_PIE & $.0 \%$ & $12.7 \%$ & $77.3 \%$ & $10.0 \%$ & $.0 \%$ & $.0 \%$ & $100.0 \%$ \\
\hline & & $\%$ within F_PIE & $.0 \%$ & $.2 \%$ & $.4 \%$ & $3.6 \%$ & $.0 \%$ & $.0 \%$ & $.3 \%$ \\
\hline & & $\%$ of Total & $.0 \%$ & $.0 \%$ & $.3 \%$ & $.0 \%$ & $.0 \%$ & $.0 \%$ & $.3 \%$ \\
\hline & \multirow[t]{4}{*}{3} & No. of pipes & 0 & 0 & 6 & 93 & 0 & 0 & 99 \\
\hline & & \% within W_PIE & $.0 \%$ & $.0 \%$ & $6.1 \%$ & $93.9 \%$ & $.0 \%$ & $.0 \%$ & $100.0 \%$ \\
\hline & & $\%$ within F_PIE & $.0 \%$ & $.0 \%$ & $.0 \%$ & $30.3 \%$ & $.0 \%$ & $.0 \%$ & $.3 \%$ \\
\hline & & $\%$ of Total & $.0 \%$ & $.0 \%$ & $.0 \%$ & $.3 \%$ & $.0 \%$ & $.0 \%$ & $.3 \%$ \\
\hline & \multirow[t]{4}{*}{4} & No. of pipes & 0 & 0 & 0 & 9 & 169 & 0 & 178 \\
\hline & & $\%$ within W_PIE & $.0 \%$ & $.0 \%$ & $.0 \%$ & $5.1 \%$ & $94.9 \%$ & $.0 \%$ & $100.0 \%$ \\
\hline & & $\%$ within F_PIE & $.0 \%$ & $.0 \%$ & $.0 \%$ & $2.9 \%$ & $54.5 \%$ & $.0 \%$ & $.5 \%$ \\
\hline & & $\%$ of Total & $.0 \%$ & $.0 \%$ & $.0 \%$ & $.0 \%$ & $.5 \%$ & $.0 \%$ & $.5 \%$ \\
\hline & \multirow[t]{4}{*}{5} & No. of pipes & 0 & 0 & 0 & 0 & 141 & 80 & 221 \\
\hline & & \% within W_PIE & $.0 \%$ & $.0 \%$ & $.0 \%$ & $.0 \%$ & $63.8 \%$ & $36.2 \%$ & $100.0 \%$ \\
\hline & & $\%$ within F_PIE & $.0 \%$ & $.0 \%$ & $.0 \%$ & $.0 \%$ & $45.5 \%$ & $100.0 \%$ & $.7 \%$ \\
\hline & & $\%$ of Total & $.0 \%$ & $.0 \%$ & $.0 \%$ & $.0 \%$ & $.4 \%$ & $.2 \%$ & $.7 \%$ \\
\hline \multirow{4}{*}{\multicolumn{2}{|c|}{ Total }} & No. of pipes & 3512 & 8788 & 19768 & 307 & 310 & 80 & 32765 \\
\hline & & \% within W_PIE & $10.7 \%$ & $26.8 \%$ & $60.3 \%$ & $.9 \%$ & $.9 \%$ & $.2 \%$ & $100.0 \%$ \\
\hline & & $\%$ within F_PIE & $100.0 \%$ & $100.0 \%$ & $100.0 \%$ & $100.0 \%$ & $100.0 \%$ & $100.0 \%$ & $100.0 \%$ \\
\hline & & $\%$ of Total & $10.7 \%$ & $26.8 \%$ & $60.3 \%$ & $.9 \%$ & $.9 \%$ & $.2 \%$ & $100.0 \%$ \\
\hline
\end{tabular}

$* \%$ within $W-P I E=\frac{\text { Number of pipes }}{\text { Row } \operatorname{Total}(W-P I E)} \times 100$

$* * \%$ within $F-P I E=\frac{\text { Number of pipes }}{\text { Column } \operatorname{Total}(F-P I E)} \times 100$

$* * * \%$ of Total $=\frac{\text { Number of pipes }}{\text { Total }} \times 100$ 


\section{City D}

We analyzed data received from City D and evaluated each pipe section (manhole to Manahole-MH) using performance models (weighted factor and fuzzy inference system). There were 13 shape-files which mainly consist of pipelines, manhole, and land use along with soil and city limits. More detail about the data supplied by City D can be found in appendix F. City D is in process of transferring all pipe inspection records, which typically had been stored in folders and DVD, to the geo-database system. There were 6 parameters that used in this study.

\section{Parameters}
a. Pipe age
b. Pipe diameter
c. Pipe depth
d. Pipe slope
e. Pipe length
f. Pipe material

The parameters above are stored as attributes in a mainline table. There are approximately 200 pipes where the ages are known. The performance models were run with the limited data available. The results are shown below.

\subsection{W-PIE and F-PIE Results}

Figure G-3 illustrates the bar chart of the performance index calculated by no PACP data, W-PIE, and F-PIE to illustrate the distribution of the results, where the $\mathrm{x}$-axis is the performance index and y-axis is the number of pipes that fall within each value. The pipes that do not have inspection data which indicated in black color are assumed excellent for the purpose of calculation.

The results from both W-PIE and F-PIE show that all of the pipes are 0, 1, and 2 which are in excellent, very good, and good respectively. There are no Fair (3), poor (4), and very poor (5) pipes in this evaluation because of the lack of key parameters such as pipe condition. The main parameter that plays a role here is the pipe slope. We observed that pipe slopes are substantially lower compared to other utilities, this may due to the geography of City D, or inaccuracy of the derived data. The results from F-PIE and W-PIE show more very good and good pipes which indicate these pipes are shifted from excellent pipes because of the knowledge base. 


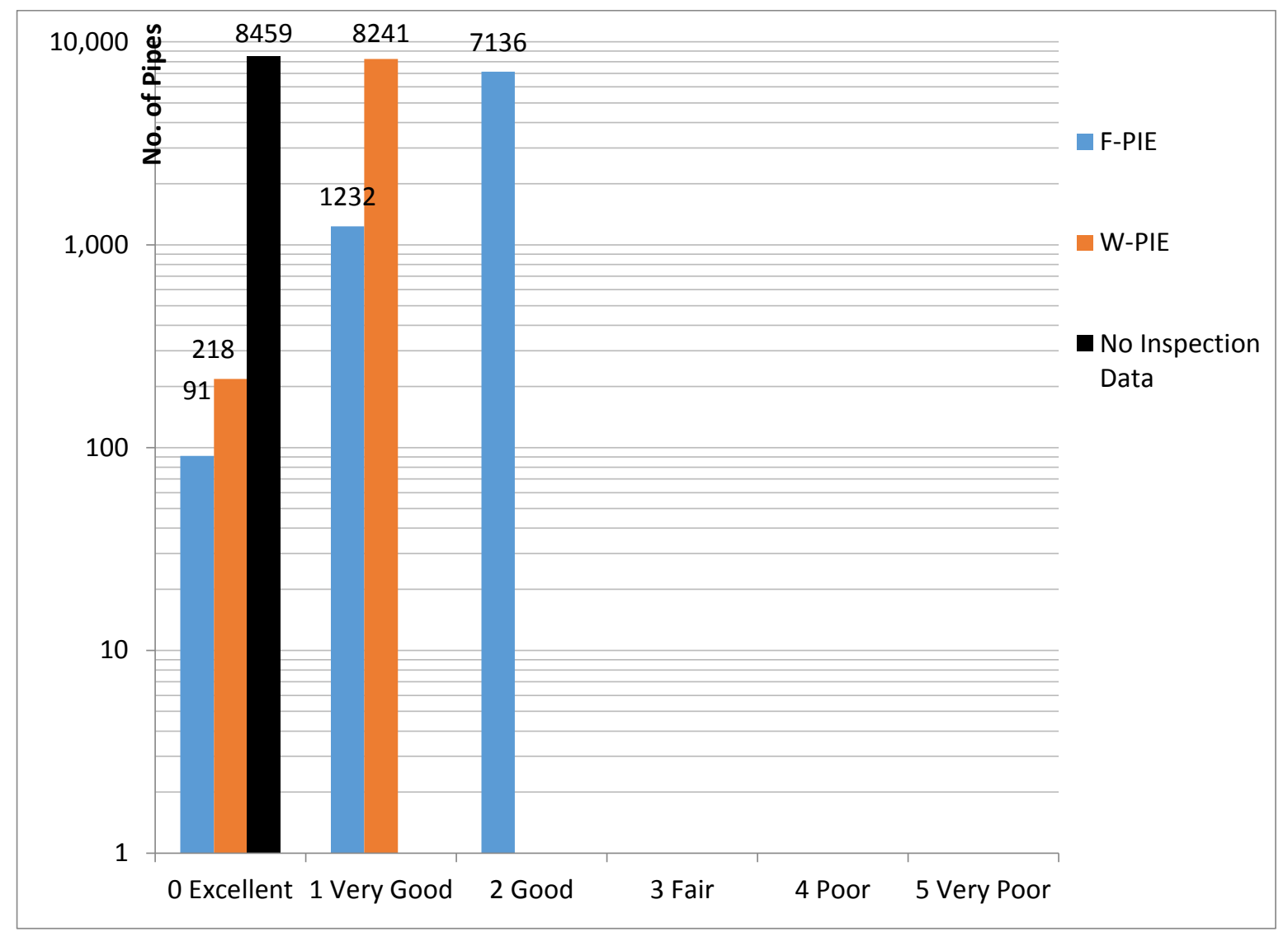

Figure G-3. Performance Index by No PACP Data, F-PIE and W-PIE.

\subsection{Result Comparisons}

The results calculated by weighted factor and fuzzy inference models are compared to PACP rating in Table G-7, and Table G-8. Table G-7 shows the results obtained by F-PIE comparing to the PACP rating index where the PACP rating index are in rows, and the results from F-PIE are in columns. Table G-8 shows the performance index calculated from weighted factors comparing to the PACP rating index and Table G-9 shows the comparison of the results between weighted factor and fuzzy inference models. The tables provide similar information as a bar chart above but in detail. 
Table G-7. PACP and F-PIE Comparison.

\begin{tabular}{|c|c|c|c|c|c|}
\hline & \multicolumn{3}{|c|}{ F_PIE } & \multirow[b]{2}{*}{ Total } \\
\hline & & 0 & 1 & 2 & \\
\hline \multirow{4}{*}{$\begin{array}{c}\text { PACP } 0 \text { (No PACP } \\
\text { data) }\end{array}$} & No. of pipes & 91 & 1232 & 7136 & 8459 \\
\hline & $\%$ within PACP* & $1.1 \%$ & $14.6 \%$ & $84.4 \%$ & $100.0 \%$ \\
\hline & $\begin{array}{l}\text { \% within } \\
\text { F_PIE** }\end{array}$ & $100.0 \%$ & $100.0 \%$ & $100.0 \%$ & $100.0 \%$ \\
\hline & $\%$ of Total ${ }^{* \star *}$ & $1.1 \%$ & $14.6 \%$ & $84.4 \%$ & $100.0 \%$ \\
\hline \multirow[t]{4}{*}{ Total } & No. of pipes & 91 & 1232 & 7136 & 8459 \\
\hline & $\%$ within PACP & $1.1 \%$ & $14.6 \%$ & $84.4 \%$ & $100.0 \%$ \\
\hline & \% within F_PIE & $100.0 \%$ & $100.0 \%$ & $100.0 \%$ & $100.0 \%$ \\
\hline & $\%$ of Total & $1.1 \%$ & $14.6 \%$ & $84.4 \%$ & $100.0 \%$ \\
\hline
\end{tabular}

*\% within PACP $=\frac{\text { Number of pipes }}{\text { Row } \text { Total }(P A C P)} \times 100$

** \% within $F-P I E=\frac{\text { Number of pipes }}{\text { Column } \operatorname{Total}(F-P I E)} \times 100$

$* * * \%$ of Total $=\frac{\text { Number of pipes }}{\text { Total }} \times 100$

Table G-8. PACP and W-PIE Comparison.

\begin{tabular}{|c|c|c|c|c|c|}
\hline & \multicolumn{2}{|c|}{ W_PIE } & \multirow[b]{2}{*}{ Total } \\
\hline & & & 0 & 1 & \\
\hline \multirow[t]{4}{*}{ PACP } & \multirow{4}{*}{$\begin{array}{l}0 \text { (No PACP } \\
\text { data) }\end{array}$} & No. of pipes & 218 & 8241 & 8459 \\
\hline & & $\%$ within PACP* & $2.6 \%$ & $97.4 \%$ & $100.0 \%$ \\
\hline & & $\%$ within W_PIE & $100.0 \%$ & $100.0 \%$ & $100.0 \%$ \\
\hline & & $\%$ of Total ${ }^{\star \star *}$ & $2.6 \%$ & $97.4 \%$ & $100.0 \%$ \\
\hline \multirow{4}{*}{\multicolumn{2}{|c|}{ Total }} & No. of pipes & 218 & 8241 & 8459 \\
\hline & & $\%$ within PACP & $2.6 \%$ & $97.4 \%$ & $100.0 \%$ \\
\hline & & $\%$ within W_PIE & $100.0 \%$ & $100.0 \%$ & $100.0 \%$ \\
\hline & & $\%$ of Total & $2.6 \%$ & $97.4 \%$ & $100.0 \%$ \\
\hline
\end{tabular}

* \% within PACP $=\frac{\text { Number of pipes }}{\text { Row } \text { Total }(P A C P)} \times 100$

** $\%$ within $W-P I E=\frac{\text { Number of pipes }}{\text { Column Total }(W-P I E)} \times 100$

*** \% of Total $=\frac{\text { Number of pipes }}{\text { Total }} \times 100$ 
Table G-9. W-PIE and F-PIE Comparison.

\begin{tabular}{|c|c|c|c|c|c|c|}
\hline & & & \multicolumn{3}{|c|}{ F_PIE } & \multirow[b]{2}{*}{ Total } \\
\hline & & & 0 & 1 & 2 & \\
\hline \multirow[t]{8}{*}{ W_PIE } & 0 & No. of pipes & 57 & 35 & 126 & 218 \\
\hline & & \% within W_PIE* & $26.1 \%$ & $16.1 \%$ & $57.8 \%$ & $100.0 \%$ \\
\hline & & $\%$ within $\mathrm{F} \_\mathrm{PIE}{ }^{* *}$ & $62.6 \%$ & $2.8 \%$ & $1.8 \%$ & $2.6 \%$ \\
\hline & & $\%$ of Total ${ }^{* * *}$ & $.7 \%$ & $.4 \%$ & $1.5 \%$ & $2.6 \%$ \\
\hline & 1 & No. of pipes & 34 & 1197 & 7010 & 8241 \\
\hline & & \% within W_PIE & $.4 \%$ & $14.5 \%$ & $85.1 \%$ & $100.0 \%$ \\
\hline & & $\%$ within F_PIE & $37.4 \%$ & $97.2 \%$ & $98.2 \%$ & $97.4 \%$ \\
\hline & & $\%$ of Total & $.4 \%$ & $14.2 \%$ & $82.9 \%$ & $97.4 \%$ \\
\hline \multirow{4}{*}{\multicolumn{2}{|c|}{ Total }} & No. of pipes & 91 & 1232 & 7136 & 8459 \\
\hline & & $\%$ within W_PIE & $1.1 \%$ & $14.6 \%$ & $84.4 \%$ & $100.0 \%$ \\
\hline & & $\%$ within F_PIE & $100.0 \%$ & $100.0 \%$ & $100.0 \%$ & $100.0 \%$ \\
\hline & & $\%$ of Total & $1.1 \%$ & $14.6 \%$ & $84.4 \%$ & $100.0 \%$ \\
\hline
\end{tabular}

$* \%$ within $W-P I E=\frac{\text { Number of pipes }}{\text { Row } \operatorname{Total}(W-P I E)} \times 100$

$* * \%$ within $F-P I E=\frac{\text { Number of pipes }}{\text { Column } \operatorname{Total}(F-P I E)} \times 100$

$* * * \%$ of Total $=\frac{\text { Number of pipes }}{\text { Total }} \times 100$ 


\section{City $E$}

The City E gave data in the form of eight shape-files. These consist primarily of the main pipelines, manholes, junctions, and diameter changes. The sewer main shape file consists of all the sewer mains in City E city limits which are spatially mapped in an undefined projected coordinate system. The sewer main attribute table consists of various pipe parameters such as Pipe ID, pipe shape, diameter, material, length, installation year, and elevation of the upstream and downstream nodes. Although a few columns were left blank, enough information was collected for around 44,000 pipes. More detail about the data supplied by City E can be found in appendix E.

There was a soil field in the database which had 19 soil classifications associated with the pipe segments. These soil classifications were reviewed for their characteristics in the "Soil Survey of the County" report. The initial analysis was conducted to determine which of the 19 soil types statistically contributed to the poor condition pipes.

There were six parameters that used in this study.

\section{Parameters}
a. Pipe age
b. Pipe diameter
c. Pipe length
d. Pipe material
e. Soil type
f. Pipe condition (Inspection Records)

The recommendation to City E was to use the areas identified for a detailed analysis. These smaller areas have more data allowing for additional parameters to be entered into the models. Specific data on sewer backups, SSO identified by stretch, depth of sewers, traffic loading, and maintenance problems from the CMMS can be added to the analysis. By using these smaller pilot areas, it is easier to gather. A better recommendation could then be developed identifying which data is most important and should be collected to assist in future prioritization of repair, rehabilitation and replacement by City E. 


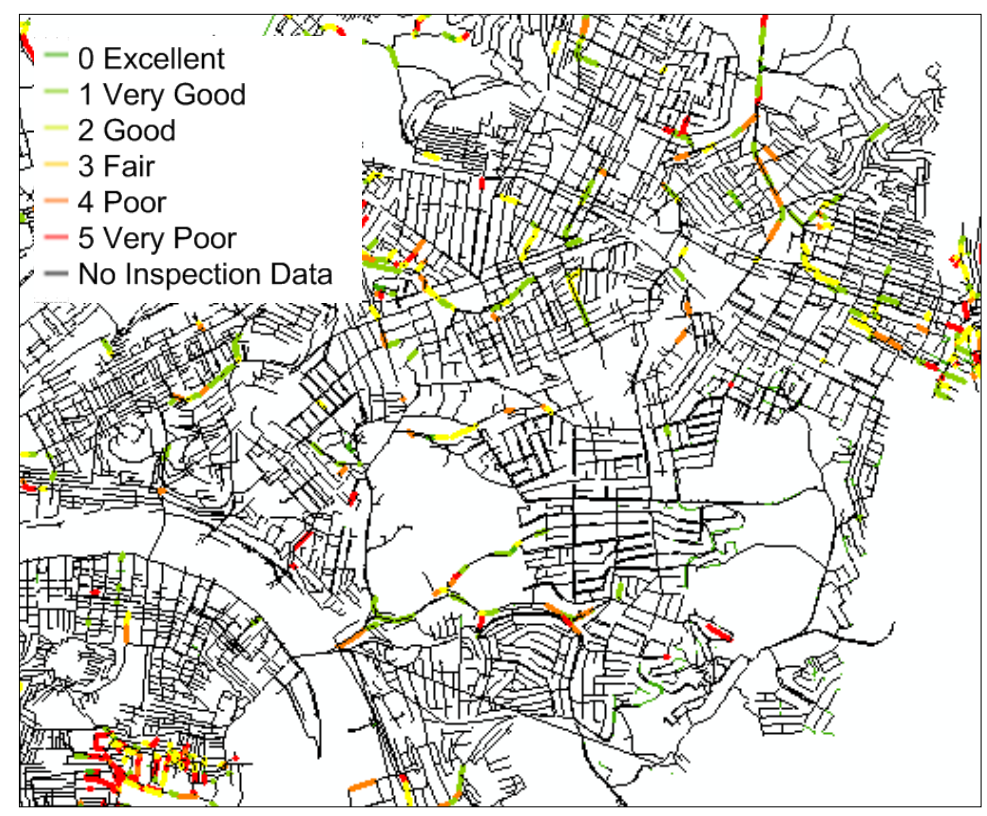

Figure G-4. PACP Rating Index.

Figure G-4 presents the PACP rating index for City E. The pipes are rated based on scale (0-5) where 0 (green) represents excellent and 5 (red) failed. The pipes that do not have inspection data (0), indicated in black, are assumed excellent for the purpose of calculation. Based on the data received, the models were run using both W-PIE and F-PIE.

\subsection{W-PIE and F-PIE Results}

Figure G-5 shows the result of the performance index calculated by the weighted factor model where 0 indicates excellent and 5 indicates very poor. The result was mapped to GIS to illustrate the distribution of the performance index. As shown in Figure G-5, the majority of the performance index for City $\mathrm{E}$ is 0 and 1, which indicate excellent and good, respectively. From the results presented here, the failed and poor pipes are concentrated along the center of the city. Further investigation is needed to understand cause of failure. This concentration of pipes in poor condition may be because the inspection data are only available for those areas, while data for other parts of the city are not available.

Figure G-6 shows a map of performance index obtained by F-PIE. The result is color coded with green representing excellent pipes and red representing failed pipes. The results show that most of the pipes are rated 0,1 , and 2 . The majority of the poor pipes are concentrated in the center of the city. 


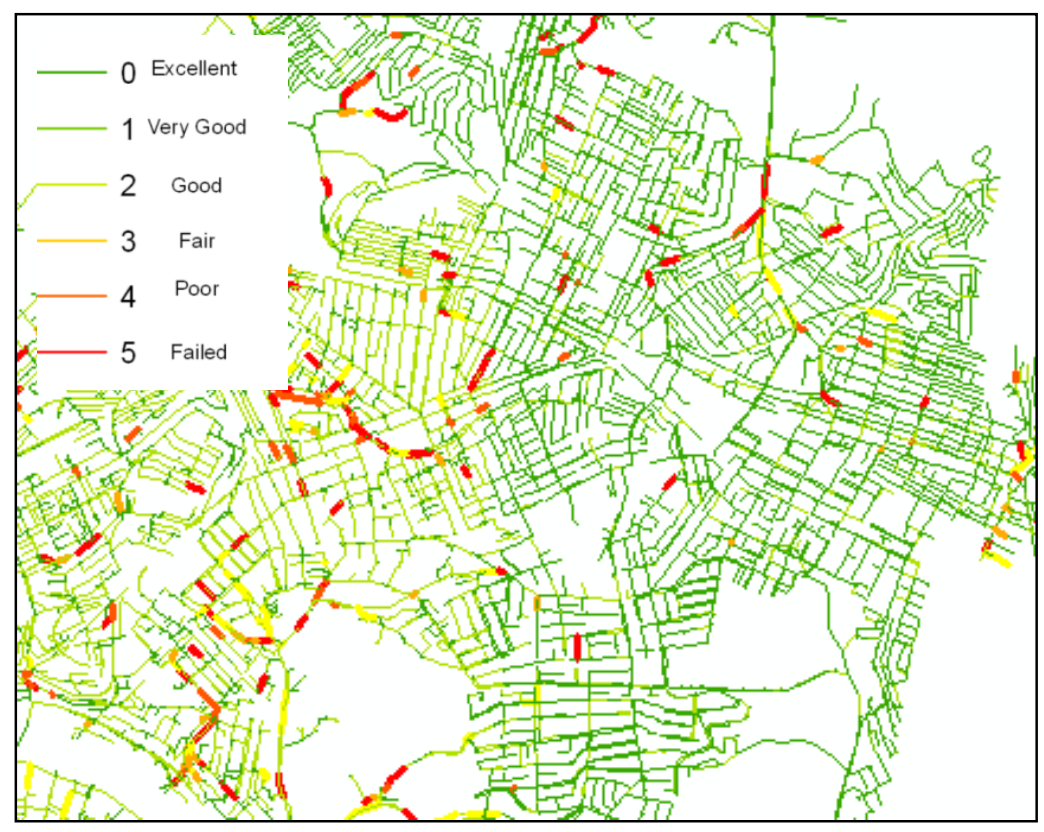

Figure G-5. Performance Index by W-PIE.

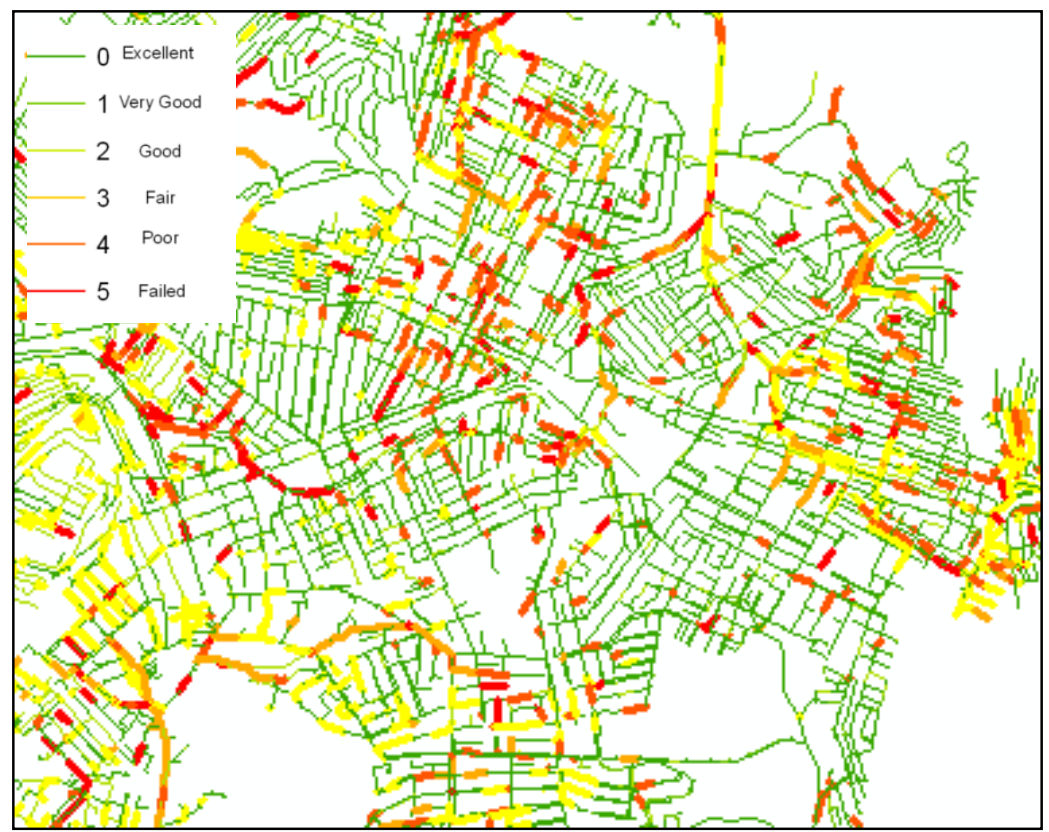

Figure G-6. Performance Index by F-PIE.

From the results presented here, the failed and poor pipes are concentrated in two small areas of the city. Further investigation is needed to understand cause of failure. This concentration of pipes in poor condition may be because the inspection data are only available for those two areas, while data for other parts of the city are not available. 


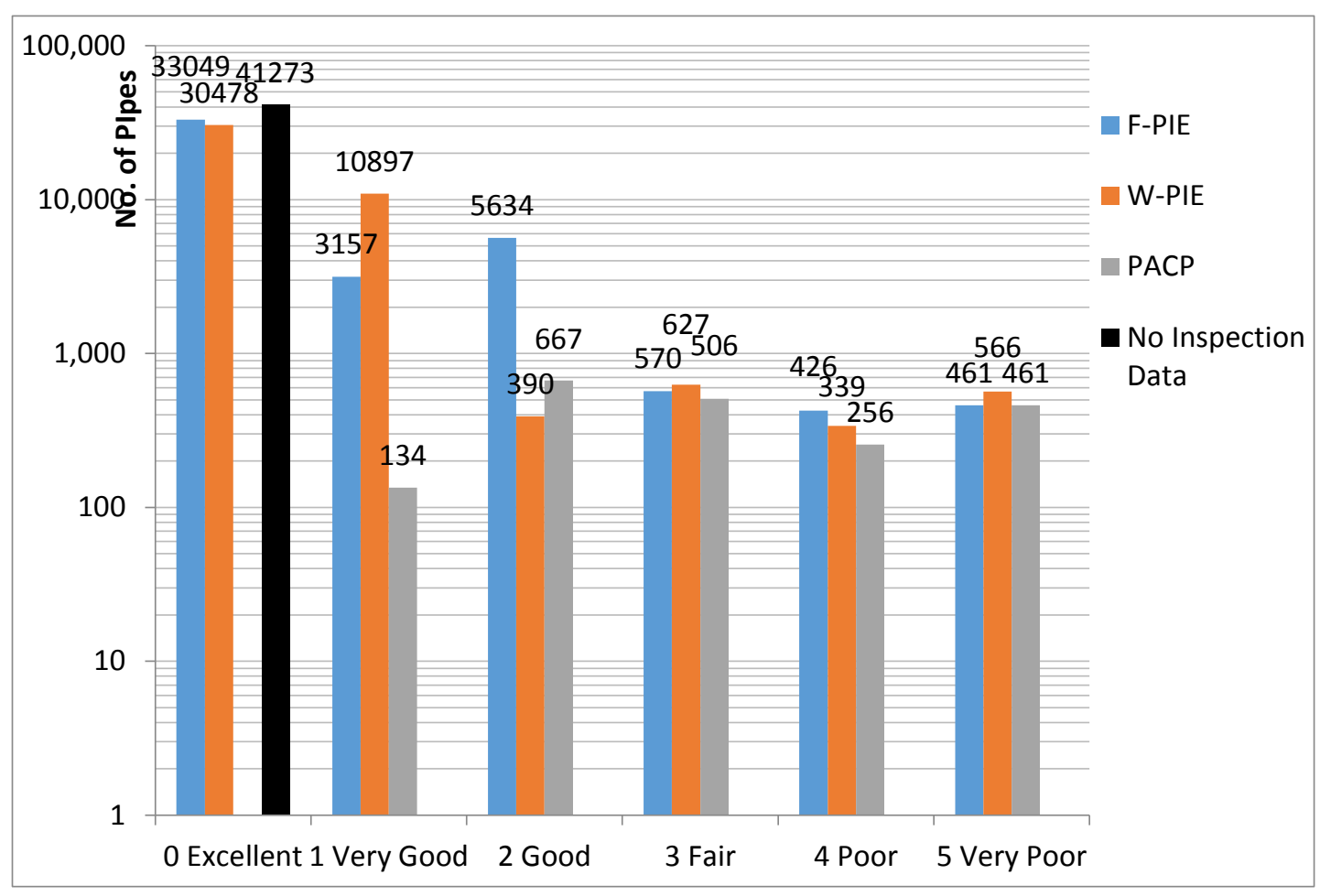

Figure G-7. Performance Index by PACP, F-PIE and W-PIE.

Figure G-7 shows a bar chart of the performance index calculated by No-PACP, PACP, $\mathrm{W}$-PIE and F-PIE illustrating the distribution of the results where the $\mathrm{X}$-axis is the performance index and $y$-axis is the number of pipes that fall within each value. Please note that there are 41,273 pipes that do not have inspection records (represented by No-PACP data) so the higher percentage of excellent may not reflect the actual condition of the pipe in the field.

The results show that most of the pipes are 0,1 , and 2 which are in excellent, very good, and good, respectively. There is little difference in the number of fair (3), and poor (4) pipes in the results provided by F-PIE, W-PIE and, PACP. However, results from F-PIE and W-PIE show more very good and good rated pipes. These results indicate that pipes are shifted from excellent because of the knowledge base. Fair (3), poor (4), and very poor (5) pipes account for approximately 4 percent of entire wastewater pipe system.

\subsection{Result Comparisons}

The results calculated by the weighted factor and fuzzy inference models are compared to PACP rating in Tables G-10, and G-11. Table G-10 shows the results obtained by F-PIE comparing to the PACP rating index where the PACP rating index shows in row, and the results from F-PIE shows in column respectively. Table G-11 shows the performance index calculated from W-PIE compared to the PACP rating index and Table G-12 compares the results of W-PIE and F-PIE. The tables provide similar information as a bar chart above but in greater detail. 
Table G-10. PACP and F-PIE Comparison.

\begin{tabular}{|c|c|c|c|c|c|c|c|c|c|}
\hline & \multicolumn{6}{|c|}{ F_PIE } & \multirow[b]{2}{*}{ Total } \\
\hline & & & 0 & 1 & 2 & 3 & 4 & 5 & \\
\hline \multirow{24}{*}{ PACP } & \multirow{4}{*}{$\begin{array}{l}0 \text { (No PACP } \\
\text { data) }\end{array}$} & No. of pipes & 33049 & 3053 & 5170 & 1 & 0 & 0 & 41273 \\
\hline & & $\%$ within PACP & $80.1 \%$ & $7.4 \%$ & $12.5 \%$ & $.0 \%$ & $.0 \%$ & $.0 \%$ & $100.0 \%$ \\
\hline & & $\%$ within F_PIE & $100.0 \%$ & $96.7 \%$ & $91.8 \%$ & $.2 \%$ & $.0 \%$ & $.0 \%$ & $95.3 \%$ \\
\hline & & $\%$ of Total & $76.3 \%$ & $7.1 \%$ & $11.9 \%$ & $.0 \%$ & $.0 \%$ & $.0 \%$ & $95.3 \%$ \\
\hline & \multirow[t]{4}{*}{1} & No. of pipes & 0 & 104 & 30 & 0 & 0 & 0 & 134 \\
\hline & & $\%$ within PACP & $.0 \%$ & $77.6 \%$ & $22.4 \%$ & $.0 \%$ & $.0 \%$ & $.0 \%$ & $100.0 \%$ \\
\hline & & $\%$ within F_PIE & $.0 \%$ & $3.3 \%$ & $.5 \%$ & $.0 \%$ & $.0 \%$ & $.0 \%$ & $.3 \%$ \\
\hline & & $\%$ of Total & $.0 \%$ & $.2 \%$ & $.1 \%$ & $.0 \%$ & $.0 \%$ & $.0 \%$ & $.3 \%$ \\
\hline & \multirow[t]{4}{*}{2} & No. of pipes & 0 & 0 & 434 & 233 & 0 & 0 & 667 \\
\hline & & $\%$ within PACP & $.0 \%$ & $.0 \%$ & $65.1 \%$ & $34.9 \%$ & $.0 \%$ & $.0 \%$ & $100.0 \%$ \\
\hline & & $\%$ within F_PIE & $.0 \%$ & $.0 \%$ & $7.7 \%$ & $40.9 \%$ & $.0 \%$ & $.0 \%$ & $1.5 \%$ \\
\hline & & $\%$ of Total & $.0 \%$ & $.0 \%$ & $1.0 \%$ & $.5 \%$ & $.0 \%$ & $.0 \%$ & $1.5 \%$ \\
\hline & \multirow[t]{4}{*}{3} & No. of pipes & 0 & 0 & 0 & 336 & 170 & 0 & 506 \\
\hline & & $\%$ within PACP & $.0 \%$ & $.0 \%$ & $.0 \%$ & $66.4 \%$ & $33.6 \%$ & $.0 \%$ & $100.0 \%$ \\
\hline & & $\%$ within F_PIE & $.0 \%$ & $.0 \%$ & $.0 \%$ & $58.9 \%$ & $39.9 \%$ & $.0 \%$ & $1.2 \%$ \\
\hline & & $\%$ of Total & $.0 \%$ & $.0 \%$ & $.0 \%$ & $.8 \%$ & $.4 \%$ & $.0 \%$ & $1.2 \%$ \\
\hline & \multirow[t]{4}{*}{4} & No. of pipes & 0 & 0 & 0 & 0 & 256 & 0 & 256 \\
\hline & & $\%$ within PACP & $.0 \%$ & $.0 \%$ & $.0 \%$ & $.0 \%$ & $100.0 \%$ & $.0 \%$ & $100.0 \%$ \\
\hline & & $\%$ within F_PIE & $.0 \%$ & $.0 \%$ & $.0 \%$ & $.0 \%$ & $60.1 \%$ & $.0 \%$ & $.6 \%$ \\
\hline & & $\%$ of Total & $.0 \%$ & $.0 \%$ & $.0 \%$ & $.0 \%$ & $.6 \%$ & $.0 \%$ & $.6 \%$ \\
\hline & \multirow[t]{4}{*}{5} & No. of pipes & 0 & 0 & 0 & 0 & 0 & 461 & 461 \\
\hline & & $\%$ within PACP & $.0 \%$ & $.0 \%$ & $.0 \%$ & $.0 \%$ & $.0 \%$ & $100.0 \%$ & $100.0 \%$ \\
\hline & & $\%$ within F_PIE & $.0 \%$ & $.0 \%$ & $.0 \%$ & $.0 \%$ & $.0 \%$ & $100.0 \%$ & $1.1 \%$ \\
\hline & & $\%$ of Total & $.0 \%$ & $.0 \%$ & $.0 \%$ & $.0 \%$ & $.0 \%$ & $1.1 \%$ & $1.1 \%$ \\
\hline \multirow{4}{*}{\multicolumn{2}{|c|}{ Total }} & No. of pipes & 33049 & 3157 & 5634 & 570 & 426 & 461 & 43297 \\
\hline & & $\%$ within PACP & $76.3 \%$ & $7.3 \%$ & $13.0 \%$ & $1.3 \%$ & $1.0 \%$ & $1.1 \%$ & $100.0 \%$ \\
\hline & & $\%$ within F_PIE & $100.0 \%$ & $100.0 \%$ & $100.0 \%$ & $100.0 \%$ & $100.0 \%$ & $100.0 \%$ & $100.0 \%$ \\
\hline & & $\%$ of Total & $76.3 \%$ & $7.3 \%$ & $13.0 \%$ & $1.3 \%$ & $1.0 \%$ & $1.1 \%$ & $100.0 \%$ \\
\hline
\end{tabular}

*\% within PACP $=\frac{\text { Number of pipes }}{\text { Row } \text { Total }(P A C P)} \times 100$

$* * \%$ within $F-P I E=\frac{\text { Number of pipes }}{\text { Column Total }(F-P I E)} \times 100$

$* * * \%$ of Total $=\frac{\text { Number of pipes }}{\text { Total }} \times 100$ 
Table G-11. PACP and W-PIE Comparison.

\begin{tabular}{|c|c|c|c|c|c|c|c|c|c|}
\hline & \multicolumn{6}{|c|}{ W_PIE } & \multirow[b]{2}{*}{ Total } \\
\hline & & & 0 & 1 & 2 & 3 & 4 & 5 & \\
\hline \multirow[t]{24}{*}{ PACP } & \multirow{4}{*}{$\begin{array}{l}.0 \text { ( No PACP } \\
\text { data) }\end{array}$} & No. of pipes & 30478 & 10795 & 0 & 0 & 0 & 0 & 41273 \\
\hline & & $\%$ within PACP & $73.8 \%$ & $26.2 \%$ & $.0 \%$ & $.0 \%$ & $.0 \%$ & $.0 \%$ & $100.0 \%$ \\
\hline & & $\%$ within W_PIE & $100.0 \%$ & $99.1 \%$ & $.0 \%$ & $.0 \%$ & $.0 \%$ & $.0 \%$ & $95.3 \%$ \\
\hline & & $\%$ of Total & $70.4 \%$ & $24.9 \%$ & $.0 \%$ & $.0 \%$ & $.0 \%$ & $.0 \%$ & $95.3 \%$ \\
\hline & \multirow[t]{4}{*}{1} & No. of pipes & 0 & 102 & 32 & 0 & 0 & 0 & 134 \\
\hline & & $\%$ within PACP & $.0 \%$ & $76.1 \%$ & $23.9 \%$ & $.0 \%$ & $.0 \%$ & $.0 \%$ & $100.0 \%$ \\
\hline & & \% within W_PIE & $.0 \%$ & $.9 \%$ & $8.2 \%$ & $.0 \%$ & $.0 \%$ & $.0 \%$ & $.3 \%$ \\
\hline & & $\%$ of Total & $.0 \%$ & $.2 \%$ & $.1 \%$ & $.0 \%$ & $.0 \%$ & $.0 \%$ & $.3 \%$ \\
\hline & \multirow[t]{4}{*}{2} & No. of pipes & 0 & 0 & 358 & 309 & 0 & 0 & 667 \\
\hline & & $\%$ within PACP & $.0 \%$ & $.0 \%$ & $53.7 \%$ & $46.3 \%$ & $.0 \%$ & $.0 \%$ & $100.0 \%$ \\
\hline & & $\%$ within W_PIE & $.0 \%$ & $.0 \%$ & $91.8 \%$ & $49.3 \%$ & $.0 \%$ & $.0 \%$ & $1.5 \%$ \\
\hline & & $\%$ of Total & $.0 \%$ & $.0 \%$ & $.8 \%$ & $.7 \%$ & $.0 \%$ & $.0 \%$ & $1.5 \%$ \\
\hline & \multirow[t]{4}{*}{3} & No. of pipes & 0 & 0 & 0 & 318 & 188 & 0 & 506 \\
\hline & & $\%$ within PACP & $.0 \%$ & $.0 \%$ & $.0 \%$ & $62.8 \%$ & $37.2 \%$ & $.0 \%$ & $100.0 \%$ \\
\hline & & \% within W_PIE & $.0 \%$ & $.0 \%$ & $.0 \%$ & $50.7 \%$ & $55.5 \%$ & $.0 \%$ & $1.2 \%$ \\
\hline & & $\%$ of Total & $.0 \%$ & $.0 \%$ & $.0 \%$ & $.7 \%$ & $.4 \%$ & $.0 \%$ & $1.2 \%$ \\
\hline & \multirow[t]{4}{*}{4} & No. of pipes & 0 & 0 & 0 & 0 & 151 & 105 & 256 \\
\hline & & $\%$ within PACP & $.0 \%$ & $.0 \%$ & $.0 \%$ & $.0 \%$ & $59.0 \%$ & $41.0 \%$ & $100.0 \%$ \\
\hline & & \% within W_PIE & $.0 \%$ & $.0 \%$ & $.0 \%$ & $.0 \%$ & $44.5 \%$ & $18.6 \%$ & $.6 \%$ \\
\hline & & $\%$ of Total & $.0 \%$ & $.0 \%$ & $.0 \%$ & $.0 \%$ & $.3 \%$ & $.2 \%$ & $.6 \%$ \\
\hline & \multirow[t]{4}{*}{5} & No. of pipes & 0 & 0 & 0 & 0 & 0 & 461 & 461 \\
\hline & & $\%$ within PACP & $.0 \%$ & $.0 \%$ & $.0 \%$ & $.0 \%$ & $.0 \%$ & $100.0 \%$ & $100.0 \%$ \\
\hline & & \% within W_PIE & $.0 \%$ & $.0 \%$ & $.0 \%$ & $.0 \%$ & $.0 \%$ & $81.4 \%$ & $1.1 \%$ \\
\hline & & $\%$ of Total & $.0 \%$ & $.0 \%$ & $.0 \%$ & $.0 \%$ & $.0 \%$ & $1.1 \%$ & $1.1 \%$ \\
\hline \multirow[t]{4}{*}{ Total } & & No. of pipes & 30478 & 10897 & 390 & 627 & 339 & 566 & 43297 \\
\hline & & $\%$ within PACP & $70.4 \%$ & $25.2 \%$ & $.9 \%$ & $1.4 \%$ & $.8 \%$ & $1.3 \%$ & $100.0 \%$ \\
\hline & & \% within W_PIE & $100.0 \%$ & $100.0 \%$ & $100.0 \%$ & $100.0 \%$ & $100.0 \%$ & $100.0 \%$ & $100.0 \%$ \\
\hline & & $\%$ of Total & $70.4 \%$ & $25.2 \%$ & $.9 \%$ & $1.4 \%$ & $.8 \%$ & $1.3 \%$ & $100.0 \%$ \\
\hline
\end{tabular}

* \% within PACP $=\frac{\text { Number of pipes }}{\text { Row Total }(P A C P)} \times 100$

** \% within $W-P I E=\frac{\text { Number of pipes }}{\text { Column Total }(W-P I E)} \times 100$

$* * * \%$ of Total $=\frac{\text { Number of pipes }}{\text { Total }} \times 100$ 
Table G-12. W-PIE and F-PIE Comparison.

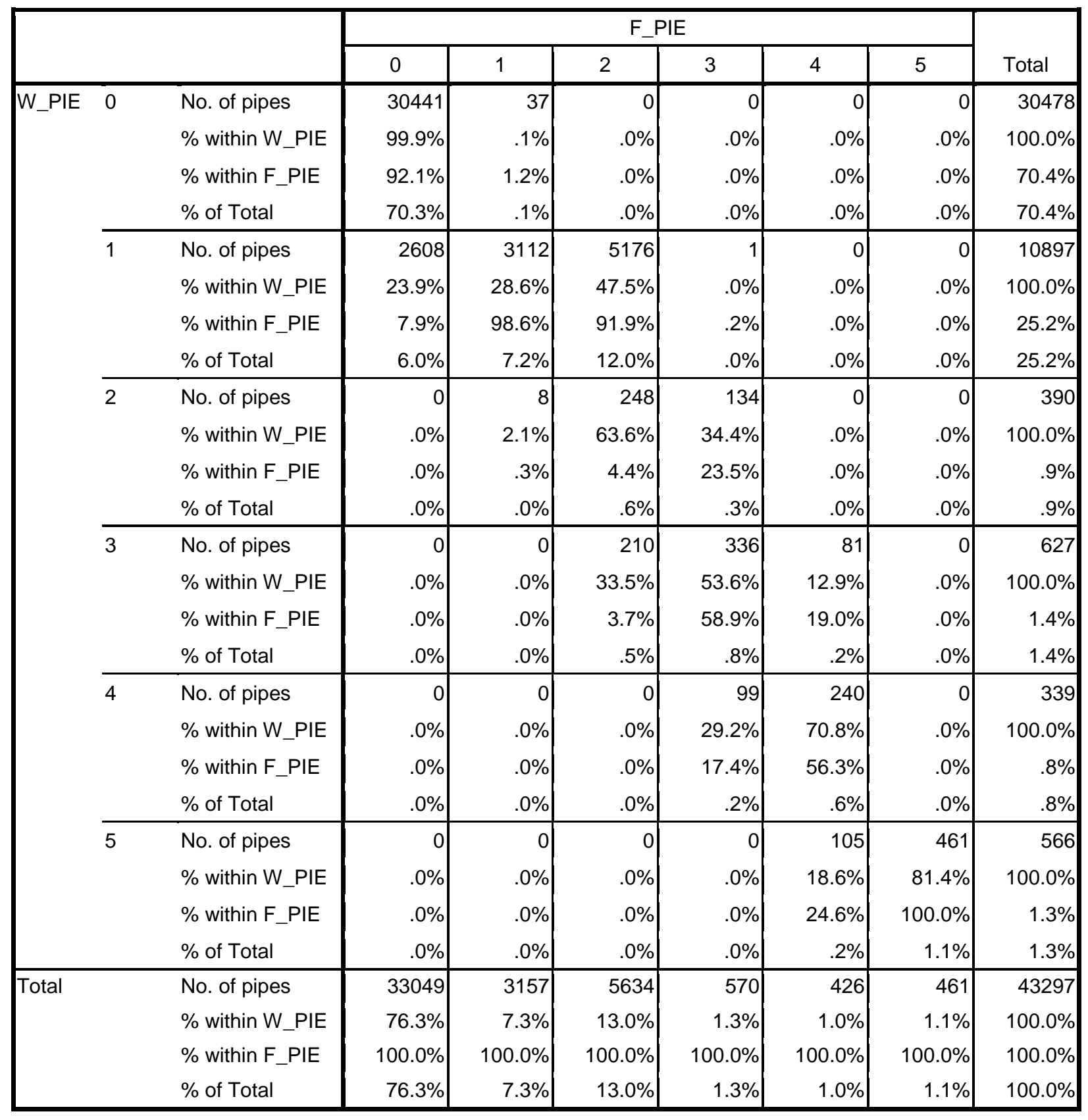

$* \%$ within $W-P I E=\frac{\text { Number of pipes }}{\text { Row } \operatorname{Total}(W-P I E)} \times 100$

** \% within $F-P I E=\frac{\text { Number of pipes }}{\text { Column Total }(F-P I E)} \times 100$

$* * * \%$ of Total $=\frac{\text { Number of pipes }}{\text { Total }} \times 100$ 


\section{City $\mathrm{F}$}

We analyzed data received from the City F and evaluated each pipe section (manhole to manhole) using performance models (weighted factor and fuzzy inference system). The table mainly consists of City F's inspection and maintenance records and general sewer main data. More detail about the data supplied by City F can be found in appendix F. We were provided with the data including seven parameters used in this pilot study.

\section{Parameters}
a. Pipe age
b. Pipe diameter
c. Pipe depth
d. Pipe slope
e. Pipe length
f. Pipe material
g. Location
h. Pipe condition (inspection records)

The parameters listed above are stored as attributes in mainline table. The City F's inhouse rating system ranks the wastewater pipes in $\mathrm{A}, \mathrm{B}$ and $\mathrm{C}$ ratings.

The designation A is considered as good condition pipes and no repair required. Defects that may be seen in pipes rated A are hairline cracks, minor infiltration and rust deposits, for example. $\mathrm{B}$ is considered damaged pipes and re-inspect within 3 years are required. . Defects that may be seen in the $\mathrm{B}$ are minor corrosion, infiltration, leakage at joints, and cracking. $\mathrm{C}$ is considered severe damaged pipes and feasibility study is needed. Defects that may be seen in the $\mathrm{C}$ are severe corrosion, high infiltration, missing brick or mortar, lateral cracks, and broken. Pipe condition was converted from in-house to zero to five scales for evaluation purpose, where A, B, and $\mathrm{C}$ were converted to 1,2 , and 4 respectively. Based on limited data the performance models produced the results indicated in Figure G-8 below.

Figure G-8 is a bar chart of the performance index calculated by in the in-house rating index, W-PIE, and F-PIE. The x-axis is the performance index and y-axis is the number of pipes that fall within each value. The pipes that do not have inspection data, indicated in black, are assumed excellent for the purpose of calculation. The results are widely distributed. Pipes that inhouse rates "A", F-PIE rates them a 1 (very good), 2 (good), or 3 (fair). Pipes that in-house rates "B", F-PIE rates them a 2 (good), or 3 (fair). Pipes that in-house rates "C", F-PIE rates them a 4 (poor).

The results from W-PIE shows only one step different from in-house rating, for example if in-house rates "A", W-PIE rates 2 (good), or 3 (fair). 


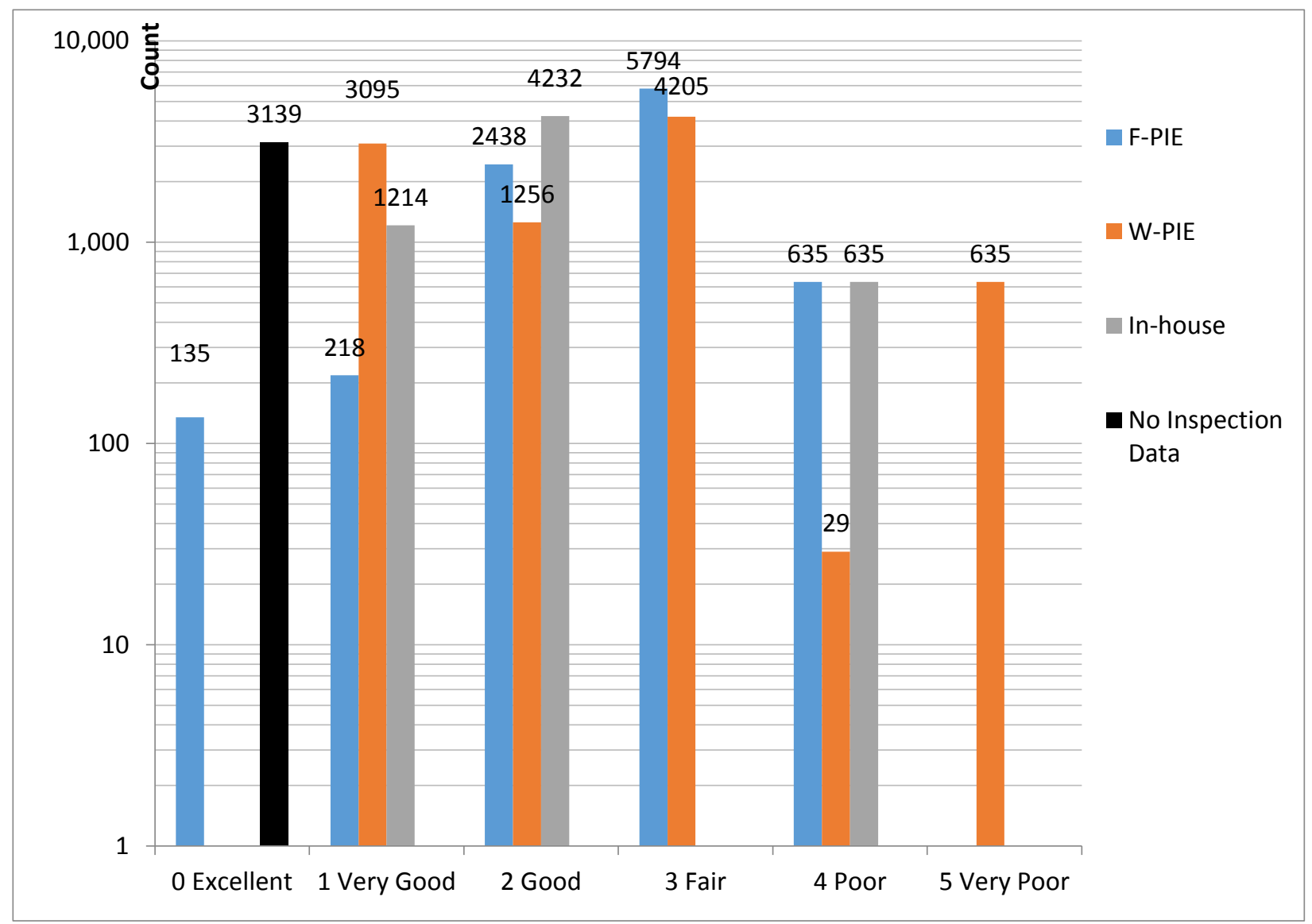

Figure G-8. Performance Index by In-House, F-PIE and W-PIE.

\subsection{Result Comparisons}

The results calculated by W-PIE and F-PIE are compared to in-house rating provided by MWRA in Tables G-13, and G-14 respectively and the comparison of results from the weighted factor and fuzzy inference models is shown in Table G-15. Table G-13 shows the comparison between the F-PIE and in-house rating index, where the in-house rating is shown in row and the results from F-PIE is shown in column. The tables provide similar information as a bar chart above but in more detail. 
Table G-13. In-House Rating Index and F-PIE Comparison

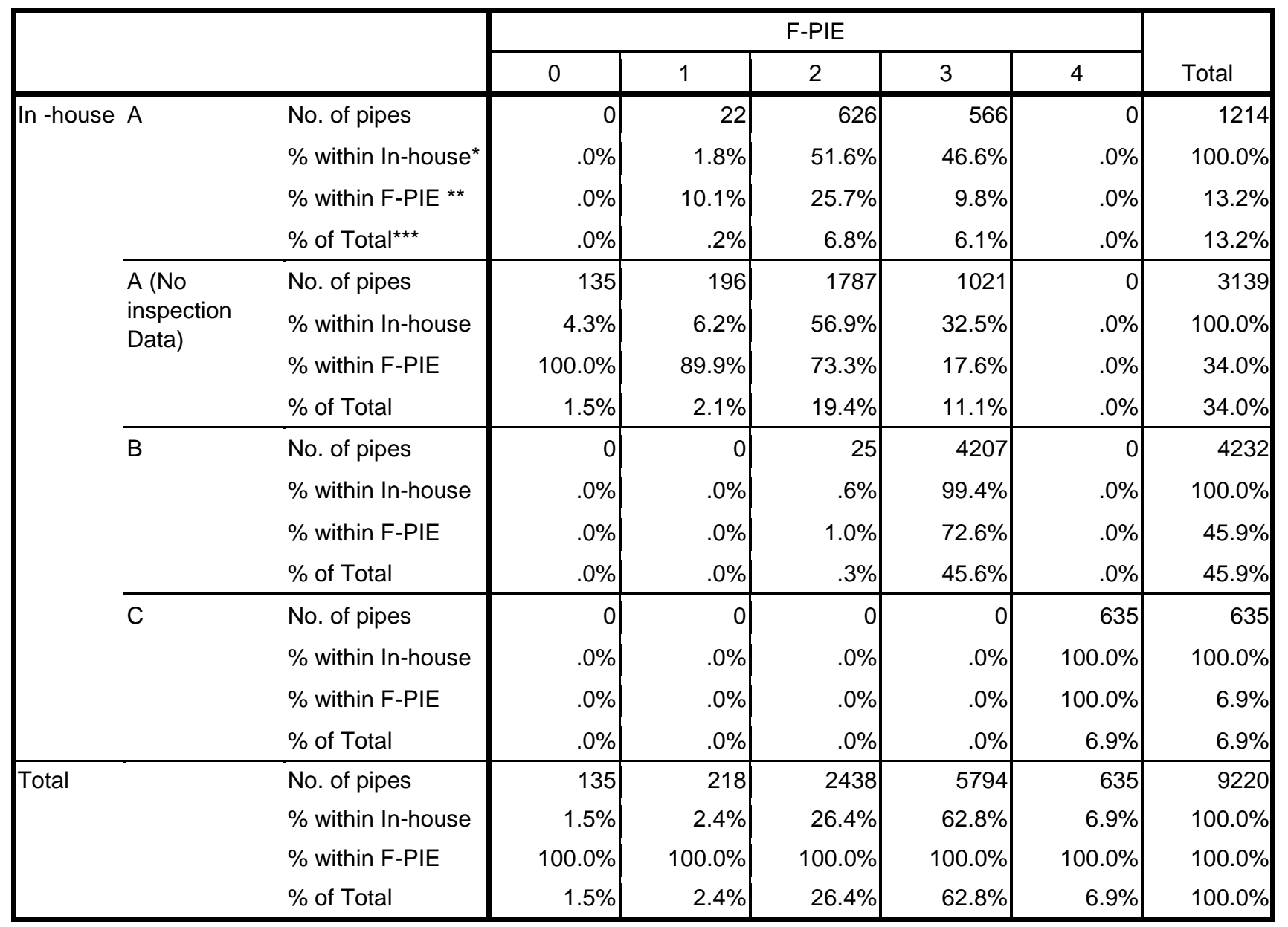

$* \%$ within In - house $=\frac{\text { Number of pipes }}{\text { Row } \operatorname{Total}(\text { In-house })} \times 100$

** \% within $F-P I E=\frac{\text { Number of pipes }}{\text { Column Total }(F-P I E)} \times 100$

$* * * \%$ of Total $=\frac{\text { Number of pipes }}{\text { Total }} \times 100$ 
Table G-14. In-House Rating Index and W-PIE Comparison

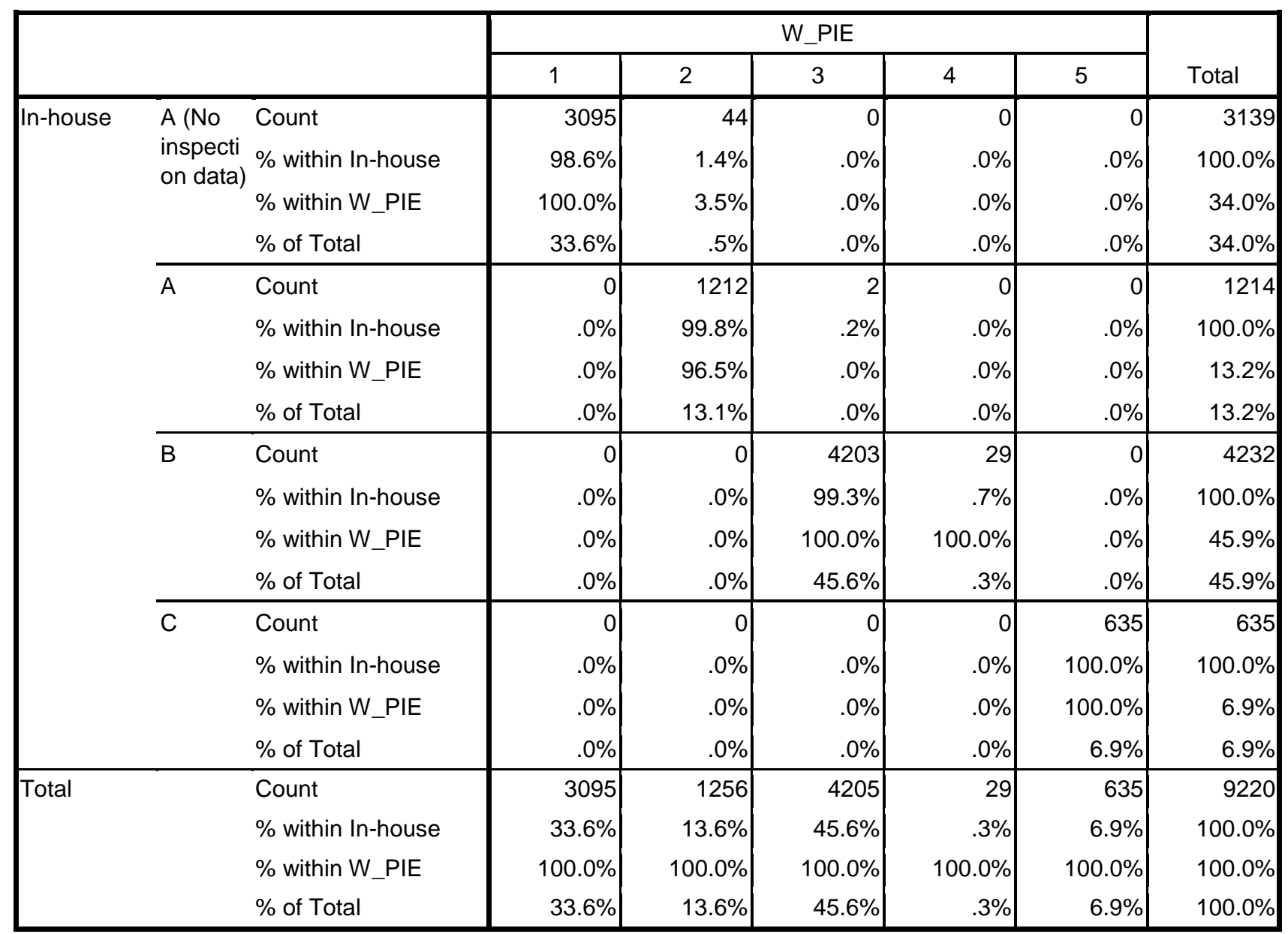

*\% within PACP $=\frac{\text { Number of pipes }}{\text { Row } \text { Total }(\text { In-house })} \times 100$

** $\%$ within $W-P I E=\frac{\text { Number of pipes }}{\text { Column Total }(W-P I E)} \times 100$

$* * * \%$ of Total $=\frac{\text { Number of pipes }}{\text { Total }} \times 100$ 
Table G-15. W-PIE and F-PIE Comparison

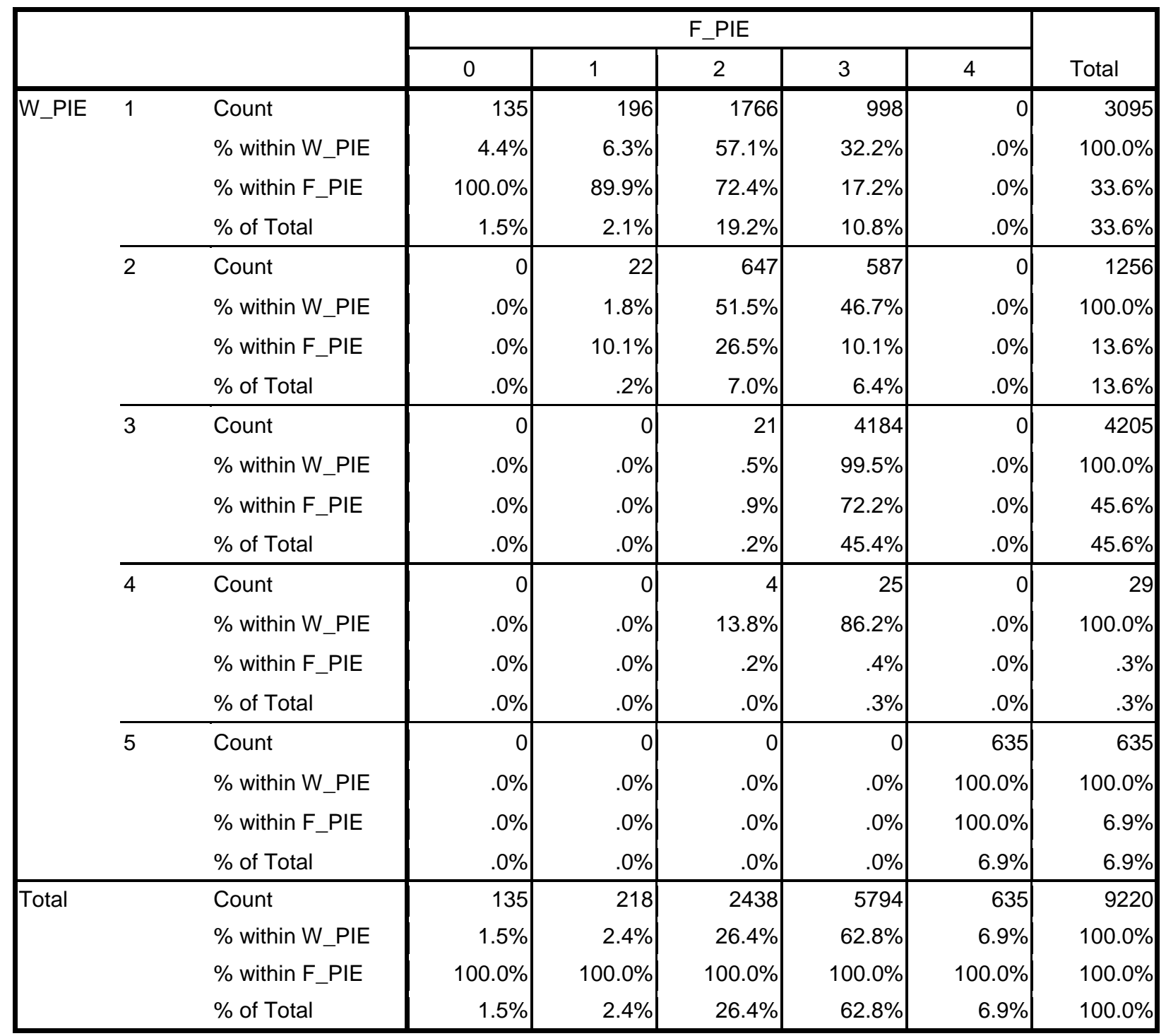

*\% within $W-P I E=\frac{\text { Number of pipes }}{\text { Row } \operatorname{Total}(W-P I E)} \times 100$

$* * \%$ within $F-P I E=\frac{\text { Number of pipes }}{\text { Column } \operatorname{Total}(F-P I E)} \times 100$

*** \% of Total $=\frac{\text { Number of pipes }}{\text { Total }} \times 100$ 


\subsection{Sample Calculations}

The Performance Index was calculated for various pipe sections (manhole to manhole). These sample pipes are from the City F's data. This exercise was conducted to understand the benefits and shortcomings of the performance model. For demonstration of the performance index, some parameters were considered from the utility data however, some parameters were derived and may not be accurate, if the actual data is not available.

\section{Sample 1: Pipe ID 8}

The pipe ID 8 is selected for sample calculations. Details of the pipe are given in Table G-16.

Table G-16. Sample 1 Pipe Data.

\begin{tabular}{|l|l|l|l|}
\hline No. & Parameter & Unit & Value \\
\hline 1 & Pipe Age & Year & 114 \\
\hline 2 & Pipe Depth & Feet & 20 \\
\hline 3 & Pipe Diameter & Inch & 792 \\
\hline 4 & Pipe Length & Feet & 33 \\
\hline 5 & Pipe Slope & Percent & 17 \\
\hline 6 & Location & Feet & 10 \\
\hline 7 & Pipe Condition & Grade & B \\
\hline
\end{tabular}

* Parameters indicated in red are unfavorable parameters

\section{In-house Rating Index:}

The value of the pipe rating by in-house is " $\mathrm{B}$ (fair)". This value is considered average.

\section{F-PIE Index:}

The Performance Index of this pipe had a value of " 3 ". This implies that the pipe is damaged but it is not in the danger of failure. The parameters indicated in red are unfavorable in the performance index.

\section{W-PIE Index:}

Same as F-PIE, the Performance Index of this pipe had a value of "3". This implies that the pipe is damaged but it is not in the danger of failure. The parameters indicated in red are unfavorable in the performance index. 


\section{Index Comparisons: Fair, Fair, Fair}

The in-house rating index of this pipe, ID 8, indicates that the pipe is in average condition with the value of " $\mathrm{B}$ ". The performance indexes give value ' 3 ' consistent with in-house rating. It is also indicating poor performance due to an old pipe age, which may lead to structure failure.

\section{Sample 2: Pipe 247}

The pipe ID 247 is selected for sample calculations. Details of this pipe are given in Table G-17.

Table G-17. Sample 2 Pipe Data.

\begin{tabular}{|l|l|l|l|}
\hline No. & Parameter & Unit & Value \\
\hline 1 & Pipe Age & Year & 1 \\
\hline 2 & Pipe Depth & Feet & 20 \\
\hline 3 & Pipe Diameter & Inch & 5610 \\
\hline 4 & Pipe Length & Feet & 187 \\
\hline 5 & Pipe Slope & Percent & 18 \\
\hline 5 & Location & Feet & 10 \\
\hline 7 & Pipe Condition & Grade & A (no data) \\
\hline
\end{tabular}

* Parameters indicated in red are unfavorable parameters

\section{In-house Rating Index:}

The value of the pipe rating by in-house is " $\mathrm{A}$ ". There is no inspection data for this pipe.

\section{F-PIE Index:}

The Performance Index of this pipe had a value of " 0 (Excellent)". The parameters indicated in red are unfavorable in the performance index.

\section{W-PIE Index:}

The Performance Index of this pipe had a value of "1 (very good)". The parameters indicated in red are unfavorable to the performance index.

\section{Index Comparisons: No data \& Excellent, Very Good}

The In-house rating index of this pipe, ID 247, indicates that the pipe is in an excellent condition with the value of "A". Even though, there are two parameters that are unfavorable, the value is not high enough to make F-PIE indicate that as a cause of failure. The performance indexes gives value " 0 " and " 1 " to this pipe section consistent with the in-house rating index "A". 


\section{City G}

The City G gave two shape-files and one geo-database table. The shape-files consist of the main pipelines, manholes, and a sewershed geo-database table. The sewer main shape file consists of all the sewer mains within the City G town limits which are spatially mapped in the projected coordinate system. The sewer main attributes table consists of various pipe parameters such as Pipe ID, diameter, material, section length, joint type, street address, connected manhole, manhole condition, and pipe condition. Also, the length of the pipe was calculated and added as an attribute. Although a few columns were left blank, information was collected for around 4,700 pipes. More detail about the data supplied by City G can be found in appendix F.

There were five parameters that used in this study.

\section{Parameters}
a. Pipe diameter
b. Pipe length
c. Pipe material
d. Soil type
e. Pipe condition (Condition Rating)

The parameters listed above are stored in mainline table and can be directly employed in the model. Pipe condition data provided by City G are coded as good, average and bad. It was transformed to $0-5$ based-condition scale before analyzing.

\subsection{W-PIE and F-PIE Results}

Figure G-9 shows a bar chart of the performance index calculated by In-house, W-PIE, and F-PIE and the x-axis is the performance index and y-axis is the number of pipes that fall within each value. There are only about 130 pipe sections that have inspection data, so it was assumed that the rest of the pipes are in excellent condition, indicated in black.

The results show that most of the pipes are 0 , and 1 which are in excellent and very good, respectively. There is little difference in the number of fair (3), and poor (4) pipes in the results provided by F-PIE, W-PIE and, PACP. However, results from F-PIE and W-PIE show more pipes rated very good and good which indicates these pipes are shifted from excellent pipes because of the knowledge base. Fair (3), poor (4), and very poor (5) rated pipes account for approximately 8 percent of the entire wastewater pipe system.

\subsection{Result Comparisons}

The results calculated by the weighted factor and fuzzy inference models are compared to PACP rating in Tables G-18, and G-19. Table G-18 shows the results obtained by F-PIE compared to the PACP rating index (shown in rows), and the results from F-PIE shown in columns. Table G-19 shows the performance index calculated from W-PIE compared to the 
PACP rating index. Table G-20 shows the comparison of the results between W-PIE and F-PIE. The tables provide similar information as a bar chart above but in more detail.

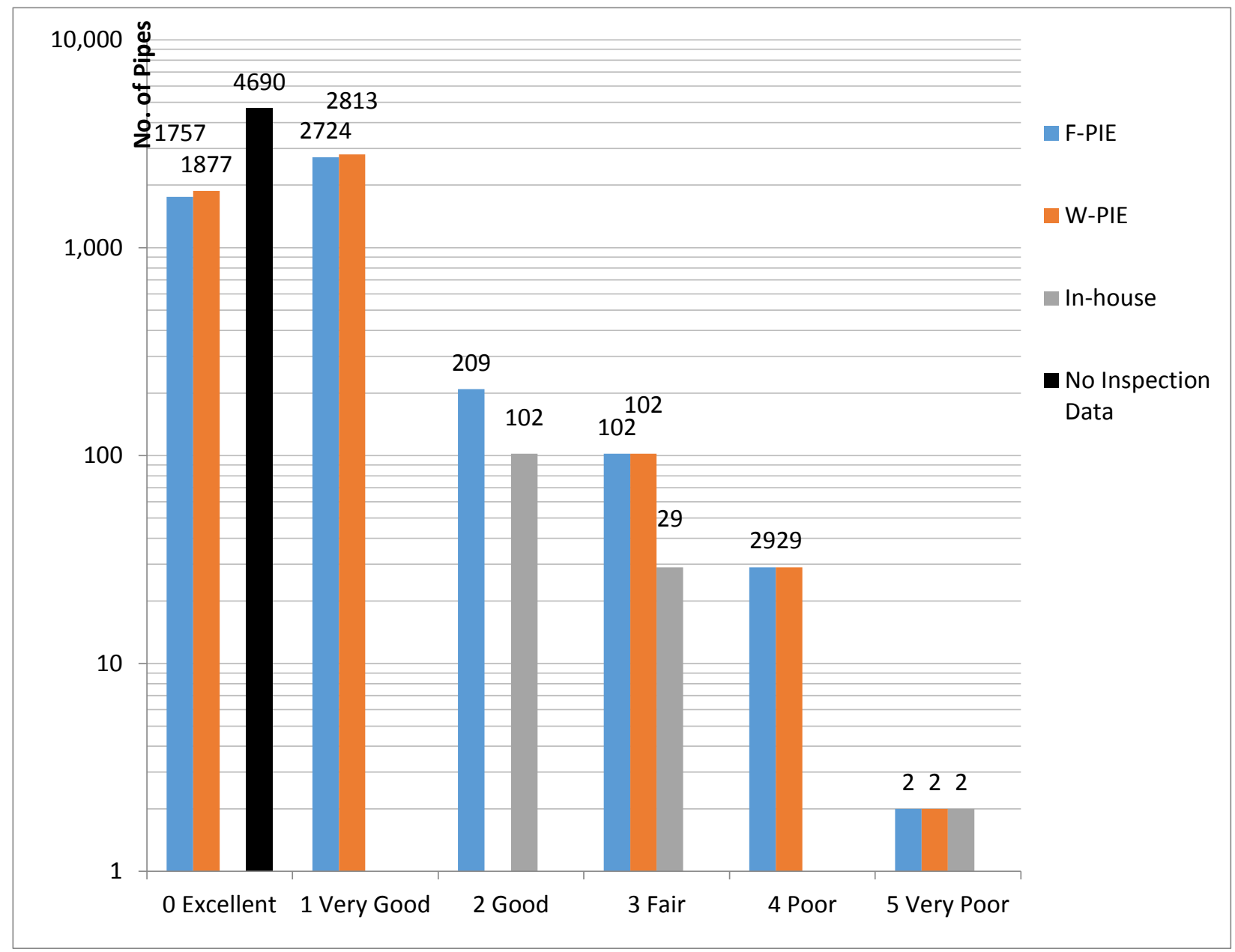

Figure G-9. Performance Index by In-House Rating System, W-PIE and F-PIE.

The results calculated by W-PIE and F-PIE are compared to In-house rating provided by TBU in Table G-18, and G-19 respectively and the comparison between the results from the weighted factor and fuzzy inference models is shown in Table G-20. The results presented here suggest that there are no significant differences between the three methods. Since there are only a few parameters available, W-PIE and F-PIE evaluate the pipes on only condition, soil type, pipe length, and diameter. Pipe condition has the greatest weighting, of the four parameters, thus the results are similar to in-house rating which used condition in the F-PIE and W-PIE. 
Table G-18. In-House Rating System and F-PIE Comparison.

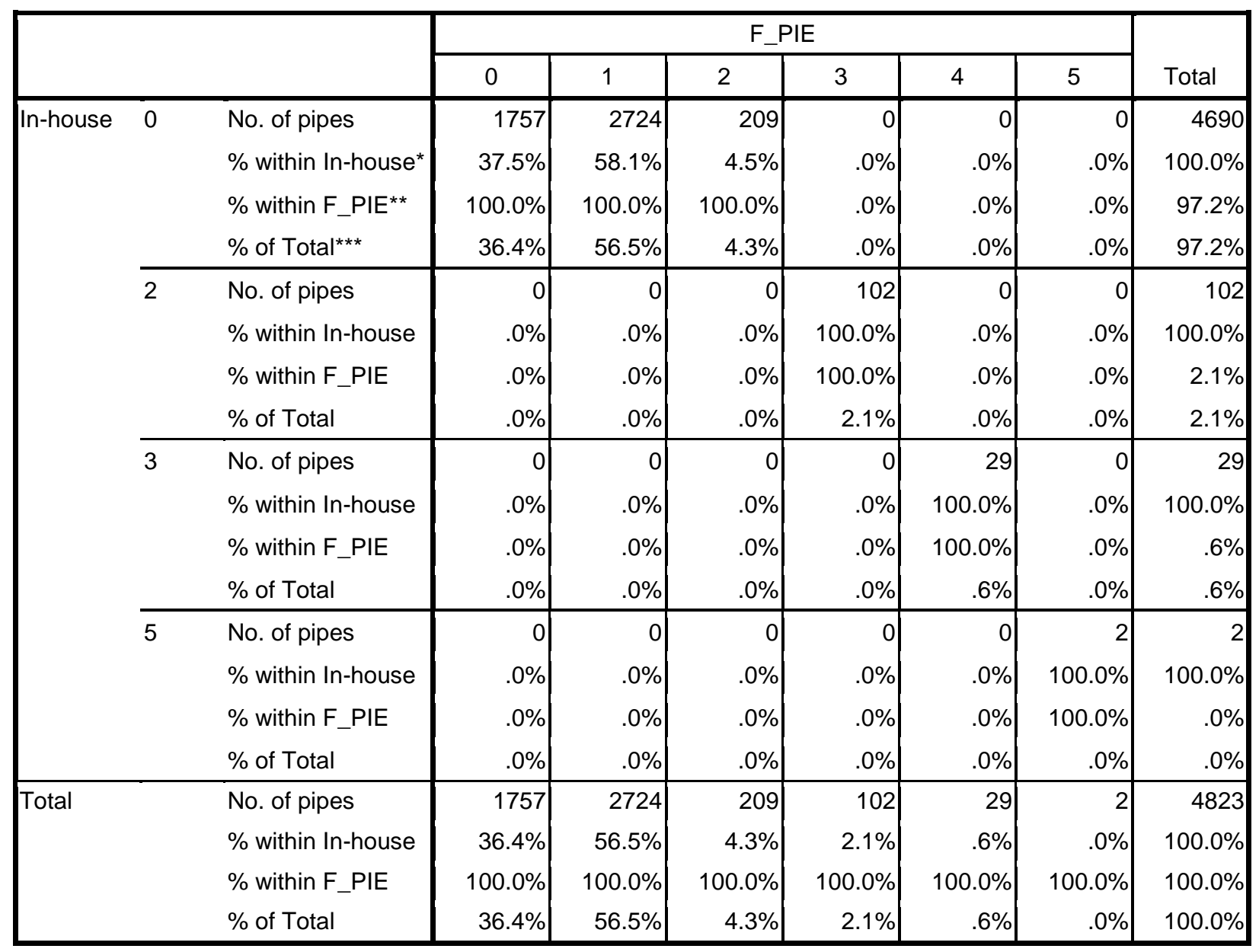

$* \%$ within In - house $=\frac{\text { Number of pipes }}{\text { Row Total }(\text { In-house })} \times 100$

$* * \%$ within $F-P I E=\frac{\text { Number of pipes }}{\text { Column Total }(F-P I E)} \times 100$

*** \% of Total $=\frac{\text { Number of pipes }}{\text { Total }} \times 100$ 
Table G-19. In-house rating and W-PIE Comparison

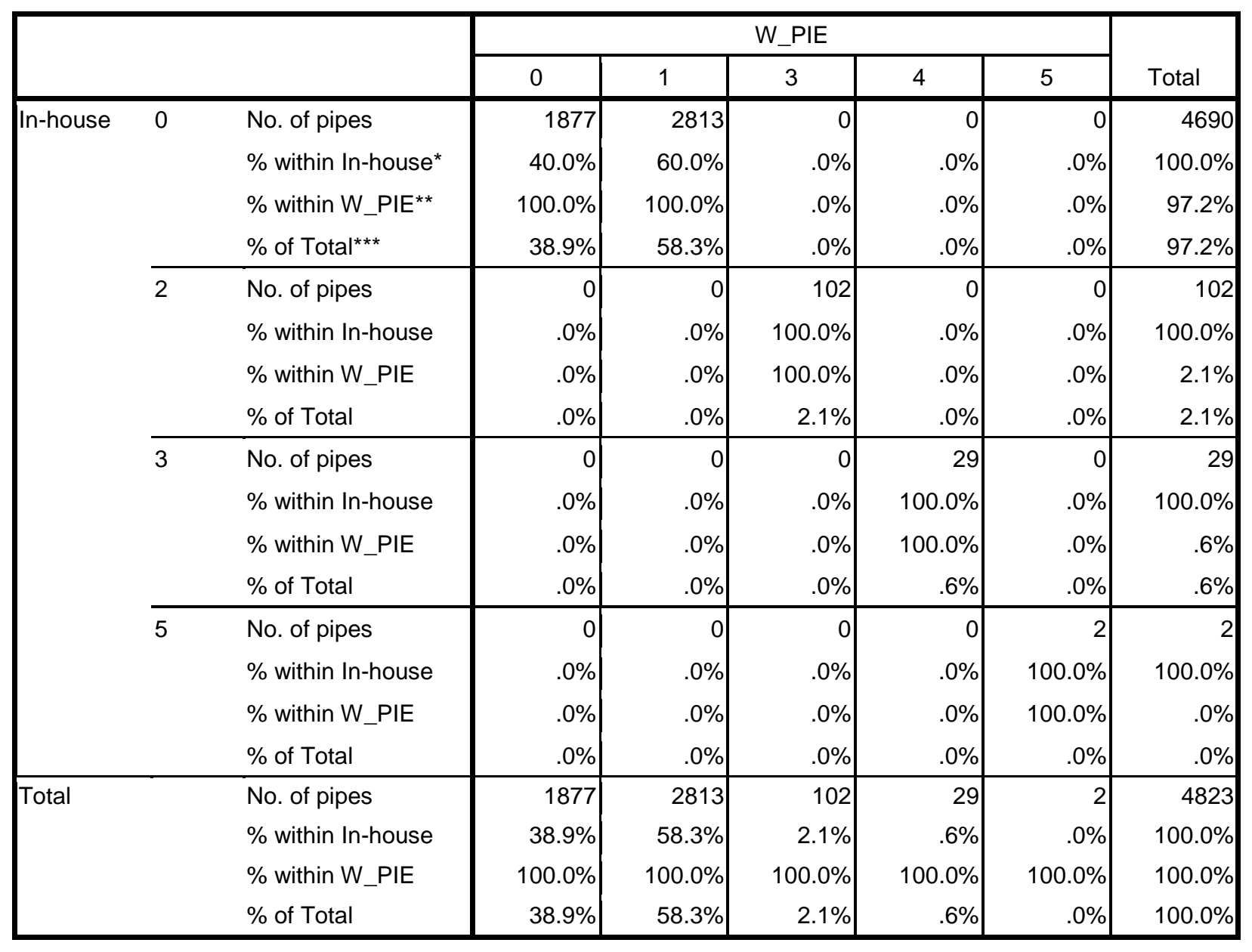

$* \%$ within In - house $=\frac{\text { Number of pipes }}{\text { Row Total }(\text { In-house })} \times 100$

** \% within $W-P I E=\frac{\text { Number of pipes }}{\text { Column Total }(W-P I E)} \times 100$

$* * * \%$ of Total $=\frac{\text { Number of pipes }}{\text { Total }} \times 100$ 
Table G-20. W-PIE and F-PIE Comparison.

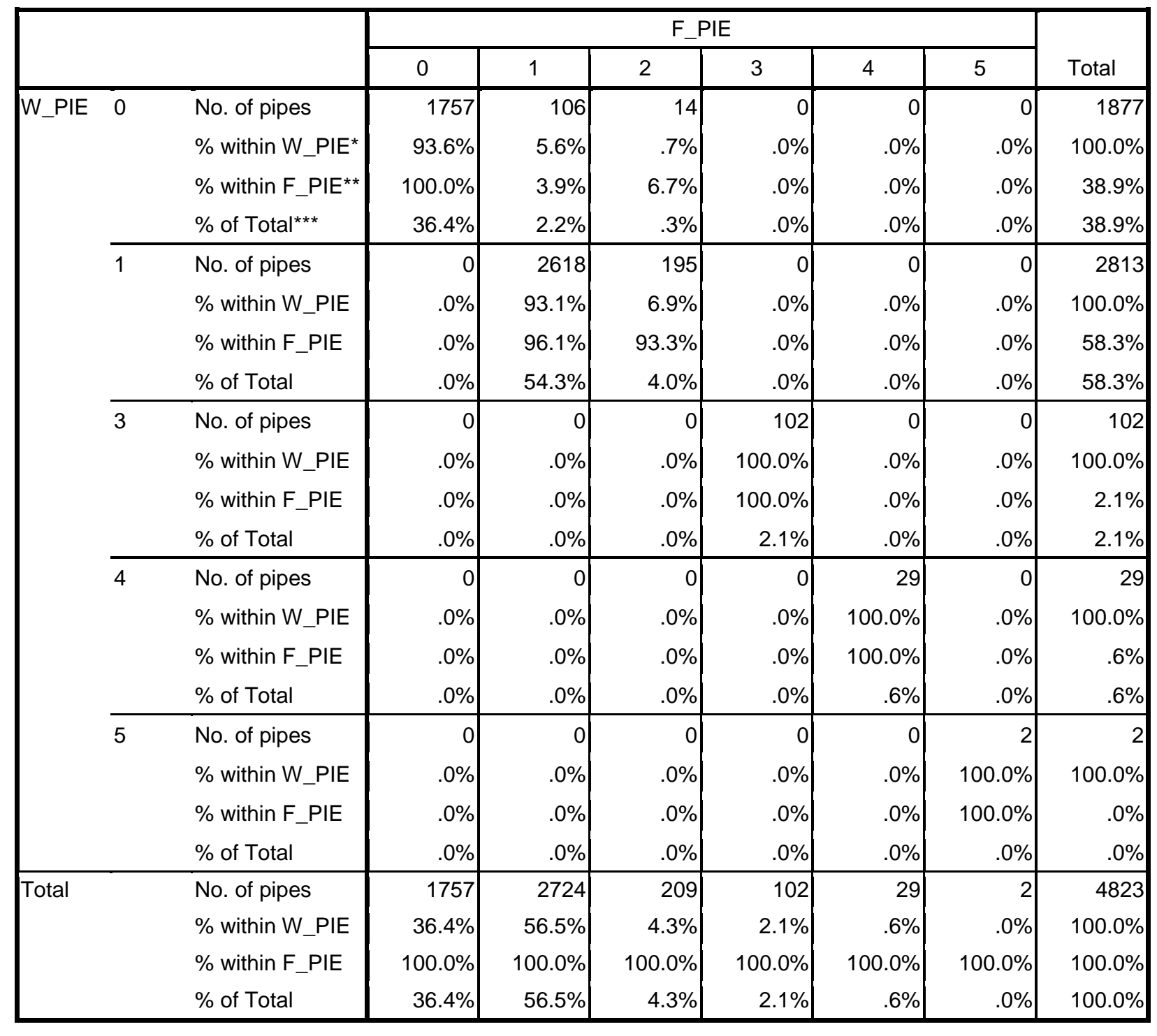

$* \%$ within $W-P I E=\frac{\text { Number of pipes }}{\text { Row } \operatorname{Total}(W-P I E)} \times 100$

** \% within $F-P I E=\frac{\text { Number of pipes }}{\text { Column Total }(F-P I E)} \times 100$

$* * * \%$ of Total $=\frac{\text { Number of pipes }}{\text { Total }} \times 100$ 


\section{City I}

We analyzed data received from City I and evaluated each pipe section (manhole to manhole) using performance models (W-PIE and F-PIE). CCWS sent DVD contains its database to the research group at Virginia Tech. Altogether there were four main geo-database shape-files which consist of City I sewer collection, force main. More detail about the data supplied by City I can be found in appendix F. City I is in an early stage of developing its wastewater pipe database so unfortunately we were provided with the data including only three parameters that can be used in this pilot study.

\section{Parameters}
a. Pipe material
b. Pipe diameter
c. Pipe length

The parameters listed above are stored as attributes in mainline shape-files. City I are in the process of developing its wastewater pipe database; so there was not much data available at the time. Based on limited data we had, we run the performance models with the results presenting below.

\subsection{W-PIE and F-PIE Results}

Figure G-10 shows a bar chart of the performance index calculated by W-PIE and F-PIE to illustrate the distribution of the results, which on $\mathrm{x}$-axis is the performance index and $\mathrm{y}$-axis is the number of pipes that fall within each value. The pipes that do not have inspection data, indicated in black, are assumed excellent for the purpose of calculation.

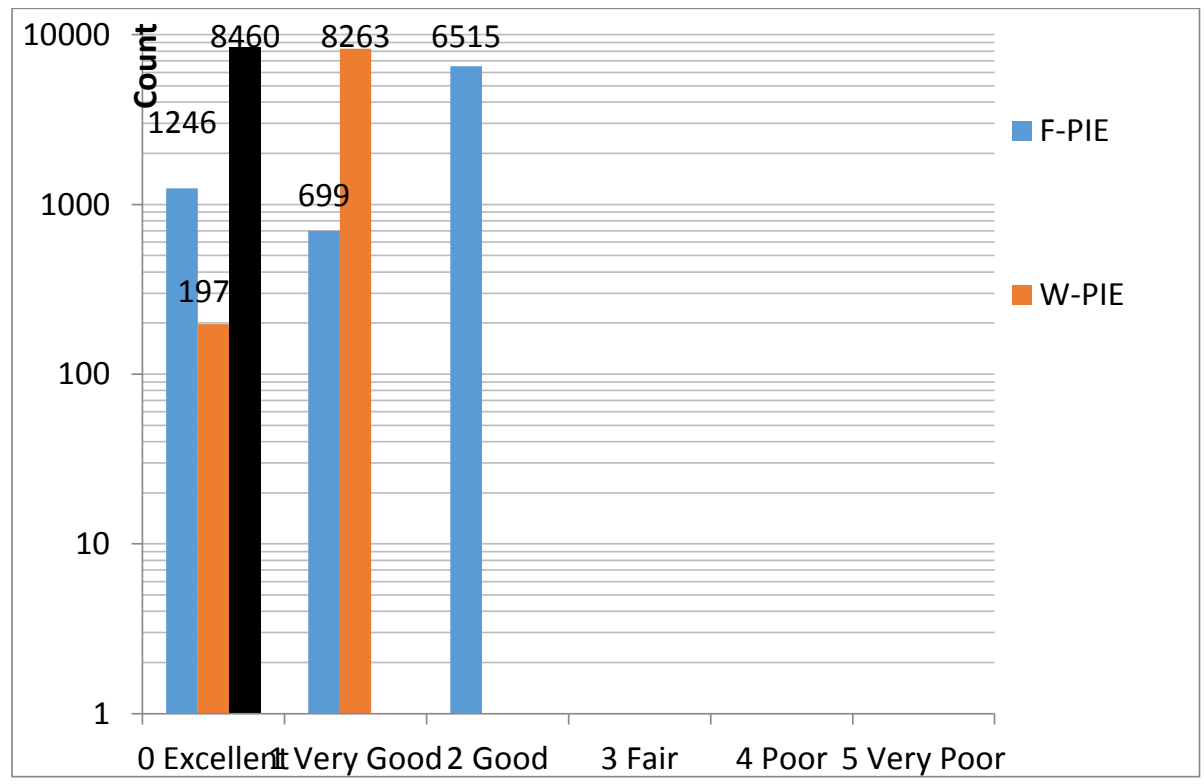

Figure G-10. Performance Index by W-PIE and F-PIE. 
The results from both W-PIE and F-PIE show that all of the pipes are 0,1 , and 2 . There are no Fair (3), poor (4), and very poor (5) pipes in this evaluation because of the lack of key parameters such as pipe condition. The results from F-PIE and W-PIE show more very good and good pipes indicating that the vales are shifted from excellent because of the knowledge base.

\subsection{Result Comparisons}

The results calculated by the weighted factor and fuzzy inference models are compared to PACP ratings in Tables G-21, and G-22. Table G-23 compares the results between W-PIE and FPIE.

Table G-21. PACP and F-PIE Comparison.

\begin{tabular}{|c|c|c|c|c|c|c|}
\hline & & & \multicolumn{3}{|c|}{ F_PIE } & \multirow[b]{2}{*}{ Total } \\
\hline & & & 0 & 1 & 2 & \\
\hline \multirow[t]{4}{*}{ PACP } & 0 & No. of pipes & 1246 & 699 & 6515 & 8460 \\
\hline & & $\%$ within $\mathrm{PACP}^{*}$ & $14.7 \%$ & $8.3 \%$ & $77.0 \%$ & $100.0 \%$ \\
\hline & & $\%$ within $\mathrm{F}_{-} \mathrm{PIE} \mathrm{E}^{\star *}$ & $100.0 \%$ & $100.0 \%$ & $100.0 \%$ & $100.0 \%$ \\
\hline & & $\%$ of Total ${ }^{\star \star *}$ & $14.7 \%$ & $8.3 \%$ & $77.0 \%$ & $100.0 \%$ \\
\hline \multirow{4}{*}{\multicolumn{2}{|c|}{ Total }} & No. of pipes & 1246 & 699 & 6515 & 8460 \\
\hline & & $\%$ within PACP & $14.7 \%$ & $8.3 \%$ & $77.0 \%$ & $100.0 \%$ \\
\hline & & $\%$ within F_PIE & $100.0 \%$ & $100.0 \%$ & $100.0 \%$ & $100.0 \%$ \\
\hline & & $\%$ of Total & $14.7 \%$ & $8.3 \%$ & $77.0 \%$ & $100.0 \%$ \\
\hline
\end{tabular}

* \% within PACP $=\frac{\text { Number of pipes }}{\text { Row Total }(P A C P)} \times 100$

** \% within $F-P I E=\frac{\text { Number of pipes }}{\text { Column Total }(F-P I E)} \times 100$

$* * * \%$ of Total $=\frac{\text { Number of pipes }}{\text { Total }} \times 100$ 
Table G-22. PACP and W-PIE Comparison.

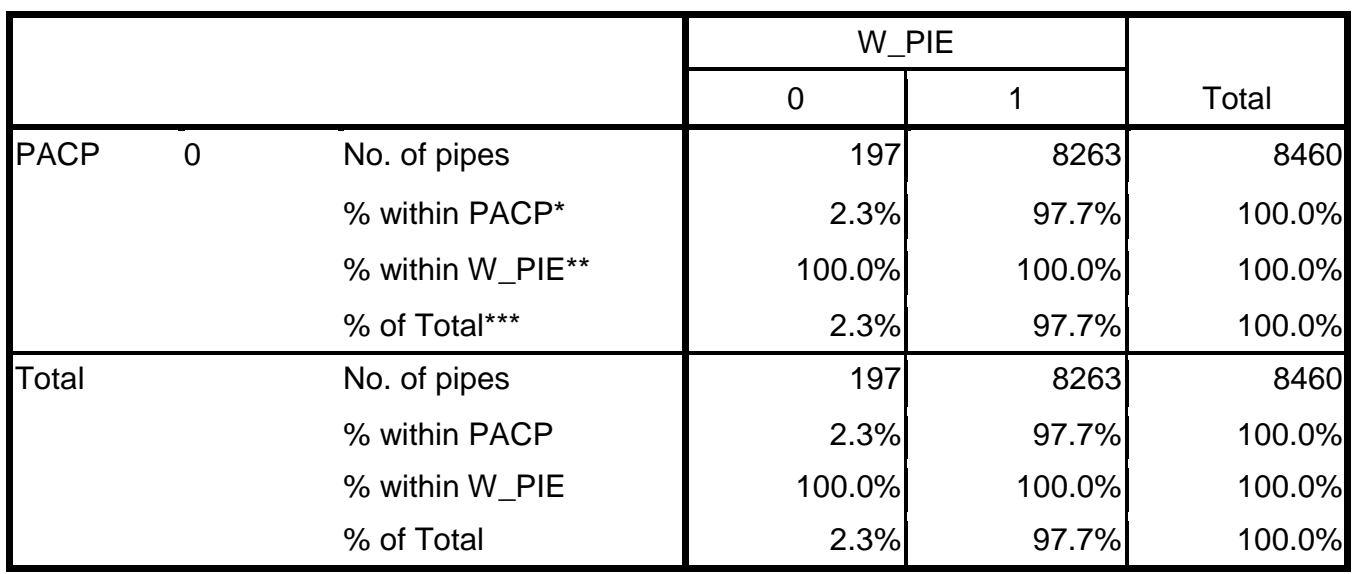

$* \%$ within PACP $=\frac{\text { Number of pipes }}{\text { Row Total }(P A C P)} \times 100$

** \% within $W-P I E=\frac{\text { Number of pipes }}{\text { Column Total }(F-P I E)} \times 100$

*** \% of Total $=\frac{\text { Number of pipes }}{\text { Total }} \times 100$ 
Table G-23. W-PIE and F-PIE Comparison.

\begin{tabular}{|c|c|c|c|c|c|c|}
\hline & \multicolumn{3}{|c|}{ F-PIE } & \multirow[b]{2}{*}{ Total } \\
\hline & & & 0 & 1 & 2 & \\
\hline \multirow[t]{8}{*}{ WF } & 0 & No. of pipes & 716 & 403 & 3738 & 4857 \\
\hline & & $\%$ within W-PIE & $14.7 \%$ & $8.3 \%$ & $77.0 \%$ & $100.0 \%$ \\
\hline & & $\%$ within F-PIE ** & $57.5 \%$ & $57.7 \%$ & $57.4 \%$ & $57.4 \%$ \\
\hline & & $\%$ of Total ${ }^{\star \star \star}$ & $8.5 \%$ & $4.8 \%$ & $44.2 \%$ & $57.4 \%$ \\
\hline & 1 & No. of pipes & 530 & 296 & 2777 & 3603 \\
\hline & & $\%$ within W-PIE & $14.7 \%$ & $8.2 \%$ & $77.1 \%$ & $100.0 \%$ \\
\hline & & $\%$ within F-PIE & $42.5 \%$ & $42.3 \%$ & $42.6 \%$ & $42.6 \%$ \\
\hline & & $\%$ of Total & $6.3 \%$ & $3.5 \%$ & $32.8 \%$ & $42.6 \%$ \\
\hline \multirow[t]{4}{*}{ Total } & & No. of pipes & 1246 & 699 & 6515 & 8460 \\
\hline & & $\%$ within W-PIE & $14.7 \%$ & $8.3 \%$ & $77.0 \%$ & $100.0 \%$ \\
\hline & & $\%$ within F-PIE & $100.0 \%$ & $100.0 \%$ & $100.0 \%$ & $100.0 \%$ \\
\hline & & $\%$ of Total & $14.7 \%$ & $8.3 \%$ & $77.0 \%$ & $100.0 \%$ \\
\hline
\end{tabular}

$* \%$ within $W-P I E=\frac{\text { Number of pipes }}{\text { Row } \operatorname{Total}(W-P I E)} \times 100$

** \% within $F-P I E=\frac{\text { Number of pipes }}{\text { Column } \operatorname{Total}(F-P I E)} \times 100$

$* * * \%$ of Total $=\frac{\text { Number of pipes }}{\text { Total }} \times 100$

\subsection{Sample Calculations}

Performance Index has been calculated for various pipe sections (manhole to manhole). These sample pipes are from the City I's data. This exercise was conducted to understand the benefits and shortcomings of the performance model. For demonstration of the performance index, some parameters were utility data however; others were derived and may be inaccurate. 


\section{Sample 1: Pipe ID 50}

Pipe ID 50 was selected for sample calculations. Pipe details are given in Table G-24.

Table G-24. Sample 1 Pipe Data.

\begin{tabular}{|l|l|l|l|}
\hline No. & Parameter & Unit & Value \\
\hline 1 & Pipe Diameter & Inch & 8 \\
\hline 2 & Pipe Length & Feet & 357 \\
\hline
\end{tabular}

* Parameters indicated in red are unfavorable parameters

\section{PACP Rating Index:}

The value of the pipe rating by PACP is " 0 no data". There are no inspection data of this pipe.

\section{W-PIE Index:}

The Performance Index of this pipe had a value of "1 (very good)".

\section{F-PIE Index:}

The Performance Index of this pipe had a value of " 2 (good)".

\section{Index Comparisons: No data \& Average}

PACP rating index of this pipe 0 indicates that the pipe is in an Excellent condition with the value of " 0 no inspection data". The performance index gives value ' 2 ' indicating good performance, There may be a deposit or grease build up due to small diameter and length of greater than $300 \mathrm{ft}$ which is a regular cleaning equipment length. This problem can cause blockage if regular cleaning has not been performed. 


\section{City J}

We analyzed data received from City $\mathrm{J}$ and evaluated each pipe section (manhole to manhole) using performance models (weighted factor and fuzzy inference system). There was 1 geo-database table which consists mainly of wastewater pipe data. There were five parameters used in this study.

\section{Parameters}
a. Pipe age
b. Pipe diameter
c. Pipe slope
d. Pipe length
e. Pipe material

The parameters listed above are stored as attributes in mainline table. The performance models were used to evaluate received data with the results show below.

\subsection{W-PIE and F-PIE Results}

Figure G-11 is a bar chart of the performance index calculated for no PACP data, W-PIE and F-PIE. The $\mathrm{x}$-axis is the performance index and y-axis is the number of pipes that fall within each value. The pipes that do not have inspection data, indicated in black, are assumed excellent for the purpose of calculation.

The results from both W-PIE and F-PIE show that all of the pipes are 0, 1, 2 and 3 which are in excellent, very good, good, and fair respectively. There are no poor (4) or very poor (5) rated pipes in this evaluation because of the lack of key parameters such as pipe condition. The main parameter that influences the ratings is pipe slope. The results from F-PIE and W-PIE show more very good and good pipes. These ratings infer that these pipes are shifted from excellent pipes because of the knowledge base. 


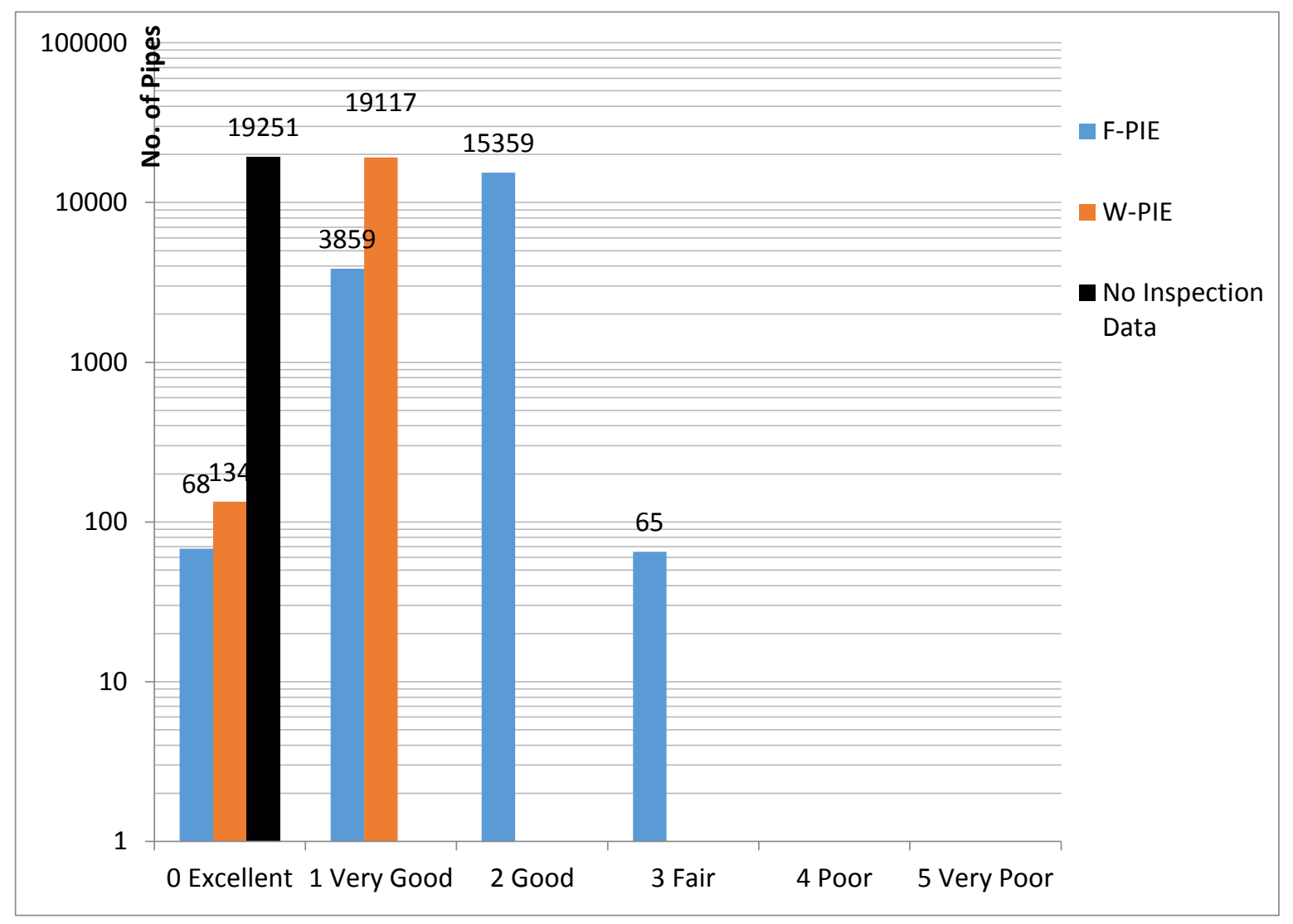

Figure G-11. Performance Index by No PACP data, F-PIE and W-PIE

\subsection{Result Comparisons}

The results calculated by weighted factor and fuzzy inference models are compared to PACP ratings in Tables G-25, and G-26. Table G-27 shows the results obtained by F-PIE compared to the PACP rating index. The PACP rating index is in rows, and the results from FPIE are in columns. Table G-26 shows the performance index calculated from weighted factors compared to the PACP rating index and Table G-27 shows the comparison of the results between weighted factor and fuzzy inference models. The tables provide similar information as a bar chart above but in more detail. 
Table G-25. PACP and F-PIE Comparison.

\begin{tabular}{|c|c|c|c|c|c|c|}
\hline & \multicolumn{4}{|c|}{ F_PIE } & \multirow[b]{2}{*}{ Total } \\
\hline & & 0 & 1 & 2 & 3 & \\
\hline \multirow{4}{*}{$\begin{array}{c}\text { PACP } 0 \text { (No PACP } \\
\text { data) }\end{array}$} & No. of pipes & 1 & 3636 & 23501 & 1690 & 28828 \\
\hline & $\%$ within $\mathrm{PACP}^{*}$ & $.0 \%$ & $12.6 \%$ & $81.5 \%$ & $5.9 \%$ & $100.0 \%$ \\
\hline & $\begin{array}{l}\% \text { within } \\
\text { F_PIE }\end{array}$ & $100.0 \%$ & $100.0 \%$ & $100.0 \%$ & $100.0 \%$ & $100.0 \%$ \\
\hline & $\%$ of Total ${ }^{\star \star \star}$ & $.0 \%$ & $12.6 \%$ & $81.5 \%$ & $5.9 \%$ & $100.0 \%$ \\
\hline \multirow[t]{4}{*}{ Total } & No. of pipes & 1 & 3636 & 23501 & 1690 & 28828 \\
\hline & $\%$ within PACP & $.0 \%$ & $12.6 \%$ & $81.5 \%$ & $5.9 \%$ & $100.0 \%$ \\
\hline & $\%$ within F_PIE & $100.0 \%$ & $100.0 \%$ & $100.0 \%$ & $100.0 \%$ & $100.0 \%$ \\
\hline & $\%$ of Total & $.0 \%$ & $12.6 \%$ & $81.5 \%$ & $5.9 \%$ & $100.0 \%$ \\
\hline
\end{tabular}

* \% within PACP $=\frac{\text { Number of pipes }}{\text { Row } \text { Total }(P A C P)} \times 100$

** \% within $F-P I E=\frac{\text { Number of pipes }}{\text { Column Total }(F-P I E)} \times 100$

$* * * \%$ of Total $=\frac{\text { Number of pipes }}{\text { Total }} \times 100$

Table G-26. PACP and W-PIE Comparison.

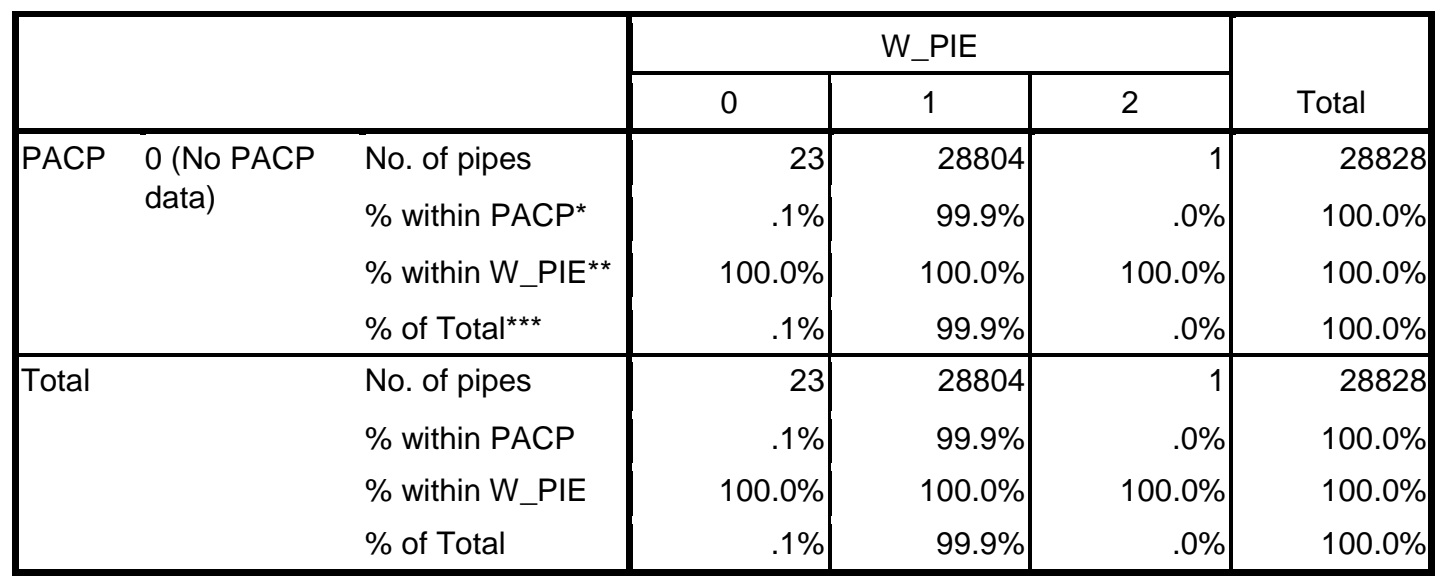

*\% within PACP $=\frac{\text { Number of pipes }}{\text { Row } \text { Total }(P A C P)} \times 100$

$* * \%$ within $W-P I E=\frac{\text { Number of pipes }}{\text { Column Total }(W-P I E)} \times 100$

$* * * \%$ of Total $=\frac{\text { Number of pipes }}{\text { Total }} \times 100$ 
Table G-27. W-PIE and F-PIE Comparison.

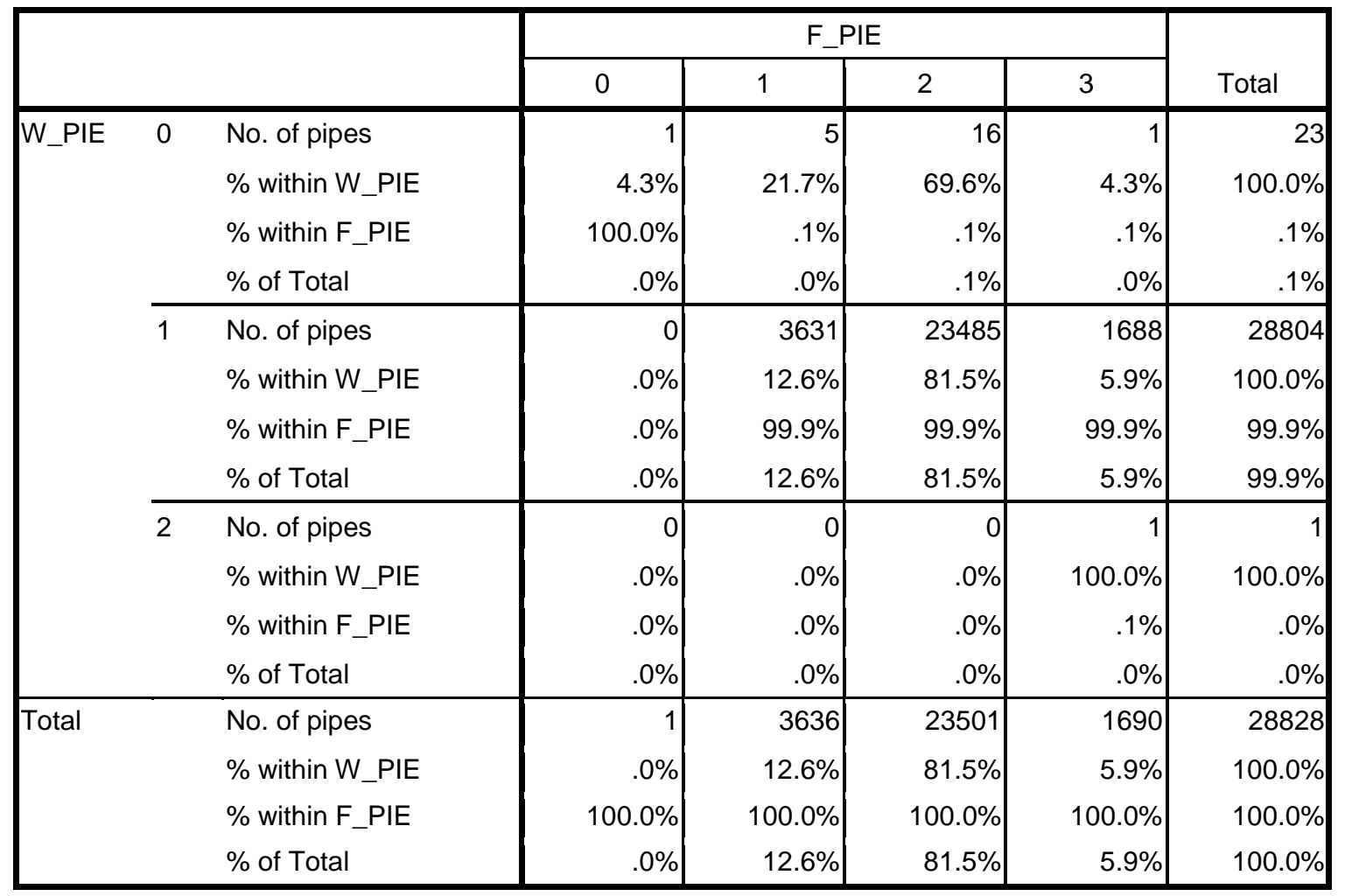

$* \%$ within $W-P I E=\frac{\text { Number of pipes }}{\text { Row } \operatorname{Total}(W-P I E)} \times 100$

** \% within $F-P I E=\frac{\text { Number of pipes }}{\text { Column Total }(F-P I E)} \times 100$

$* * * \%$ of Total $=\frac{\text { Number of pipes }}{\text { Total }} \times 100$ 


\section{City K}

We analyzed data received from City $\mathrm{K}$ and evaluated each pipe section (manhole to manhole) using performance models (W-PIE and F-PIE). Altogether, there were several main geo-database tables, which consist of sewer gravity, force mains, and manholes. Five parameters can be used in this pilot study.

\section{Parameters}
a. Pipe material
b. Pipe diameter
c. Pipe age
d. Pipe slope
e. Pipe length

The parameters listed above are stored as attributes in mainline tables. Based on limited data, the performance models produced the results presented below.

\subsection{W-PIE and F-PIE Results}

Figure G-12 shows a bar chart of the performance index calculated by W-PIE and F-PIE. The $\mathrm{x}$-axis is the performance index and $\mathrm{y}$-axis is the number of pipes that fall within each value. The pipes that do not have inspection data, indicated in black, are assumed excellent for the purpose of calculation.

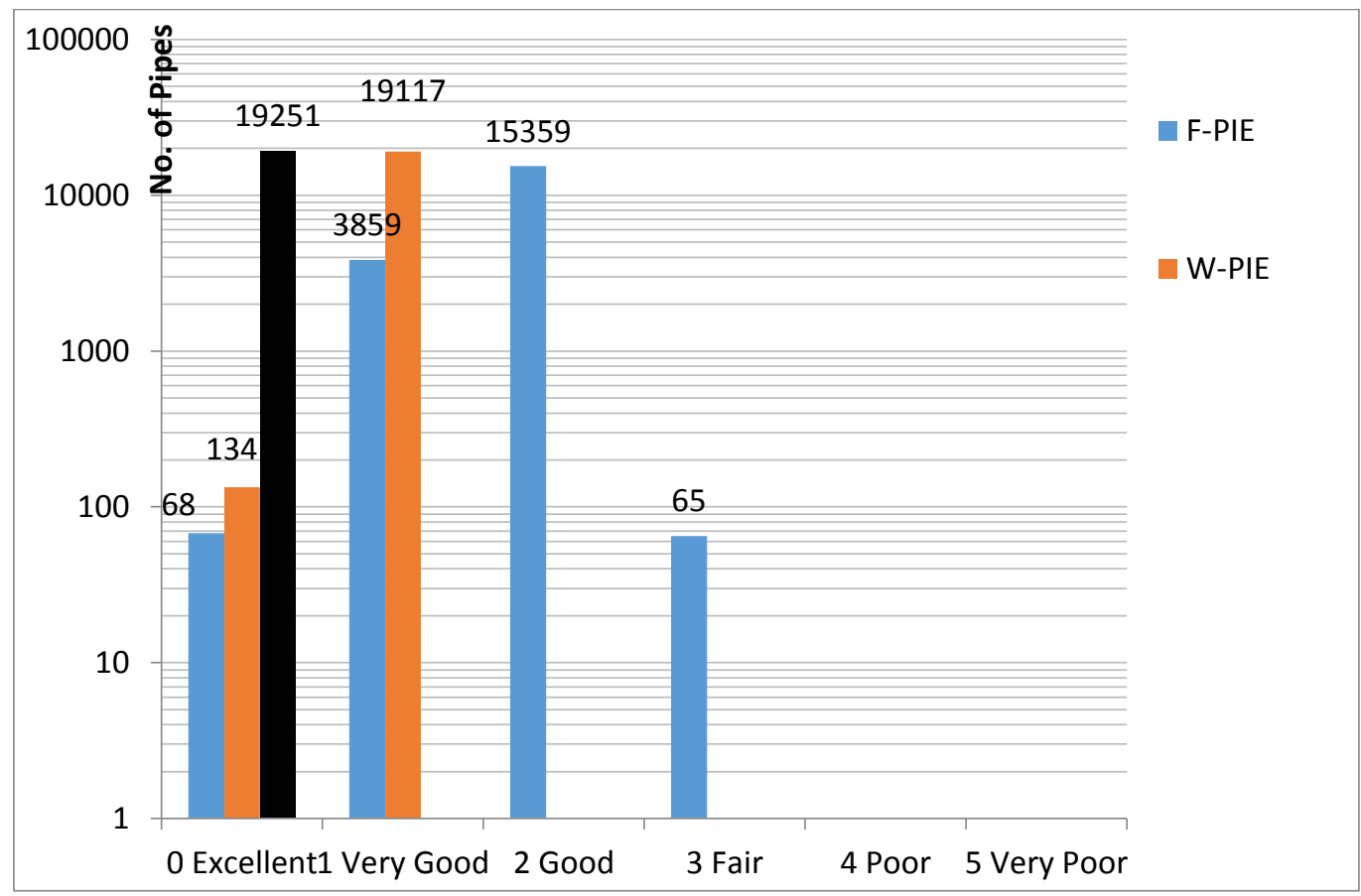

Figure G-12. Performance Index by W-PIE and F-PIE. 
The results from F-PIE show pipes are $0,1,2$, and 3 which are excellent, very good, good, and fair respectively. The results from W-PIE show pipes are 0 , and 1 which are in excellent, and very good respectively.

There are no poor (4), or very poor (5) pipes in this evaluation because of the lack of key parameters such as pipe condition. The results from F-PIE and W-PIE show more very good and good pipes. These ratings infer that these pipes are shifted from excellent pipes because of the knowledge base.

\subsection{Result Comparisons}

The results calculated by weighted factor and fuzzy inference models are compared to PACP ratings in Tables G-28, and G-29. Table G-30 shows the comparison of the results between W-PIE and F-PIE.

Table G-28. PACP and F-PIE Comparison.

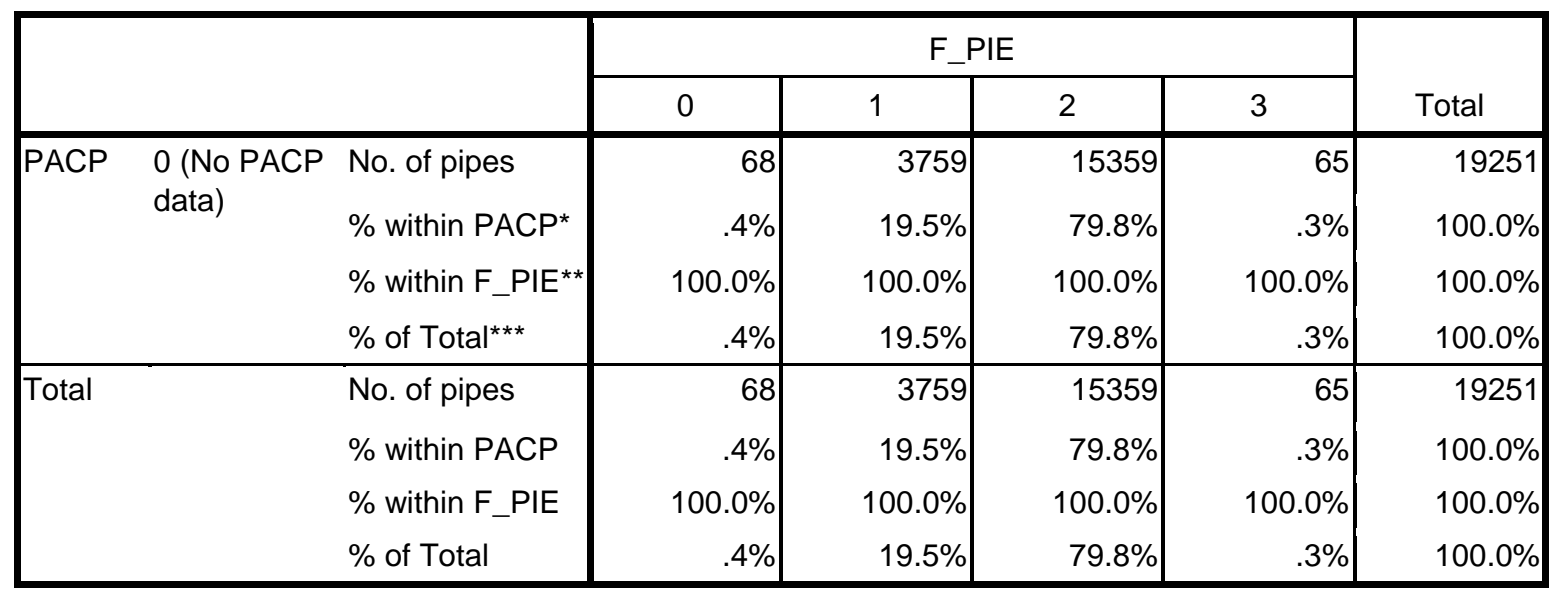

* \% within PACP $=\frac{\text { Number of pipes }}{\text { Row } \text { Total }(P A C P)} \times 100$

** \% within F - PIE $=\frac{\text { Number of pipes }}{\text { Column Total }(F-P I E)} \times 100$

$* * * \%$ of Total $=\frac{\text { Number of pipes }}{\text { Total }} \times 100$ 
Table G-29. PACP and W-PIE Comparison.

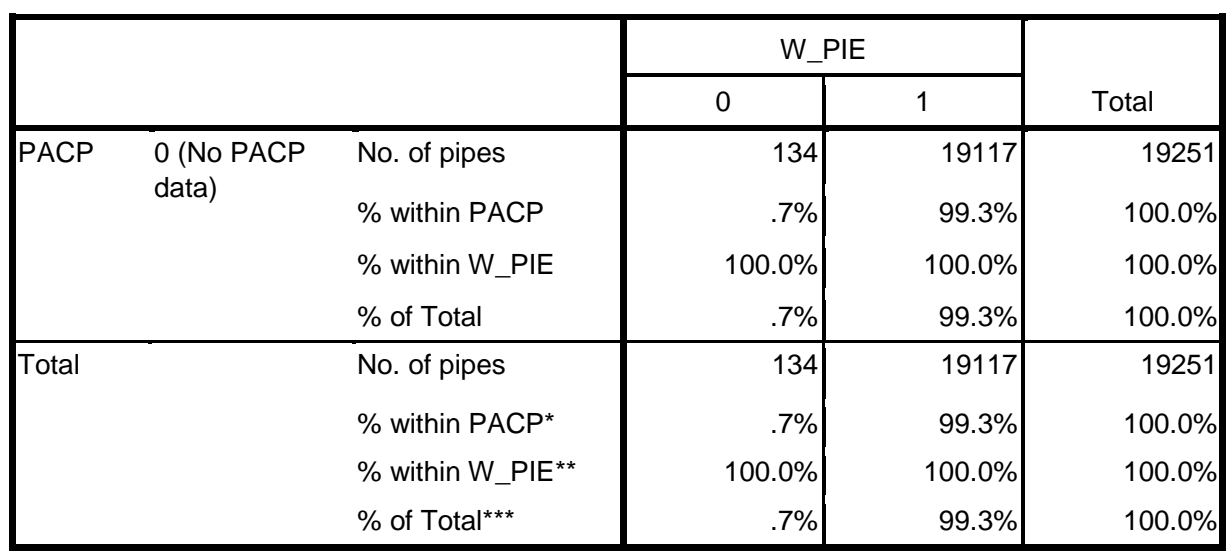

*\% within PACP $=\frac{\text { Number of pipes }}{\text { Row } \text { Total }(P A C P)} \times 100$

** \% within $W-P I E=\frac{\text { Number of pipes }}{\text { Column Total }(W-P I E)} \times 100$

$* * * \%$ of Total $=\frac{\text { Number of pipes }}{\text { Total }} \times 100$

Table G-30. W-PIE and F-PIE Comparison.

\begin{tabular}{|c|c|c|c|c|c|c|c|}
\hline & & & \multicolumn{4}{|c|}{ F_PIE } & \multirow[b]{2}{*}{ Total } \\
\hline & & & 0 & 1 & 2 & 3 & \\
\hline \multirow[t]{8}{*}{ W_PIE } & 0 & No. of pipes & 47 & 73 & 14 & 0 & 134 \\
\hline & & \% within W_PIE* & $35.1 \%$ & $54.5 \%$ & $10.4 \%$ & $.0 \%$ & $100.0 \%$ \\
\hline & & $\%$ within $\mathrm{F} \_\mathrm{PIE}{ }^{* *}$ & $69.1 \%$ & $1.9 \%$ & $.1 \%$ & $.0 \%$ & $.7 \%$ \\
\hline & & $\%$ of Total ${ }^{\star \star *}$ & $.2 \%$ & $.4 \%$ & $.1 \%$ & $.0 \%$ & $.7 \%$ \\
\hline & 1 & No. of pipes & 21 & 3686 & 15345 & 65 & 19117 \\
\hline & & \% within W_PIE & $.1 \%$ & $19.3 \%$ & $80.3 \%$ & $.3 \%$ & $100.0 \%$ \\
\hline & & $\%$ within $F_{-}$PIE & $30.9 \%$ & $98.1 \%$ & $99.9 \%$ & $100.0 \%$ & $99.3 \%$ \\
\hline & & $\%$ of Total & $.1 \%$ & $19.1 \%$ & $79.7 \%$ & $.3 \%$ & $99.3 \%$ \\
\hline \multirow[t]{4}{*}{ Total } & & No. of pipes & 68 & 3759 & 15359 & 65 & 19251 \\
\hline & & \% within W_PIE & $.4 \%$ & $19.5 \%$ & $79.8 \%$ & $.3 \%$ & $100.0 \%$ \\
\hline & & $\%$ within $\mathrm{F} \_\mathrm{PIE}$ & $100.0 \%$ & $100.0 \%$ & $100.0 \%$ & $100.0 \%$ & $100.0 \%$ \\
\hline & & $\%$ of Total & $.4 \%$ & $19.5 \%$ & $79.8 \%$ & $.3 \%$ & $100.0 \%$ \\
\hline
\end{tabular}

$* \%$ within $W-P I E=\frac{\text { Number of pipes }}{\text { Row } \operatorname{Total}(W-P I E)} \times 100$

** $\%$ within $F-P I E=\frac{\text { Number of pipes }}{\text { Column Total }(F-P I E)} \times 100$

$* * * \%$ of Total $=\frac{\text { Number of pipes }}{\text { Total }} \times 100$ 


\section{APPENDIX H}

\section{EXAMPLES OF QUESTIONNAIRE RESPONSES}




\section{Performance Index for Wastewater Pipes}

\section{Please rate the importance of each parameter according to your judgment.}

\section{Response from WVWA.}

\section{a. Structural Index}

\begin{tabular}{|c|c|c|c|c|}
\hline Parameters & $\begin{array}{c}\text { Significance } \\
\qquad(1-5) \\
5=\text { very high } \\
\text { For Concrete } \\
\text { pipe }\end{array}$ & $\begin{array}{c}\text { Significance } \\
\qquad(1-5) \\
5=\text { very high } \\
\text { For Clay pipe }\end{array}$ & $\begin{array}{c}\text { Significance } \\
\qquad(1-5) \\
5 \text { =very high } \\
\text { For Metal pipe }\end{array}$ & $\begin{array}{c}\text { Significance } \\
\qquad(1-5) \\
5 \text { =very high } \\
\text { For Plastic pipe }\end{array}$ \\
\hline \multicolumn{5}{|l|}{ 1. External Corrosion Module } \\
\hline Groundwater Table & 4 & 2 & 4 & - \\
\hline Pipe Age & 4 & 2 & 4 & - \\
\hline Pipe Depth & 2 & 2 & 2 & - \\
\hline Soil Resistivity & 5 & 1 & 5 & - \\
\hline Pipe Wall Thickness & 4 & 2 & 4 & - \\
\hline Soil pH & - & - & - & - \\
\hline Soil Sulfate & - & - & - & - \\
\hline Soil Chloride & - & - & - & - \\
\hline Redox Potential & - & - & - & - \\
\hline Stray Currents & 4 & 2 & 5 & - \\
\hline \multicolumn{5}{|l|}{ 2. Internal Corrosion Module } \\
\hline Pipe Slope & 3 & 1 & 3 & - \\
\hline Pipe Age & 3 & 2 & 4 & - \\
\hline Flow Velocity & 3 & 1 & 3 & - \\
\hline Flow depth/Diameter (d/D) & 4 & 2 & 4 & - \\
\hline Wastewater $\mathrm{pH}$ & - & - & - & - \\
\hline Wastewater Sulfate & - & - & - & - \\
\hline $\mathrm{H} 2 \mathrm{~S}$ & 5 & 1 & 5 & - \\
\hline Maintenance Frequency & 4 & 2 & 4 & - \\
\hline \multicolumn{5}{|l|}{ 3. Surface Wear Module } \\
\hline Pipe Age & 4 & 2 & 3 & 1 \\
\hline Flow Velocity & 4 & 2 & 2 & 1 \\
\hline
\end{tabular}




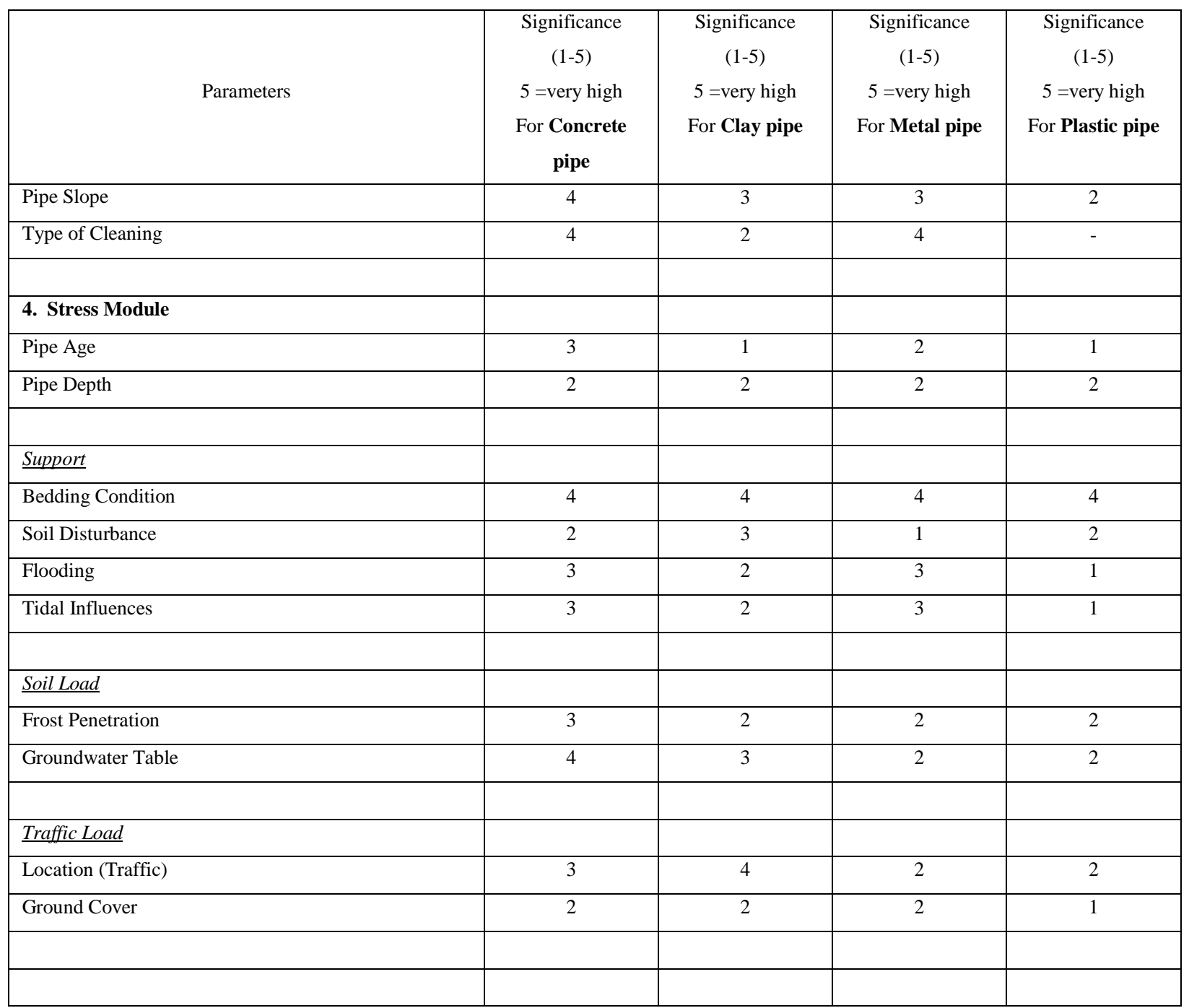




\section{b. Functional Index}

\begin{tabular}{|c|c|c|c|c|}
\hline Parameters & $\begin{array}{c}\text { Significance } \\
\qquad(1-5) \\
5=\text { very high } \\
\text { For Concrete } \\
\text { pipe }\end{array}$ & $\begin{array}{l}\text { Significance } \\
\qquad(1-5) \\
5=\text { very high } \\
\text { For Clay pipe }\end{array}$ & $\begin{array}{c}\text { Significance } \\
\qquad(1-5) \\
5 \text { =very high } \\
\text { For Metal pipe }\end{array}$ & $\begin{array}{c}\text { Significance } \\
\qquad(1-5) \\
5=\text { very high } \\
\text { For Plastic pipe }\end{array}$ \\
\hline \multicolumn{5}{|l|}{ 1. Infiltration /Exfiltration Module } \\
\hline Pipe Age & - & - & - & - \\
\hline Groundwater Table & 5 & 5 & 3 & 2 \\
\hline Soil Type & 4 & 4 & 2 & 2 \\
\hline Pipe Surcharging & 4 & 5 & 2 & 2 \\
\hline \multicolumn{5}{|l|}{ 2. Blockage Module } \\
\hline Pipe Age & 1 & 1 & 1 & 1 \\
\hline Pipe Length & 2 & 2 & 2 & 2 \\
\hline Wall thickness & 1 & 1 & 1 & 1 \\
\hline Pipe Diameter & 3 & 3 & 3 & 3 \\
\hline Pipe Slope & 3 & 3 & 3 & 3 \\
\hline Flow Velocity & 3 & 3 & 3 & 3 \\
\hline Flow depth/Diameter (d/D) & 3 & 3 & 3 & 3 \\
\hline Density of Connection & 3 & 3 & 3 & 3 \\
\hline Maintenance Frequency & 4 & 4 & 4 & 4 \\
\hline \multicolumn{5}{|l|}{ 3. Root Penetration Module } \\
\hline Pipe Age & 3 & 3 & 2 & 2 \\
\hline Pipe Wall Thickness & 3 & 2 & 2 & 2 \\
\hline Pipe Diameter & 4 & 4 & 3 & 3 \\
\hline Proximity to trees & 5 & 5 & 5 & 5 \\
\hline Maintenance Frequency & 4 & 4 & 4 & 4 \\
\hline \multicolumn{5}{|l|}{ 4. Hydraulic (Capacity) Module } \\
\hline Flow depth/Diameter (d/D) & 4 & 4 & 4 & 4 \\
\hline Flow Velocity & 3 & 3 & 3 & 3 \\
\hline Pipe Slope & 4 & 4 & 4 & 4 \\
\hline Pipe Surcharging & 5 & 5 & 5 & 5 \\
\hline Maintenance Frequency & 5 & 5 & 5 & 5 \\
\hline
\end{tabular}




\section{Performance Index for Wastewater Pipes}

\section{Please rate the importance of each parameter according to your judgment.}

\section{Response from OCSD.}

\section{a. Structural Index}

\begin{tabular}{|c|c|c|c|c|}
\hline Parameters & $\begin{array}{c}\text { Significance } \\
\qquad(1-5) \\
5 \text { =very high } \\
\text { For Concrete } \\
\text { pipe }\end{array}$ & $\begin{array}{l}\text { Significance } \\
\qquad(1-5) \\
5 \text { =very high } \\
\text { For Clay pipe }\end{array}$ & $\begin{array}{l}\text { Significance } \\
\qquad(1-5) \\
5=\text { very high } \\
\text { For Metal pipe }\end{array}$ & $\begin{array}{c}\text { Significance } \\
\qquad(1-5) \\
5 \text { =very high } \\
\text { For Plastic pipe }\end{array}$ \\
\hline \multicolumn{5}{|l|}{ 1. External Corrosion Module } \\
\hline Groundwater Table & - & - & - & - \\
\hline Pipe Age & 5 & 1 & 5 & 1 \\
\hline Pipe Depth & - & 1 & - & 1 \\
\hline Soil Resistivity & 2 & 1 & 5 & 1 \\
\hline Pipe Wall Thickness & 5 & 1 & 5 & 1 \\
\hline Soil pH & 3 & 1 & 5 & 1 \\
\hline Soil Sulfate & 5 & 1 & 3 & 1 \\
\hline Soil Chloride & 3 & 1 & 5 & 1 \\
\hline Redox Potential & 2 & 1 & 5 & 1 \\
\hline Stray Currents & 1 & 1 & 5 & 1 \\
\hline \multicolumn{5}{|l|}{ 2. Internal Corrosion Module } \\
\hline Pipe Slope & 5 & 1 & 5 & 1 \\
\hline Pipe Age & 5 & 1 & 5 & 1 \\
\hline Flow Velocity & 5 & 1 & 5 & 1 \\
\hline Flow depth/Diameter (d/D) & 5 & 1 & 5 & 1 \\
\hline Wastewater $\mathrm{pH}$ & 3 & 1 & 3 & 1 \\
\hline Wastewater Sulfate & 5 & 1 & 3 & 1 \\
\hline $\mathrm{H} 2 \mathrm{~S}$ & 5 & 1 & 5 & 1 \\
\hline Maintenance Frequency & - & - & - & - \\
\hline \multicolumn{5}{|l|}{ 3. Surface Wear Module } \\
\hline Pipe Age & 4 & 3 & 5 & 2 \\
\hline
\end{tabular}




\begin{tabular}{|c|c|c|c|c|}
\hline Parameters & $\begin{array}{c}\text { Significance } \\
\qquad(1-5) \\
5=\text { very high } \\
\text { For Concrete } \\
\text { pipe }\end{array}$ & $\begin{array}{c}\text { Significance } \\
\qquad(1-5) \\
5=\text { very high } \\
\text { For Clay pipe }\end{array}$ & $\begin{array}{c}\text { Significance } \\
\qquad(1-5) \\
5 \text { =very high } \\
\text { For Metal pipe }\end{array}$ & $\begin{array}{c}\text { Significance } \\
\qquad(1-5) \\
5=\text { very high } \\
\text { For Plastic pipe }\end{array}$ \\
\hline Flow Velocity & 4 & 3 & 5 & 2 \\
\hline Pipe Slope & 4 & 3 & 5 & 2 \\
\hline Type of Cleaning & - & - & - & - \\
\hline \multicolumn{5}{|l|}{ 4. Stress Module } \\
\hline Pipe Age & 5 & 5 & 5 & 5 \\
\hline Pipe Depth & 5 & 5 & 5 & 5 \\
\hline \multicolumn{5}{|l|}{ Support } \\
\hline Bedding Condition & 3 & 5 & 4 & 5 \\
\hline Soil Disturbance & - & - & - & - \\
\hline Flooding & 1 & 3 & 3 & 5 \\
\hline Tidal Influences & 1 & 1 & 1 & 1 \\
\hline \multicolumn{5}{|l|}{$\underline{\text { Soil Load }}$} \\
\hline Frost Penetration & - & - & - & - \\
\hline Groundwater Table & 1 & 5 & 3 & 1 \\
\hline \multicolumn{5}{|l|}{ Traffic Load } \\
\hline Location (Traffic) & 1 & 5 & 3 & 3 \\
\hline Ground Cover & 3 & 5 & 4 & 4 \\
\hline & & & & \\
\hline
\end{tabular}




\section{b. Functional Index}

\begin{tabular}{|c|c|c|c|c|}
\hline Parameters & $\begin{array}{c}\text { Significance } \\
\qquad(1-5) \\
5=\text { very high } \\
\text { For Concrete } \\
\text { pipe }\end{array}$ & $\begin{array}{l}\text { Significance } \\
\qquad(1-5) \\
5 \text { =very high } \\
\text { For Clay pipe }\end{array}$ & $\begin{array}{c}\text { Significance } \\
\qquad(1-5) \\
5=\text { very high } \\
\text { For Metal pipe }\end{array}$ & $\begin{array}{c}\text { Significance } \\
\qquad(1-5) \\
5 \text { =very high } \\
\text { For Plastic pipe }\end{array}$ \\
\hline \multicolumn{5}{|l|}{ 1. Infiltration /Exfiltration Module } \\
\hline Pipe Age & - & - & - & - \\
\hline Groundwater Table & 1 & 5 & 3 & 1 \\
\hline Soil Type & 3 & 5 & 2 & 1 \\
\hline Pipe Surcharging & 3 & 5 & 2 & 1 \\
\hline \multicolumn{5}{|l|}{ 2. Blockage Module } \\
\hline Pipe Age & 2 & 2 & 2 & 2 \\
\hline Pipe Length & 2 & 3 & 1 & 1 \\
\hline Wall thickness & 4 & 5 & 2 & 1 \\
\hline Pipe Diameter & 5 & 5 & 5 & 5 \\
\hline Pipe Slope & 5 & 5 & 5 & 5 \\
\hline Flow Velocity & - & - & - & - \\
\hline Flow depth/Diameter (d/D) & - & - & - & - \\
\hline Density of Connection & 3 & 5 & 1 & 1 \\
\hline Maintenance Frequency & - & - & - & - \\
\hline \multicolumn{5}{|l|}{ 3. Root Penetration Module } \\
\hline Pipe Age & 3 & 5 & 2 & 1 \\
\hline Pipe Wall Thickness & 1 & - & - & - \\
\hline Pipe Diameter & - & - & - & - \\
\hline Proximity to trees & - & - & - & - \\
\hline Maintenance Frequency & - & - & - & - \\
\hline \multicolumn{5}{|l|}{ 4. Hydraulic (Capacity) Module } \\
\hline Flow depth/Diameter (d/D) & 2 & 2 & 1 & 1 \\
\hline Flow Velocity & - & - & - & - \\
\hline Pipe Slope & - & - & - & - \\
\hline Pipe Surcharging & 3 & 5 & 2 & 1 \\
\hline Maintenance Frequency & - & - & - & - \\
\hline & & & & \\
\hline
\end{tabular}

